\title{
Sound absorption of
}

\section{porous structures}

a design tool for road surfaces 



\section{Sound absorption of porous structures}

a design tool for road surfaces

M. Bezemer-Krijnen 
Faculty of Engineering Technology, University of Twente
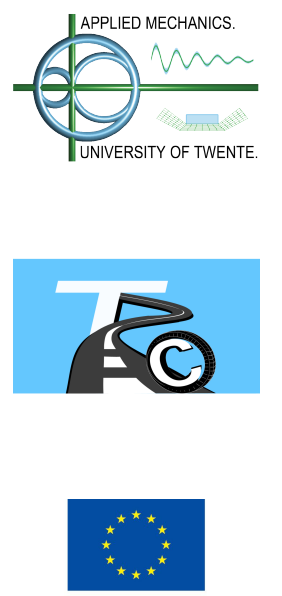

Europese Unie Europees Fonds voor Regionale Ontwikkeling

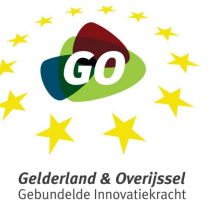

Applied Mechanics - Structural Dynamics, Acoustics \& Control (SDAC)

Tire-Road Consortium

This research has been conducted as part of the project 'Stil Veilig Wegverkeer', funded by $\mathrm{Eu}$ ropees Fonds voor Regionale Ontwikkeling.

This research has been conducted as part of the project 'Stil Veilig Wegverkeer', funded by $G O$ Gebundelde Innovatiekracht.

Title: $\quad$ Sound absorption of porous structures a design tool for road surfaces

Author: M. Bezemer-Krijnen

ISBN: $\quad$ 978-90-365-4584-6

DOI: $\quad 10.3990 / 1.9789036545846$

Cover design by Marieke Bezemer-Krijnen.

Copyright (c) 2018 by M. Bezemer-Krijnen, Enschede, The Netherlands.

All rights reserved. No part of this publication may be reproduced by print, photocopy or any other means without the prior written permission from the copyright owner.

Printed by Gildeprint - www.gildeprint.nl 


\title{
SOUND ABSORPTION OF POROUS STRUCTURES
}

\author{
A DESIGN TOOL FOR ROAD SURFACES
}

\author{
DISSERTATION \\ to obtain \\ the degree of doctor at the University of Twente, \\ on the authority of the rector magnificus, \\ prof. dr. T.T.M. Palstra, \\ on account of the decision of the graduation committee, \\ to be publicly defended \\ on Friday the 20th of July 2018 at 16.45 hours
}

by

Marieke Bezemer-Krijnen
born on the 16th of June 1985
in Apeldoorn, The Netherlands 
This dissertation has been approved by:

Supervisor: prof. dr. ir. A. de Boer

Co-supervisor: dr. ir. Y.H. Wijnant 


\section{Graduation committee}

Chairman and Secretary

Prof. dr. G.P.M.R. Dewulf University of Twente

Supervisor

Prof. dr. ir. A. de Boer University of Twente

Co-supervisor

Dr. ir. Y.H. Wijnant University of Twente

Members

Prof. dr. H. Rice

Trinity College Dublin

Prof. dr.-ing. M. Oeser

University Aachen

Dr. ir. K.N. van Dalen

Delft University of Technology

Prof. dr. S. Luding

University of Twente

Prof. dr. ir. D.J. Schipper

University of Twente 



\section{Summary}

The sound radiation caused by tyre/road noise can be reduced significantly by the use of porous road surfaces. A hybrid analytical/numerical modelling approach has been developed to predict the sound absorption coefficient of such road surfaces. Furthermore, the modelling approach has been used as a design tool to optimise the sound absorption of porous road surfaces in the design phase. Using this design tool, two porous surfaces have been developed and constructed at a special test area. These road surfaces have been measured extensively for both sound absorption and for noise radiation in combination with different tyres. This research is carried out within the project 'Silent and Safe Road Traffic'. The goal of this project was to find methods and measures to reduce the noise from tyre/road interaction while ensuring (wet) grip.

The developed hybrid analytical/numerical modelling approach is based on the combination of the solutions of two subsystems: an analytically described background sound field and a numerically solved scattered sound field describing the scattering of the sound waves on the (assumed rigid) porous structure. Furthermore, the sound absorption caused by viscothermal effects inside the air-filled pores is included analytically in the modelling approach. Also, the sound absorption coefficient for oblique incidence can be predicted using this modelling approach. This is an important property when considering tyre/road noise, since most traffic noise is received at oblique incidence. Therefore, the sound absorption for oblique incidence should be considered when predicting the noise reduction by porous road surfaces.

The main advantage of the developed hybrid modelling approach compared to a full numerical model is the low computation time, because (1) no mesh refinement is needed for the mesh of the structure inside the air-filled pores, since the viscothermal effects inside the pores are included analytically, and (2) the air domain surrounding the structure can be relatively small, since the scattering problem is localised around the porous structure and the background sound field is included analytically.

In addition, the developed modelling approach can be applied to predict the sound absorption for any three-dimensional porous structure. The work presented here focuses on structures of tube resonators and on granular structures. Both types of porous structures are used for the validation of the modelling approach. For normal incidence, the modelling approach is validated using the impedance tube technique. The correlation between the measured sound absorption coefficient and the predicted sound absorption coefficient was extremely good for both the tube resonators and structures of stacked glass marbles.

To validate the modelling approach for oblique incidence, a large sound hard box filled with glass marbles was measured using a small cubic microphone array. This validation was more complex, since the measurement technique introduced var- 
ious uncertainties. However, the model results and measurement results showed good correlation.

Furthermore, the developed modelling approach was adjusted in such a way that structures made from sound absorbing materials can be modelled as well. To demonstrate this, a structure of coupled tube resonators has been designed and manufactured with the 3D printing technique, an upcoming technique suitable for manufacturing complex sound reducing panels. The measured sound absorption coefficient of this sample showed an influence of the material properties on the sound absorption coefficient, which could be predicted fairly well with the adjusted modelling approach. 


\section{Samenvatting}

Een succesvolle maatregel om verkeerslawaai te reduceren is het gebruik van poreus asfalt. Om de geluidsabsorptie van zulke poreuze wegen te voorspellen is een hybride analytische/numerieke modelmethode ontwikkeld. Deze modelmethode kan ook worden gebruikt bij het ontwikkelen van nieuwe wegen door in de ontwerpfase de geluidsabsorptie te optimaliseren. Met behulp van de resultaten van de hybride modelmethode zijn twee prototype wegen ontwikkeld en aangelegd. Beide prototype wegen zijn uitgebreid getest, er zijn zowel metingen van de geluidsabsorptie als van de geluidsafstraling in combinatie met verschillende banden gedaan. Dit onderzoek is uitgevoerd als onderdeel van het project 'Stil Veilig Wegverkeer'. Het doel van dit project is om methodes en maatregelen te ontwikkelen om zowel de geluidsoverlast van band-wegverkeer te reduceren als om de (natte) grip te behouden en zo mogelijk te verbeteren.

De ontwikkelde hybride modelmethode is gebaseerd op de combinatie van de oplossing van twee subsystemen: een analytische beschrijving van een achtergrond geluidsveld en een numeriek oplossing van een geluidsveld dat de reflecties van de geluidsgolven op de poreuze (en stijf veronderstelde) structuur beschrijft. In deze methode is ook de geluidsabsorptie door de viskeuze en thermische effecten in de met lucht gevulde poriën meegenomen door middel van analytische beschrijvingen. Met deze modelmethode kan zowel de geluidsabsorptie voor loodrecht invallende golven als de absorptie voor schuin invallende golven worden voorspeld. Dit laatste is een belangrijke eigenschap wanneer de modelmethode wordt gebruikt als ontwerptool om de geluidsabsorptie van poreuze wegen te optimaliseren, zodat het verkeerslawaai voor schuine inval gereduceerd kan worden. Dit is relevant, aangezien verkeerslawaai vooral onder een hoek wordt waargenomen.

Het belangrijkste voordeel van de ontwikkelde hybride modelmethode is de korte rekentijd in vergelijking met een volledig numeriek model. De rekentijd is relatief kort, omdat (1) er geen mesh verfijning nodig is in en rondom de poriën en (2) het lucht domein rondom de poreuze structuur klein kan blijven. Er is geen mesh verfijning nodig, aangezien het viskeuze en thermische gedrag van de lucht in de poriën analytisch wordt opgelost. Het tweede voordeel komt door het lokale gedrag van de reflecties op de (geluidsharde) structuur en doordat de rest van het geluidsveld (het zogenaamde achtergrond geluidsveld) analytisch wordt bepaald.

De ontwikkelde hybride modelmethode kan gebruikt worden om de geluidsabsorptie te voorspellen van allerlei driedimensionale poreuze structuren. In dit proefschrift is geconcentreerd op structuren van buisjesresonatoren en gestapelde knikkers. De modelaanpak is gevalideerd met behulp van beide types structuren voor zowel loodrecht invallende geluidsgolven als schuin invallende golven. De validatie voor loodrechte inval is uitgevoerd met behulp van impedantiebuis metingen. De gevonden correlatie tussen de metingen en modelresultaten was excellent, voor zowel de buisjesresonatoren als structuren met verschillende stapelingen van glazen knikkers. 
Voor de validatie voor schuine inval is een geluidsharde box gevuld met glazen knikkers gebruikt. De metingen zijn uitgevoerd in een vrij geluidsveld met behulp van een driedimensionale intensiteitssonde met 8 microfoons. Deze meetmethode introduceert diverse meetonzekerheden, wat de validatie heeft bemoeilijkt. Desondanks komen de meetresultaten en de modelresultaten (redelijk) goed met elkaar overeen.

Een opkomende productiemethode die veel vrijheden geeft bij het ontwerpen van geluid reducerende panelen zijn de verschillende 3D print technieken. Een van de eigenschappen van de geprinte structuren is de geluidsabsorptie van het materiaal zelf. Daarom is de modelmethode uitgebreid met de mogelijkheid om structuren van geluidsabsorberende materialen te modeleren. Ter validatie is een structuur van meerdere gekoppelde buisjesresonatoren ontworpen en gemaakt met een 3D print techniek. Metingen van de geluidsabsorptie van deze structuur laten de invloed van de materiaaleigenschappen zien, die vervolgens goed voorspeld konden worden met het aangepaste model. 


\section{Contents}

I Extended summary 1

1 Introduction 3

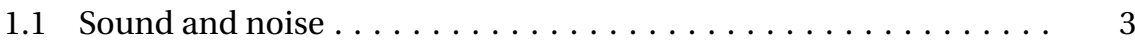

$1.1 .1 \quad$ Tyre $/$ road noise $\ldots \ldots \ldots \ldots \ldots \ldots \ldots \ldots \ldots \ldots$

1.1.2 Silent and Safe Road Traffic . . . . . . . . . . . . . 4

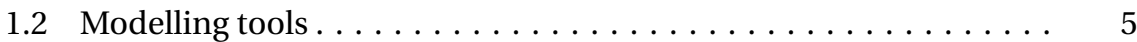

1.2.1 Predicting sound radiation of a rolling tyre $\ldots \ldots \ldots 6$

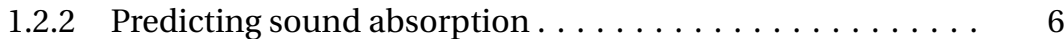

1.2.3 Sound waves and propagation $\ldots \ldots \ldots \ldots$

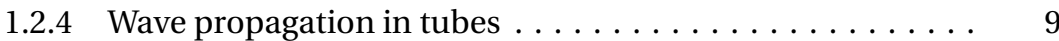

1.2.5 Viscothermal wave propagation $\ldots \ldots \ldots \ldots \ldots$

1.2.6 Sound absorption in porous materials . . . . . . . . . 11

1.2.7 Locally and non-locally reacting surfaces $\ldots \ldots \ldots 11$

1.3 Test area for tyre/road contact: Twente Airport . . . . . . . . . . . . 12

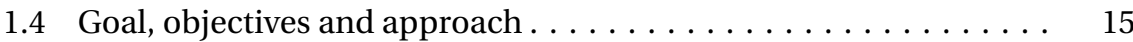

2 Hybrid modelling approach $\quad 17$

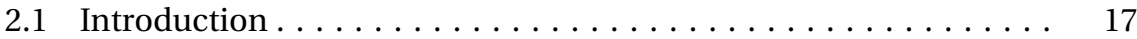

2.1.1 Advantages of a combined modelling approach . . . . . . . 17

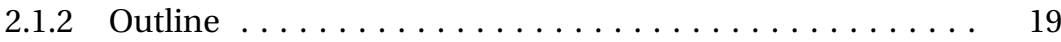

2.2 One-dimensional incident plane waves . . . . . . . . . . . . 19

2.2.1 Hybrid modelling approach $\ldots \ldots \ldots \ldots 19$

2.2 .2 Tube resonators $\ldots \ldots \ldots \ldots \ldots \ldots \ldots \ldots \ldots \ldots \ldots$

2.2.3 Stacked spheres in impedance tube $\ldots \ldots \ldots \ldots 22$

2.3 Three-dimensional oblique incident waves $\ldots \ldots \ldots 23$

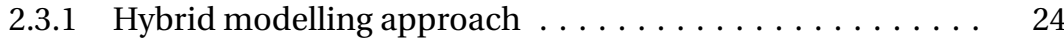

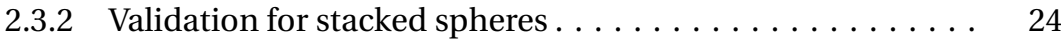

2.4 Design tool for porous road surfaces $\ldots \ldots \ldots \ldots \ldots \ldots \ldots$

2.4 .1 Predictive model $\ldots \ldots \ldots \ldots \ldots \ldots \ldots \ldots \ldots$

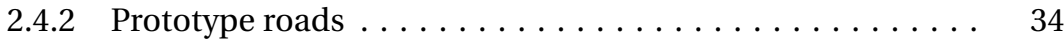

2.4.3 Discussion on design of prototype road surfaces . . . . . . 39 
2.5 Other applications $\ldots \ldots \ldots \ldots \ldots \ldots \ldots \ldots \ldots \ldots \ldots$

2.5.1 Modelling approach extended with impedance of material 41

2.5.2 Validation for $3 \mathrm{D}$ printed sample $\ldots \ldots \ldots \ldots \ldots \ldots$

3 Conclusions and recommendations $\quad 45$

3.1 Conclusions ....................... 45

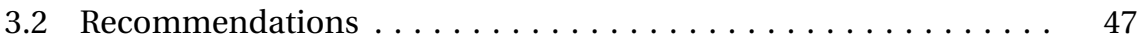

$\begin{array}{lr}\text { Bibliography } & 49\end{array}$

II Papers $\quad 53$

A Predicting sound absorption of stacked spheres: combining an analytical and numerical approach

B Prediction of sound absorption of stacked granular materials for normal and oblique incident sound waves

C Integral approach to tire/road noise: considering both tire and road design

D Modelling approach for 3D printed sound absorbing panels

\section{Appendices}

$\begin{array}{ll}\text { A Properties of air } & 145\end{array}$

$\begin{array}{ll}\text { B Tube resonators } & 147\end{array}$

$1 \quad$ Introduction . . . . . . . . . . . . . . . . . . . . . . 147

2 Sound absorption coefficient for tube resonators . . . . . . . . . 147

$2.1 \quad$ Single tube resonator . . . . . . . . . . . . . 148

2.2 Tube resonators in parallel . . . . . . . . . . . . 148

2.3 Tube resonators in series . . . . . . . . . . . . 149

3 Aluminum sample with 37 parallel tube resonators . . . . . . . . . 149

4 Printed sample with coupled tube resonators . . . . . . . . . . . 151

C Test area for tyre/road contact: Twente Airport 153

$1 \quad$ Layout of test area . . . . . . . . . . . . . . . . . 153

2 Properties of test tracks . . . . . . . . . . . . . . 153 


\section{Part I}

\section{Extended summary}





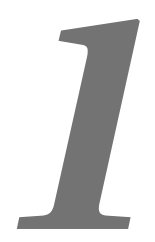

\section{Introduction}

\subsection{Sound and noise}

A truly quiet place is hard to find, since sounds are everywhere. Most people define a quiet place as one without human sounds; sounds caused by nature, such as the wind through leaves, or water dripping into a lake, or animal sounds are often accepted when one seeks silence. A true quiet place would be an anechoic room, designed to absorb all background noise.

In daily life, sounds are often unwanted. These unwanted sounds, generally called noise, are unpleasant and disturbing, stop you from relaxing and sleeping. Constant noise can also cause serious health problems and can even result in death $[29,35,47]$.

Sometimes this noise can be influenced, but often you cannot do anything about it, like noisy neighbours or traffic noise. When effectively reducing noise it is important to understand the complete noise problem from source to receiver, including the path of the noise. In some cases the source of the noise can be insulated directly, for example by adding sound absorbing material inside the casing of the source. For other sources this is more complicated, for example traffic noise, since it is very difficult to insulate the sound source itself.

\subsubsection{Tyre/road noise}

Traffic noise is becoming increasingly important now more and more people are living in densely populated areas and commuting to and from work by car. The main sound sources are engine noise, tyre/road interaction and wind noise caused by the shape of the vehicle. For personal cars driving at a constant cruising speed tyre/road contact is the most important sound source for velocities above $25 \mathrm{~km} / \mathrm{h}$, [49]. Below $25 \mathrm{~km} / \mathrm{h}$, the noise of the power unit is dominant. for much higher velocities, about $100 \mathrm{~km} / \mathrm{h}$, the wind noise is often dominant. An example of the sound radiation caused by tyre/road noise is shown in Figure 1.1. Here, the sound level measured at positions close to the tyre according to the close-proximity measurements [3] for car driving at a constant cruising velocity of $80 \mathrm{~km} / \mathrm{h}$ is shown. The tyres were standard reference tyres (SRTT) and the road 
surface a reference road surface (ISO/R117) with very low sound absorption. The maximum sound level is seen around $900 \mathrm{~Hz}$. Typically, the highest sound levels for tyre/road noise are seen in the frequency range between 800 and $2000 \mathrm{~Hz}$.

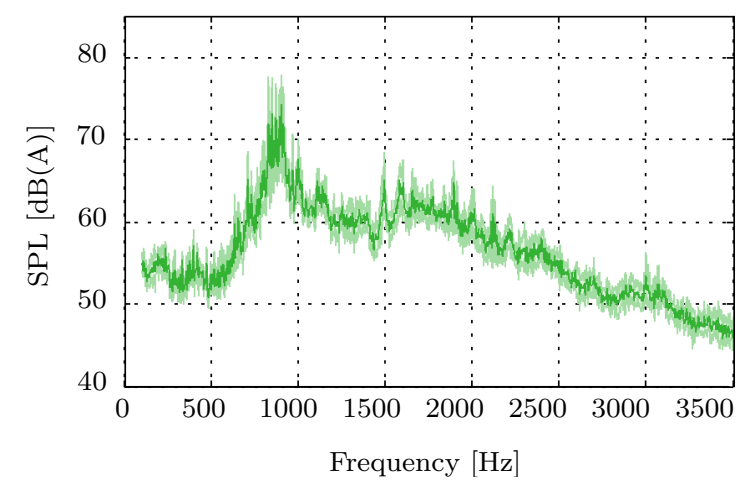

Figure 1.1: Continuous spectrum density of a standard reference tyre (SRTT) on a standard reference road surface (ISO/R117), measured close to the tyre (close-proximity measurement, [3]) with a driving speed of $80 \mathrm{~km} / \mathrm{h}$. The shaded region indicates a \pm 1 standard deviation.

Knowledge about the noise mechanisms and noise paths from tyre/road contact to the ear of the receiver are very important to reduce traffic noise caused by tyre/road contact. More information about those mechanisms can be found in $[9,32,49]$. Extensive research has been carried out to find the influence of tyre properties on the excitation mechanisms, using simple and more complex models. For example models focusing on the tyre tread design [9], on the stiffness properties of the tyre or on the tyre resonance frequencies [36], or more complex models combining multiple mechanisms [25, 34, 40, 51].

The sound excitation mechanisms depend also on the road surface and can be minimised by designing a silent road, for example one with a smooth or elastic road surface, such that excitations are minimised, [48]. Secondly, the radiated noise can be (partly) absorbed, for example by a porous asphalt road surface $[13,48]$. Furthermore, the combination of elastic and porous road surfaces, the so-called poroelastic road surfaces $[20,50]$, is also possible, though many practical problems remain.

And thirdly, the noise can be blocked by noise barriers or houses with special structures or materials for the facade, for example noise barriers integrated with the façade of the houses or silent façades with few or no windows at the side of the $\operatorname{road}[27,39]$.

\subsubsection{Silent and Safe Road Traffic}

To regulate and reduce the traffic noise, the European Union has introduced labelling for the tyres [1], and most national/local governments take measures to re- 
duce the noise radiation from traffic, for example by constructing absorbing road surfaces or noise barriers. To find methods and measures to reduce the noise from tyre/road interaction while ensuring grip, the project SVW ('Silent and Safe Road Traffic', in Dutch: 'Stil Veilig Wegverkeer') was initiated and funded by the 'European Regional Development Fund', the Twente Region and the Province of Overijssel. Important partners are the Province of Gelderland, the University of Twente (Tire-Road Consortium), Apollo Vredestein B.V., Reef Infra and Stemmer Imaging.

The main objective of this project was to research the tyre/road contact, with three focus areas: safety, sustainability and environment, as indicated in Figure 1.2 and described by Bekke et al. [11]. An important issue was the focus on the integral approach to optimise the roads and tyres for both noise and grip.

Other important objectives were to develop simulation tools [41], perform measurements and develop or improve test procedures. The main deliverables of the project were prototypes for both tyre and road, optimised for wet grip and noise.

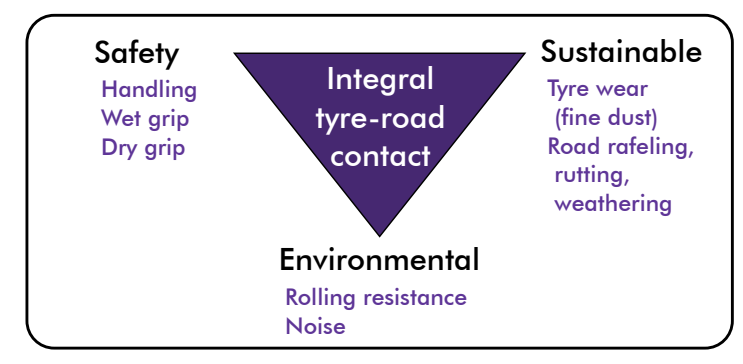

Figure 1.2: Integral approach to tyre/road contact.

Within this project, several companies and $\mathrm{PhD}$ researchers have been working together to find methods to predict and test the tyre/road contact. Bekke [9] worked on tools to predict interior noise caused by the tyre/road contact and Mokhtari [43] has researched the physical contact between tyre and road, focusing on the contact and friction of rubber. The present work, concentrating on predicting and optimising the sound absorption of porous asphalt roads, was also carried out within the SVW project.

\subsection{Modelling tools}

The development of modelling tools to predict tyre/road noise is one of the objectives of the SVW project. The road has an important role in both sound absorption properties and noise generating mechanisms. In particular, the road roughness and the mechanic impedance of the road have a large influence on the generated noise [48]. Therefore, when optimising the road surface for reduction of traffic noise, both the sound absorption properties and the sound generating properties of the road should be taken into consideration. 


\subsubsection{Predicting sound radiation of a rolling tyre}

The influence of the tyre tread pattern and the road roughness can be found using the Tyre Road Noise (TRN) model, described by [51]. With this numerical model, the sound radiation for a rolling tyre can be predicted fairly well. The model consists of a structural model, where the dynamic and structural vibrations of the complete rolling tyre are predicted in a transient simulation, using the FEM package Abaqus. The vibrations at the tyre surface are then mapped onto a static boundary elements method (BEM) mesh to predict the sound radiation of the rolling tyre. The model is rather extensive and includes the tyre tread design, the material properties, a simple contact model for the tyre/road contact and statistical information about the road roughness. However, the sound absorption of the road surface is implemented in the TRN model in a very limited manner:

- The sound absorption coefficient is constant over frequency.

- The sound absorption coefficient does not depend on the angle of incidence of the sound waves, which is relevant since rolling tyres radiate noise in all directions. Especially grazing incidence should be considered, when researching the effect of tyre/road noise on the environment, as is indicated in Figure 1.3.

This limited implementation could explain the differences seen between the model results and the measurements of the sound radiation at the so-called CPX positions. These are the locations of the microphones during the close-proximity (CPX) measurements, [3].

Therefore, the focus of the present work has been on developing a modelling approach to predict the sound absorption of the porous road surface for normal and oblique incident sound waves, which can be combined with the existing TRN model for more accurate predictions of the sound radiation of a rolling tyre.

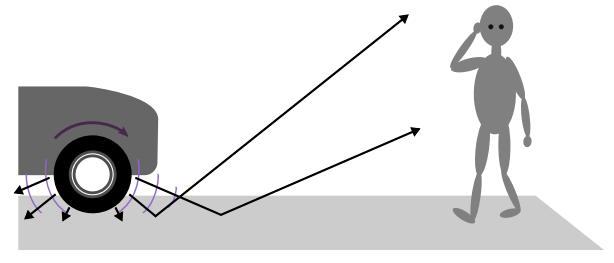

Figure 1.3: Illustration of sound radiation for tyre/road noise.

\subsubsection{Predicting sound absorption}

Porous asphalt concrete can be considered as a sound absorbing material. The small air-filled pores in the bitumen and stone matrix partly absorb tyre/road noise. The sound absorption behaviour depends for example on the layer thickness, the porosity, the stone sizes and stone shapes.

The sound absorption of road surfaces is usually measured after manufacturing. 
However, when optimising the road surface for the reduction of sound radiation of tyre/road contact, it would be more effective to predict the acoustical characteristics of the road surface beforehand. Various empirical and analytical models are available, the so-called ground impedance models [7]. A distinction can be made between (semi-)empirical models, phenomenological or macrostructural models and microstructural models. These models range from relations based on measurement results to complex analytical relations based on the microstructure of the porous material or structure. Overviews of the different categories of these models and some examples are given by $[4,6,7]$. Also, a short overview is given in Paper A (Predicting sound absorption of stacked spheres: combining an analytical and numerical approach, [15]).

Furthermore, applying sound absorbing materials for noise insulation and sound reduction is very common and models not specifically developed to describe porous asphalt concrete could also be used for this application.

\section{Examples of porous materials and sound reducing structures}

A well-known type of porous material used for sound insulation is glass wool or foam, shown in Figure 1.4. The main sound absorption mechanisms are the viscous and thermal losses due to the small diameter and winding path of the airfilled pores inside these materials. Another sound absorption mechanism is the interaction between the flexible bulk material and the air-filled pores. Many studies have been performed to describe the sound absorption of such materials, varying from simple 2 parameter empirical models like the Delany and Bazley model [28] to more complicated models describing the entire microstructure using analytical equations for the viscous and thermal energy dissipation inside the airfilled pores, for example [26, 33, 52-54, 57]. Often these models that describe the behaviour of the air inside the pores are combined with the Biot model to include the behaviour of the bulk material $[4,5,22,23]$. This bulk material can be modeled rigid and flexible.

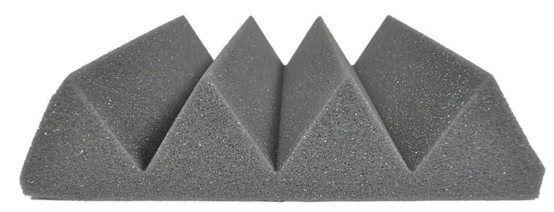

Figure 1.4: Sound absorbing foam [8]

Examples of sound reducing structures are the periodic absorbers (Figure 1.5a) or reflecting structures with resonators, such as a panel with tube resonators (Figure 1.5b). These structures can be used for broadband sound absorption or can be tuned to absorb specific frequencies. Again, many studies can be found describing the acoustic behaviour of this type of sound reducing structure. For instance, [42] developed an analytical method to describe periodic absorbers with spatial harmonics, focusing on the pressure field above these periodic structures, while 


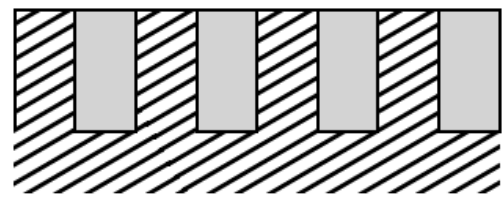

(a) Periodic absorber [37]

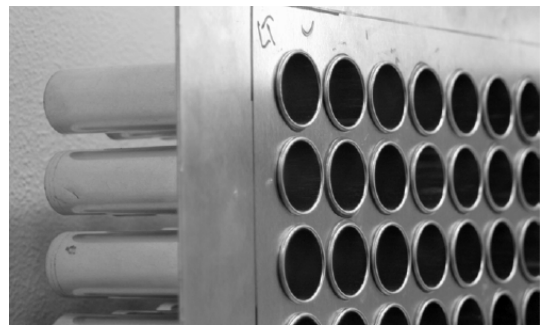

(b) Panel with tube resonators [30]

Figure 1.5: Examples of sound absorbing materials and structures

[56] and [30] concentrated on describing (structures of) tube resonators with analytical relations for the sound field inside these tubes, using the LRF model.

\subsubsection{Sound waves and propagation}

Sound consists of longitudinal waves propagating through the bulk of a medium, such as air or water. A sound wave travels in space and in time and can be described by the wave equation, derived from the conservation equations for fluids $[21,24]$.

The general form of the (linear) wave equation for sound waves is:

$$
\nabla^{2} p-\frac{1}{c^{2}} \frac{\partial^{2} p}{\partial t^{2}}=0
$$

where $t$ is a time variable, $p$ is the pressure of the wave, $\nabla$ is the (spatial) Laplacian and $c$ is the speed of sound. A general time-harmonic solution for the wave equation for a travelling wave in negative z-direction, as indicated in Figure 1.6, is:

$$
p_{A}=A e^{i(\omega t+k z)}
$$

where $k=\omega / c$ is the wave number, $\omega$ is the angular frequency, $i$ is the imaginary quantity for which $i=\sqrt{-1}$ and $A$ is the (complex) amplitude of the wave. For a wave with amplitude $B$, travelling in the positive z-direction, the general solution yields:

$$
p_{B}=B e^{i(\omega t-k z)}
$$

A distinction can be made between travelling waves and standing waves, where a standing wave can be constructed from Equation 1.2 and 1.3 by adding a forward travelling wave and a backward travelling wave, for which the amplitude is $B=A$.

For the remainder of this dissertation, the time dependence $e^{i \omega t}$ is omitted. The directions and conventions used throughout this dissertation are indicated in Figure 1.6. 


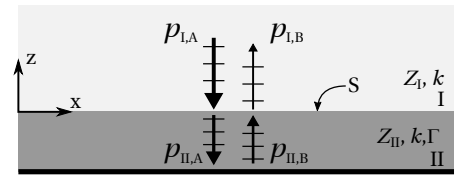

Figure 1.6: Direction and definitions of incident, transmitted and reflected sound waves on the interface $S$ between medium I and II and reflected at the sound hard backing of medium II, where medium I and medium II have different properties.

\subsubsection{Wave propagation in tubes}

In Figure 1.7, sound waves propagating through a cylindrical tube with sound hard boundaries are shown. There are only plane waves propagating in the longitudinal direction of the tube, as long as the maximum frequency range is below the cutoff frequency of the tube with diameter $D$, which is given by $f_{c o}=c /(1.7 D)$. The sound pressure inside the tube is given by the solution for the Helmholtz equation assuming time-harmonic plane waves:

$$
p=A e^{i k z}+B e^{-i k z}
$$

where the forward travelling wave is travelling in the negative $\mathrm{z}$-direction and the backward travelling waves in the positive $z$-direction. The particle velocity $u$ is given by:

$$
u=\frac{-1}{\rho_{0} c_{0}}\left(A e^{i k z}-B e^{-i k z}\right)
$$

where $\rho_{0}$ is the density of air and $c_{0}$ is the speed of sound in air, both assuming standard air conditions. The complex amplitudes $A$ and $B$ are determined by the boundary conditions at both ends of the tube. The quantity $\rho_{0} c_{0}$ is usually called the characteristic impedance $Z_{0}$ of air. Since only plane waves are considered here, the characteristic impedance depends only on the properties of the medium and not on the waveform itself. Note that the characteristic impedance of air describes the impedance for an undisturbed propagating wave; a freely travelling wave.

The (specific) acoustic impedance $Z$ describes the relation between the pressure and velocity at a specific position, $[24,56]$ :

$$
Z \equiv \frac{p}{u}
$$

The (specific) impedance can be a complex quantity, indicating a phase difference between the pressure and particle velocity.

\subsubsection{Viscothermal wave propagation}

For tubes with a small radius or for layers with a small thickness, the wave propagation is affected by loss mechanisms, such as the viscosity and the thermal conductivity of the medium inside the tube. This viscothermal wave propagation is 


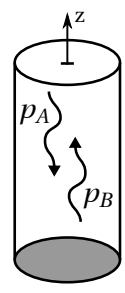

Figure 1.7: Directions and definitions of incident and reflected sound waves in a tube with one open end and one closed end.

extensively treated in the literature, for example in [12] and [54]. The approach followed in this work is based mainly on the so-called low reduced frequency (LRF) model [12, 30, 46, 54, 56, 57]. Using the LRF approach, analytical solutions are derived for the sound field inside and above a prismatic tube, including effects of inertia, compressibility, viscosity and thermal conductivity of the medium. The main assumptions and relations for the LRF model are discussed in detail in $[12,30,46,54,56,57]$. The relations for the (viscous) air inside a prismatic tube with a circular cross-section are summarised below.

In the LRF modelling approach, the viscothermal wave propagation coefficient is indicated by $\Gamma$, yielding:

$$
\Gamma=\sqrt{\frac{J_{0}(i \sqrt{i} s)}{J_{2}(i \sqrt{i} s)} \frac{\gamma}{n}}
$$

where $J_{0}$ and $J_{2}$ are the Bessel functions of the first kind of order 0 and 2, $s$ is the shear wave number, $\gamma$ is the ratio of specific heats at constant pressure $C_{p}$ and constant volume $C_{V}, N_{\mathrm{Pr}}$ is the Prandtl number and $n$ is the polytropic coefficient:

$$
\begin{array}{r}
s=r_{t} \sqrt{\frac{\rho_{0} \omega}{\mu}} \text { and } \gamma=\frac{C_{p}}{C_{V}} \text { and } N_{\mathrm{Pr}}=\frac{\mu C_{p}}{\lambda} \\
n=\left[1+\frac{\gamma-1}{\gamma} \frac{J_{2}\left(i \sqrt{i N_{\mathrm{Pr}}} s\right)}{J_{0}\left(i \sqrt{i N_{\mathrm{Pr}}} s\right)}\right]^{-1}
\end{array}
$$

where $\mu$ is the dynamic viscosity and $\lambda$ is the thermal conductivity.

The viscothermal wave propagation coefficient $\Gamma$ is related to the wave number by $k=-i k_{0} \Gamma$ and depends on the shape of the pore, the angular frequency and the wave number assuming standard air, $k_{0}$. The relations for the complex pressure and velocity inside a prismatic tube are:

$$
\begin{aligned}
& p=A e^{\Gamma k z}+B e^{-\Gamma k z} \\
& u=\frac{G}{\rho_{0} c_{0}}\left[A e^{\Gamma k z}-B e^{-\Gamma k z}\right]
\end{aligned}
$$

where $\rho_{0} c_{0} / G$ can be considered as the characteristic impedance $Z$ of the medium inside this tube. 
The coefficient $G$ depends on the angular frequency, the properties of the medium and the shape of the cross-section of the tube. For a circular cross-section, this coefficient yields:

$$
G=-\frac{i}{\Gamma} \frac{\gamma}{n}
$$

\subsubsection{Sound absorption in porous materials}

In general, the dissipation of sound in a material or medium is caused by loss mechanisms such as viscosity or thermal conduction. The result is often heat generation and therefore a loss of energy, which results in the absorption of sound. Absorption of sound depends on the frequency, which is caused by the frequency dependent speed of sound and is called dispersion [24]. The dispersion of energy due to these loss mechanisms, has to be included in the conservation equations.

For plane waves incident at the interface $S$ between two mediums, part of the waves will be reflected and part of the waves will be transmitted through the second medium (as indicated in Figure 1.6). The ratio between the incident and reflected waves is usually expressed by the reflection coefficient $R$ [24]:

$$
R=\frac{p_{B}}{p_{A}}=\frac{B}{A}
$$

This relation is based on two conditions: (1) the pressure on both sides of the interface must be the same and (2) the normal component of the particle velocity must be continuous across the interface. Applying these boundary conditions and using Equation 1.6, the reflection coefficient can be written as:

$$
R=\frac{Z_{\mathrm{II}}-Z_{\mathrm{I}}}{Z_{\mathrm{II}}+Z_{\mathrm{I}}}
$$

where $Z_{\mathrm{I}}$ is the characteristic impedance of medium I and $Z_{\mathrm{II}}$ the characteristic impedance of medium II [24].

The frequency dependent sound absorption coefficient $\alpha$ for a surface describes the fraction of the energy that is dissipated. For a plane wave, the acoustic energy is proportional to the squared amplitude of the wave, hence:

$$
\alpha=\frac{|A|^{2}-|B|^{2}}{|A|^{2}}=1-|R|^{2}
$$

Since the (complex) amplitude of the incident and reflected wave, as well as the impedance of both mediums, depends on the angular frequency $\omega$, the sound absorption coefficient is frequency dependent.

\subsubsection{Locally and non-locally reacting surfaces}

Depending on the porous material and the surface of the porous material, the surface impedance can be determined based on the angle of incidence and acoustic behaviour of the material. Three different categories of surface behaviour are illustrated in Figure 1.8, where the porous material is indicated with II. 
When the surface impedance does not depend on the angle of incidence and the location of the incident waves at the surface, the material behaves as a locally reacting surface, as illustrated in Figure 1.8a. A locally reacting surface can be modelled as an impedance plane using Equation 1.6 with $u=u_{z}$, the component of the particle velocity in the $\mathrm{z}$-direction.

For a homogeneous material, the behaviour can be described as an extendedreaction material, illustrated in Figure 1.8b. In this case, the refraction of sound waves depends on the angle of incidence, the impedance of the material and the thickness of the layer. Therefore, the normal surface impedance depends not only on the physical properties of the porous material, but also on the angle of incidence.

The surface impedance $Z_{s}$ for an extensively reacting material with acoustical hard backing can be derived using:

$$
Z_{s}=\frac{-i Z_{c}}{\tan (k d)}
$$

where $d$ is the layer thickness of the porous material and $k^{2}=k_{x}^{2}+k_{y}^{2}+k_{z}^{2}$ is the wave number including the direction of the incoming waves [4].

It is more complicated when the surface impedance of a material depends on the location and on the angle of incidence of the incoming acoustic pressure. An example of this non-locally reacting behaviour is shown in Figure 1.8c. Note that only the last category includes scattering behaviour.

Porous asphalt concrete is often described in the literature as an extensively reacting surface with acoustically hard backing, for instance in [7, pp. 39-46]. In this work, porous asphalt concrete is modelled as a non-locally reacting surface, such that behaviour such as scattering and sound absorption inside the asphalt concrete are included as well.

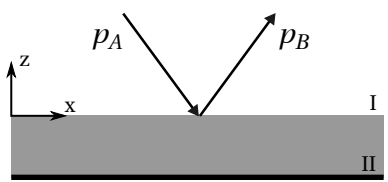

(a) Locally reacting

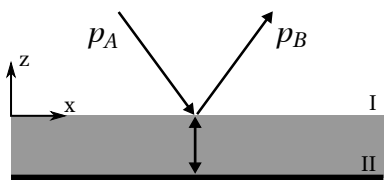

(b) Extensively reacting

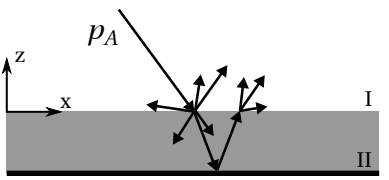

(c) Non-locally reacting

Figure 1.8: Schematic representation of three categories of acoustical behaviour of surfaces for an incident wave $p_{A}$ and reflected wave $p_{B}$. The porous material is indicated with II and the air above with I.

\subsection{Test area for tyre/road contact: Twente Airport}

The main measurement location used for this research is a special test site developed within the project 'Silent and Safe Road Traffic'. This special test site was located at Twente Airport, an out-of-service airport situated in a quiet and rural area in the eastern part of the Netherlands. A total of eight different tracks were constructed at this location. The first six test tracks were manufactured in 2013 


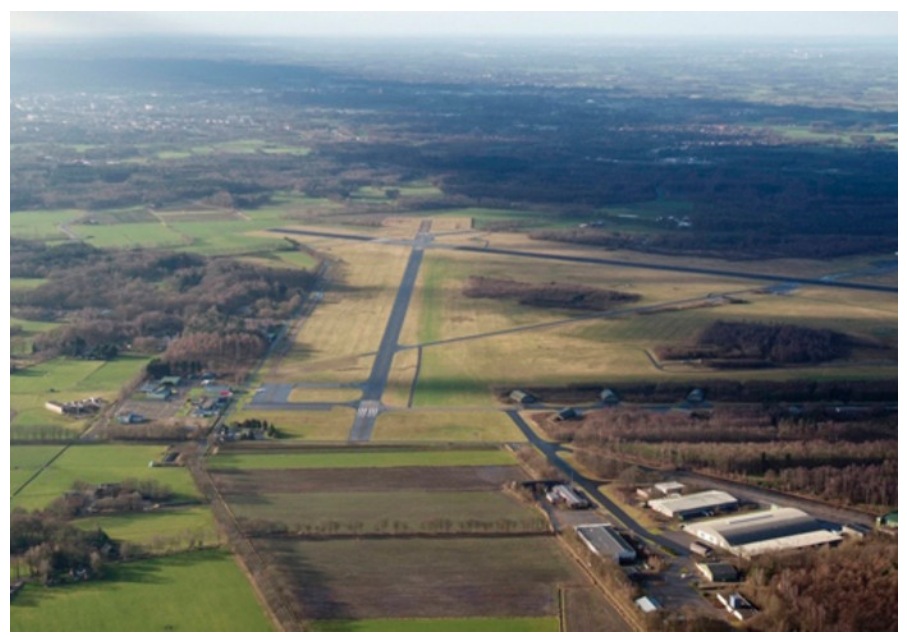

Figure 1.9: Twente Airport from the air [55].

and measured extensively. These measurement results, together with the simulation tools developed within the SWW project, have been used to design two prototype road surfaces which were manufactured in 2015. More information about the properties of these test tracks can be found in Appendix C.

An impression of the test site at Twente Airport is shown in Figures 1.9 and 1.10.

The composition of first six test tracks was in accordance with currently used road surfaces with diverse characteristics, such as porosity, type and size of stone. Each track was $3 \mathrm{~m}$ wide and $100 \mathrm{~m}$ in length. A photo of the construction of the test tracks is shown in Figure 1.11.

Various measurements were performed at these test tracks within the project SVW by researchers, companies and institutes. The main focus of these measurements was on determining the (wet) grip and sound radiation of different combinations of tyres and road surfaces.

The grip measurements at these tracks were made by Milad Mokthari of the University of Twente in collaboration with the company Apollo Vredestein B.V. This resulted in $[43,44]$, detailing the physical contact between tyre and road, focusing on the contact and friction of rubber. Furthermore, two special 3D scanners were developed to map the micro structure of the road surfaces. The scanners were developed by Stemmer Imaging.

The noise measurements at these test tracks served several goals, the work of [9] and the present work being the main focal points. Dirk Bekke researched the interior noise radiation of the tyre/road contact. This resulted in different tools to predict interior noise caused by the tyre/road contact and to aid the design of new (silent) tyre tread patterns $[9-11,41]$.

The research described in the present work focuses on the exterior tyre/road 


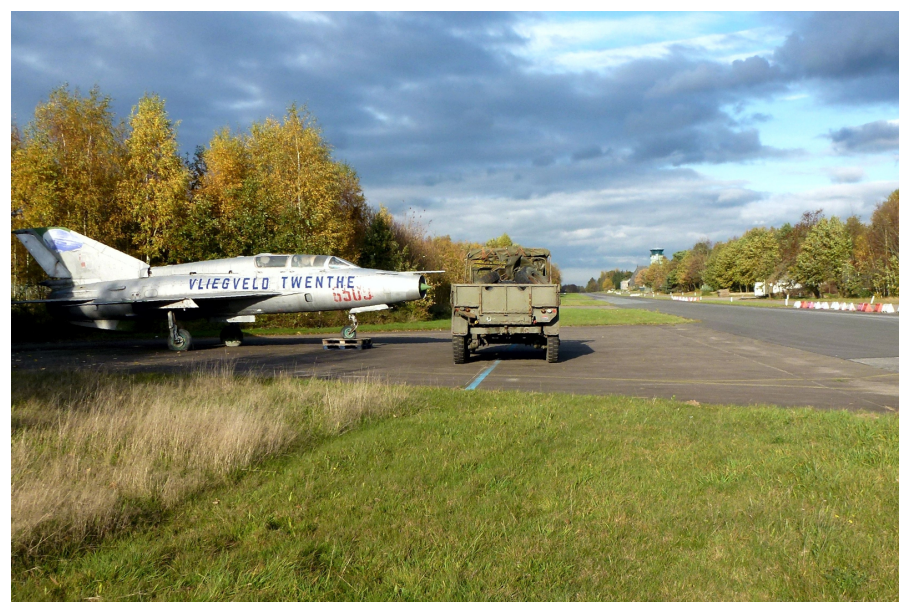

Figure 1.10: Twente Airport, a former military airport in the eastern part of the Netherlands.

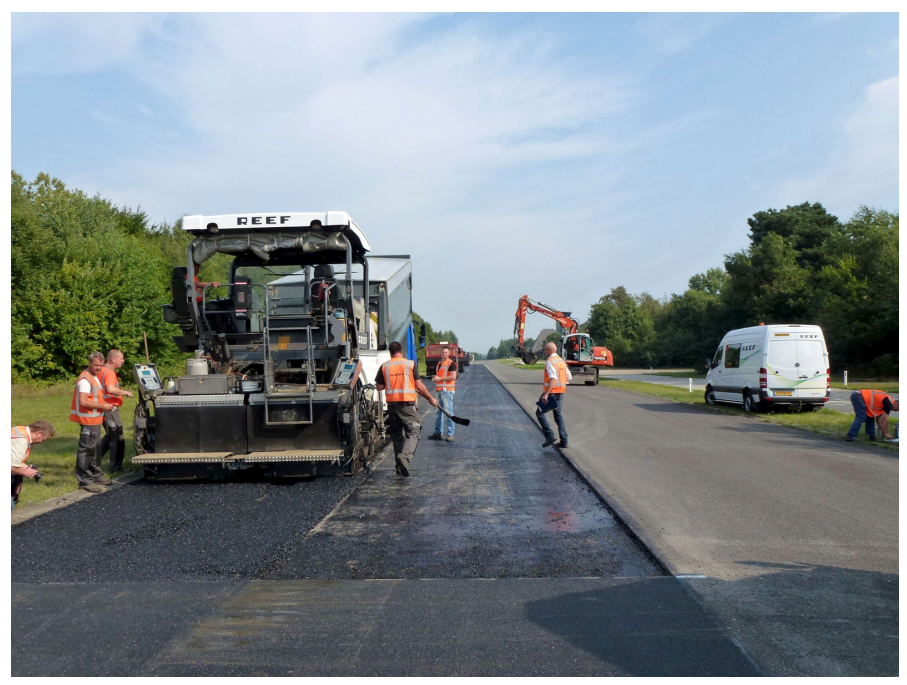

Figure 1.11: Construction of the first six test tracks in 2013 at Twente Airport.

noiseand, more specifically, on developing a modelling tool to predict the sound absorption coefficient of porous materials, such as porous asphalt roads. The goals of this research within the SVW project were to gather knowledge and develop tools to aid the design of silent roads [15-17, 19]. This research was carried out mostly at the University of Twente, aided by the companies Apollo Vredestein B.V. and Reef Infra.

These measurements and predictive models and design tools resulted in the development of two new road surfaces, developed and constructed in 2015. Both 
new surfaces were optimised for best grip and minimum noise radiation. Furthermore, the performance of these tracks was measured both in situ and in the laboratory, using slabs of the test tracks. The design process and measurement results for exterior noise radiation are included in this dissertation and the papers $[16,17,19]$. The in-situ measurements included close-proximity (CPX) measurements, controlled pass by measurements, sound absorption measurements and road roughness measurements. An impression of the pass by measurements is shown in Figure 1.12.

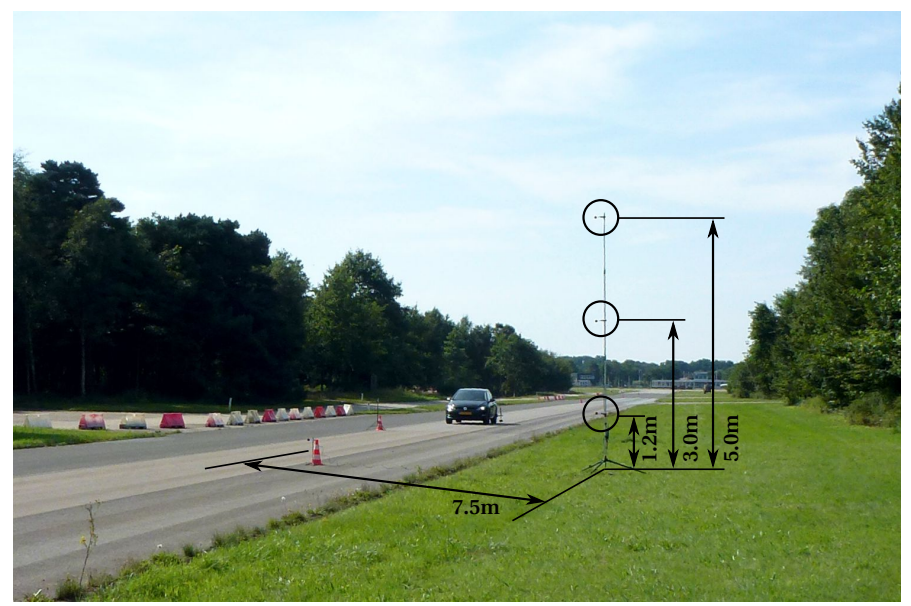

Figure 1.12: Controlled pass by measurements at Twente Airport.

\subsection{Goal, objectives and approach}

This research does not focus only on porous asphalt road surfaces but also takes into account other porous materials. The goal of this study is defined as:

Developing a modelling approach to predict the sound absorption coefficient of porous structures and granular materials for normal and oblique incident sound waves.

This research goal is divided into the following objectives in order to develop and demonstrate such a modelling approach:

- Include the viscothermal effects inside the air-filled pores of the modelled structure to better describe the sound field in and above these structures.

- Include local effects, such as scattering of sound waves on the sound hard elements of the modelled structure to determine the influence of the properties of the stone matrix of the porous asphalt concrete, for example the stone size and shape.

- Validate the modelling approach experimentally for both normal and oblique incident sound waves for three-dimensional structures of gran- 
ular materials, such as a packing of stacked marbles, since such a structure is well-defined and easily modelled.

- Demonstrate how this modelling approach can be used as design tool to optimize the sound absorption of new porous asphalt road surfaces.

- Demonstrate that this modelling approach allows to predict the sound absorption for other applications as well, such as designing acoustic resonators for sound reduction.

When using the developed modelling approach to predict the sound absorption coefficient of porous asphalt concrete, the structure of the asphalt is simplified to a porous granular structure. Then the modelling approach can be used as a design tool to study the influence of the stone size and porosity in asphalt grading, the thickness of the asphalt layer and the effect of multiple layers of different grades. Furthermore, the sound absorption coefficient for oblique incidence can be predicted using this approach.

In Chapter 2 the developed modelling approach is discussed and in Chapter 3 the conclusions and recommendations are given. Furthermore, this dissertation contains four (journal) articles in which the objectives achieved and methods used are discussed in more detail. Figure 1.13 shows a schematic overview of the topics of these articles and the relation between the articles and the objectives. The articles can be found in Part II.

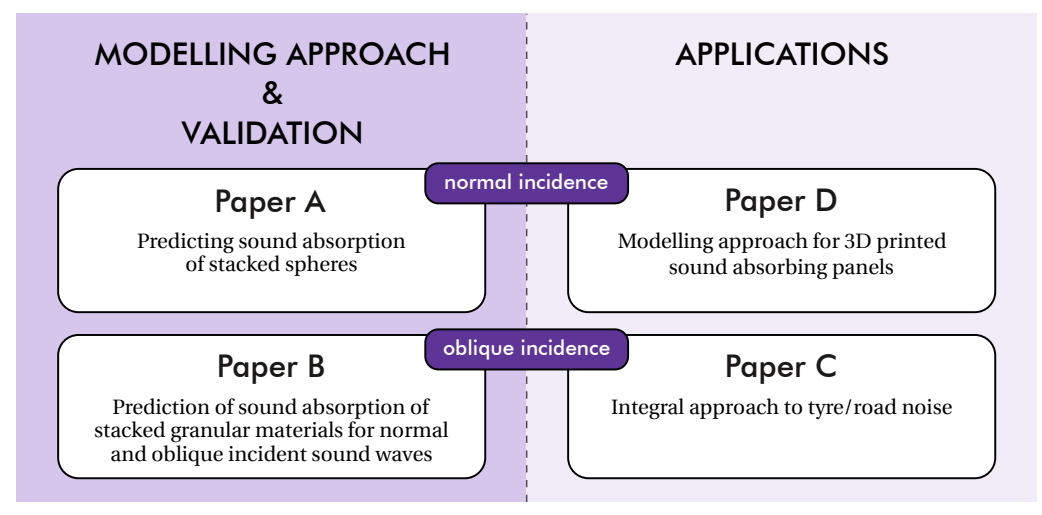

Figure 1.13: Schematic overview of articles in part II. 


\section{2}

\section{Hybrid modelling approach}

\subsection{Introduction}

A hybrid analytical/numerical modelling approach to predict the sound absorption coefficient of porous structures and granular materials for normal and oblique incident sound waves has been developed. This approach includes the viscothermal effects inside the air-filled pores of the structures as well as the local effects, such as scattering of sound waves on the rigid structure. The modelling approach is validated for normal and oblique incidence using tube resonators and structures of stacked marbles. Note that in this hybrid modelling approach only the air in and around the porous structure is considered. The porous structure itself is assumed rigid and sound hard. Examples of modelling approaches which include the sound propagation through the porous structure or the vibrations of the porous structure are given by [5, 22, 23, 45].

To reduce computational time, the total solution for the sound field is found by combining the solutions of two subsystems: a background sound field and a scattered sound field. The modelling approach is shown schematically in Figure 2.1. In the subsystem of the background sound field, the viscothermal behaviour in the air-filled pores of the material is described analytically using a microstructural model; in this case the low-reduced frequency (LRF) model [12, 30, 46, 54, 56, 57]. In the subsystem describing the scattered sound field, the scattering of the incident waves is modelled numerically using a finite element method. The background and scattered field are summed to find the total solution, such that both the viscothermal effects and the scattering effects are included. This is allowed, since both subsystems describe only linear acoustics.

\subsubsection{Advantages of a combined modelling approach}

The main advantage of this modelling approach is the (relatively) low computational time, while the total problem that is solved is quite complex. The computational time is kept low, since (1) the viscothermal behaviour is included analytically and (2) the scattering problem has to be solved only locally around the porous structure. 


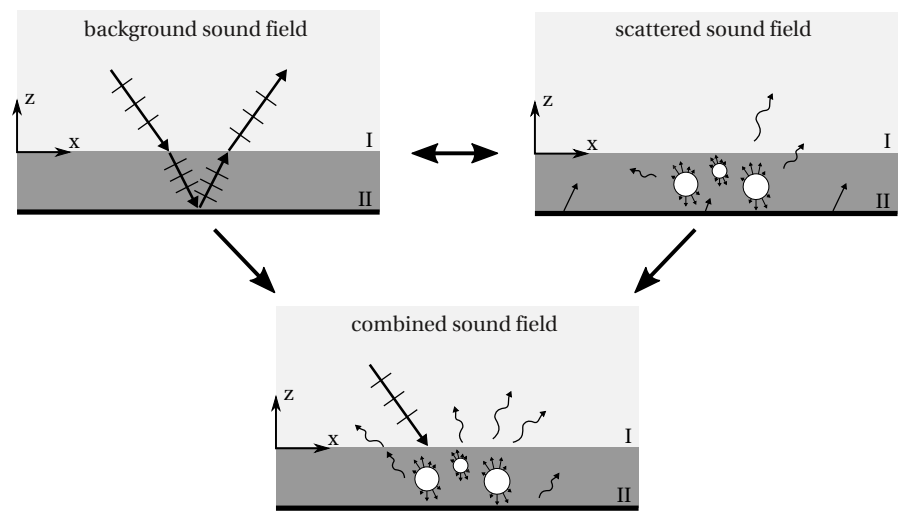

Figure 2.1: Schematic view of hybrid analytical/numerical modelling approach, where two subsystems are combined to find the total solution.

In Figure 2.2a, the velocity profile in a viscous medium near the sound hard boundary of a porous structure is shown. When the viscothermal behaviour is included numerically, the mesh close to the boundaries of the sound hard structure should be fine enough to include the boundary layer effects. However, in this modelling approach, the viscothermal behaviour is included analytically and therefore it is not necessary to refine the mesh close to the boundaries.

The second computational advantage of this modelling approach, is based on the assumption that the scattering of the sound waves is concentrated locally around the porous structure. The rest of the sound field is solved analytically in the subsystem of the background sound field. Therefore, the dimensions of the numerical model can be kept (relatively) small. This is illustrated in Figure 2.2b.

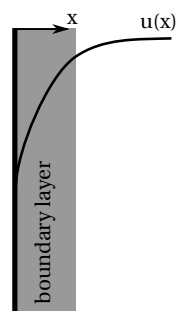

(a) Velocity profile in a viscous air layer close to a sound hard, rigid boundary.

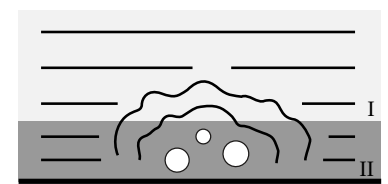

(b) Scattering of sound waves concentrated around the porous structure.

Figure 2.2: Computational advantages of the hybrid analytical/numerical modelling approach. 


\subsubsection{Outline}

The research on developing and validating this hybrid modelling approach is described in more detail in four (journal) articles. An overview of the topics discussed in these articles is shown schematically in Figure 1.13. This chapter contains a brief discussion of the hybrid modelling approach and some of the applications.

Section 2.2 describes the solution for the sound field for one-dimensional incident waves. The modelling approach is explained and validated for a sample with tube resonators inside an impedance tube. Furthermore, the validation of a more complex geometry using stacked marbles is shown in this section and discussed in more detail in Paper A: Predicting sound absorption of stacked spheres: combining an analytical and numerical approach, [15].

In Section 2.3, the solution for the sound field for three-dimensional incident waves is given. For the validation of this three-dimensional problem, the reader is referred to Paper B: Prediction of sound absorption of stacked granular materials for normal and oblique incident sound waves, [19].

Two applications of this modelling approach are discussed in Section 2.4 and Section 2.5. Section 2.4 describes how this modelling approach can be used as modelling tool to optimise the design of porous asphalt road surfaces to reduce tyre/road noise. A different kind of application is given in Section 2.5, where the hybrid modelling approach is used to design 3D printed sound reducing absorbers for broadband sound absorption. This additive manufacturing (AM) technique allows for great freedom in the design of the absorber. Furthermore, to accommodate the additional acoustic properties of 3D printed materials, the hybrid modelling approach is extended with sound absorption by the material itself.

\subsection{One-dimensional incident plane waves}

The hybrid modelling approach consists of two subsystems, both describing the sound field in two layers, as is indicated in Figure 2.1. One layer, medium I, is assumed to have standard air conditions and the second layer, medium II, is a viscous air layer, representing the air inside the porous material $[14,15]$.

\subsubsection{Hybrid modelling approach}

The background sound field is solved analytically, according to the boundary conditions shown in Figure 2.3. Note that medium II represents the viscous air inside the porous material and does not contain the (rigid) structure of the material itself; this is, however, included in the numerical model solving the scattered sound field.

The sound pressure $p_{\mathrm{I}}$ in medium I is given by:

$$
p_{\mathrm{I}}=A_{\mathrm{I}} e^{i k z}+B_{\mathrm{I}} e^{-i k z}
$$

where $A_{\mathrm{I}}$ and $B_{\mathrm{I}}$ are the complex amplitudes of the incident and the reflected waves, respectively, and $k$ is the wave number in this medium, assuming standard air conditions. The particle velocity normal to the interface between medium I 


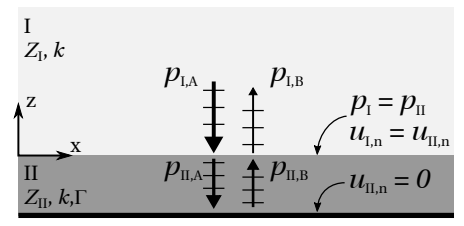

Figure 2.3: Schematic overview of the wave propagation through both mediums and the boundary conditions for the background sound field.

and II is given by:

$$
u_{\mathrm{I}, n}=\mathbf{u}_{\mathrm{I}} \cdot \mathbf{n}=\frac{-1}{Z_{\mathrm{I}}}\left[A_{\mathrm{I}} e^{i k z}-B_{\mathrm{I}} e^{-i k z}\right]
$$

where $\mathbf{u}_{\mathrm{I}}$ is the particle velocity, $\mathbf{n}$ is the unit vector normal to the interface and $Z_{\mathrm{I}}=\rho_{0} c_{0}$ is the characteristic impedance of air.

The sound pressure $p_{\mathrm{II}}$ and the particle velocity $\mathbf{u}_{\mathrm{II}}$ in the viscous air layer, medium II, are given by:

$$
\begin{aligned}
p_{\mathrm{II}} & =A_{\mathrm{II}} e^{\Gamma k z}+B_{\mathrm{II}} e^{-\Gamma k z} \\
u_{\mathrm{II}, n} & =\mathbf{u}_{\mathrm{II}} \cdot \mathbf{n}=\frac{G}{\rho_{0} c_{0}}\left[A_{\mathrm{II}} e^{\Gamma k z}-B_{\mathrm{II}} e^{-\Gamma k z}\right]
\end{aligned}
$$

where $\Gamma=i k_{\mathrm{II}} / k$ is the viscothermal wave propagation coefficient and $G$ a coefficient dependent on the shape of the pores and the angular frequency, both according to the LRF model (Section 1.2.5). Note that the LRF model is similar to a microstructural ground impedance model, and can be replaced by any ground impedance model which includes the viscothermal energy dissipation.

The sound pressure due to the scattering of the incident sound waves on the porous material is solved numerically using the finite element method (FEM). Note that only the air inside the porous material is considered. Therefore, the structure or frame of the porous material, for example a tube resonator, is modelled as accurate as possible and subtracted from the viscous air layer. The frame itself is assumed rigid and acoustically hard.

This subsystem is solved as a typical scattering problem, where the sound radiation due to a normal velocity at the boundaries of the frame of the porous material is solved. In this case, the normal velocity applied to the boundaries equals the particle velocity of the background field at the same position, but in the opposite direction. Therefore, when the solutions of both subsystems are summed to find the solution for the total sound field, the normal velocity $u_{n}$ is equal to zero at all boundaries:

$$
u_{n}=u_{n, \mathrm{bw}}+u_{n, \mathrm{scat}}=0
$$

where $u_{n \text {,bw }}$ is the particle velocity in normal direction solved from the background field and $u_{n \text {,scat }}$ is the normal velocity due to the scattering of the sound waves on the frame of the porous material. 
Note that both the solution for the background sound field and for the scattered sound field satisfy the Helmholtz equation and are linear. Therefore, the solutions can be summed to find the solution for the total sound field.

\subsubsection{Tube resonators}

To validate the modelling approach for one-dimensional incident plane waves, a sample with 37 parallel tube resonators has been designed. Each tube resonator has a radius of $r_{t}=3 \mathrm{~mm}$ and the length of the resonators is optimised for maximal sound absorption between $850 \mathrm{~Hz}$ and $2500 \mathrm{~Hz}$ [14]. The optimisation is based on the work described in [31]. The analytical relations to determine the surface impedance and sound absorption coefficient based on the LRF approach are given in Appendix B. The design and dimensions of the sample are discussed in Appendix B3 and the numerical model of the sample itself is shown in Figure B.1. The developed model of this sample is shown in Figure 2.4. The closed end of tube resonators is tapered, since the sample used for the validation of the model is made from a solid aluminium cylinder in which the resonators are manufactured using a drill with a top-angle of $118^{\circ}$.

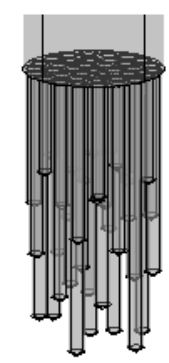

Figure 2.4: Numerical model of a sample with 37 tube resonators with radius $r_{t}=3 \mathrm{~mm}$ inside an impedance tube with radius $r_{\text {imp }}=25 \mathrm{~mm}$.

The measured sound absorption coefficient, the sound absorption coefficient estimated using the analytical LRF relations (Appendix B) and the predicted sound absorption coefficient using the hybrid modelling approach are shown in Figure 2.5. The predicted sound absorption coefficient shows excellent agreement with the measured sound absorption coefficient. Differences between the analytical results and the predicted and measured sound absorption coefficient are larger. It is assumed these are caused by the inlet effects of the tube resonators and the coupling effects between the tube resonators of a similar length. Similar effects were found by [31]. The good agreement between the predicted results and the measured results indicates that these effects are included directly in the hybrid model. 


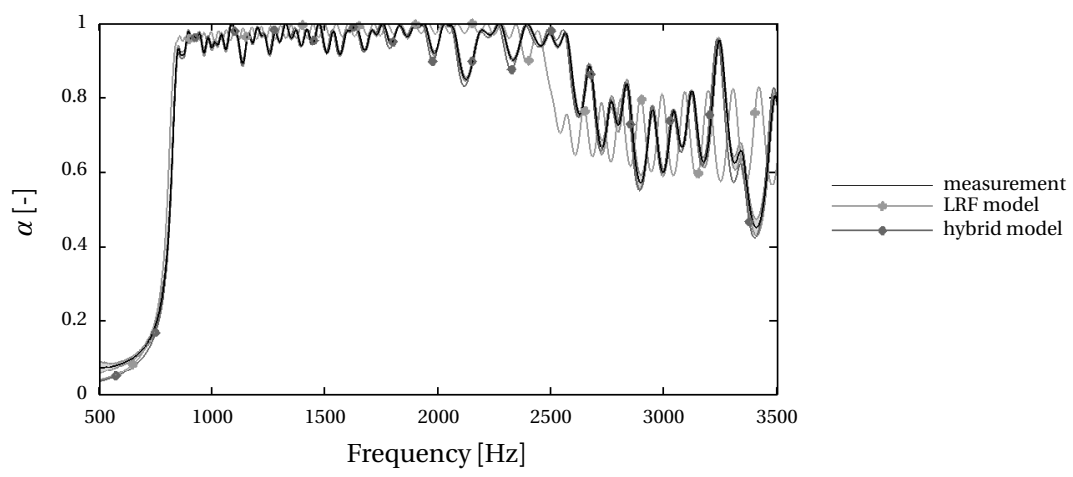

Figure 2.5: Sound absorption coefficient for an aluminum sample with 37 tube resonators with $r_{t}=3 \mathrm{~mm}$. The standard deviation of \pm 1 for the measured sound absorption coefficient is indicated with a shaded area.

\subsubsection{Stacked spheres in impedance tube}

The developed modelling approach is also validated for several structures of stacked marbles, using the impedance tube technique. Different amounts of glass marbles with a diameter of $6 \mathrm{~mm}$ or $12 \mathrm{~mm}$ were stacked in layers inside an impedance tube with a diameter of $50 \mathrm{~mm}$. An example of a stacking with 10 layers of spheres with a diameter of $D_{s}=6 \mathrm{~mm}$ is shown in Figure 2.6. The sound absorption coefficient for several of these structures was compared with the predicted sound absorption coefficient using the hybrid modelling approach. The results show a very good correlation between the experimental and the simulation results, as is shown in Figure 2.7. The models and experimental validation for all structures is described in detail in Paper A in Part II: Predicting sound absorption of stacked spheres: combining an analytical and numerical approach [15].

The behaviour of the sound absorption coefficient shown in Figure 2.7 resembles the behaviour of the sound absorption coefficient for tube resonators with one open and one closed end. These resonators have a peak in the sound absorption coefficient at the resonance frequencies $f_{n}$ :

$$
f_{n}=\frac{n c_{0}}{4 L}
$$

where $n=1,3,5, \ldots$ and $L$ is the length of the resonator. The peaks seen in Figure 2.7 indicate a dominant pore with a length greater than the layer height of the granular structure. This is expected, since the pores between the spheres or marbles are curved and therefore, the pores are longer than the height of the structure.

The properties of the viscous air inside the air-filled pores of the modelled sphere structure are based on the properties of a cylindrical tube using the LRF model, as described in Section 1.2.5. The radius of this characteristic tube is based on the ratio between the surface and volume of the spheres in a repetitive unit of the 


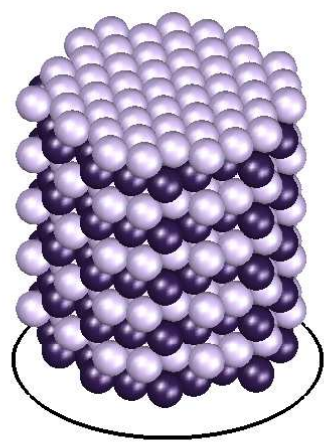

Figure 2.6: Geometry with 10 layers of stacked spheres with $D_{\mathrm{s}}=6 \mathrm{~mm}$ inside an impedance tube with $r_{\text {imp }}=25 \mathrm{~mm}$.

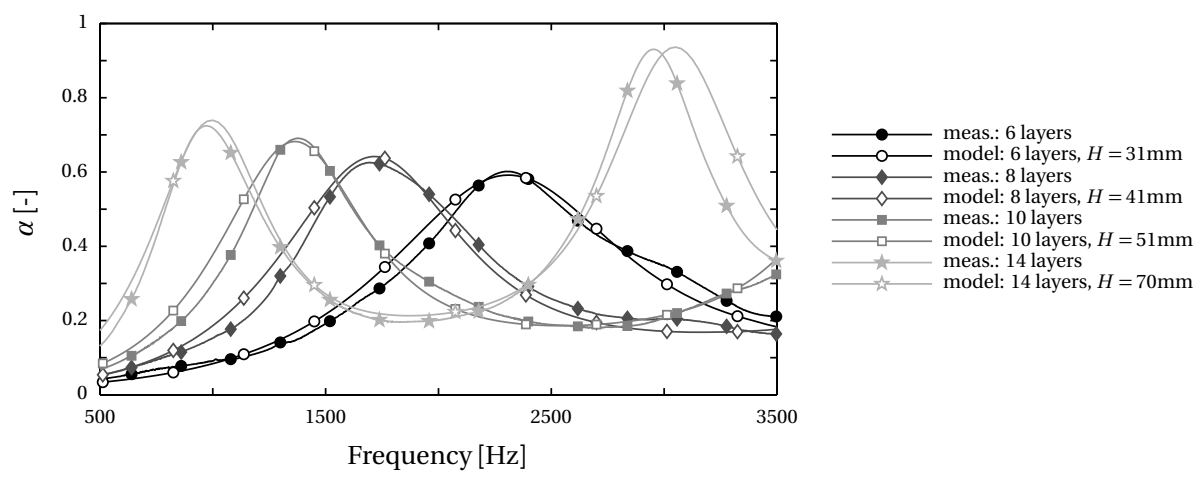

Figure 2.7: Validation of modelling approach for several structures of stacked spheres with $D=6 \mathrm{~mm}$, using the impedance tube technique. The measured absorption coefficient is indicated by the colored markers and the predicted absorption coefficient by the open markers [15].

sphere structure, the so-called hydraulic radius. The length of this characteristic pore and the porosity of the structure follow directly from the finite element model of the sphere structure and are not required when deriving the properties of the viscous air layer using the LRF model. A sensitivity study to determine the influence of the hydraulic radius on the sound absorption coefficient is included in Paper A, [15].

\subsection{Three-dimensional oblique incident waves}

The modelling approach for three-dimensional incident waves is based on the model for one-dimensional plane waves, with some small adjustments to ensure that the scattering sound field is local, such that the numerical model remains 
(relatively) small. The modelling approach for three-dimensional incident waves is validated for a box filled with stacked marbles for normal and oblique incidence using a novel measurement technique [37, 38]. The approach, model implementation, measurement technique and validation are discussed in detail in Paper B: Prediction of sound absorption of stacked granular materials for normal and oblique incident sound waves, [19].

\subsubsection{Hybrid modelling approach}

As done for the normal incident plane waves, the total sound field is solved by dividing the problem into two subsystems, as shown in Figure 2.1. The sound pressure for three-dimensional waves is given by:

$$
p_{j}=A_{j} e^{i k_{j} \xi(\mathbf{r}, \theta, \varphi)}+B_{j} e^{-i k_{j} \xi(\mathbf{r}, \theta, \varphi)}
$$

where the index $j$ indicates the medium and $\xi$ is a spatial coordinate defined by:

$$
\xi_{j}\left(\mathbf{r}, \theta_{j}, \varphi_{j}\right)=x \sin \theta_{j} \cos \varphi_{j}+y \sin \theta_{j} \sin \varphi_{j}+z \cos \theta_{j}
$$

where $\mathbf{r}=\{x, y, z\}$ are the coordinates, $\theta_{j}$ is the polar angle of incidence and $\varphi_{j}$ is azimuthal angle of incidence (both for medium $j$ ), as shown in Figure 2.8.

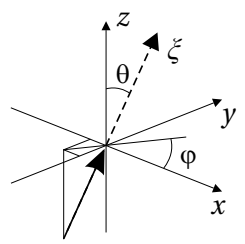

Figure 2.8: Conventions of direction, polar angle $\theta$ and azimuthal angle $\varphi$ for a wave in the direction along $\xi$.

The background sound field and the scattered sound field are implemented in the same numerical model to solve the total sound field. The background sound field is implemented according to Equation 2.7 using unit amplitude for all waves $\left(A_{\mathrm{I}}=B_{\mathrm{I}}=A_{\mathrm{II}}=B_{\mathrm{II}}=1\right)$ and the boundary conditions shown in Figure 2.9a. The scattered sound field is implemented using the boundary conditions shown in Figure 2.9b. A more detailed discussion of the implementation is given in Paper B, [19]).

\subsubsection{Validation for stacked spheres}

Measurements have been performed to show that the sound absorption coefficient depends on the angle of incidence and to show that the developed modelling approach can be used to predict the angle-dependent sound absorption coefficient of complex structures. The chosen structure, a sound hard box filled with glass marbles inside a sound hard baffle, is a well-defined structure and therefore possible to model. The marbles in the box are stacked in layers according to 


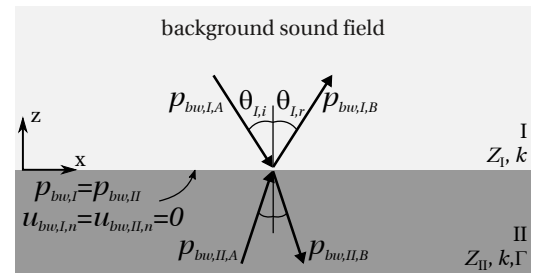

(a) Background sound field

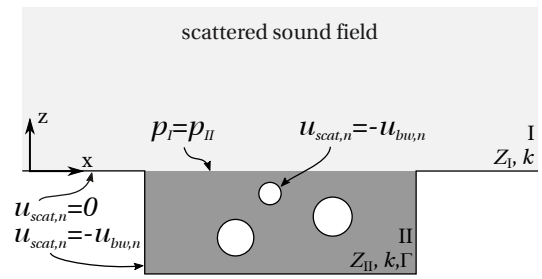

(b) Scattered sound field

Figure 2.9: Overview of implementation and boundary conditions in the threedimensional modelling approach.

the hexagonal close-packed (hcp) sphere packing. The box with marbles is shown in Figure 2.10a. The box was placed inside the anechoic room at the University of Twente, as shown in Figure 2.10b. Above the box with marbles, a speaker was placed to produce white noise. The sound absorption coefficient of the stacked marbles was measured using the so-called 8p-probe. This is a small cubic microphone array with eight pressure sensors; a novel measurement technique suitable for in-situ measurements, $[37,38]$. More details about the measurement setup, measurement technique and measurement uncertainties can be found in Paper $B$ in Part II, [19].

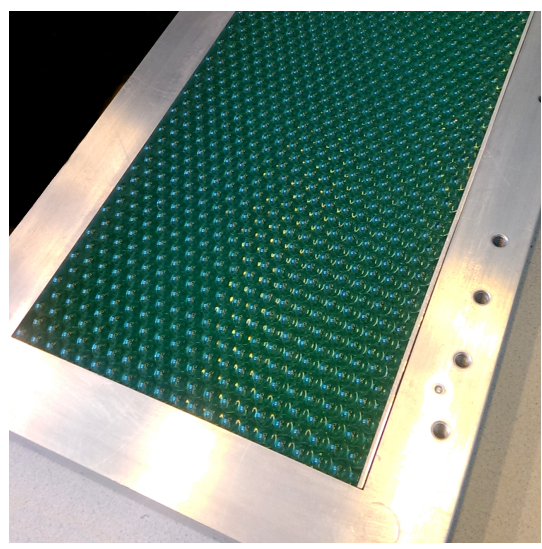

(a) Box with marbles.

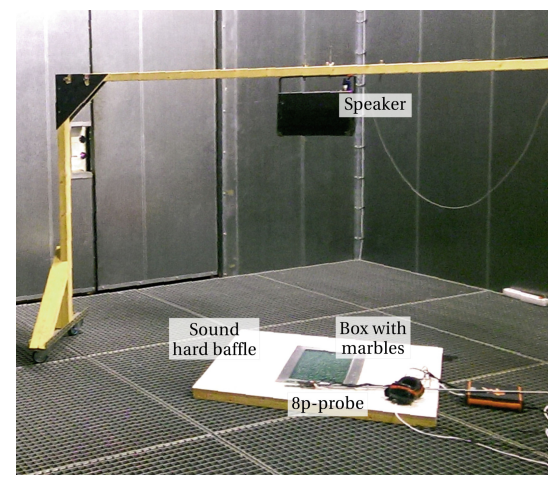

(b) Measurement setup.

Figure 2.10

Measurements for various angles of incidence have been performed. The measured and predicted sound absorption coefficient show a reasonable agreement, especially considering the limitations of the measurement setup and technique. The results for $\theta=0^{\circ}, \theta=30^{\circ}$ and $\theta=60^{\circ}$ are shown in Figure 2.11, more results can be found in Paper B in Part II, [19].

Figure 2.12 shows the predicted sound absorption coefficient for different angles of incidence. These curves show that the sound absorption coefficient indeed de- 

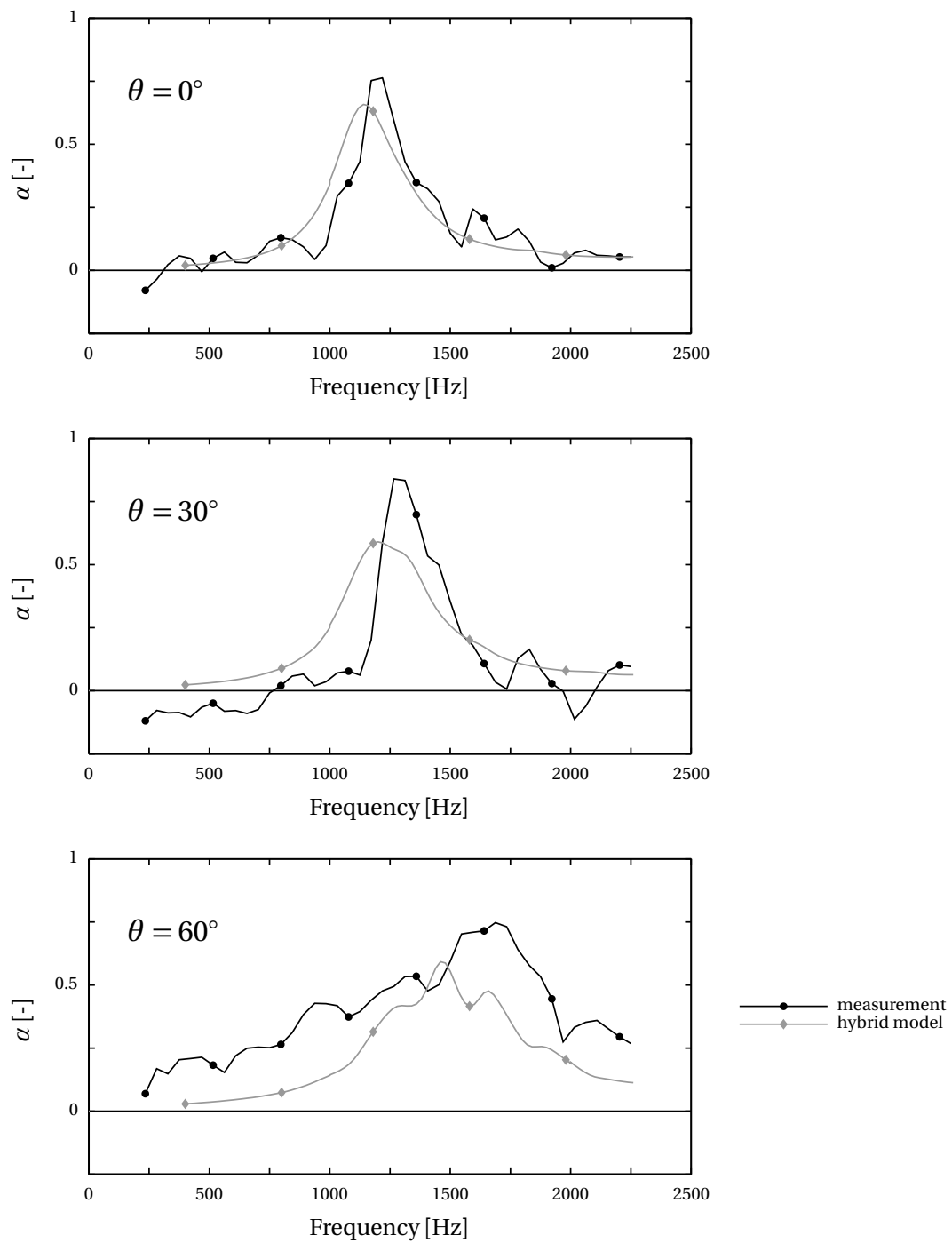

Figure 2.1 1: Predicted and measured sound absorption coefficient of stacked glass marbles inside a sound hard box for oblique incidence, measured with the 8p-probe.

pends on the angle of incidence. For a larger angle of incidence, the peak in the sound absorption shifts to higher frequencies. Assuming that the dominant pores in the structure of stacked marbles are directed along the $\mathrm{z}$-direction and behave as a tube resonator, the first resonance frequency depends on the length of this 
dominant pore. Decomposing the wave number $k$ yields:

$$
\begin{aligned}
& k_{x}=k \sin \theta_{j} \cos \varphi_{j} \\
& k_{y}=k \sin \theta_{j} \sin \varphi_{j} \\
& k_{z}=k \cos \theta_{j}
\end{aligned}
$$

where $k^{2}=k_{x}^{2}+k_{y}^{2}+k_{z}^{2}$. For normal incidence, $\theta=0^{\circ}$ and $\varphi=0^{\circ}$, the wave number in z-direction corresponds with the first resonance frequency: $2 \pi f_{1} / c_{0}=k_{1}=k_{z}$. The dimensions of this dominant pore and thus the corresponding resonance wave number in z-direction do not change when the angle of incidence increases. Therefore, the wave number $k_{1}$ and the first resonance frequency will increase with increasing angle of incidence.

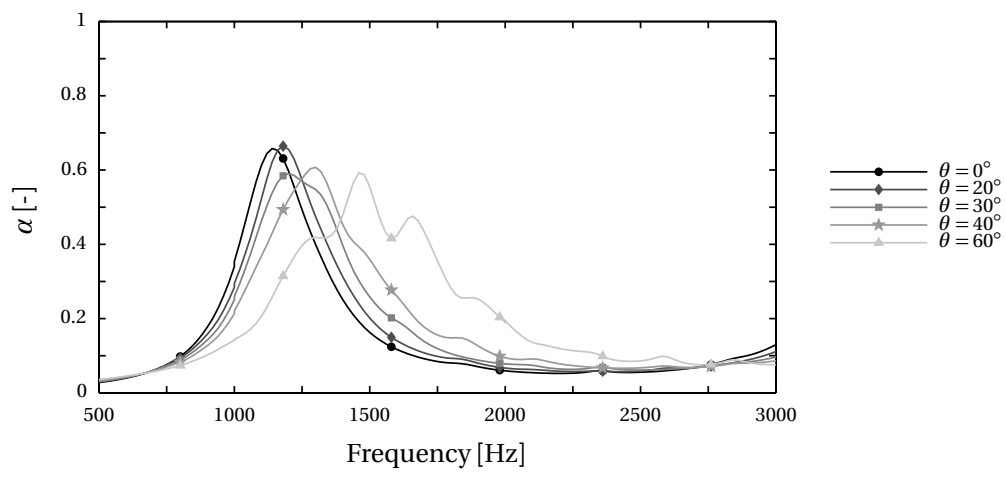

Figure 2.12: Predicted sound absorption coefficient for various angles of incidence.

\subsection{Design tool for porous road surfaces}

The developed modelling approach is used as a tool to design new porous asphalt road surfaces to reduce tyre/road noise. Within the project SVW ('Silent and Safe Road Traffic'), two prototype roads have been developed with a high sound absorption coefficient in the frequency range where tyre/road noise is most dominant. These two prototype roads are manufactured and tested on sound absorption, sound reduction and wet grip. The design process and the measured acoustical performance of these two road surfaces in combination with three different tyres has been described in Paper C: Integral approach to tire/road noise: considering both tire and road design, [17]. This article also includes a discussion of the measurements of the other tracks at the test area.

\subsubsection{Predictive model}

The hybrid modelling approach is used to investigate the influence of several road design parameters on the sound absorption coefficient. Since the developed modelling approach can also be used to find the sound absorption coefficient for 


\begin{tabular}{lccccc}
\hline & & $D_{s}$ & $H$ & $\Omega$ & $\theta$ \\
\hline reference model & & $6 \mathrm{~mm}$ & $25 \mathrm{~mm}$ & $20 \%$ & $0^{\circ}$ \\
\hline porosity of structure & Figure 2.16 & & & & \\
variation 1 & & $6 \mathrm{~mm}$ & $24 \mathrm{~mm}$ & $13 \%$ & $0^{\circ}$ \\
variation 2 & & $6 \mathrm{~mm}$ & $25 \mathrm{~mm}$ & $15 \%$ & $0^{\circ}$ \\
\hline layer thickness & Figure 2.17 & & & & \\
variation 1 & & $6 \mathrm{~mm}$ & $39 \mathrm{~mm}$ & $20 \%$ & $0^{\circ}$ \\
\hline stone size & Figure 2.18 & & & & \\
variation 1 & & $9 \mathrm{~mm}$ & $30 \mathrm{~mm}$ & $20 \%$ & $0^{\circ}$ \\
variation 2 & & $12 \mathrm{~mm}$ & $29 \mathrm{~mm}$ & $20 \%$ & $0^{\circ}$ \\
\hline angle of incidence & Figure 2.19 & & & & \\
variation 1 & & $6 \mathrm{~mm}$ & $24 \mathrm{~mm}$ & $20 \%$ & $20^{\circ}$ \\
variation 2 & & $6 \mathrm{~mm}$ & $25 \mathrm{~mm}$ & $20 \%$ & $40^{\circ}$ \\
variation 3 & & $6 \mathrm{~mm}$ & $25 \mathrm{~mm}$ & $20 \%$ & $60^{\circ}$ \\
\hline
\end{tabular}

Table 2.1: Properties of reference model and model variations, where $D_{s}$ is the stone size, $H$ is the layer thickness, $\Omega$ is the porosity and $\theta$ the angle of incidence.

oblique incident waves, new road surfaces can be optimised for larger angles of incidence. This is useful, since noise radiation by traffic noise is generally a problem for oblique incidence, as indicated in Figure 1.3.

Note that the models based on the developed modelling approach are limited to structures of equally sized spheres in a regular packing, a simplification of the structure of the asphalt concrete. More complex structures can be easily be implemented, since the hybrid modelling approach works for all types of structures. Therefore, the geometry of a more complex structure resembling the porous road surface, can be modelled in the numerical model, for example structures including the variation of stone size and shape.

It is assumed that the influence of some of the design parameters on the sound absorption coefficient can be predicted sufficiently, despite the simplification of the structure of the porous asphalt concrete to equally sized, stacked spheres. Multiple simulations have been performed to find the influence of the porosity, stone size and layer thickness on the sound absorption coefficient in comparison with a reference model for normal and oblique incidence. The properties of the reference model and model variations are listed in Table 2.1.

\section{Structure of pores and air pockets}

Using the hybrid modelling approach, only the air in between the stacked spheres is modelled in the numerical model, resulting in a structure of pores and air pockets. Two examples of these structures are shown in Figure 2.13. The spheres in these structures are modelled with a slight overlap $\delta$ to avoid meshing problems, 
as well as to control the porosity of the structures. These meshing problems and the influence of this overlap on the sound absorption is discussed more fully in Paper A.

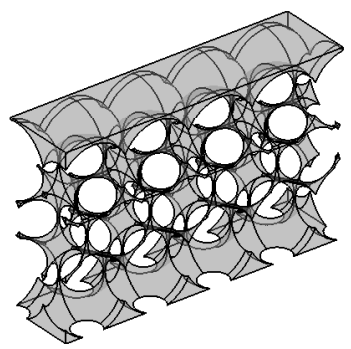

(a) $D_{s}=12 \mathrm{~mm}$

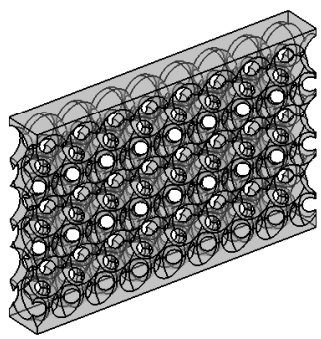

(b) $D_{s}=6 \mathrm{~mm}$

Figure 2.13: Structure of pores and air pockets in between the stacked spheres for spheres with diameter $D_{s}$. Note that only a small slice of the total structure is shown.

The diameter of the spheres determines the shape and dimensions of the pores and the air pockets. The influence of the shape and dimensions of this structure is investigated with simple models consisting of cones connected by small tubes, as illustrated in Figure 2.14. The results of these models are compared with a model with a single tube resonator of the same length and radius inside an impedance tube with radius $r_{\text {imp. }}$. It appears that the influence of the shape of the inlet (as illustrated in Figure 2.14a and 2.14b) results in only small changes in the amplitude and frequency of the peak in the sound absorption coefficient; the frequency increases and the amplitude decreases.

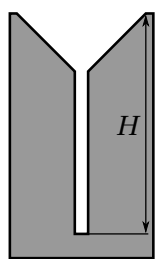

(a)

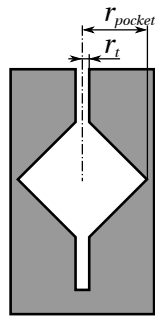

(b)

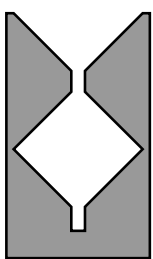

(c)

Figure 2.14: Illustration of the models used to determine the effect of the shape and dimensions of the air pores and pockets in between the spheres and the ratio between $r_{t}$ and $r_{\text {pocket }}$.

Changes in the total height of the structure and the number of cones affect both 
the frequency and the amplitude of the peak in sound absorption, as is illustrated in Figure 2.15. The frequency of the peak is determined mainly by the height of the structure, similar to the behaviour in the case of tube resonators. The amplitude depends both on the layer height of the structure and on the number of cones. An increase in height results in an increase in amplitude, while increasing the number of cones decreases the amplitude.

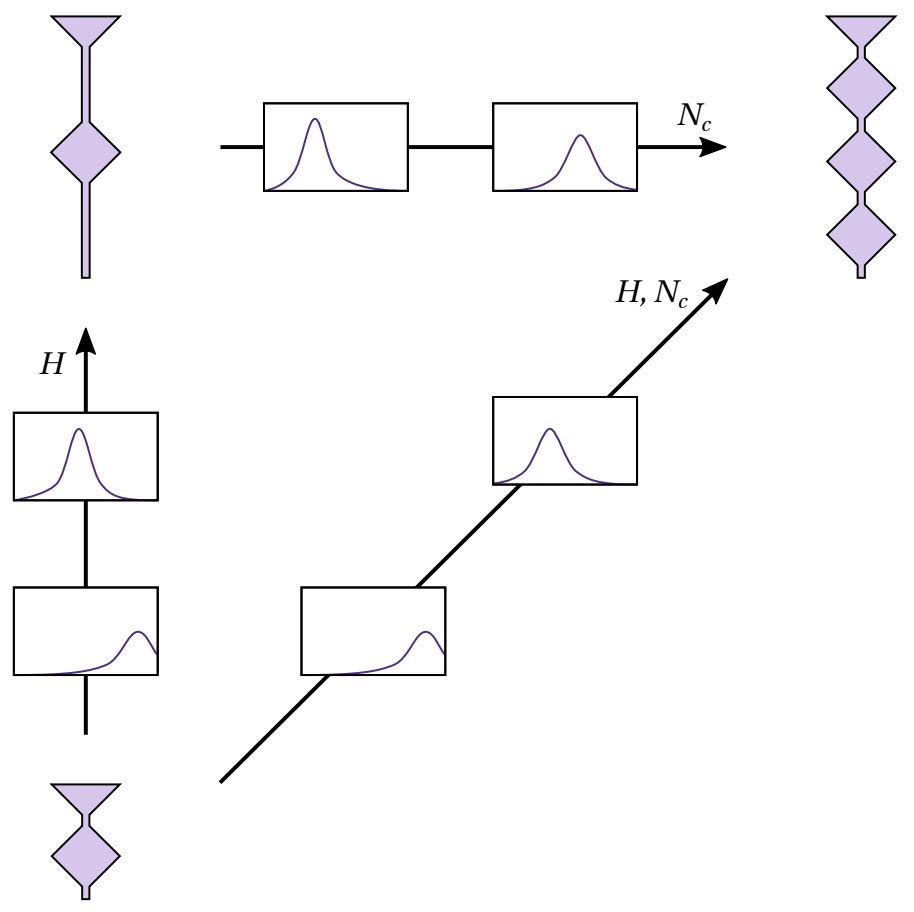

Figure 2.15: Influence of the height $H$ of the structure and the number of cones $N_{c}$ on the sound absorption coefficient.

A second parameter that influences the position of the peak in the sound absorption coefficient is the ratio $R_{\text {air }}=r_{t} / r_{\text {pocket }}$ between the radius of the tube $r_{t}$ and the radius of the air pocket $r_{\text {pocket }}$. A decrease in the ratio $R_{\text {air }}$ results in a decrease of the frequency of the peak.

Furthermore, the surface porosity $\Omega_{S}=r_{t}^{2} / r_{\text {imp }}^{2}$ of these structures influences the peak in the sound absorption coefficient. For tube resonators, the peak in the sound absorption is maximal at the optimal porosity, which is about $\Omega_{S}=2 \%$. For a smaller or larger surface porosity, the amplitude of the peak decreases. The surface porosity has little influence on the frequency of the peak. The same behaviour is seen with respect to these structures. 


\section{Porosity of sphere structure}

Figure 2.16 shows the influence of the porosity of a structure of stacked spheres, with the parameters listed in Table 2.1, on the sound absorption coefficient. A greater porosity shifts the peak in sound absorption to higher frequencies. Comparing this behaviour with that of tube resonators, a shift in the frequency of the peak in the absorption coefficient is associated with the tube length. This suggest that the length of the pores in the porous asphalt concrete increases for a greater porosity. It can also mean that the flow resistivity of the pores decreases, so the sound waves can travel further inside the pores.

Figure 2.16 also shows that the amplitude and the width of the peak depends on the porosity. The properties of the viscous air layer, medium II, depend on the socalled hydraulic radius of a characteristic tube representing the dominant pore in between the spheres. This radius is larger for structures with a higher porosity and the viscothermal properties become less dominant for tubes with a larger radius, according to the LRF model described in Section 1.2.5. Furthermore, a structure of tube resonators has a porosity for which the amplitude of the sound absorption coefficient is largest. For structures with a different porosity, the peak of the sound absorption coefficient becomes lower and wider.

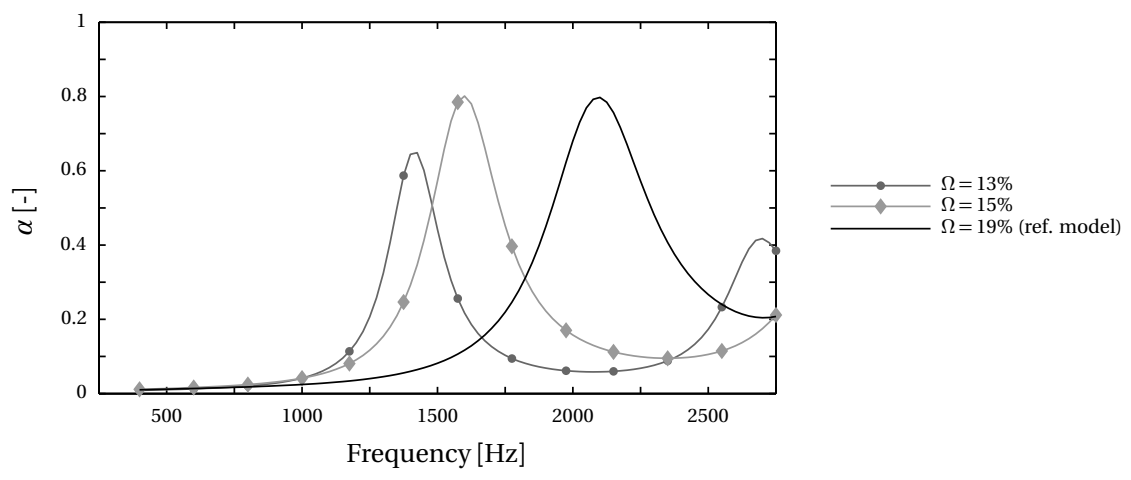

Figure 2.16: Influence of the porosity of the granular structure on the sound absorption coefficient. The solid black line shows the sound absorption coefficient of the reference model.

\section{Layer thickness}

The influence of the layer thickness of the structure is shown in Figure 2.17. The figure shows that a larger layer thickness corresponds with a lower frequency of the peak in the absorption coefficient, which is expected when comparing the behaviour with that of a tube resonator.

Figure 2.17 also shows a small decrease in the amplitude of the peak in the sound absorption coefficient. This can be explained by the combination of the increase 
in both the layer height and the increase in the number of air pockets, as described in Section 2.4.1.

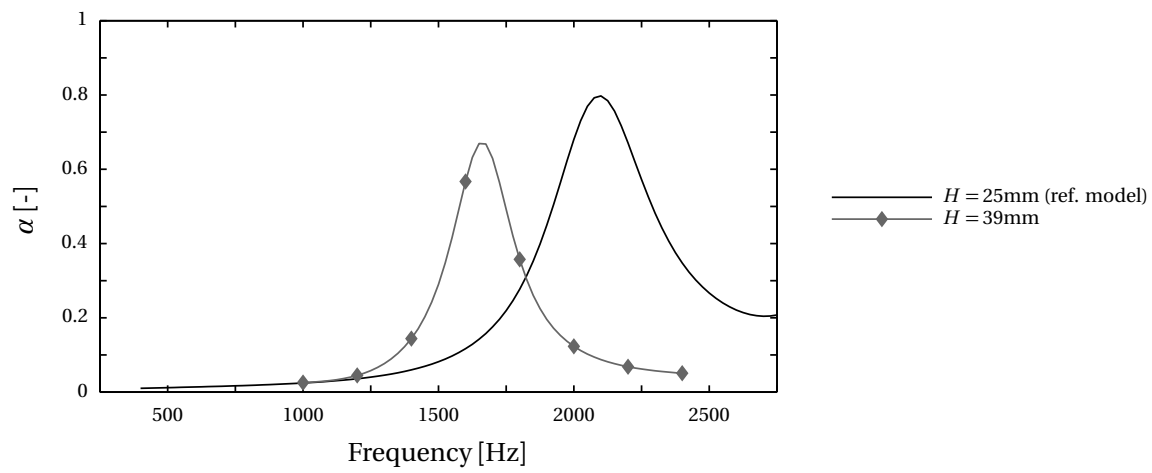

Figure 2.17: Influence of the layer thickness of the structure on the sound absorption coefficient. The solid black line shows the sound absorption coefficient of the reference model.

\section{Stone size}

The influence of the stone size on the sound absorption coefficient is shown in Figure 2.18. Two structures with larger spheres $\left(D_{s}=9 \mathrm{~mm}\right.$ and $\left.D_{s}=12 \mathrm{~mm}\right)$ are implemented in such a way that the layer thickness and the porosity are similar to those of the structure with smaller spheres $\left(D_{s}=6 \mathrm{~mm}\right)$.

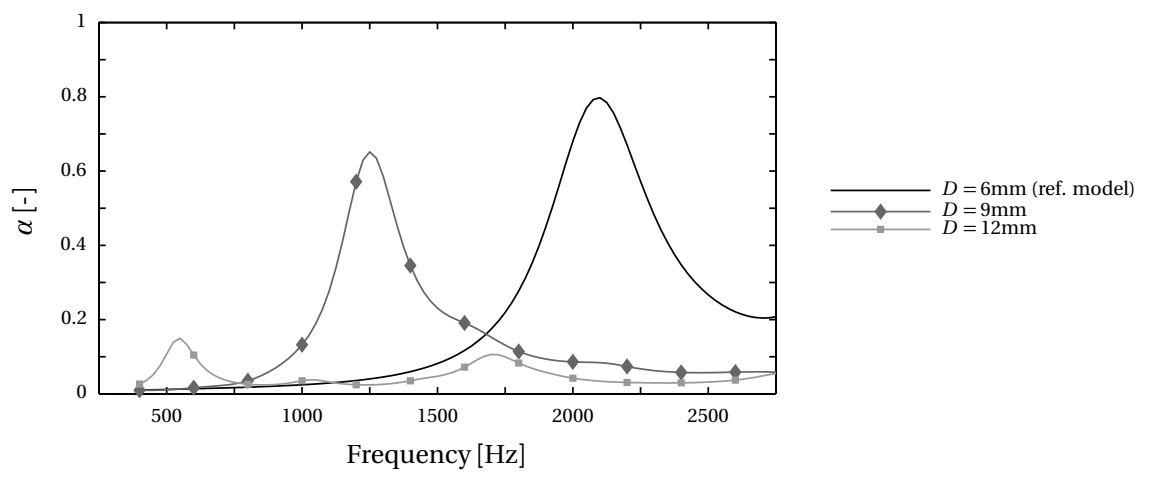

Figure 2.18: Influence of the stone size on the sound absorption coefficient. The solid black line shows the sound absorption coefficient of the reference model.

To maintain a similar porosity for each structure, the spheres are modelled in such a way that they are slightly overlapping. The larger the stone size, the more the spheres overlap. Therefore, the pores in between the spheres will have a smaller 
radius for the models with a larger stone size. Furthermore, for the models with the larger spheres, the pockets of air inside the structure are also larger. Therefore, the ratio $R_{\text {air }}$ between the radius of the pores and the radius of the air pockets is small for a structure with large stones, as can be seen in Figure 2.13.

Figure 2.18, shows that the amplitude of the peak of the sound absorption coefficient is smaller for the models with a larger stone size. Also, the frequency of the peak decreases for an increasing stone size. This behaviour corresponds with the behaviour seen for the simple models of cone shaped air pockets connected with tubes in Section 2.4.1. This indicates that the sound absorption is smaller for less homogeneous structures, since the ratio $R_{\text {air }}$ is smaller and thus less homogeneous for the models with a larger sphere size.

\section{Angle of incidence}

In Figure 2.19, the sound absorption coefficient for four different angles of the incidence is shown. The angle of incidence of the reference model is $\theta=0^{\circ}$. The models used for the other angles of incidence are equal to the reference model, but have a different direction of the background sound field.

Figure 2.19 shows that the behaviour of the sound absorption coefficient changes for oblique incidence. The amplitude of the absorption peak decreases and a second peak appears. It is assumed that when the angle of incidence increases, the sound waves follow a different path through the structure causing different behaviour.

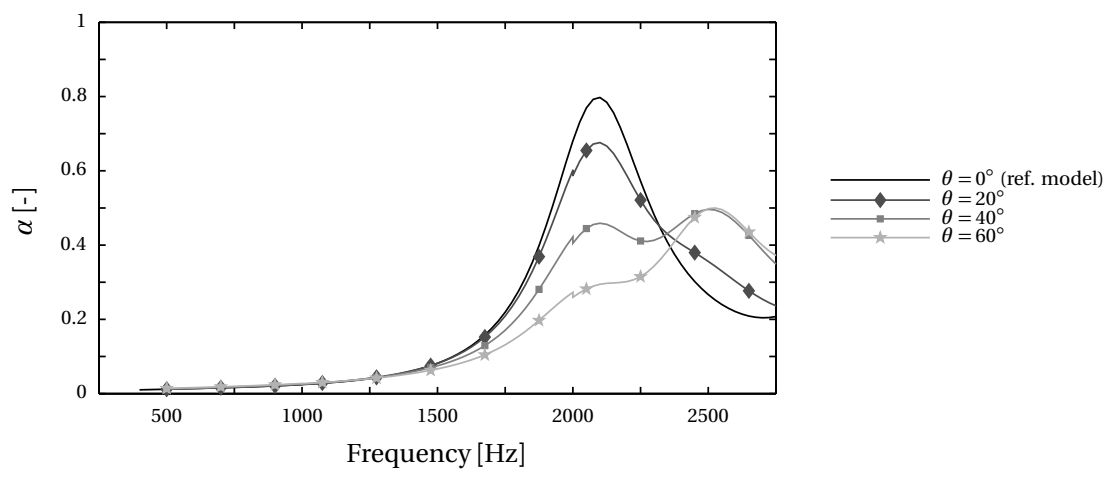

Figure 2.19: Influence of the angle of the incident sound waves on the sound absorption coefficient. The solid black line shows the sound absorption coefficient of the reference model.

\section{Influence on sound absorption coefficient}

The influence of the parameters on the sound absorption coefficient is summarised in Figure 2.20. It is found that: 
- the sound absorption coefficient is larger when the porosity of the structure is higher;

- the width of the peak in the absorption coefficient increases with an increasing porosity and a decreasing stone size;

- the amplitude of this peak depends on the porosity: it decreases for an increasing layer thickness and an increasing angle of incidence;

- the peak shifts to higher frequencies for an increasing porosity, decreasing stone size, decreasing layer thickness and an increasing angle of incidence.

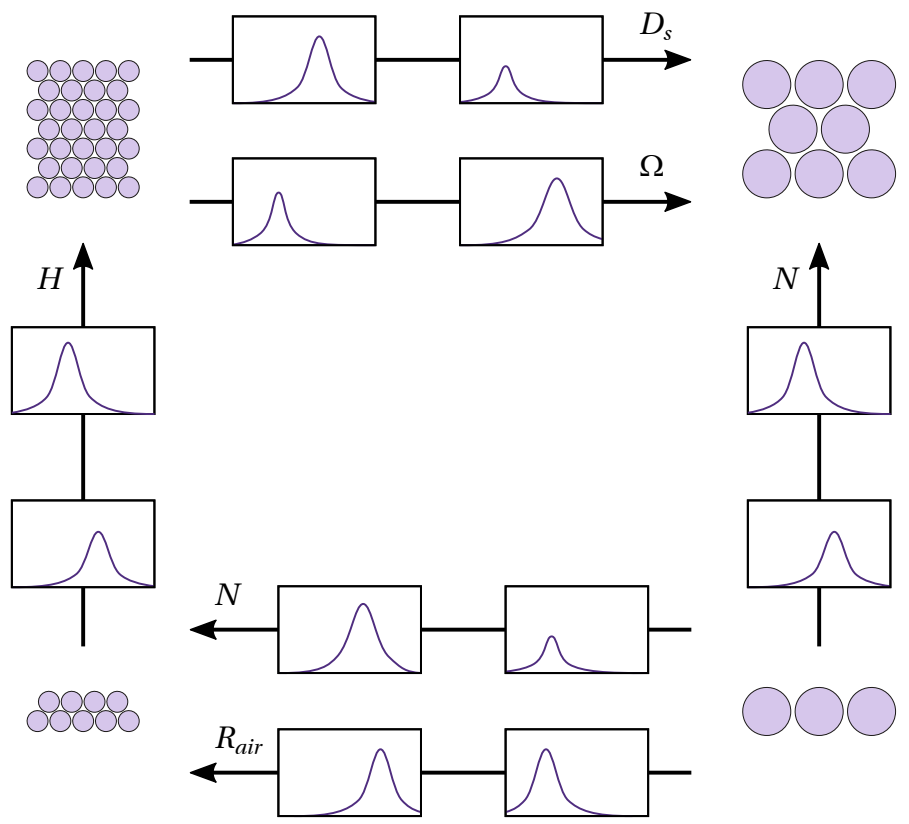

Figure 2.20: Influence of parameters on the sound absorption coefficient, where $H$ is the layer height, $\Omega$ is the porosity of the structure, $R_{\text {air }}=r_{t} / r_{\text {pocket }}$ is the ratio between the pores and the air pockets, $N$ the number of layers of spheres in the structure and $D_{s}$ the diameter of the spheres.

\subsubsection{Prototype roads}

The model is used as a design tool to find road parameters which should optimise the sound absorption coefficient of porous road surfaces for the reduction of tyre/road noise for oblique incidence. The optimisation is focused on the $1000 \mathrm{~Hz}$ octave band, since this octave band includes the most important frequencies in tyre/road noise. As a design tool, the model results only give guidelines, since no model has been made with a structure exactly resembling a potential asphalt mixture. The model results are combined with design criteria based on measure- 
ments and criteria found in the literature. This resulted in the following road design criteria:

- a high porosity;

- small stone sizes;

- a substantial layer thickness;

- a smooth road surface.

Furthermore, some construction constraints had to be taken into account: (1) the porosity of the road surface is limited to $25 \%$ and (2) the layer thickness of a single layer of asphalt concrete is limited, depending on the porosity and stone size. Therefore, the choice was made to develop and manufacture two prototype roads:

- a single layered road with the maximum layer thickness;

- a double layered road to achieve a greater layer thickness.

Finally, the stone sizes (and material) used for these prototype roads depend on the availability of the material and the requirements for (wet) grip, determined by other participants in the SVW project.

The first prototype road surface is a single layered porous asphalt concrete, called OPA 6 (track 8), with stone sizes ranging from $2 \mathrm{~mm}$ to $6 \mathrm{~mm}$ and porosity of about $25 \%$. The other road surface is a double layered porous asphalt concrete, called Twinlay (track 7 ), with stone sizes between $2 \mathrm{~mm}$ and $4 \mathrm{~mm}$ and a similar high porosity as track 8 . The properties of these test tracks are listed in Appendix C under track 7 and 8.

Various measurements have been performed at the test tracks to determine the sound absorption coefficient and the sound radiation close to the tyre (at CPX positions) and at the side of the road (during pass by measurements). The performance of all the tyre and road combinations is discussed in detail in Paper C, [17]. Here, the results relevant to the design process are summarized.

\section{Sound absorption coefficient}

The sound absorption coefficient of the test tracks for normal incidence is measured in-situ with the impedance tube technique. The tube was placed perpendicular to the road surface using a flange, as shown in Figure 2.21.

For each road surface, the sound absorption coefficient was measured in ten equally spaced locations in longitudinal direction of the test track. The results for the two prototype tracks and track 6 , a reference road surface, are given in Figure 2.22. The sound absorption coefficient of tracks 7 and 8 for the octave bands are listed in Table 2.2. Both prototype tracks have a high sound absorption coefficient. The results for track 7 show that the sound absorption coefficient in the $500 \mathrm{~Hz}$ octave band is slightly larger, which can be explained by the greater layer thickness of this track. 


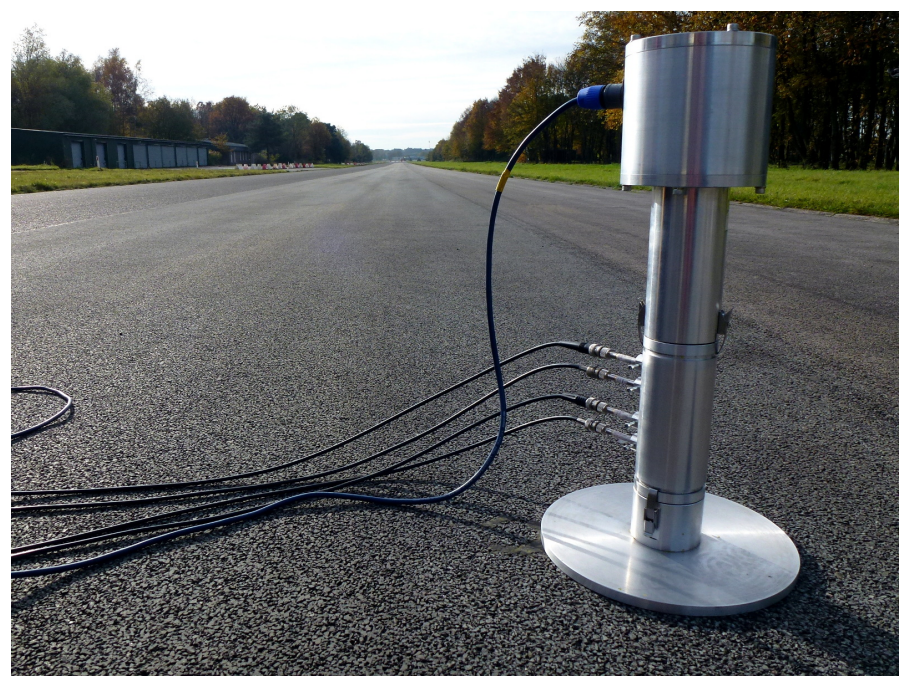

Figure 2.21: Impedance tube with flange used for in-situ sound absorption measurements at Twente Airport.

\begin{tabular}{lcccccccc}
\hline \multicolumn{2}{c}{ Track 6: ISO/R117 } & \multicolumn{3}{c}{ Track 7: Twinlay } & \multicolumn{3}{c}{ Track 8: OPA 6 } \\
& $\alpha$ & S.D. & & $\alpha$ & S.D. & & $\alpha$ & S.D. \\
$500 \mathrm{~Hz}$ & 0.03 & 0.01 & $500 \mathrm{~Hz}$ & 0.59 & 0.08 & $500 \mathrm{~Hz}$ & 0.55 & 0.07 \\
$1000 \mathrm{~Hz}$ & 0.03 & 0.01 & $1000 \mathrm{~Hz}$ & 0.48 & 0.04 & $1000 \mathrm{~Hz}$ & 0.56 & 0.04 \\
$2000 \mathrm{~Hz}$ & 0.06 & 0.01 & $2000 \mathrm{~Hz}$ & 0.47 & 0.04 & $2000 \mathrm{~Hz}$ & 0.49 & 0.04 \\
\hline
\end{tabular}

Table 2.2: Sound absorption coefficient $\alpha$ and the standard deviation S.D. in octave bands for track 6,7 and 8 .

Since the prototype tracks were optimised for the reduction of tyre/road noise for oblique incidence, the sound absorption coefficient for oblique incidence has also been measured. These measurements were performed in an anechoic room at the University of Twente using small cylindrical slabs. The slabs were taken from the test tracks using a 300mm bore. The slabs contain the road surface and a part of the (sound hard) fundament of the road. The sound absorption coefficient for oblique incidence is measured with a small cubic microphone array, the 8p-probe, $[37,38]$. The measurement technique and measurement uncertainties are discussed in more detail in Paper B, [19]. The results are shown in Figure 2.23 and Figure 2.24. The results for normal incidence $\left(\theta=0^{\circ}\right)$ differ from the results when using the impedance tube technique, since with the 8p-probe the sound absorption of small slabs is measured using the entire sound field around the slabs. For the impedance tube technique, the sound absorption of the tracks is measured in situ, which causes leakage of the sound to the area around the tube. De- 


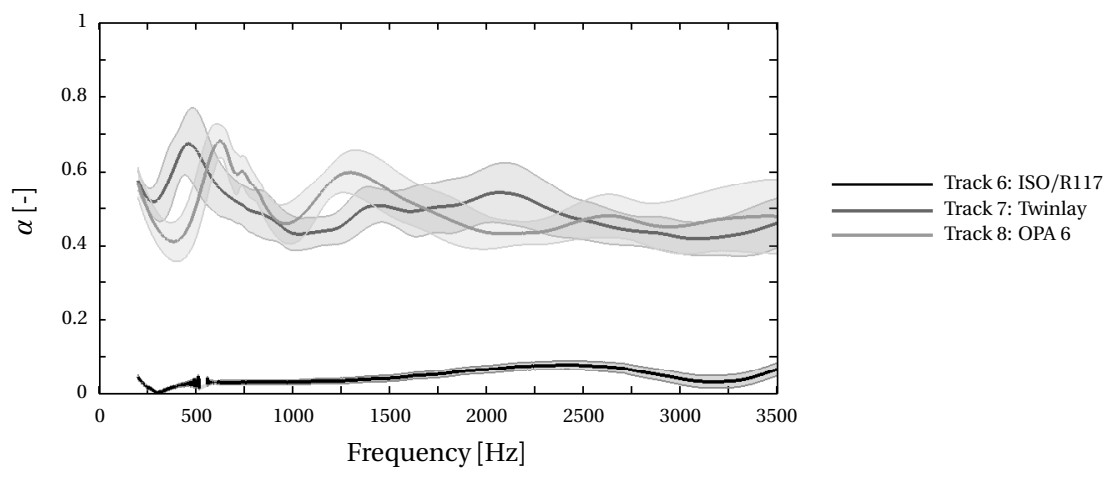

Figure 2.22: Measured sound absorption coefficient for normal incidence for the prototype tracks. The shaded area indicates a \pm 1 standard deviation.

spite these differences, the measurements show the influence of oblique incident sound waves on the sound absorption coefficient.

The results in Figure 2.23 and Figure 2.24 clearly show that the sound absorption coefficient depends both on the frequency and the angle of incidence. The measurement results show that the peak in the sound absorption coefficient shifts to higher frequencies for a larger angle of incidence. For $\theta=0^{\circ}$ and $\theta=30^{\circ}$, the maximum sound absorption coefficient is in the $1000 \mathrm{~Hz}$ octave band and for $\theta=60^{\circ}$ the maximum value is shifted to the $2000 \mathrm{~Hz}$ octave band.

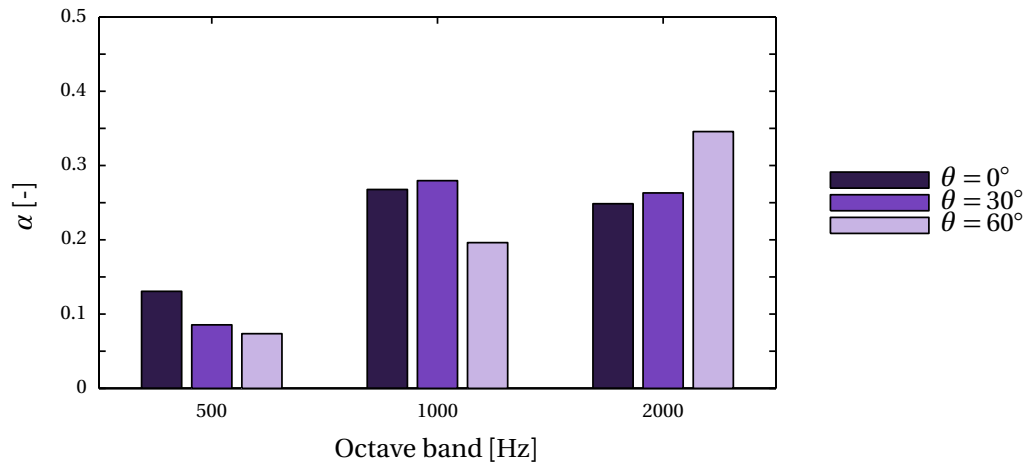

Figure 2.23: Sound absorption coefficient of prototype track 7 for oblique incidence measured with the 8 p-probe in the anechoic room. 


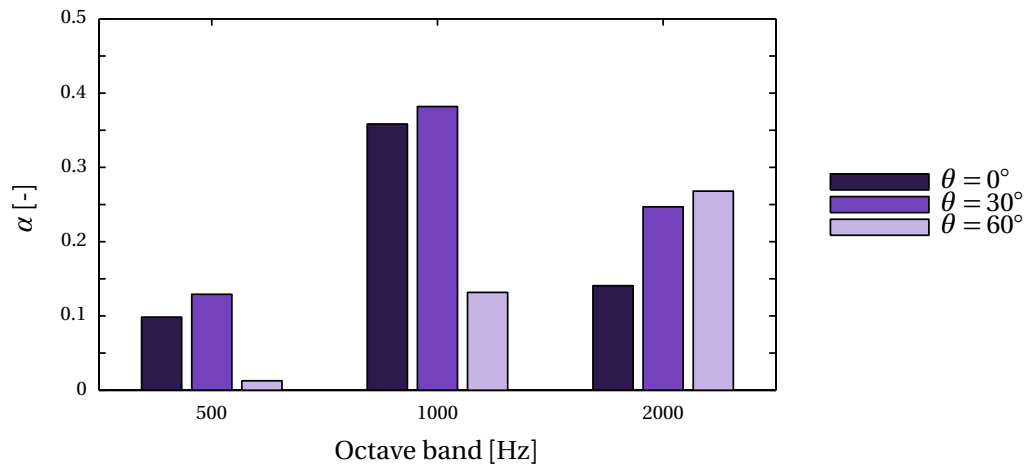

Figure 2.24: Sound absorption coefficient of prototype track 8 for oblique incidence measured with the $8 \mathrm{p}$-probe in the anechoic room.

\section{Sound radiation}

The sound radiation of the prototype road surfaces is measured with closeproximity (CPX) measurement and controlled pass by measurements. The measurement methods used are described in more detail in Paper C, [17]. The CPX measurement method yields accurate and repeatable results of the sound pressure level close to the tyre. The pass by measurements include the path between the sound source and the microphones, which is of interest when reducing the tyre/road noise for the receiver, as illustrated in Figure 1.3. However, these measurements are more sensitive to disturbances such as wind noise.

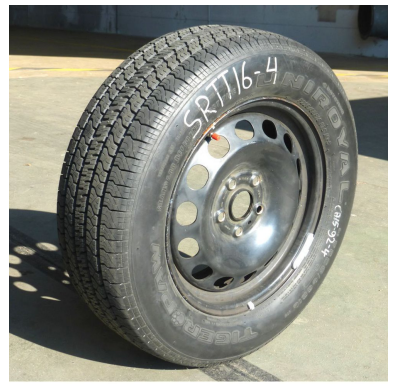

(a) SRTT Tigerpaw

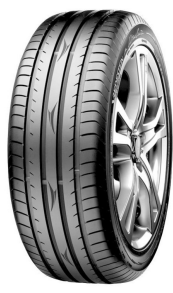

(b) Ultrac Cento XL

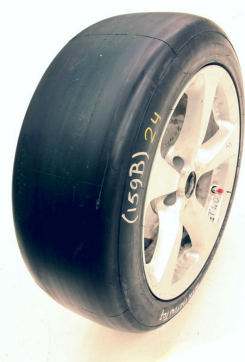

(c) Slick tyre

Figure 2.25: Photos of the three tyres used for the CPX measurements and the controlled pass by measurements.

The sound radiation for a car driving at different (constant) velocities and three different set of tyres have been measured. The results for the different velocities are compared using a velocity correction, according to [49, Ch. 9] and Paper C, [17]. The tyres are shown in Figure 2.25. The results are expressed in sound reduction $\Delta$ SPL, comparing the measured sound pressure level of each tyre and road 
combination with the sound pressure level of a standard reference tyre (SRTT) on the standard reference road surface (ISO/R117, track 6):

$$
\Delta \mathrm{SPL}=\mathrm{rms}_{\mathrm{SRTT}, 6}-\mathrm{rms}_{i_{\mathrm{tyre}}, k_{\text {track }}}
$$

where $\mathrm{rms}_{\mathrm{SRTT}, 6}$ is the root mean square (rms) value of the sound pressure level $(\mathrm{SPL})$ of the reference tyre on the reference road surface and $\mathrm{rms}_{i_{\mathrm{tyre}}, k_{\text {track }}}$ the rms value of the SPL for tyre $i_{\text {tyre }}$ and track $k_{\text {track }}$.

Figure 2.26 shows the sound reduction measured at the CPX positions, close to the tyre and road. The sound reduction depends both on the tyre and on the road surface. The sound reduction of track 6 is much lower than for the two prototype tracks, which is expected, since the sound absorption coefficient of track 6 is very low (Figure 2.22). The sound reduction of test track 7 is greater for most combinations than for that of track 8 .

Figure 2.27 shows that the sound reduction for the controlled pass by measurements, measured at $7.5 \mathrm{~m}$ from the centre of the road and at a height of $h=3.0 \mathrm{~m}$. The repeatability of these measurements for the $500 \mathrm{~Hz}$ octave band is lower than for the other octave bands and the CPX measurements, as indicated by the errorbar representing the standard deviation. For the $500 \mathrm{~Hz}$ octave band, the results for the different test tracks show little variation, though the sound reduction of track 8 is lowest.

The results for the $1000 \mathrm{~Hz}$ octave band show a large sound reduction for tracks 7 and 8 for all the tyres. The variation in performance for the slick tyre on the different road surfaces is much smaller than for the other tyres. The results in the $2000 \mathrm{~Hz}$ octave band show little variation in sound reduction for the different tyres. However, the sound reduction for tracks 7 and 8 is much larger than for track 6 .
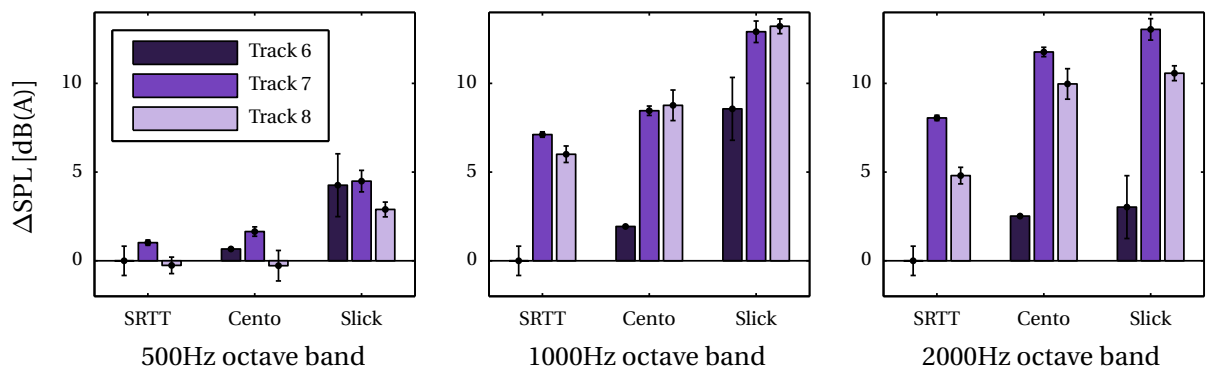

Figure 2.26: Sound reduction per road for CPX measurements, compared to SRTT on track 6 , ISO/R117. The error bar indicates $\mathrm{a} \pm 1$ standard deviation.

\subsubsection{Discussion on design of prototype road surfaces}

Using the hybrid modelling approach as design tool it is expected that a higher porosity gives a larger sound absorption coefficient. This is confirmed when considering the sound absorption coefficient and porosity of all the test tracks, as is 

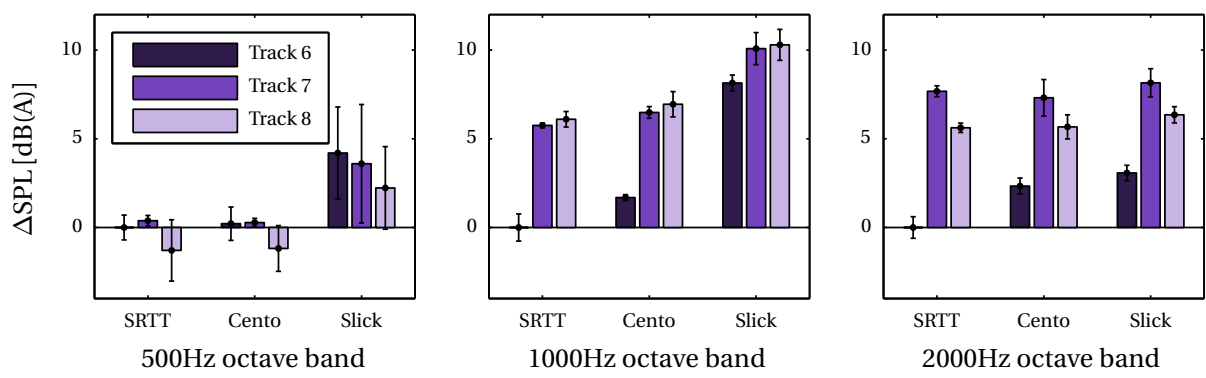

Figure 2.27: Sound reduction for the reference track (track 6 ) and the prototype tracks 7 and 8 . Measured with controlled pass by measurements (at $7.5 \mathrm{~m}$ from the centre of the road and at $h=3.0 \mathrm{~m}$ ), compared to SRTT on track 6, ISO/R117. The error bar indicates a \pm 1 standard deviation.

done in Paper C, [17]. The influence of the stone size is more difficult to confirm with the performed measurements.

The prototype road surfaces were designed such that the sound absorption coefficient for normal and oblique incidence was large in the $1000 \mathrm{~Hz}$ octave band, the frequency range important for tyre/road noise. For normal incidence, the sound absorption coefficient is listed in Table 2.2 and is large for all octave bands. The results for oblique incidence (in Figure 2.23 and Figure 2.24) show that the absorption coefficient in the $1000 \mathrm{~Hz}$ octave band is large for $\theta=0^{\circ}$ and $\theta=30^{\circ}$. The largest sound absorption coefficient for $\theta=60^{\circ}$ is found in the $2000 \mathrm{~Hz} \mathrm{oc}$ tave band. This indicates that the peak in amplitude seems to shift to higher frequencies for a larger angle of incidence, as was predicted by the simulations (Figure 2.19).

The CPX and controlled pass by results show that the sound reduction of the two prototype tracks is large in the $1000 \mathrm{~Hz}$ and $2000 \mathrm{~Hz}$ octave band, up to $13 \mathrm{~dB}(\mathrm{~A})$ for the prototype tracks in the $1000 \mathrm{~Hz}$ band. This behaviour is expected, since the sound absorption coefficient in these octave bands is also large. The sound reduction in the $500 \mathrm{~Hz}$ band was expected higher, since the sound absorption coefficient (measured with the impedance tube) in this octave band was also large. However, the wavelengths in the $500 \mathrm{~Hz}$ octave band are much larger than the layer thickness of this track. Track 7, the track with the greatest layer thickness, has the best sound reduction in the $500 \mathrm{~Hz}$ octave band.

Furthermore, the CPX and controlled pass by results show that the sound reduction depends on both the tyre and the road. This is discussed in more detail in Paper C, [17], in which the influence of some tyre and road parameters on the sound radiation are considered. 


\subsection{Other applications}

The hybrid analytical/numerical modelling approach has been developed to predict the sound absorption of porous asphalt concrete to improve models that predict tyre/road noise and to improve porous road surfaces in the design phase. However, the developed modelling approach is not limited to these applications.

A different application of this modelling approach is discussed in Paper D: Modelling approach for 3D printed sound absorbing panels, [18]. Here, the focus is on developing a modelling approach to predict the sound absorption coefficient of 3D printed sound reducing panels for broadband sound absorption. These panels will be manufactured using Selective Laser Sintering (SLS), a 3D printing technique. The research is focused on the influence of the additional sound absorbing material properties on the sound absorption coefficient of the structures. Therefore, the modelling approach is extended with sound absorbing material properties and is validated for a structure with (thin-walled) coupled tube resonators manufactured with SLS in combination with the material Polyamide (PA12).

\subsubsection{Modelling approach extended with impedance of material}

The total solution for the combined sound field can be solved directly for problems with normal incident plane waves and assuming plane wave propagation. The background sound field is implemented directly in the numerical model using an incident wave with unit amplitude. The porous or granular structure is also included in this numerical model. The properties of medium II are determined analytically using the LRF model. Solving this model directly gives the solution for the total combined sound field, which is equal to the solution for the total sound field using the complete hybrid modelling approach. Two advantages of this approach are that:

- the boundaries of the porous or granular structure can be changed from sound hard to sound absorbing by applying an impedance to the boundaries of the structure,

- when modelling a structure of tube resonators, the viscous properties of the air inside the tube resonators can be calculated for all resonators separately, since the background sound field is solved numerically.

The advantages of the hybrid modelling approach, such as reduced computational time and including inlet effects of the tube resonators and acoustic coupling of the tube resonators, are also valid for this simplified approach.

To investigate the influence of the material properties of panels manufactured with SLS, this simplified modelling approach is extended with impedance boundary conditions. The influence of the applied impedance on the sound absorption coefficient is discussed in Paper $\mathrm{D}$ for a model with a single tube resonator.

\subsubsection{Validation for 3D printed sample}

This adjusted modelling approach with impedance boundary conditions is validated for a 3D printed structure with coupled tube resonators, as discussed in 
Paper D, [18]. The structure with three coupled resonators is optimised for sound absorption between $f=1250 \mathrm{~Hz}$ and $f=2000 \mathrm{~Hz}$. The optimisation is based on the analytical relations for coupled tube resonators using the LRF theory as described by [56] and summarised in Appendix B. The design and dimensions of this structure are discussed in Appendix B4.

The optimised structure is manufactured with SLS and Polyamide 12 (with the powder PA 2200 as basis). A photo of the sample is shown in Figure 2.28.

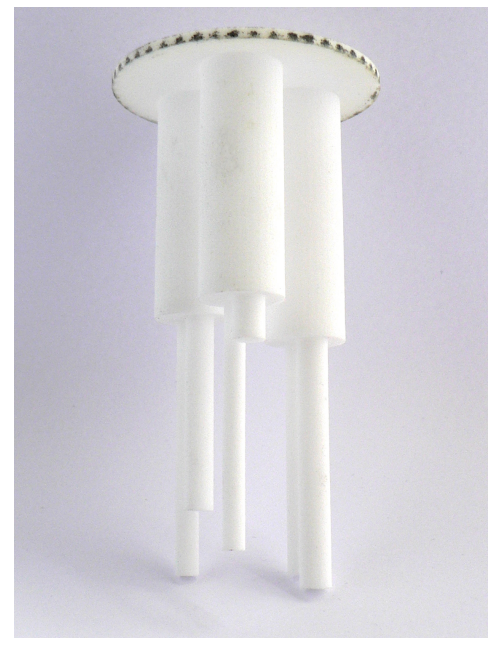

Figure 2.28: The designed sample with three coupled tube resonators.

The sound absorption coefficient for the structure with the coupled tube resonators is shown in Figure 2.29. The estimated sound absorption coefficient using the analytical LRF relations and the sound absorption coefficient predicted by a hybrid model with sound hard boundaries are very similar. Note that both the analytical LRF model and the hybrid modelling approach are validated for tube resonators made from sound hard materials. However, the measurement results show that the peaks in the sound absorption are shifted to the right compared to the analytical and numerical results, which suggests additional sound absorption by the material of the structure itself.

Figure 2.30 shows the predicted sound absorption coefficient for a numerical model with an impedance of:

$$
Z=\tau_{\operatorname{Re}}\left(\frac{f^{2}}{20.0^{2}}-\frac{f}{0.741}+18980\right)+\tau_{\operatorname{Im}}\left(\frac{f^{2}}{23.6^{2}}-\frac{f}{0.0907}+20610\right) i
$$

where $f=\omega / 2 \pi$ is the frequency and $\tau_{\mathrm{Re}}$ and $\tau_{\mathrm{Im}}$ are shape factors. The impedance $Z$ is based on measurements of the surface impedance of slivers of the printed material. The shape factor $\tau_{\mathrm{Re}}$ was set to 1 , changing the shape factor of the real part of the impedance only affects the amplitude of the sound absorption coefficient. For example, when using $\tau_{\mathrm{Re}}=15$ the amplitude of the peaks 


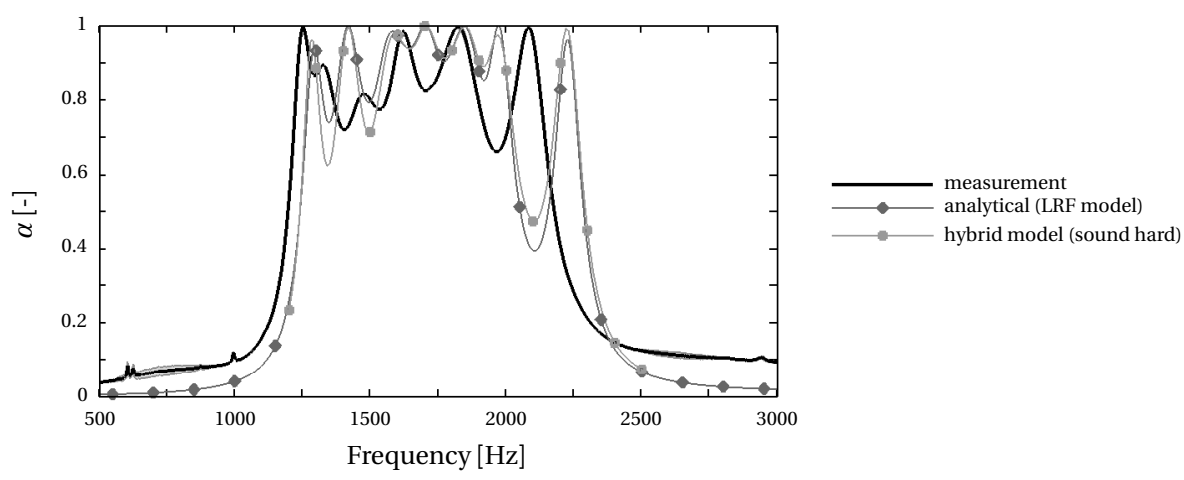

Figure 2.29: Measured and predicted sound absorption coefficient assuming sound hard boundaries for the structure with coupled tube resonators. The shaded area around the curve for the measured sound absorption coefficient indicates \pm 1 standard deviation

is larger and the difference in amplitude between the different peaks is smaller. However, a shape factor $\tau_{\operatorname{Im}}=-15$ was required to fit the frequency of the peaks in the predicted sound absorption coefficient to the measured sound absorption coefficient. The influence of the real part and imaginary part of the impedance on the sound absorption coefficient for a structure with a single tube resonator is discussed in Paper D, [18]. The influence of the shape factors $\tau_{\mathrm{Re}}$ and $\tau_{\mathrm{Im}}$ correspond to these conclusions. However, more research is needed to obtain a better understanding as to why these shape factors are required, since the impedance is based on measurements of the same material.

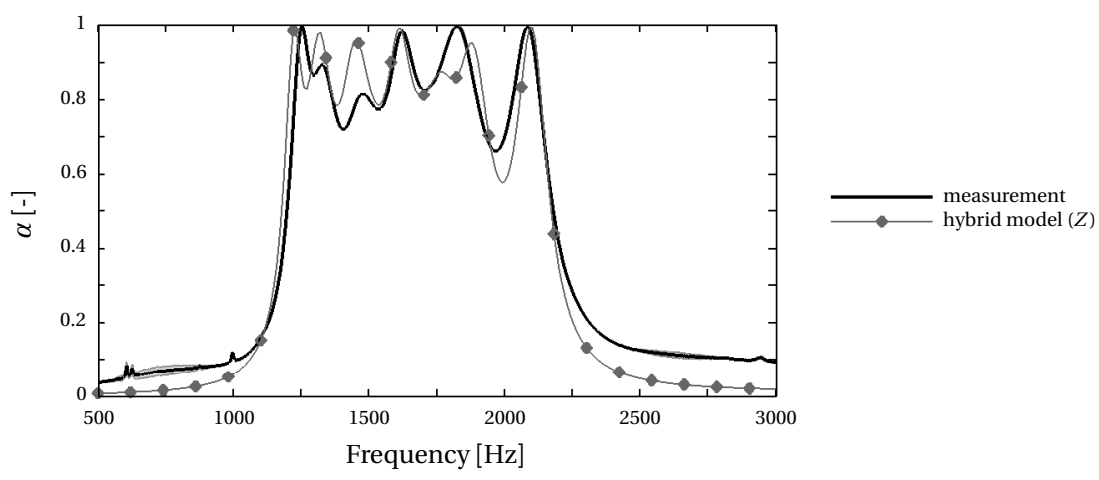

Figure 2.30: Measured sound absorption coefficient for a structure with coupled tube resonators and predicted sound absorption coefficient with a hybrid model including impedance boundary conditions.

Nonetheless, the results in Figure 2.30 show that this adjusted hybrid modelling 
approach can be used to predict the sound absorption coefficient of complex structures of tube resonators. Furthermore, the influence of the additional sound absorption of the material can be predicted by implementing an impedance to the boundaries of the structure in the numerical model. 


\section{3}

\section{Conclusions and recommendations}

As defined in Section 1.4, the main goal of this research was:

Developing a modelling approach to predict the sound absorption coefficient of porous structures and granular materials for normal and oblique incident sound waves.

The main goal has been divided into five objectives, which have been discussed together with the methods in Chapter 2 and the four (journal) articles in Part II of this dissertation.

\subsection{Conclusions}

A hybrid analytical/numerical modelling approach to predict the sound field in and above porous or granular structures has been developed. The modelling approach includes the viscothermal loss mechanisms inside the air-filled pores of this structure. Furthermore, the structure itself is modelled as a sound hard and rigid matrix to include the scattering of the incident waves. The developed modelling approach can be used to predict the sound absorption coefficient for normal and oblique incidence for three-dimensional and complex structures. An important advantage of this modelling approach compared to a fully numerical modelling approach is that the computational time is low, since:

- the viscothermal behaviour is included analytically, so no mesh refinement is needed in the finite element model to compute the particle velocity in the boundary layers correctly

- the scattering problem is concentrated locally around the porous or granular structure, so the air domain surrounding this structure can be modelled on a relatively small scale.

The developed modelling approach has been validated for normal incidence and oblique incidence. For normal incidence, the impedance tube technique has been used. The modelling approach has been validated for structures with tube resonators as well as for various structures of stacked glass marbles in two different 
sizes. The measured sound absorption coefficient was predicted extremely well for the tube resonators and the structures with the smaller sized marbles.

The validation for oblique incident sound waves was more complex, since measuring the sound absorption coefficient for oblique incidence introduces many uncertainties. However, the predicted and measured sound absorption coefficient for a box filled with glass marbles showed similar behaviour and trends.

One of the applications of this modelling approach is as a design tool for optimising the sound absorption coefficient of new porous road surfaces. In this dissertation a demonstration is made of how the developed modelling approach can be used to predict the influence of road parameters such as the porosity, layer thickness and stone size on the sound absorption coefficient for normal and oblique incidence. Two prototype road surfaces have been designed based partly on the design criteria resulting from these predictions and partly on existing knowledge from literature and measurements. The sound absorption coefficient of these two prototype tracks was measured both for normal incidence as well as for oblique incidence. For normal incidence, the sound absorption coefficient was large for all frequencies, especially in comparison with the standard reference road surface, a road surface with a low sound absorption coefficient. The measurements for oblique incidence show that the peak in amplitude of the sound absorption coefficient shifts to higher frequencies for an increasing angle of incidence, which was also predicted by the model simulations. Furthermore, the results show that the sound absorption coefficient of porous asphalt concrete indeed depends on both the frequency and the angle of incidence.

While the developed modelling approach is used only for simple packings of stacked spheres, the predicted trends for certain road parameters are confirmed by the measurements. Therefore, the approach can be used as a tool to optimise the sound absorption coefficient of porous road surfaces based on trends, but cannot predict the absolute value of the sound absorption coefficient for new road surfaces. Moreover, with some additional research, the developed approach can be combined with existing Tyre Road Noise (TRN) model, a numerical model to predict the sound radiation of a rolling tyre in a transient simulation. The sound absorption of the road surface is included in a limited manner and does not depend on frequency or angle of incidence. Combining the TRN model with the hybrid modelling approach is expected to improve the predictions of the sound radiation of a rolling tyre, since the sound absorption coefficient predicted with this modelling approach depends on both the frequency and the angle of incidence.

Another application of modelling approach is to predict and optimise the sound absorption coefficient for tube resonators or structures of coupled tube resonators. Two types of resonator structures have been designed and optimised for broadband sound absorption using analytical relations and the modelling approach. Both structures are measured in the impedance tube to determine the sound absorption coefficient. The first resonator structure was made from solid aluminium and the behaviour was predicted accurately by the hybrid model of the same structure. 
The second resonator structure was manufactured with a 3D printing technique: Selective Laser Sintering (SLS). The printed material was slightly porous and sound absorbing, which caused the resonance peaks in the sound absorption coefficient to shift to lower frequencies than the estimated sound absorption for this structure with sound hard boundaries. The modelling approach has been adjusted to include the impedance of the material. Using this adjusted modelling approach, the sound absorption coefficient was predicted very well. Therefore, the developed modelling approach can be used to optimise the design of complex structures manufactured with SLS, including the additional sound absorption of the material itself.

\subsection{Recommendations}

Currently, the hybrid modelling approach is used as a design tool for porous asphalt concrete based on a structure of stacked spheres, instead of a more realistic implementation of the stone matrix of the asphalt concrete. The numerical model of the structure will be more realistic when combinations of different sizes and shapes of the stones are included. This can be implemented using tools that generate three-dimensional structures of different sizes and shapes or based on scans of existing road surfaces, for example a computed tomography (CT) scan. Another recommendation is to extend the model with multiple air layers with different viscothermal properties such that multi-layered asphalt concrete can be modelled as well.

To accommodate road manufacturers, a database with model results combined with a graphical user interface should be developed, such that the model itself, and thus the required software, is no longer needed when designing new road surfaces.

Furthermore, when the existing TRN model is combined with the developed modelling approach, the predictions of the sound radiation of a rolling tyre will improve. In the TRN model, the sound radiation is modelled using the BEM method. It is expected that it is sufficient to model the road surface with a surface impedance, which depends both on the frequency and on the angle of incidence. This surface impedance can be found with the hybrid modelling approach as a separate step. 



\section{Bibliography}

[1] Regulation (EC) No 1222/2009 of the European Parliament and of the Council. Official Journal of the European Union, November 2009.

[2] ISO 10844:2014(en) Acoustics - Specification of test tracks for measuring noise emitted by road vehicles and their tyres, 2014 .

[3] ISO 11819-2:2017(en) Acoustic - Measurement of the Influence of road surfaces on traffic noise - Part 2: The close-proximity method, 2017.

[4] J. Allard and N. Atalla. Propagation of Sound in Porous Media: Modelling Sound Absorbing Materials 2e. John Wiley \& Sons, 2009.

[5] N. Atalla, F. Sgard, and C. K. Amedin. On the modeling of sound radiation from poroelastic materials. J. Acoust. Soc. Am., 120:1990-1995, 2006.

[6] K. Attenborough, I. Bashir, and S. Taherzadeh. Outdoor ground impedance models. J. Acoust. Soc. Am., 129:2806-2819, 2011.

[7] K. Attenborough, K. M. Li, and K. Horoshenkov. Predicting outdoor sound. Taylor \& Francis, 2007.

[8] Seismic Audio. Studio acoustic foam sheets.

[9] D. Bekke. Engineering tools for interior tyre tread pattern noise. $\mathrm{PhD}$ thesis, University of Twente, 62014.

[10] D. Bekke, Y. H. Wijnant, T. Weegerink, and A. de Boer. Tire-road noise: an experimental study of tire and road design parameters. In Noise Control for Quality of Life, pages 564-570. Austrian Noise Abatement Association, 92013.

[11] D. A. Bekke, Y. H. Wijnant, D. Schipper, B. Bobbink, G. Oude Lansink, and R. Stevens. Silent and Safe Roadtraffic-project: An optimization of the tyreroad interaction on noise and wet grip. In Euronoise 2015, pages 1345 - 1350, June 2015.

[12] W. M. Beltman. Viscothermal wave propagation including acousto-elastic interaction. PhD thesis, University of Twente, 1998.

[13] M. C. Berengier, M. R. Stinson, G. A. Daigle, and J. F. Hamet. Porous road pavements: Acoustical characterization and propagation effects. J. Acoust. Soc. Am., 101(1):155-162, 1997.

[14] M. Bezemer-Krijnen, Y. H. Wijnant, and A. de Boer. Experimental validation of a modelling approach to predict sound absorption for porous asphalt roads. In Proceedings of ISMA2016 including USD2016, 2016.

[15] M. Bezemer-Krijnen, Y. H. Wijnant, and A. de Boer. Predicting sound absorption of stacked spheres: combining an analytical and numerical approach. Acta Acustica united with Acustica, 102:971-983, August 2016.

[16] M. Bezemer-Krijnen, Y. H. Wijnant, and A. de Boer. Tyre-road noise measurements: influence of tyre tread and road characteristics. In Inter-noise 2016, 2016.

[17] M. Bezemer-Krijnen, Y. H. Wijnant, and A. de Boer. Integral approach to 
tire/road noise: considering both tire and road design. Submitted, 2018.

[18] M. Bezemer-Krijnen, Y. H. Wijnant, and A. de Boer. Modelling approach for 3d printed sound absorbing panels. Submitted, 2018.

[19] M. Bezemer-Krijnen, Y. H. Wijnant, and A. de Boer. Prediction of sound absorption of stacked granular materials for normal and oblique incident sound waves. Acta Acustica united with Acustica, 104(3):464-476, May/June 2018.

[20] K. P. Biligiri, B. Kalman, and A. Samuelsson. Understanding the fundamental material properties of low-noise poroelastic road surfaces. International Journal of Pavement Engineering, 14(1):12-23, 2013.

[21] J. Billingham and A. C. King. Wave Motion. Cambridge University Press, reprinted (2006) edition, 2000.

[22] M. A. Biot. Theory of propagation of elastic waves in a fluid-saturated porous solid. i. low-frequency range. Acoustical Society of America, 28(2):168-178, March 1956.

[23] M. A. Biot. Theory of propagation of elastic waves in a fluid-saturated porous solid. ii. higher frequency range. Acoustical Society of America, 28(2):179-191, March 1956.

[24] D. T. Blackstock. Fundamentals of physical acoustics. John Wiley \& Sons, 2000.

[25] M. Brinkmeier, U. Nackenhorst, S. Petersen, and O. VonEstorff. A finite element approach for the simulation of tire rolling noise. J. Sound Vib., 309(1):20-39, 2008.

[26] Y. Champoux and M. R. Stinson. On acoustical models for sound propagation in rigid frame porous materials and the influence of shape factors. J. Acoust. Soc. Am., 92:1120-1131, 1992.

[27] Y. de Kluizenaar, E. M. Salomons, S. A. Janssen, F. J. van Lenthe, H. Vos, H. Zhou, H. M. E. Miedema, and J. P. Mackenbach. Urban road traffic noise and annoyance: The effect of a quiet façade. The Journal of the Acoustical Society of America, 130(4):1936-1942, 2011.

[28] M. E. Delany and E. N. Bazley. Acoustical properties of fibrous absorbent materials. Appl. Acoust., 3(2):105-116, 1970.

[29] E. A. M. Franssen, J. E. F van Dongen, J. M. H. Ruysbroek, H. Vos, and R. Stellato. Hinder door milieufactoren en de beoordeling van de leefomgeving in Nederland, Inventarisatie Verstoringen 2003.

[30] M. H. C. Hannink. Acoustic resonators for the reduction of sound radiation and transmission. PhD thesis, University of Twente, 2007.

[31] M. H. C. Hannink., Y. H. Wijnant, and A. de Boer. Optimised sound absorbing trim panels for the reduction of aircraft cabin noise. In Eleventh International Congress on Sound and Vibration, ICVS 11, pages 1855-1862, 2004.

[32] K. Iwao and I. Yamazaki. A study on the mechanism of tire/road noise. JSAE review, 17(2):139-144, 1996. 
[33] D. L. Johnson, J. Koplik, and R. Dashen. Theory of dynamic permeability and tortuosity in fluid-saturated porous media. J. Fluid Mech., 176:379 - 402, 1987.

[34] P. Kindt. Structure-borne tyre/road noise due to road surface discontinuities. $\mathrm{PhD}$ thesis, Katholieke Universiteit Leuven, Leuven, Belgium, 2009.

[35] A. B. Knol and B. A. M. Staatsen. Trends in the environmental burden of disease in the Netherlands, 1980 - 2020, 2005.

[36] W. Kropp, P. Sabiniarz, H. Brick, and T. Beckenbauer. On the sound radiation of a rolling tyre. J. Sound Vib., 331(8):1789-1805, 2012.

[37] E. R. Kuipers. Measuring sound absorption using local field assumptions. $\mathrm{PhD}$ thesis, University of Twente, 2013.

[38] E.R. Kuipers, Y.H. Wijnant, and A. de Boer. Measuring sound absorption: considerations on the measurement of the active acoustic power. Acta Acustica united with Acustica, 100(2):193 - 204, 2014.

[39] U. J. Kurze. Noise reduction by barriers. The Journal of the Acoustical Society of America, 55(3):504-518, 1974.

[40] K. Larsson, S. Barrelet, and W. Kropp. The modelling of the dynamic behaviour of tyre tread blocks. Appl. Acoust., 63(6):659-677, 2002.

[41] B. Makwana, D. A. Bekke, B. de Bruijn, and SKP Amarnath. Modeling tools for the development of the safe and silent tyres. In EuroNoise 2015, pages 1025 1029, June 2015.

[42] F. P. Mechel. Sound fields at periodic absorbers. J. Sound Vib., 136(3):379-412, 1990.

[43] M. Mokhtari. The effect of a tribo-modified surface layer on friction in elastomer contacts. PhD thesis, University of Twente, 2015.

[44] M. Mokhtari, D. J. Schipper, and T. V. Tolpekina. On the friction of carbon black- and silica-reinforced br and s-sbr elastomers. Tribology Letters, 54(3):297-308, 42014.

[45] O. Mouraille, W. A. Mulder, and S. Luding. Sound wave acceleration in granular materials. Journal of Statistical Mechanics: Theory and Experiment, 2006(07):P07023, 2006.

[46] M. J. J. Nijhof. Viscothermal Wave Propagation. PhD thesis, University of Twente, 2010.

[47] World Health Organization et al. Burden of disease from environmental noise: Quantification of healthy life years lost in Europe. In Burden of disease from environmental noise: Quantification of healthy life years lost in Europe, pages 126-126. 2011.

[48] U. Sandberg. Road traffic noise - The influence of the road surface and its characterization. Appl. Acoust., 21(2):97-118, 1987.

[49] U. Sandberg and J. A. Ejsmont. Tyre/road noise reference book. 2002.

[50] U. Sandberg and L. Goubert. PERSUADE: a European project for exceptional noise reduction by means of poroelastic road surfaces. In 40th International 
Congress and Exposition on Noise Control Engineering 2011 (INTER-NOISE 2011), pages 673-684, 2011.

[51] J. H. Schutte. Numerical Simulation of Tyre/Road Noise. PhD thesis, University of Twente, 2011.

[52] M. R. Stinson. The propagation of plane sound waves in narrow and wide circular tubes, and generalization to uniform tubes of arbitrary cross-sectional shape. Acoustical Society of America, 89(2):550-558, February 1991.

[53] M. R. Stinson and Y. Champoux. Propagation of sound and the assignment of shape factors in model porous materials having simple pore geometries. $J$. Acoust. Soc. Am., 91(2):685-695, 1992.

[54] H. Tijdeman. On the propagation of sound waves in cylindrical tubes. $J$. Sound Vib., 39(1):1-33, 1975.

[55] Tubantia.nl. Twente airport kan door na verworpen bezwaren, 2017.

[56] F. J. M. van der Eerden. Noise reduction with coupled prismatic tubes. $\mathrm{PhD}$ thesis, University of Twente, 2000.

[57] C. Zwikker and C. W. Kosten. Sound absorbing materials. Elsevier, 1949. 


\section{Part II}

\section{Papers}





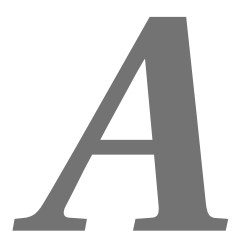

\section{Predicting sound absorption of stacked spheres: combining an analytical and numerical approach}

This chapter is based on the paper 'Predicting Sound Absorption of Stacked Spheres: Combining an Analytical and Numerical Approach', [15], published in: 'Acta Acustica united with Acustica', Vol. 102 (2016) 971 - 983. Copyright by (c) (2016) S. Hirzel Verlag/European Acoustics Association, http//dx.doi.org/10.3813/AAA.919012. Readers must contact the publisher for reprint or permission to use the material in any form. 


\title{
Predicting sound absorption of stacked spheres: combining an analytical and numerical approach
}

\author{
M. Bezemer-Krijnen ${ }^{1)}$, Y.H. Wijnant ${ }^{1)}$, A. de Boer ${ }^{1)}$ \\ Acta Acustica united with Acustica, Volume 102, Number 6, November/December 2016 \\ 1) Faculty of Engineering Technology, University of Twente, P.O. Box 217, 7500 AE Enschede, \\ The Netherlands. \\ m.bezemer@utwente.nl
}

\begin{abstract}
Tire/road noise is a serious problem, but can be significantly reduced by the use of porous asphalt concrete. Here, the sound absorption of the porous asphalt concrete is important and can be predicted by ground impedance models. Yet, modeling porous asphalt concrete is complex, especially when nonlocal effects and scattering effects are considered. The objective of this research is to predict the sound absorption coefficient for a three-dimensional porous structure. The proposed solution is obtained using a novel modeling approach, in which the total solution of the sound field is found by combining the solutions of two subsystems: a background sound field and a scattered sound field. The background sound field contains the (analytical) solution of the sound field including the viscothermal energy dissipation inside the pores of the porous asphalt concrete. In the second subsystem, the (numerical) solution for the scattering on the rigid stone skeleton of the pavement is found. For both subsystems, we use a model containing two layers: an air layer and a viscous air layer with a certain granular structure. The main advantage of this modeling approach is the (relatively) low computation time. In this paper, the proposed modeling approach and the validation of this approach are described. The modeling approach is validated for normal incident plane waves absorbed and scattered by various structures of stacked marbles, using the impedance tube technique. This approach can be applied to predict the absorption coefficient of porous structures, like asphalt concrete roads. Moreover, it can be used as design tool to optimize the sound absorption of new road surfaces.
\end{abstract}




\section{Introduction}

Tire/road noise is a serious problem in (densely) populated areas. Much research has been done to predict both the sound radiation from rolling tires and the sound absorption of ground surfaces.

Sound propagation above ground surfaces and sound absorption of ground surfaces can be implemented in a BEM model, by modeling an impedance plane using the half-space notation (e.g. $[18,20])$. However, this assumes a locally reacting surface while porous asphalt concrete is best described as an extended reacting surface with hard backing [5, pp. 39-50]. This can be implemented using a multi-domain BEM approach, in which the air and the porous asphalt concrete are modelled as two coupled domains (e.g. $[2,3,19])$.

For both methods - the impedance plane method and the two-domain BEM approach - the acoustic properties of the porous asphalt concrete have to be described. This is generally done using a ground impedance model, in which a distinction is made between (semi-) empirical, phenomenological models and microstructural models. In (semi-) empirical models, the model parameters are fitted to measurement results. For the majority of these models, only one or two parameters have to be known or measured. A well-known empirical ground impedance model is the Delany and Bazley model [11]. In this model, the impedance and sound absorption coefficient are described as function of the frequency and flow-resistance. This model is not directly applicable to porous asphalt concrete, since Delany and Bazley mainly tested fibrous materials with a porosity close to one. However, with some adjustments, the model gives acceptable results for outdoor surface impedances $[5, \mathrm{pp}$. 62-63].

In phenomenological models, the phenomena inside the porous material are considered on a global scale and the parameters needed for these models are often determined by measurements. It is possible to include viscothermal effects in phenomenological models. The
Hamet model (e.g. [5, 8, 12]) is an extended phenomenological model, for which only three parameters are needed to describe the material. The Hamet model is often used to describe porous asphalt concrete and includes frequency dependent viscous and thermal effects.

The last type of ground impedance models are the microstructural models. This model approach is based on analytical descriptions of the (simplified) microstructure of the porous material, such as pores filled with air in an elastic or rigid frame. The pressure and velocity inside these pores are described analytically and both the thermal and the viscous dissipation of energy are included. Many microstructural models are available. More information about microstructural models is given by e.g. $[1,5]$.

Since there are many ground impedance models available and it is not within the scope of this paper to describe them all, the reader is referred to the overview given in [4]. In this overview, it is stated that one of the difficulties with ground impedance models are the number of parameters needed to describe the porous material accurately. When the authors [4] choose the most suitable ground impedance model, this choice was partly based on the limited number of parameters needed to describe porous asphalt surfaces.

A method to find these parameters without measurements, is to combine a ground impedance model with an additional model describing a repetitive structure that represents the porous material. This extra model can be based on analytical relations, as described by [22] for a packing of spheres, or based on a finite element model $[16,25]$. The advantage of using finite element methods is that more complex geometries of the repetitive structure can be used.

\subsection{Objective}

The objective of this research is to develop a modeling approach which can be used to predict the acoustic behavior, such as sound ab- 
sorption, for porous roads in the design phase. In this approach, the porous asphalt concrete should be modelled including local effects such as viscothermal energy dissipation inside the pores between the stones and scattering on the stones within the road surface.

The common denominator of most existing models and modeling approaches is that they consider the porous road surface as a homogeneous medium, for which the acoustic properties are valid for the entire porous road surface. In these models, the pressure and particle velocity are defined for a single pore within a rigid or flexible bulk material. The properties obtained for an individual pore are then used to find a generalized model for the entire porous material, for example by using shape factors and the porosity of the material. Therefore, the properties of the individual pore segments and pores are averaged; the porous medium is assumed to be homogeneous.

With these models, the effects of local behavior cannot be distinguished and the behavior for oblique incident waves is fixed. However, rolling tires will radiate sound in all directions and the effects of the nonlocal surface behavior should be included.

One possible modeling approach to predict the effects of nonlocal effects and behavior for oblique incidence is given by [17] for an absorber with a periodic structure, which can be a rigid frame with slits filled with absorbing material. The author of this paper describes, analytically, the scattered waves and reflected waves in terms of Hartree harmonics. While this modeling approach takes into account the nonlocal behavior of the material, it is quite complex to apply it for porous asphalt concrete, since the geometry has to be described by Hartree harmonics.

Therefore, the method we propose is to solve the scattered sound field using a finite element model. This modeling approach combines the analytical description of an undisturbed background sound field, including a viscous medium, with a finite element model to include the scattering on the stones in the porous asphalt concrete. In this approach, the proper- ties of the viscous layer are based on the properties of the sound field inside a single pore. The approach and the resulting three-dimensional model are described in this paper. With this model, it is possible to predict the sound absorption for specified structures within the asphalt concrete and for oblique incident waves. Furthermore, it can be used as design tool to study the influence of the stone sizes in asphalt gradings, the effect of multiple layers of different gradings and the absorption for oblique incident waves.

Note that the porous asphalt concrete is assumed to be rigid and vibrations introduced by the rolling of the tyre are not included. Also, possible coupling between acoustic vibrations and structural vibrations are not taken into account.

\subsection{Outline}

An overview of the developed modeling approach, implementation and an example is given in Section 2. The modeling approach is validated for normal incident plane waves using an impedance tube. Therefore, in this paper, we explain the modeling approach for normal incidence, but the developed approach can also be used for oblique incident waves, as is shown by [9]. Furthermore, we focus on the sound absorption of a simplified rigid granular structure, since the model is validated for various structures of stacked marbles, as described in Section 3 . The conclusions and recommendations are given in Section 4.

\section{Theory}

In Section 2.1, the modeling approach and the solution for the background sound field, the scattered sound field and the combined total sound field are discussed. The implementation of this approach is described in Section 2.2 and an example is given in Section 2.3. 


\subsection{Modeling approach}

A schematic view of the proposed modeling approach with the two subsystems is given in Figure 1.

The background sound field is solved analytically using a microstructural model and the scattered sound field is solved numerically using finite element methods. The background and scattered fields are summed to find the total solution, hence including both the viscothermal effects and the scattering effects.

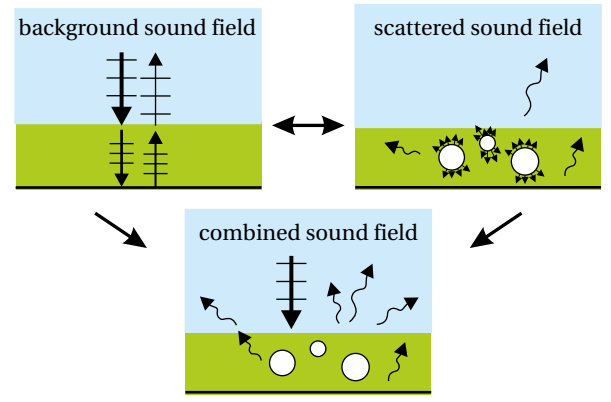

Figure 1: Schematic view of modeling approach

For both the background and scattered field, a model consisting of two layers with acoustically hard backing is implemented. The first layer consists of air and the second of viscous air. In the numerical model used for the solution of the scattered field, a schematic model of the stone structure is included in the viscous air layer. This stone structure represents the porous asphalt concrete and is modelled such that only the viscous air between the stones in this structure is considered.

Both the background and scattered sound field are solved by the Helmholtz equation [10]:

$$
\nabla^{2} \Phi+k_{i}^{2} \Phi=0
$$

where $\nabla$ is the Laplace operator, $\Phi$ is the velocity potential and $k_{i}$ is the complex wave number of the $i$ th layer. The wavenumber in the first layer, medium I, is $k_{\mathrm{I}}=k$; the wave number assuming standard air conditions. The wave number used in the Helmholtz equation of the second layer $k_{\mathrm{II}}$ (Equation 33) includes the viscothermal properties of this medium. These properties are based on the so-called low-reduced frequency (LRF) model (e.g. [6, 14, 21, 23]), which are described in more detail in Appendix A. The LRF approach is similar to that of a microstructural model approach, but can be applied to a broader scope of problems. Furthermore, the applications of the LRF approach are not limited to predicting the impedance and sound absorption of porous ground surfaces. Note that any ground impedance model, as long as the viscothermal energy dissipation is included, can be used to describe the properties of the viscous air layer in this proposed hybrid modelling approach.

However, in contrast to the usual implementation, in the proposed approach the characteristic impedance and viscothermal wave propagation coefficient for a single cylindrical pore are directly used in both subsystems. For the analytical solution of the background field this is also an homogenisation of the properties, but when combined with the scattered field the nonlocal effects of the porous asphalt concrete structure are included. Therefore, this modeling approach can be used to study the influence of various parameters during the design phase of porous asphalt road surfaces. One can think of the influence of stone sizes in asphalt gradings, the effect of multiple layers of different gradings and the influence of oblique incident waves on the sound absorption.

Another advantage of this method is that the computational time is kept relatively low. The background field is solved efficiently using analytical expressions and the evaluated volume in the finite element model is small, since the scattered sound field is local.

\subsubsection{Background sound field}

The background sound field is based on the description of the sound field in two mediums: medium I is assumed to have standard air properties and medium II is defined as a viscous air layer that includes viscothermal effects, but without the structure representing the porous asphalt concrete. The second medium 
has an acoustically hard backing. A schematic overview of the background field is shown in Figure 2.

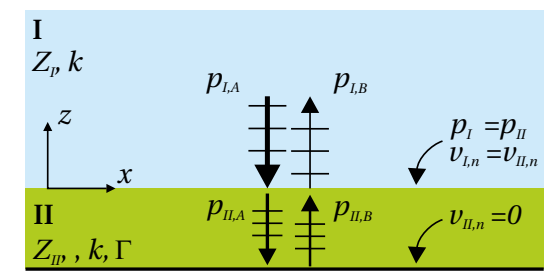

Figure 2: Schematic overview of the analytical model

The sound field in both mediums is described by the sound pressure and the particle velocity, using the positive time dependence $e^{i \omega t}$.

The sound pressure field in the air layer (medium I) for an incoming sound wave including transmission from the viscous air layer and the reflection at the interface between the two layers is described by $p_{\mathrm{I}}$ :

$$
p_{\mathrm{I}}=A_{\mathrm{I}} e^{i k z}+B_{\mathrm{I}} e^{-i k z}
$$

where $k$ is the wave number in medium I. The incident wave with complex amplitude $A$ is travelling in the negative $\mathrm{z}$-direction and the reflected waves with complex amplitude $B$ are travelling in the positive $\mathrm{z}$-direction. The particle velocity in the direction normal to the interface between medium I and medium II is given by $v_{\mathrm{I}, n}$ :

$$
v_{\mathrm{I}, n}=\mathbf{v}_{\mathrm{I}} \cdot \mathbf{n}=\frac{-1}{Z_{\mathrm{I}}}\left[A_{\mathrm{I}} e^{i k z}-B_{\mathrm{I}} e^{-i k z}\right]
$$

where $Z_{\mathrm{I}}$ is the characteristic impedance, $\mathbf{v}_{\mathrm{I}}$ is the particle velocity, both in medium I, and $\mathbf{n}$ is the unit vector normal to the interface between medium I and II. Medium I is assumed to have standard air properties, which yields $Z_{\mathrm{I}}=\rho_{0} c_{0}$, where $\rho_{0}$ is the density of air and $c_{0}$ the speed of sound in air.

The sound pressure field in the viscous air layer (medium II) is described by $p_{\mathrm{II}}$ :

$$
p_{\mathrm{II}}=A_{\mathrm{II}} e^{\Gamma k z}+B_{\mathrm{II}} e^{-\Gamma k z}
$$

where $\Gamma=i k_{\mathrm{II}} / k$ is the viscothermal wave propagation coefficient.
The particle velocity in z-direction for the viscous air layer is given by $v_{\mathrm{II}, n}$ :

$$
v_{\mathrm{II}, n}=\mathbf{v}_{\mathrm{II}} \cdot \mathbf{n}=\frac{G}{\rho_{0} c_{0}}\left[A_{\mathrm{II}} e^{\Gamma k z}-B_{\mathrm{II}} e^{-\Gamma k z}\right]
$$

where $\mathbf{v}_{\text {II }}$ is the particle velocity in medium II, and $\mathbf{n}$ is again the unit vector normal to the interface between medium I and II. The coefficient $G$, defined in Equation 31, depends on the shape of the pores and includes the viscothermal effects.

Provided that the properties of the acoustic propagation (i.e. $\Gamma$ and $G$ ) in the viscous air layer are known, the background sound field is completely defined once the complex amplitudes $A_{\mathrm{I}}, B_{\mathrm{I}}, A_{\mathrm{II}}$ and $B_{\mathrm{II}}$ are found.

The boundary conditions used to solve this problem are shown in Figure 2. The first two boundary conditions are the continuity of pressure and particle velocity in $\mathrm{z}$-direction, at the interface between medium I and medium II $(z=0)$. The third boundary condition is the acoustically hard backing at the bottom of the viscous air layer, at $z=-d$, where $d$ is the thickness of this layer. The particle velocity normal to this surface is equal to 0 . To summarize:

$$
\begin{aligned}
\left.p_{\mathrm{I}}\right|_{z=0} & =\left.p_{\mathrm{II}}\right|_{z=0} \\
\left.v_{\mathrm{I}, n}\right|_{z=0} & =\left.v_{\mathrm{II}, n}\right|_{z=0} \\
\left.v_{\mathrm{II}, n}\right|_{z=-d} & =0
\end{aligned}
$$

The solution of the background sound field then yields:

$$
\begin{aligned}
& B_{\mathrm{I}}=A_{\mathrm{I}} \frac{\left(1+e^{-2 \Gamma k d}\right)-G\left(1-e^{-2 \Gamma k d}\right)}{\left(1+e^{-2 \Gamma k d}\right)+G\left(1-e^{-2 \Gamma k d}\right)} \\
& A_{\mathrm{II}}=2 A_{\mathrm{I}} \frac{1}{\left(1+e^{-2 \Gamma k d}\right)+G\left(1-e^{-2 \Gamma k d}\right)} \\
& B_{\mathrm{II}}=A_{\mathrm{II}} e^{-2 \Gamma k d}
\end{aligned}
$$

where the complex amplitude of the incident wave in medium I, $A_{\mathrm{I}}$, is assumed known, since this amplitude is related to the wave excitation.

\subsubsection{Scattered sound field}

The second step in this modeling approach is solving the scattered sound field. We used a finite element method to find the sound pressure 
field due to the reflections on the structure of spheres. This step is indicated on the top right side of Figure 1.

The stone skeleton of the porous asphalt concrete is simplified to a structure of stacked spheres. The spheres are considered to be sound hard objects. Based on the analytical expressions of the background velocity (Equations 3 and 5) we can calculate the particle velocity due to scattering $v_{\text {scat }}$ at the surface of the spheres:

$$
v_{\text {scat }}=-\mathbf{v} \cdot \mathbf{n}
$$

where $\mathbf{v}=\mathbf{v}_{\text {I }}$ for $z \geq 0, \mathbf{v}=\mathbf{v}_{\text {II }}$ for $z<0$, $\mathbf{n}$ is the unit vector normal to the surface of the spheres; the tangent component is neglected. For medium II the background velocity $\mathbf{v}_{\text {II }}-$ including the viscothermal effects - can be written as:

$$
\mathbf{v}_{\mathrm{II}}=\frac{G}{\rho_{0} c_{0}}\left[\mathbf{R} A_{\mathrm{II}} e^{\Gamma k \xi}-\mathbf{R} B_{\mathrm{II}} e^{-\Gamma k \xi}\right]
$$

Note that the background particle velocity is a vector, the components depend on the rotation vector:

$$
\mathbf{R}=\{\sin \theta \cos \varphi, \sin \theta \sin \varphi, \cos \theta\}^{\prime}
$$

The spatial coordinate $\xi$ is given by:

$$
\xi=x \sin \theta \cos \varphi+y \sin \theta \sin \varphi+z \cos \theta
$$

where $x, y, z$ are the coordinates of a point on the outer surface of the spheres. The coordinates, the polar angle $\theta$ and azimuthal angle $\varphi$ are according to the conventions shown in Figure 3.

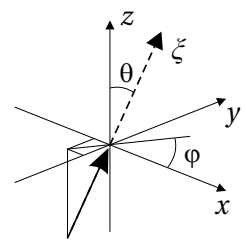

Figure 3: Conventions of direction, polar angle $\theta$ and azimuthal angle $\varphi$.

The solution of the total sound field is found when the background field and the scattered field are summed.

\subsection{Implementation}

In Section 3, the model approach is validated for stacked marbles in an impedance tube. Therefore, the modeling approach is implemented for a structure of stacked spheres.

\subsubsection{Schematic modeling of stone struc- tures}

The structure with the stacked spheres is a simplified representation of the stone (and bitumen) skeleton of porous asphalt concrete. At this moment, the spheres in the model all have the same dimensions and the structure of the stacked spheres is based on the hexagonal close-packed (hcp) sphere packing. In a hcp packing, the spheres are stacked in layers that have the same packing in every layer and can be written as A-B-A-B-A-B-... Each sphere is surrounded by 12 other spheres. The hcp sphere packing is shown in Figure 4.
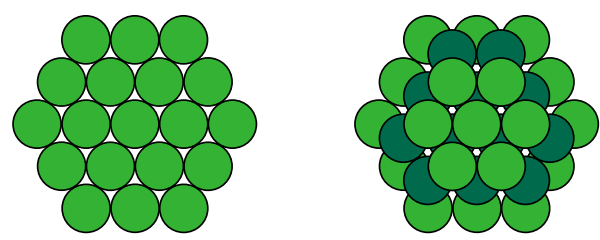

Figure 4: Stacked spheres in single layer (left) and multiple layers in hcp packing (right).

In the future, we will implement structures which will resemble that of porous asphalt concrete more. Also, the model will be extended with additional layers, such that the sound absorption for multilayered asphalt concrete can be evaluated.

\subsubsection{Properties of viscous layer}

The properties of the viscous air layer (medium II), and thus the air in between the spheres, are determined using a microstructural model. We used the low reduced frequency (LRF) model, but any ground impedance model that includes the viscothermal energy dissipation will work. The LRF model is described in more detail by [6, 
13, 21, 23]; some important relations are summarized in Appendix A. The viscothermal wave propagation coefficient $\Gamma$, coefficient $G$, characteristic impedance $Z_{\mathrm{II}}$ and speed of sound $c_{\mathrm{II}}$ (Equations 30, 31, 35 and 36, respectively) are derived for a prismatic microstructure with circular cross-section.

The characteristic radius of this cylindrical tube is based upon the so called hydraulic tube radius $r_{t}$. We derived the hydraulic radius based on the ratio between the volume of air between the spheres and the surface area of the spheres, the 'wetted surface'. The hydraulic radius is derived for a unit cell with the hcp structure, which is defined as the smallest possible repeatable unit, containing all structural and symmetry information. For the hcp structure, the total number of spheres in the unit cell is 6 . The unit cell is shown in Figure 5.
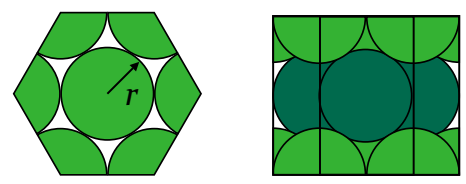

Figure 5: Schematic top view (left) and side view (right) of a unit cell for hcp packing

The hydraulic radius then yields:

$$
\begin{aligned}
r_{t} & =\frac{2 V_{\text {open }}}{A_{\text {wetted }}}=\frac{2(24 \sqrt{2}-8 \pi) r^{3}}{24 \cdot \pi r^{2}} \\
& =\left(\frac{2 \sqrt{2}}{\pi}-\frac{2}{3}\right) r
\end{aligned}
$$

where $V_{\text {open }}$ is the volume between the spheres, $A_{\text {wetted }}$ wetted surface and $r$ is the radius of the spheres in the unit cell.

The length of the characteristic tube and the porosity of the structure are not included as parameters in the viscous layer, since these follow directly from the FEM model of the stone structure. Therefore, the only variable needed to determine the properties of the viscous layer is the hydraulic radius of the characteristic tube.

\subsubsection{Absorption coefficient}

The proposed modeling approach can be used to predict the sound absorption behavior of a porous structure. The area averaged absorption coefficient $\alpha$ over an interface $S$, parallel to the interface between the medium I and medium II, is defined as the ratio of the time-averaged incident sound power $W_{\text {in }}$ which is absorbed by this area, as described by [24]:

$$
\alpha=\frac{W_{\mathrm{ac}}}{W_{\mathrm{in}}}=\frac{\int \mathbf{I}_{\mathrm{ac}} \cdot \mathbf{n} d S}{\int I_{\mathrm{in}} d S}
$$

where $W_{\mathrm{ac}}$ is the time-averaged active sound power at $S$ and $\mathbf{n}$ is the unit vector normal to the interface $S$. The active sound power is obtained by integration of the active intensity $\mathbf{I}_{\mathrm{ac}}$ over the interface $S$, and the incident sound power by integration of the incident intensity $I_{\text {in }}$ over $S$.

The active intensity can be obtained directly from the model:

$$
\mathbf{I}_{\mathrm{ac}}=\frac{1}{2} \operatorname{Re}(p \overline{\mathbf{v}})
$$

where $\overline{\mathbf{v}}$ denotes the complex conjugate of the complex particle velocity.

The complex sound pressure $p$ is the combination of the background pressure $p_{\text {bw }}$, according to Equations 2 and 4 for the analytically derived background field, and the scattered pressure $p_{\text {scat }}$, found with the numerical model:

$$
p=p_{\mathrm{bw}}+p_{\text {scat }}
$$

The incident intensity cannot be determined directly, but can be described in terms of the complex amplitude $A$.

$$
I_{\text {in }}=\frac{A \bar{A}}{2 \rho_{0} c_{0}}
$$

where $\bar{A}$ denotes the complex conjugate of $A$. The complex amplitude for the incident wave $A$ is defined by:

$$
A=\frac{1}{2}\left(p-\rho_{0} c_{0} v_{n}\right)
$$

where $p$ is the sound pressure and $v_{n}$ the particle velocity in normal direction. Note that it is assumed that the sound field can be described by local plane waves. 


\subsection{Example}

Using the described modeling approach, a three-dimensional numerical model of an impedance tube is developed. The numerical model contains:

- Medium I (air layer): modelled as a cylinder, with perfectly matched layers (PML) at the top side.

- Medium II (viscous air layer): modelled as the air between stacked spheres.

- The walls and bottom of the cylinder representing the impedance tube are assumed acoustically hard.

The scattering problem is solved using finite elements. The model is built using the multiphysics package Comsol (version 4.4), in combination with Matlab (version 2013a). The problem is solved in the frequency domain using the MUltifrontal Massively Parallel sparse direct Solver (MUMPS); a stationary linear solver.

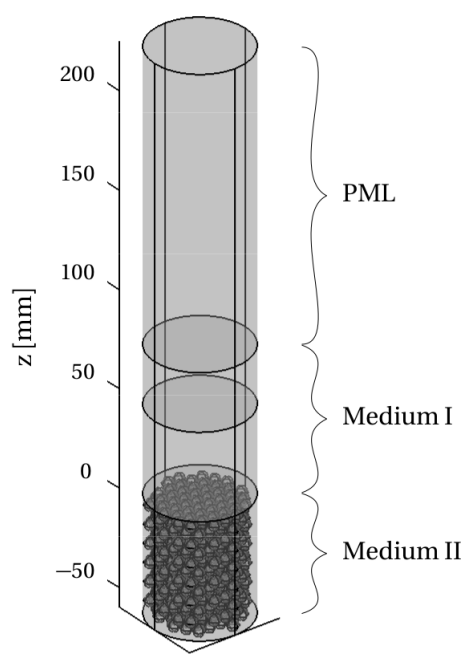

Figure 6: Geometry of numerical model with 12 layers of stacked $6 \mathrm{~mm}$ spheres.

A model is made according to the parameters listed in Table 2. The geometry of the model is shown in Figure 6 . The top of the impedance tube is enclosed by perfectly matched layers (PML), such that pressure waves entering these layers are completely absorbed without reflections. PML are introduced by [7] for the application of electromagnetic waves. Other boundary conditions at the top surface of medium I are also possible, for example an impedance boundary condition, where the impedance equals $\rho_{0} c_{0}$.

The height of medium I has to be sufficient large, such that local scattering of the sound waves, due to discretisation errors in the numerical model, are damped out at the location where the absorption coefficient is calculated.

Medium I represents the air layer and medium II represents the viscous air between the spheres. Note that this layer only contains the air between the stacked spheres, since the structure of spheres (as shown in Figure 7) is subtracted from the geometry of the impedance tube.

Since a hcp packing is used in the model, every other layer in z-direction is identical. The number of stones is given for the odd and even layers. The first layer is the top layer and the layers are numbered in negative z-direction. In Figure 7, the odd layers are shown in light green and the even layers in dark blue. The corresponding tube radius for this model is $r_{\mathrm{t}}=0.7 \mathrm{~mm}$. Using this tube radius, the viscothermal properties are derived according to Equations 30, 31, 35 and 36. Note that the LRF model returns frequency dependent properties.

\subsubsection{Mesh properties}

Furthermore, the mesh size of the model is studied and the meshing parameters are chosen such that for the generated mesh the solution is converged. Figure 8 shows the absorption coefficient for three variations in meshing parameters for the mesh of medium II, as listed in Table 1. From this figure can be concluded that the influence of the chosen meshing parameters on the absorption coefficient is neglectably small. 


\begin{tabular}{llll}
\hline & Model 1 & Model 2 & Model 3 \\
\hline Number of elements $\left(N_{e}\right)$ & 408746 & 496762 & 602554 \\
Tetrahedral elements & 408746 & 496762 & 602554 \\
Average element quality & 0.579 & 0.601 & 0.621 \\
Minimum element quality & $1.20 \mathrm{e}-4$ & $9.34 \mathrm{e}-5$ & $3.14 \mathrm{e}-4$ \\
Average grow rate & 2.051 & 1.965 & 1.904 \\
\hline
\end{tabular}

Table 1: Meshing parameters for medium II shown in Figure 8, all for model with 12 layers of stacked spheres with $D=6 \mathrm{~mm}$.

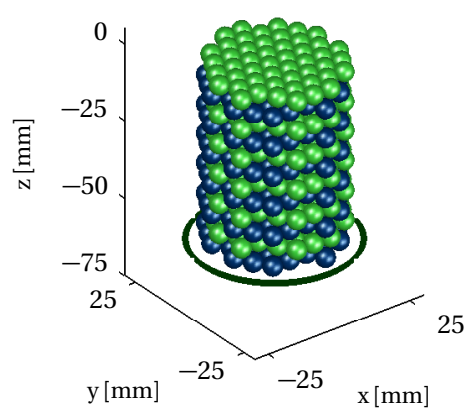

Figure 7: Stacked spheres of $6 \mathrm{~mm}$ diameter in hcp packing.

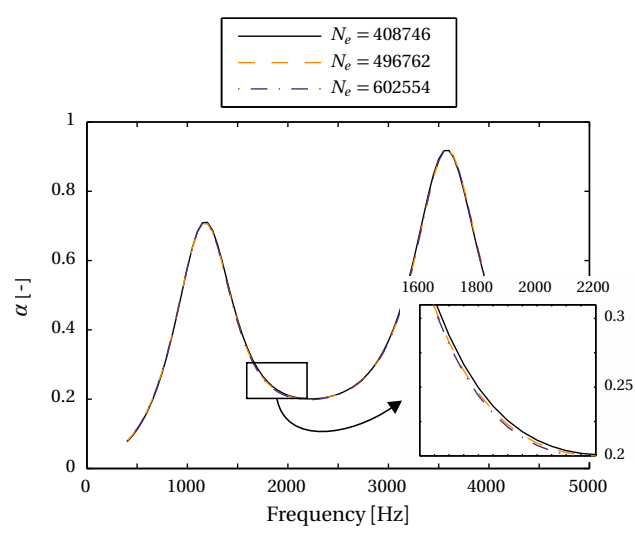

Figure 8: Absorption coefficient for three variations in meshing properties, all models contain 12 layers of stacked spheres.

It can also be concluded from Figure 8 that the mesh can be even coarser. However, meshing problems occurred while meshing the sphere structure. It is assumed that these are caused by the discretization of the spheres, as is illustrated in Figure 9. To avoid these problems, a finer mesh size is needed and a small overlap $\delta$ between the spheres is introduced, this is discussed in more detail in Section 3.1.2.

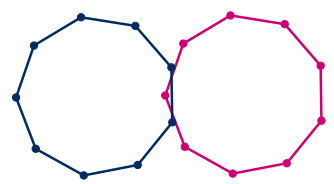

Figure 9: Discretization of the spheres.

\subsubsection{Results example model}

Figure 10 shows the solved sound pressure fields for incident plane waves in negative $\mathrm{z}$-direction, normal to the interface between medium I and II. The amplitude of the sound pressure field is shown for $f=3550 \mathrm{~Hz}$.

On the left in Figure 10, the combined incident and reflected sound pressure field for the background field, the analytical solution, is shown. The sound pressure field of the scattered field, found with the finite element model, is shown in the center of Figure 10.

The summation of both solutions yields the total sound field, as shown on the right in Figure 10. Figure 11 shows the absolute value of the total pressure along the center of the tube in $\mathrm{z}$-direction for several frequencies. The figure shows that the maximum pressure levels are found inside the viscous air layer $(z<0)$. Interesting is that for $f=2500 \mathrm{~Hz}$, where the sound absorption coefficient is low, the maximum and 
minimum values in the viscous air layer and the air layer are of the same amplitude.

Figures 12 and 13 show the real and imaginary part of the scattered and total sound field, respectively, in more detail. It can be seen that the pressure field is changed locally around the sphere structure. Again, the maximum and minimum pressure observed in the model results are found around the sphere structure in medium II.

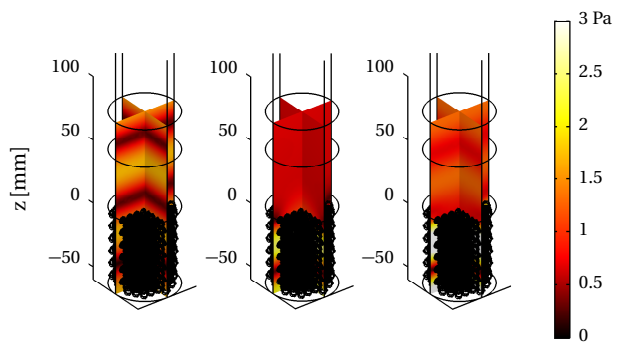

Figure 10: Solved sound pressure (amplitude) [Pa] for $D=6 \mathrm{~mm}, f=3550 \mathrm{~Hz}$ : (left) incoming and reflected sound pressure; (center) scattered sound pressure and (right) total sound pressure level.

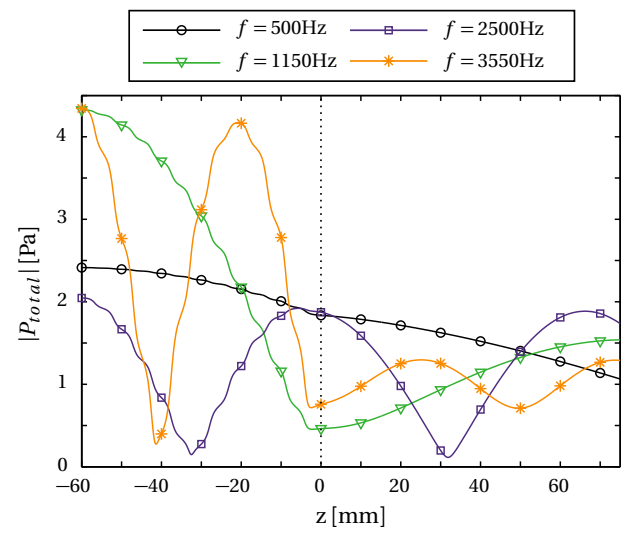

Figure 11: Total sound pressure (amplitude) for different frequencies.
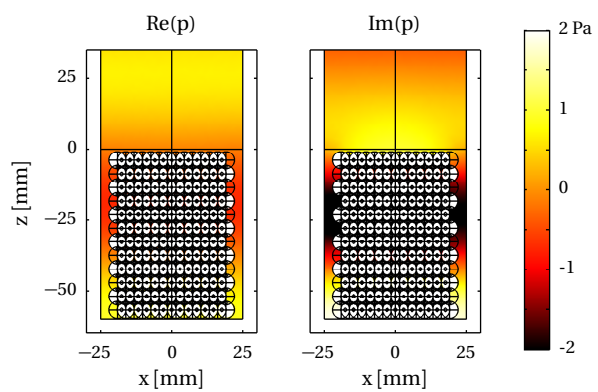

Figure 12: Detail of scattered sound pressure [Pa] for $D=6 \mathrm{~mm}, f=3550 \mathrm{~Hz}$.
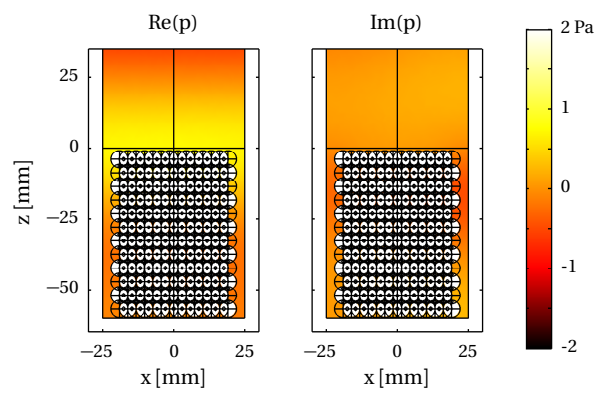

Figure 13: Detail of total sound pressure $[\mathrm{Pa}]$ for $D=$ $6 \mathrm{~mm}, f=3550 \mathrm{~Hz}$.

\begin{tabular}{lc}
\hline Parameter & Model A \\
\hline Impedance, medium I [kg/( $\left.\left.\mathrm{m}^{2} \mathrm{~s}\right)\right]$ & 413 \\
Speed of sound, medium I [m/s] & 343 \\
Diameter impedance tube [mm] & 50 \\
Layer height II [mm] & 60 \\
Diameter of spheres [mm] & 6 \\
Number of spheres, odd layers [-] & 51 \\
Number of spheres, even layers [-] & 50 \\
Effective tube radius $r_{\mathrm{t}}[\mathrm{mm}]$ & 0.7 \\
Spacing $\delta$ [mm] & -0.06 \\
\hline
\end{tabular}

Table 2: Parameters of model A (with spheres of $D=$ $6 \mathrm{~mm})$

\section{Validation with impedance tube}

The modeling approach is validated with absorption measurements for various structures 
of stacked marbles. Figure 14 shows the impedance tube used for these measurements. The aluminium impedance tube is closed with a sound hard aluminium plate of $10 \mathrm{~mm}$ thickness and placed (and stabilized) in upright position.

Glass marbles were placed inside the impedance tube, the tube was slightly shaken and the stack of spheres was pressed into a more compact packing using a heavy piston. The observed stacking was slightly different than the hcp packing, due to the circular shape and small diameter of the tube. Therefore, the number of marbles and the layer height are not exactly the same as the number of spheres and layer height of the sphere structure in the models. The number of marbles is estimated based on the weight of the marbles.

The measurement results for 4 different measurements are averaged to determine the sound absorption coefficient of the stacked marbles. Two series of two measurements were performed, for both series of two measurements, the stacking of spheres is removed and rebuilt and the microphones are demounted and mounted again. Per series, the microphones are switched from position. The measurement results show very good repeatability for all 4 measurements; an average standard deviation of 0.01 was found. Figure 15 shows the measurement results for the configuration with 12 layers of stacked marbles, where the shaded area indicates \pm 1 of the standard deviation. This figure also shows the results of the hybrid modeling approach ('Sim.', dashed line) and the results for a microstructural model by Champoux and Stinson [8], these results are discussed in Section 3.1.1.

The impedance tube has a build-in speaker and a microphone module containing upto $4 \mathrm{mi}$ crophones. More details about the setup and methods are given in Appendix B.

Since the maximum frequency is kept below the cutoff frequency of the tube, only plane wave propagation is possible. Therefore, using an impedance tube, the developed model can be validated only for normal incident waves. The validation for a structure of 12 layers of glass

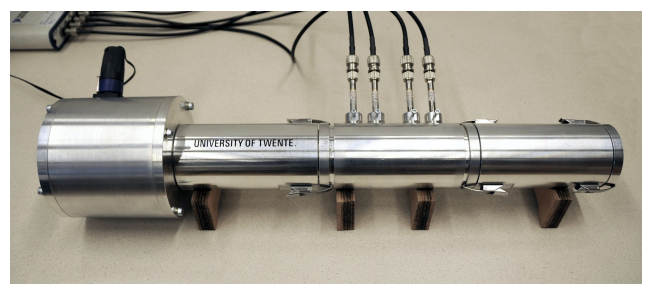

Figure 14: Impedance tube used for absorption measurements

marbles with a diameter of $6 \mathrm{~mm}$ is discussed in detail in Section 3.1. Here, also the sensitivity of the model results to the porosity and the tube radius used to estimate the viscothermal properties are discussed. The validation with a varying number of layers for marbles with a diameter of $6 \mathrm{~mm}$ and $12 \mathrm{~mm}$ is given in Section 3.2.

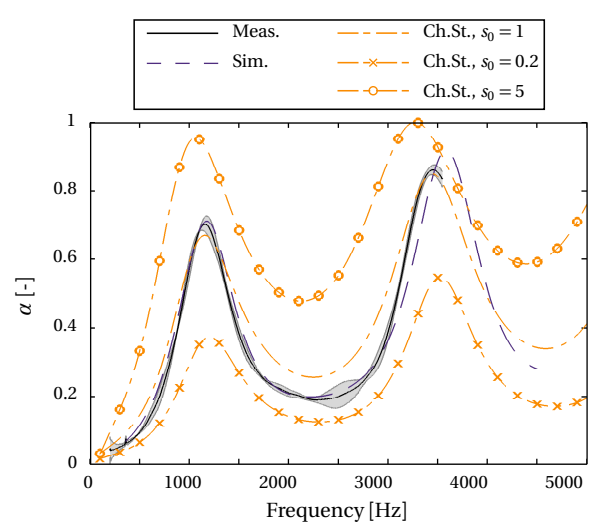

Figure 15: Measured absorption coefficient (shaded area indicates \pm 1 standard deviation) and predicted absorption coefficients, all for 12 layers of stacked marbles or spheres.

\subsection{Structure with 12 layers of $6 \mathrm{~mm}$ marbles}

The measured sound absorption coefficient for 12 layers of glass marbles of $6 \mathrm{~mm}$ diameter are compared to the example model from Section 2.3, which has a similar configuration. 
The measurement results for this sphere packing are shown in Figure 15. The results show two distinct peaks in the absorption coefficient, similar to the type of peaks as seen for tube resonators. The frequency of the first absorption peak is measured at $f \approx 1155 \mathrm{~Hz}$, which corresponds with a cylindrical tube resonator with a length of:

$$
L_{\text {eff }}=\frac{c_{0}}{4 f_{1}} \approx \frac{343}{4620}=74 \mathrm{~mm}
$$

where $L_{\text {eff }}$ is the effective length of the resonator. As expected, this length is larger than the total height of the structure with marbles, $H \approx 61 \mathrm{~mm}$, since the pores between the marbles are curved and the traveled path of the sound waves is longer than the height of the structure.

\subsubsection{Model results}

The measured and predicted sound absorption coefficient are shown in Figure 15. The absorption coefficient up to about $f=3000 \mathrm{~Hz}$ is predicted extremely well by the model using the developed hybrid modelling approach. Also, the amplitude and frequency of the first peak in the absorption coefficient (at $f \approx 1155 \mathrm{~Hz}$ ) is similar to the measured absorption coefficient.

In Figure 15, the results are also compared to the microstructural model of Champoux and Stinson for cylindrical pores $[5,8]$ using the parameters in Table 3 . The flow resistivity $R_{s}$ is derived using the Kozeny-Carman formula:

$$
R_{s}=\frac{2 \mu q^{2} s_{0}}{\left(\Omega\left(r_{t} / 2\right)^{2}\right)}=1659 \mathrm{kgs}^{-1} \mathrm{~m}^{-3}
$$

where $\mu$ is the dynamic viscosity, the other parameters are accordingly to Table 3 , see also [5]. The first 2 parameters in this table are derived directly from the structure of the spheres. The third parameter, the equivalent pore radius $\left(r_{t}\right)$, is estimated based on the geometry of the sphere structure. All three parameters are equal to the ones used in the hybrid modeling approach.

The other 4 parameters are adjustable parameters and are chosen such that the predicted absorption coefficient follows the curve of the measured absorption coefficient. These 4 adjustable parameters can be tuned based on measurements or estimated based on the geometry of the porous material. The influence of the flow resistivity on the absorption coefficient is shown by varying the (steady) flow shape factor $s_{0}$, also shown in Figure 15. Note that the flow resistivity is the only parameter which depends on this flow shape factor. For a larger flow shape factor, and thus a larger flow resistivity, the absorption coefficient increases. Also, it can be seen that the frequency of the peaks in the sound absorption coefficient shifts to a lower frequency for a larger flow resistivity. This illustrates that the adjustable parameters introduce additional uncertainties when determining the absorption coefficient. Therefore, if a different porous material is chosen, or the structure of the porous material is varied, these parameters have to be validated again. Note that these adjustable parameters are not needed for the hybrid modeling approach, since the geometry of the porous material is used directly to find the sound field and the absorption coefficient. This makes the hybrid modeling approach more suitable as a design tool to study the influence of various (structural) properties of the porous material, like the size of the spheres or the stacking of multiple porous structures.

\begin{tabular}{lcc}
\hline Parameter & & \\
\hline Layer thickness & $H_{p}$ & $0.06 \mathrm{~m}$ \\
Porosity & $\Omega$ & $40 \%$ \\
Equivalent pore radius & $r_{t}$ & $0.7 \mathrm{~mm}$ \\
Tortuosity & $q^{2}$ & 1.9 \\
(Steady) flow shape factor & $s_{0}$ & 1 \\
Viscous shape factor & $s_{\rho}$ & 1 \\
Thermal shape factor & $s_{k}$ & 1 \\
\hline
\end{tabular}

Table 3: Parameters used in Champoux and Stinson model

\subsubsection{Sensitivity to porosity}

As illustrated in Figure 9, it is assumed that the encountered meshing problems are caused 
by the discretization of the geometry of the spheres. The problems were avoided by introducing a small spacing or overlap between the spheres, $\delta$. This spacing or overlap has as drawback that the number of spheres and thus the porosity of the sphere stacking in the model changes. This is illustrated in Figure 16 for: (left) spheres with negative spacing - an overlap of the spheres and (right) positive spacing - a gap between the spheres.
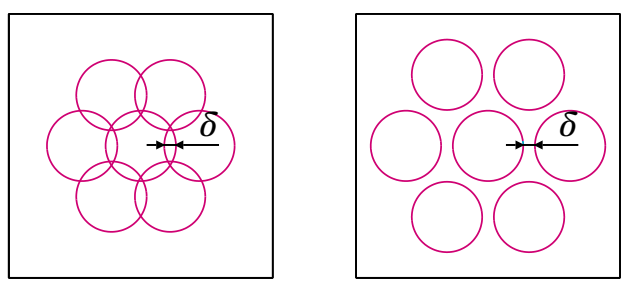

Figure 16: Overlap between spheres, $\delta<0$ (left). Spacing between spheres, $\delta>0$ (right).

When modeling a positive spacing $(\delta>0)$ between the spheres, the total number of spheres which fit within the viscous layer decreases and the porosity increases. Therefore, the maximum absorption coefficient is expected to decrease as well, since the porosity is higher than the optimal porosity. For models with a negative spacing $(\delta<0)$ it is possible to obtain the same number of spheres as marbles in the experiment. The number of spheres and marbles should agree for all models with a negative spacing. However, a difference between the number of spheres in the model and marbles in the measurements is observed. It is assumed that this is caused by differences in the sphere stacking. Note that also the layer height is slightly different.

The influence of this spacing on the sound absorption coefficient is shown in Figure 17. The parameters of the various models are listed in Table 4 , where $H_{p}$ is the height of the structure, $N$ is the number of marbles or spheres and $\Omega$ is the porosity.

Figure 17 shows that the predicted sound absorption coefficients have similar curves as the measured sound absorption coefficient. The frequency of the first peak in the absorption

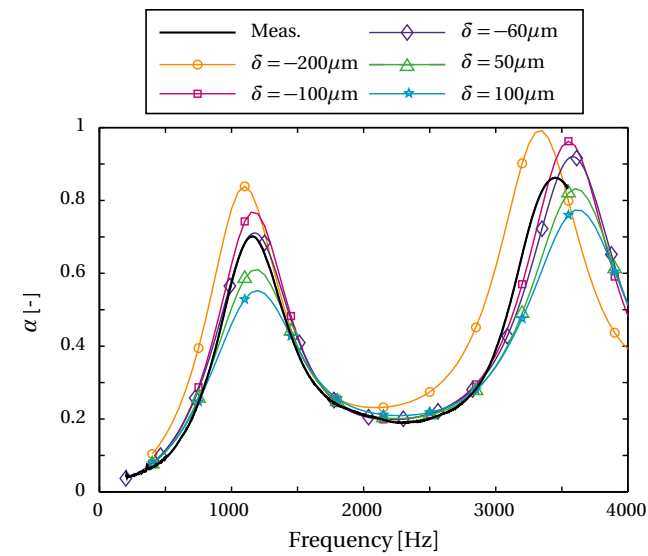

Figure 17: Sound absorption coefficient for variation in spacing between spheres.

coefficient $(f \approx 1155 \mathrm{~Hz})$ is predicted fairly well by the models. The sound absorption is best predicted by the models where the porosity and height of the structure are closest to the measurements. The best results are obtained with the model with a negative spacing of $\delta=-60 \mu \mathrm{m}$. This model predicts an absorption coefficient of $\alpha \approx 0.71$ at $f \approx 1160 \mathrm{~Hz}$, while the measured value is $\alpha \approx 0.70$ at $f \approx$ $1155 \mathrm{~Hz}$.

As expected, the amplitude of the absorption peak varies for the models. However, the behavior of the sound absorption for the frequencies up to approximately $3000 \mathrm{~Hz}$ is predicted very well. The minimum value of the absorption coefficient and the slope of the curve corresponds for most models. A difference can be seen above $f=3000 \mathrm{~Hz}$, where the frequency of the second absorption peak is predicted at a slightly higher frequency by most models. The largest differences are found for the model with $\delta=$ $-200 \mu \mathrm{m}$; the absorption coefficient is overestimated for a large part of the frequency range. However, the frequency of the second absorption peak is predicted better.

\subsubsection{Sensitivity to tube radius}

The viscothermal properties of medium II are derived using the low reduced frequency model, 


\begin{tabular}{lccc}
\hline Name & $H_{p}[\mathrm{~mm}]$ & $\mathrm{N}[-]$ & $\Omega[\%]$ \\
\hline Measurement & $\approx 61$ & $\approx 636$ & $\approx 40$ \\
Sim.: $\delta=-200 \mu \mathrm{m}$ & 57 & 636 & 38 \\
Sim.: $\delta=-100 \mu \mathrm{m}$ & 59 & 624 & 39 \\
Sim.: $\delta=-60 \mu \mathrm{m}$ & 60 & 606 & 41 \\
Sim.: $\delta=50 \mu \mathrm{m}$ & 62 & 576 & 46 \\
Sim.: $\delta=100 \mu \mathrm{m}$ & 63 & 540 & 50 \\
\hline
\end{tabular}

Table 4: Parameters of models for variation in spacing between spheres

discussed in Appendix A. As stated in Section 2.2, the variable which determines the properties of the viscous layer is the hydraulic radius of the characteristic tube $\left(r_{t}\right)$.

Since this value is an estimation of the true pore dimensions, a scaling factor is introduced:

$$
r_{\text {eff }}=\tau r_{t}
$$

where $\tau$ can be seen as a shape factor to estimate the effective radius of the tube $r_{\text {eff }}$. The influence of this shape factor is determined with a sensitivity study.

The shape factor is varied between $\tau=0.25$ and $\tau=1.25$ and the predicted sound absorption is compared with the measured absorption coefficient, as shown in Figure 18. All other parameters and the structure of spheres in the threedimensional model are kept constant. In these models a spacing of $\delta=-60 \mu \mathrm{m}$ is used, which means that for $\tau=1.00$, the model is exactly the same as the model 'Sim.: $\delta=-60 \mu \mathrm{m}$ ' in Section 3.1.2.

In Figure 18, it can be seen that when decreasing the value of the shape factor more sound is absorbed; this corresponds with a decreasing effective tube radius and therefore increasing viscothermal effects. For frequencies up to $3000 \mathrm{~Hz}$, the model with $\tau=1.00$, gives the best prediction of the absorption coefficient. The frequency of second peak in the sound absorption is predicted more accurately by the model with $\tau=0.50$. However, the amplitude of this second peak is predicted better by the model with $\tau=1.25$.

It could be possible to improve the prediction of the absorption coefficient when the spacing

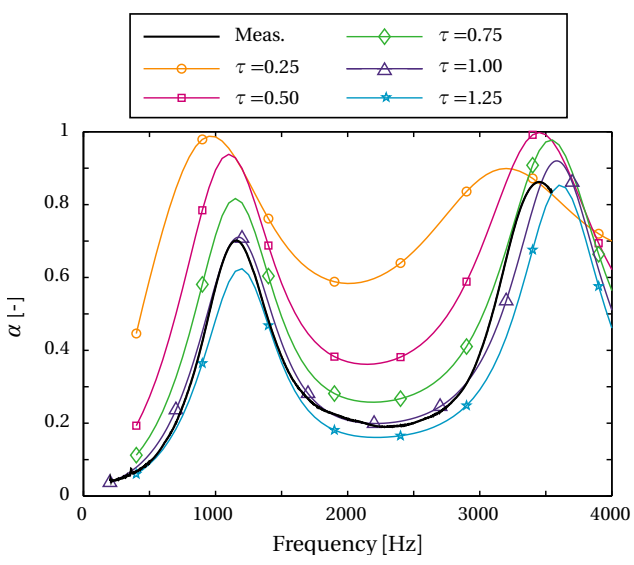

Figure 18: Sound absorption coefficient for variation in of $\tau=r_{\text {eff }} / r_{t}$.

is chosen such that the porosity is lower and the shape factor is chosen such that the viscous effects are smaller, for example: $\delta=-100 \mu \mathrm{m}$ and $\tau=1.25$. However, finding an optimal combination of spacing and shape factor is not researched yet.

\subsection{Influence of marble size and num- ber of layers}

In this section, the sound absorption coefficient for various structures of stacked marbles is investigated. Two sizes of marbles are used: (a) marbles with a diameter of $6 \mathrm{~mm}$ and (b) marbles with a diameter of $12 \mathrm{~mm}$. The parameters for the measurements and models are listed in Table 5 and 6 for $6 \mathrm{~mm}$ and $12 \mathrm{~mm}$ marbles, respectively. 


\subsubsection{Validation using $6 \mathrm{~mm}$ marbles}

Figure 19 and 20 show the absorption coefficient for structures with $n$ layers of stacked marbles with a diameter of $D=6 \mathrm{~mm}$. The lines with the colored markers indicate the measured sound absorption coefficient, which is again the average of 4 measurements. The lines with the open markers indicate the predicted sound absorption coefficient for a similar structure of spheres. All models are for $\delta=-60 \mu \mathrm{m}$ and $\tau=1.00$.

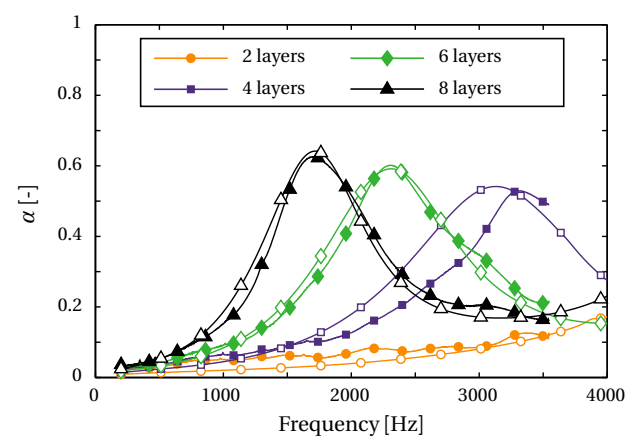

Figure 19: Measured sound absorption coefficient for stacked marbles (colored markers) and for predicted sound absorption coefficient (open markers) for $D=$ $6 \mathrm{~mm}$.

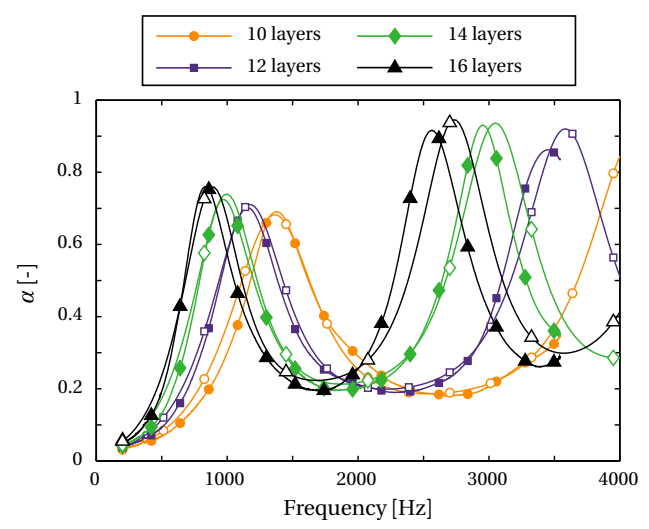

Figure 20: Measured sound absorption coefficient for stacked marbles (colored markers) and for predicted sound absorption coefficient (open markers) for $D=$ $6 \mathrm{~mm}$.

The predicted sound absorption coefficient for $6 \mathrm{~mm}$ marbles up to the second absorption peak corresponds extremely well with the measurement results, despite the differences in the sphere packing in the model and the packing of the marbles in the measurements. Only the absorption coefficient for the model with 4 layers is predicted less well. Also, Figure 20 shows that the frequencies of the second absorption peak are predicted at a higher frequency, compared to the measurements. It is expected that the predictions of the absorption coefficient will improve when the sphere packing in the model is more similar to the packing of marbles seen in the measurements, especially when the number of marbles and layer height correspond better to the packing in the measurements.

\subsubsection{Validation using $12 \mathrm{~mm}$ marbles}

The sound absorption coefficient for marbles with a diameter of $12 \mathrm{~mm}$ is shown in Figure 21. Again, the lines with the colored markers indicate the measured sound absorption coefficient and the lines with the open markers the predicted sound absorption coefficient.

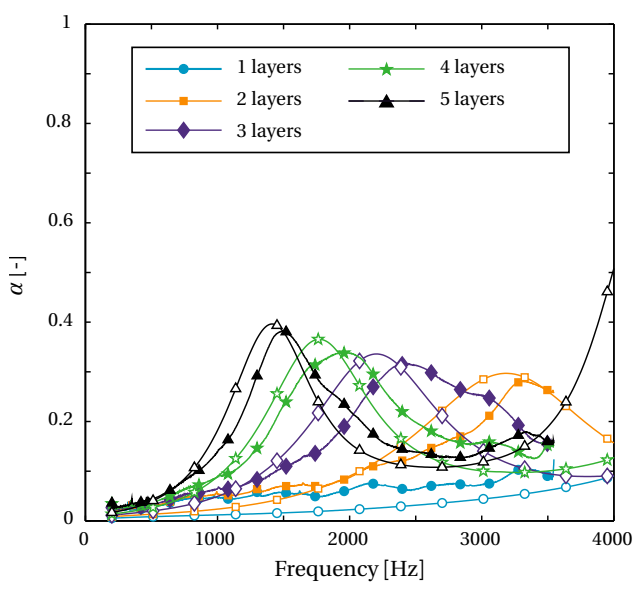

Figure 21: Measured sound absorption coefficient for stacked marbles (colored markers) and for predicted sound absorption coefficient (open markers) for $D=$ $12 \mathrm{~mm}$.

The differences between the predicted and measured absorption coefficient are larger than for the $6 \mathrm{~mm}$ marbles, while the number of mar- 


\begin{tabular}{ccccc}
\hline Layers & $\begin{array}{c}H_{p}[\mathrm{~mm}] \\
\text { Meas. }\end{array}$ & $\begin{array}{c}H_{p}[\mathrm{~mm}] \\
\text { Sim. }\end{array}$ & $\begin{array}{c}\mathrm{N} \\
\text { Meas. }\end{array}$ & $\begin{array}{c}\mathrm{N} \\
\text { Sim. }\end{array}$ \\
\hline 2 & $\approx 12$ & 12 & 106 & 101 \\
4 & $\approx 21$ & 22 & $\approx 212$ & 202 \\
6 & $\approx 30$ & 31 & $\approx 318$ & 303 \\
8 & $\approx 41$ & 41 & $\approx 424$ & 404 \\
10 & $\approx 51$ & 51 & $\approx 530$ & 505 \\
12 & $\approx 61$ & 60 & $\approx 636$ & 606 \\
14 & $\approx 71$ & 70 & $\approx 742$ & 707 \\
16 & $\approx 81$ & 79 & $\approx 848$ & 808 \\
\hline
\end{tabular}

Table 5: Parameters for $D=6 \mathrm{~mm}$

\begin{tabular}{ccccc}
\hline Layers & $\begin{array}{c}H_{p}[\mathrm{~mm}] \\
\text { Meas. }\end{array}$ & $\begin{array}{c}H_{p}[\mathrm{~mm}] \\
\text { Sim. }\end{array}$ & $\begin{array}{c}\mathrm{N} \\
\text { Meas. }\end{array}$ & $\begin{array}{c}\mathrm{N} \\
\text { Sim. }\end{array}$ \\
\hline 1 & $\approx 612$ & 13 & 12 & 12 \\
2 & $\approx 619$ & 23 & 24 & 24 \\
3 & $\approx 631$ & 33 & 36 & 36 \\
4 & $\approx 643$ & 42 & 48 & 48 \\
5 & $\approx 654$ & 52 & 60 & 60 \\
\hline
\end{tabular}

Table 6: Parameters for $D=12 \mathrm{~mm}$

bles and spheres in the packing does correspond exactly. The model results show that the frequency of the absorption peak is shifted to a lower frequency compared to the frequency of the measured absorption peaks. Also, the amplitude of the peaks is slightly larger for the predicted absorption coefficient. However, the predicted absorption coefficient shows the same behavior as the measured absorption coefficient.

\section{Conclusions and recommenda- tions}

The objective of this study was to develop a model to predict the sound absorption coefficient including both viscothermal effects and scattering effects. The modeling approach presented in this paper combines these elements. The model is validated for one-dimensional plane waves with impedance tube measurements of glass marbles and the model results closely match the measurement results.

The presented modeling approach can be used to find the absorption coefficient for existing porous asphalt roads. Furthermore, the model can be used in the process to design and manufacture new porous asphalt concretes, since the influence of individual design parameters - like stone size, stone shape, layer height and even multiple layers with different gradings - can be found directly.

In the future, the focus will be on closing the gap between the sphere structures used in the model and the stone structure in porous asphalt concrete, such that this modeling approach can be used as design tool in the development of new porous road surfaces. Also, future research will be done on the implementation and validation for oblique incidence, using threedimensional plane wave fields. 


\section{Acknowledgements}

This project is carried out in the framework of the innovation program 'GO Gebundelde Innovatiekracht', funded by the 'European Regional Development Fund', 'Regio Twente' and 'Provincie Overijssel'. The project partners are Apollo Tyres Global R\&D, University of Twente (Tire-Road Consortium), Reef Infra, STEMMER IMAGING and the Provincie Gelderland, and their support is gratefully acknowledged.

\section{Appendix A: Low reduced frequency model}

The properties of the viscous air layer are determined using the low reduced frequency (LRF) model, as described in Section 2.2.2. The LRF approach is described in detail by $[6,13,21,23]$. The key dimensionless parameters used in the LRF model are listed below:

- shear wave number:

$$
s(\omega)=r_{\mathrm{t}} \sqrt{\frac{\rho_{0} \omega}{\mu}}
$$

- ratio of specific heats:

$$
\gamma=\frac{C_{p}}{C_{v}}
$$

- Prandtl number:

$$
N_{\mathrm{pr}}=\frac{v}{\alpha_{t}}=\frac{\mu C_{p}}{\kappa}
$$

The shear wave number $s$ represents the ratio between the inertial and viscous forces, where $\mu$ is the dynamic viscosity and $r_{\mathrm{t}}$ is the characteristic radius of the tube. The ratio of specific heats is the ratio between the specific heat at constant pressure $C_{p}$ and at constant volume $C_{\nu}$. The Prandtl number is defined as the ratio between the viscous diffusion rate (denoted by the kinematic viscosity $v=\mu / \rho_{0}$ ) and the thermal diffusion rate (denoted by the thermal diffusivity $\alpha_{t}=\kappa /\left(\rho_{0} C_{p}\right)$, where $\kappa$ is the thermal conductivity).
The sound pressure $p(\omega, z)$ is given by:

$$
p(\omega, z)=A e^{\Gamma(\omega) k z}+B e^{-\Gamma(\omega) k z}
$$

The solution for the velocity in axial direction, averaged over the cross-section of the tube, yields:

$$
\bar{v}(\omega, z)=\frac{G(\omega)}{\rho_{0} c_{0}}\left[A e^{\Gamma(\omega) k z}-B e^{-\Gamma(\omega) k z}\right]
$$

where $A$ and $B$ are the complex amplitudes of the incident and reflected waves, $\rho_{0}, c_{0}$ and $k$ are for standard air conditions and the coefficients $\Gamma$ and $G$ depend on the angular frequency $\omega$ as well as the geometry of the cross-section. Here, only the solution for a cylindrical crosssection is given:

$$
\begin{aligned}
\Gamma(\omega) & =\sqrt{\frac{J_{0}(i \sqrt{i} s(\omega))}{J_{2}(i \sqrt{i} s(\omega))} \frac{\gamma}{n}} \\
G(\omega) & =-\frac{i}{\Gamma(\omega)} \frac{\gamma}{n}
\end{aligned}
$$

where $J_{0}$ and $J_{2}$ are Bessels functions of the first kind of order 0 and 2. The coefficient $n$ is given by:

$$
n(\omega)=\left[1+\frac{\gamma-1}{\gamma} \frac{J_{2}\left(i \sqrt{i N_{\mathrm{pr}}} s(\omega)\right)}{J_{0}\left(i \sqrt{i N_{\mathrm{pr}}} s(\omega)\right)}\right]^{-1}
$$

Using the low reduced frequency description for medium II, the wave number of this medium can be derived from the viscothermal wave propagation coefficient $\Gamma$ :

$$
k_{\mathrm{II}}(\omega)=-i k \Gamma(\omega)
$$

The particle velocity in medium II can also be expressed similar to Equation 3:

$$
v_{\mathrm{II}, n}(\omega, z)=\frac{-1}{Z_{\mathrm{II}(\omega)}}\left[A_{\mathrm{II}} e^{\Gamma(\omega) k z}-B_{\mathrm{II}} e^{-\Gamma(\omega) k z}\right]
$$

where $Z_{\mathrm{II}}$ is the characteristic impedance of medium II:

$$
Z_{\mathrm{II}}(\omega)=-\frac{\rho_{0} c_{0}}{G(\omega)}
$$

The (frequency dependent) speed of sound in medium II is determined by:

$$
c_{\mathrm{II}}(\omega)=\omega / k_{\mathrm{II}}(\omega)
$$


For the model described in Section 2.3, the ratio between the speed of sound in medium I and II, $c_{\text {II }}(\omega) / c_{0}$, as function of the frequency, is shown in Figure 22.

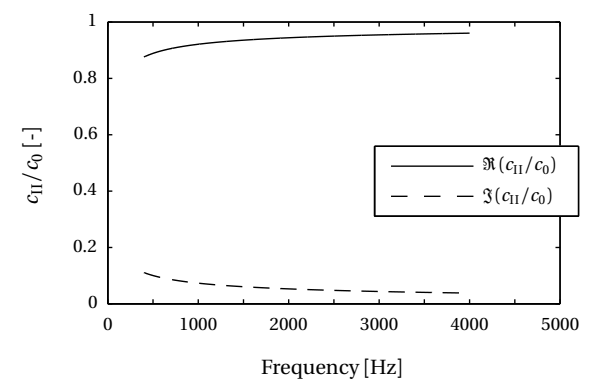

Figure 22: Ratio between speed of sound for the example model: $c_{\mathrm{II}}(\omega) / c_{0}$, real (-) and imaginary (- - -) part.

\section{Appendix B: Impedance tube mea- surements}

The impedance tube setup is shown in Figure 14. The important dimensions are summarized in Table 7 .

The measured microphone signals are controlled and processed using LabView and the post processing is performed in Matlab. The signals are analysed in the frequency domain using a method based on the method described by [15]. In this paper we focus on obtaining the absorption coefficient for normal incidence, using the sound pressure from all $4 \mathrm{mi}-$ crophones.

The sound pressure for a local plane wave is given by Equation 2, where the microphone location can be denoted by $z_{i}$. The microphone locations are known (listed in Table 7) and plane waves normal to the measurement plane are assumed. Therefore, the measured sound pressure for each microphone can be described by Equation 2 and written in matrix form:

$$
\left\{\begin{array}{c}
P_{1}\left(z_{1}, \omega\right) \\
P_{2}\left(z_{2}, \omega\right) \\
\vdots \\
P_{n}\left(z_{n}, \omega\right)
\end{array}\right\}=\left[\begin{array}{cc}
e^{i k z_{1}} & e^{-i k z_{1}} \\
e^{i k z_{2}} & e^{-i k z_{2}} \\
\vdots & \vdots \\
e^{i k z_{n}} & e^{-i k z_{n}}
\end{array}\right]\left\{\begin{array}{l}
A(\omega) \\
B(\omega)\}
\end{array}\right.
$$

where $\omega$ is the angular frequency. This system can be written as:

$$
\mathbf{P}=\mathbf{S a}
$$

The vector $\mathbf{P}$ contains the sound pressure measured by the microphones and the vector a contains the unknown complex amplitudes, $A(\omega)$ and $B(\omega)$. The matrix [S] describes the sound field in the impedance tube at the locations of the microphones. The system is solved for the complex amplitudes, in a least square sense using the normal equations:

$$
\mathbf{a}=\left(\mathbf{S}^{T} \mathbf{S}\right)^{-1} \mathbf{S}^{T} \mathbf{P}
$$

where $\mathbf{S}^{T}$ is the conjugate transpose of S.

The measured sound absorption coefficient $\alpha$ is then given by

$$
\alpha(\omega)=1-|R(\omega)|^{2}=1-\frac{B(\omega) \overline{B(\omega)}}{A(\omega) \overline{A(\omega)}}
$$

where $\overline{A(\omega)}$ and $\overline{B(\omega)}$ are the complex conjugates of the complex amplitudes, $A(\omega)$ and $B(\omega)$ respectively.

\section{References}

[1] J. Allard and N. Atalla. Propagation of Sound in Porous Media: Modelling Sound Absorbing Materials 2e. John Wiley \& Sons, 2009.

[2] F. Anfosso-Lédée and P. Dangla. Sound propagation above a porous road surface by boundary element method. Road Materials and Pavement Design, 7(3):289-312, 2006.

[3] F. Anfosso-Lédée, P. Dangla, and M. Bérengier. Sound propagation above a porous road surface with extended reaction by boundary element method. $J$. Acoust. Soc. Am., 122:731-736, 2007.

[4] K. Attenborough, I. Bashir, and S. Taherzadeh. Outdoor ground impedance models. J. Acoust. Soc. Am., 129:2806-2819, 2011. 


\begin{tabular}{lcc}
\hline Parameter & & Value \\
\hline Inner radius tube [mm] & $R$ & 25 \\
Outer radius tube [mm] & $R_{o}$ & 35 \\
Microphone distance 1-1 [mm] & $z_{1}$ & 0 \\
Microphone distance 1-2 [mm] & $z_{2}$ & 24 \\
Microphone distance 1-3 [mm] & $z_{3}$ & 62 \\
Microphone distance 1-4 [mm] & $z_{4}$ & 86 \\
Cutoff frequency [Hz] & $f_{\text {co }}$ & $c_{0} / 4 R$ \\
\hline
\end{tabular}

Table 7: Dimensions of impedance tube

[5] K. Attenborough, K. M. Li, and K. Horoshenkov. Predicting outdoor sound. Taylor \& Francis, 2007.

[6] W. M. Beltman. Viscothermal wave propagation including acousto-elastic interaction. PhD thesis, University of Twente, 1998.

[7] J.-P. Berenger. A perfectly matched layer for the absorption of electromagnetic waves. $J$. Comput. Phys., 114(2):185-200, 1994.

[8] M. C. Berengier, M. R. Stinson, G. A. Daigle, and J. F. Hamet. Porous road pavements: Acoustical characterization and propagation effects. J. Acoust. Soc. Am., 101(1):155162, 1997.

[9] M. Bezemer-Krijnen, Y. H. Wijnant, and A. de Boer. Three-dimensional modelling of sound absorption in porous asphalt pavement for oblique incident waves. In Euronoise 2015, 2015.

[10] D. T. Blackstock. Fundamentals of physical acoustics. John Wiley \& Sons, 2000.

[11] M. E. Delany and E. N. Bazley. Acoustical properties of fibrous absorbent materials. Appl. Acoust., 3(2):105-116, 1970.

[12] J. F. Hamet and M. Berengier. Acoustical characteristics of porous pavements: a new phenomenological model. In INTERNOISE 93: people versus noise, Leuven, Belgium, pages $641-646,1993$.
[13] M. H. C. Hannink. Acoustic resonators for the reduction of sound radiation and transmission. PhD thesis, University of Twente, 2007.

[14] M. H. C. Hannink., Y. H. Wijnant, and A. de Boer. Optimised sound absorbing trim panels for the reduction of aircraft cabin noise. In Eleventh International Congress on Sound and Vibration, ICVS 11, 2004.

[15] E. R. Kuipers, Y. H. Wijnant, and A. de Boer. Measuring oblique incidence sound absorption using a local plane wave assumption. Acta Acustica united with Acustica, 100(2):205-214, 2014.

[16] C.-Y. Lee, M. J. Leamy, and J. H. Nadler. Acoustic absorption calculation in irreducible porous media: A unified computational approach. J. Acoust. Soc. Am., 126(4):1862-1870, 2009.

[17] F. P. Mechel. Sound fields at periodic absorbers. J. Sound Vib., 136(3):379-412, 1990.

[18] M. Ochmann and H. Brick. Acoustical radiation and scattering above an impedance plane. In Computational Acoustics of Noise Propagation in Fluids-Finite and Boundary Element Methods, pages 459494. Springer, 2008.

[19] E. Sarradj. Multi-domain boundary element method for sound fields in and around porous absorbers. Acta Acustica united with Acustica, 89:21-27, 2003. 
[20] J. H. Schutte. Numerical Simulation of Tyre/Road Noise. PhD thesis, University of Twente, 2011.

[21] H. Tijdeman. On the propagation of sound waves in cylindrical tubes. J. Sound Vib., 39(1):1-33, 1975.

[22] O. Umnova, K. Attenborough, and K. M. Li. A cell model for the acoustical properties of packings of spheres. Acta Acustica united with Acustica, 87(2):226-235, 2001.

[23] F. J. M. van der Eerden. Noise reduction with coupled prismatic tubes. $\mathrm{PhD}$ thesis, University of Twente, 2000.

[24] Y. H Wijnant, E. R. Kuipers, and A. de Boer. Development and application of a new method for the insitu measurement of sound absorption. In Proceeding of ISMA2010 including USD2010, 2010.

[25] T. G. Zielinski. Microstructure-based calculations and experimental results for sound absorbing porous layers of randomly packed rigid spherical beads (034905). J. Appl. Phys., 116(3), 2014. 


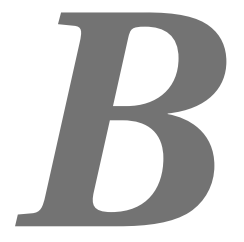

\section{Prediction of sound absorption of stacked granular materials for normal and oblique incident sound waves}

This chapter is based on the paper 'Prediction of Sound Absorption of Stacked Granular Materials for Normal and Oblique Incident Sound Waves', [19], published in: 'Acta Acustica united with Acustica', Vol. 104 (2018) 464 476. Copyright by (c)(2018) S. Hirzel Verlag/European Acoustics Association, http//dx.doi.org/10.3813/AAA.919189. Readers must contact the publisher for reprint or permission to use the material in any form. 


\title{
Prediction of sound absorption of stacked granular materials for normal and oblique incident sound waves
}

\author{
M. Bezemer-Krijnen ${ }^{1)}$, Y.H. Wijnant ${ }^{1)}$, A. de Boer ${ }^{1)}$ \\ Acta Acustica united with Acustica, Volume 104, Number 3, May/June 2018 \\ 1) Faculty of Engineering Technology, University of Twente, P.O. Box 217, 7500 AE Enschede, \\ The Netherlands. \\ m.bezemer@utwente.nl
}

\begin{abstract}
Tire/road noise is a problem in many (densely) populated areas. It can be significantly reduced by using porous asphalt concrete. A challenge is to develop porous asphalt concrete, such that the most dominant frequencies in tire/road noise will be absorbed by the road surface. It is especially important to also reduce and absorb oblique incident sound waves, since tires radiate noise normal to the tire surface, which means oblique incident waves on the road surface. Predicting the behavior of porous asphalt concrete using models is complex, especially when non-local effects and scattering effects are included. The objective of this paper is to show a modeling approach to predict sound absorption for oblique incident waves in three-dimensional porous materials. Using this method, one is able to predict the sound absorption of porous road surfaces in the design phase. This modeling approach includes a two-step approach in which first the viscothermal energy dissipation inside the pores between the rigid materials (stones) are estimated and then, secondly, the non-local effects such as scattering on the stones within the porous road surface are computed using a finite element model. The combination of both sound fields gives the total sound field in and above the three-dimensional porous material, which is used to determine the sound absorption coefficient. The analytical viscothermal and scattering solution are discussed in this paper and the modeling approach is validated with experiments using a box with stacked marbles for several angles of incidence.
\end{abstract}




\section{Introduction}

Tire/road noise is a complex noise problem, partly because the noise generation depends on many different variables and partly because it is very difficult to insulate the sound sources itself. However, it is possible to reduce the noise by avoiding or minimising the sound excitation mechanisms of tire/road noise. For instance by designing a silent tire - by optimising the tread design, changing the stiffness properties of the tire or by influencing the tire resonance frequencies - or by changing the road roughness properties.

It is also possible to address the area where tire/road noise leads to problems. Typical measures to reduce traffic noise are noise barriers or porous road surfaces. To predict the effect of such measures, modeling techniques are widely used. These vary from (simplified) global methods where complete areas are considered to detailed models of the measure itself. In [2] an overview is given of several models used to predict the impedance and sound absorption of porous asphalt concrete. One category of these models are the microstructural models, which are based on analytical descriptions of the (simplified) microstructure of the porous material, such as pores filled with air in an elastic or rigid frame. The pressure and velocity inside these pores are described analytically and both the thermal and the viscous dissipation of energy are included. Again, there are many models available to describe such sound absorbing materials. One of the first models describing the thermal and viscous effects for cylindrical tubes was given by Zwikker and Kosten [20], and was further validated and described by [15, 17]. In [15] and in [16], the theory is expanded for arbitrary cross-sections, using shape factors. To find the properties of the bulk material, it is common to use the Biot theory for fluidsaturated porous materials with a rigid or elastic frame [7, 8]. In [1, 5, 3] some of the well-known models are compared.

\subsection{Objective}

The work described in this paper is part of a larger research to develop a modeling approach to predict the sound absorption coefficient for porous asphalt roads in the design phase. This paper covers the first steps of this modeling approach: the development and validation of a hybrid analytical/numerical model to predict the sound absorption coefficient for oblique incident waves on a three-dimensional porous or granular structure. An important and distinctive property of this modeling approach is that the local behavior of the porous surface is included - like scattering and interconnected pores - such that the sound absorption coefficient for oblique incident sound waves can be predicted. We have chosen to first model a granular structure, since such a structure is welldefined and the model validation is more controllable and repeatable than that of true porous asphalt concrete.

In most of the existing modeling approaches the porous road surface is considered as a homogeneous, non-locally reacting medium, meaning that the acoustic response of a point on the surface does not depend on the location on that surface or on the angle of incidence of the incident sound waves. When a microstructural model is used to find the acoustic properties of a single pore within a flexible or rigid bulk material, these properties are then generalised to describe the properties of the entire material, for example by including the porosity or applying shape factors. Therefore, the properties of the individual pores are averaged and the material is assumed to be homogeneous and non-locally reacting. Furthermore, the behavior for oblique incidence is fixed.

However, rolling tires will radiate noise in all directions and the influence of these oblique, and mostly grazing, sound waves should therefore be included in the predictive models. In some cases, it is possible to achieve this completely analytically (as shown in [14]). In other cases the analytical equations to describe the surface structure become increasingly complicated and other methods are preferred. In this paper, we describe a newly developed hybrid analyti- 
cal/numerical approach. In this approach, we combine the analytical description of an undisturbed background sound field, including a viscous medium, with a finite element model to include the scattering of the stones of the porous asphalt concrete. The main reason this modeling approach is developed is to keep the computational time low, since applying this approach only a local scattering problem has to be solved and the viscothermal behavior is included analytically, such that no boundary layers are required in the finite element model. In this paper, for modeling purposes, we consider the porous asphalt concrete as a porous (granular) material; in which the stones and bitumen are considered as rigid stacked spheres and the connected spaces in between the spheres are considered as pores filled with (viscous) air. The properties of the viscous medium are based on the behavior of the air inside a single pore, including viscothermal effects, and is described with the so-called low reduced frequency (LRF) model, $[4,10,17,18]$. Note that other models to describe the viscothermal properties of the air inside the porous material can be used as well. In this implementation, we have chosen the LRF model since this model approach provides analytical expressions for the sound pressure and particle velocity and since the parameters needed to describe the porous material can be found based only on the geometry of this material.

A similar approach and the resulting threedimensional model, as well as the validation for normal incidence using a Kundt tube, are described in [6]. The key characteristics and main assumptions of the modeling approach are repeated and the adjustments and extensions of the modeling approach for oblique incidence are highlighted in this paper. Moreover, in this paper, the focus is on the modeling approach for oblique incidence and on an experimental method to validate the modeling approach using a box filled with glass marbles.

\subsection{Outline}

A short discussion of the modeling approach and the resulting model is given in Section 2.
For a more detailed description of the modeling approach, the reader is referred to [6]. The model approach is validated for oblique incidence. The measurement setup, technique and the results are discussed in Section 3. As discussed, the porous asphalt concrete is simplified to a granular structure, allowing the modeling approach to be experimentally validated using a structure of stacked glass marbles. The model and measurement results for various angles of incidences are discussed in this section, underlining the importance of including the local surface behavior in the predictive models. The conclusions and recommendations are given in Section 4.

\section{Modeling approach and imple- mentation}

The developed modeling approach is based on the combination of the solutions of two subsystems. A schematic view of this modeling approach is given in Figure 1.

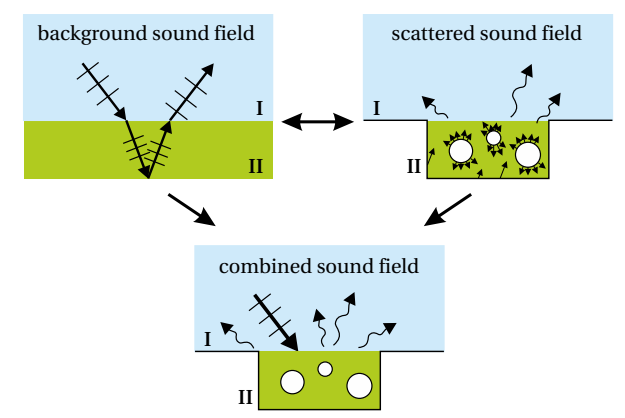

Figure 1: Schematic view of the modeling approach

The subsystems are based on a model consisting of two mediums: medium I is defined according to standard air conditions and medium II is modelled as a viscous air layer. The subsystem shown at the upper left corner of Figure 1 is called the background sound field and describes the wave propagation in these two mediums analytically. The properties of the viscous medium are defined using a microstructural model for a single pore. Note that in this subsystem, the granular structure, which represents the porous asphalt concrete, is not directly 
included in the viscous air layer. The given analytical solution is used as a background field undisturbed by the granular structure. However, the viscous properties are included in the second medium.

The second subsystem describes the scattering of the incident sound field on the porous asphalt concrete and is solved numerically as a scattering problem using a finite element method. In this subsystem a simplified, threedimensional model of the granular structure inside a rigid box is included in the viscous air layer and is modelled such that only the viscous air between the stones in this structure is considered. The coupling between the background sound field and the scattered sound field is realised numerically. The properties of the air layer and the viscous air layer are equal for both the background and scattering subsystem. The background and scattered fields are combined to find the total solution, which therefore includes both the viscothermal effects and the scattering effects. This is allowed, since both solutions satisfy the Helmholtz equation. The modeling approach is validated for normal incident plane waves, as discussed in [6]. Figure 2 shows the predicted and measured sound absorption coefficient for several structures of stacked spheres using the impedance tube technique.

\subsection{Description of sound field}

The solution of both the background sound field as well as the scattered sound field are based on the Helmholtz equation [9]:

$$
\nabla^{2} \Phi+k_{j}^{2} \Phi=0
$$

where $\nabla$ is the Laplace operator, $\Phi$ is the velocity potential and $k_{j}$ is the (complex) wave number of medium $j$. The wavenumber in medium $\mathrm{I}$, is $k_{\mathrm{I}}=k$; the wave number assuming standard air conditions, defined as $k=\omega / c_{0}$, where $\omega$ the angular frequency and $c_{0}$ the speed of sound in air. The wave number in medium $k_{\mathrm{II}}$, the viscous air layer, includes the viscothermal properties of this medium. In the implemented modeling approach, these properties are based on the low-reduced frequency (LRF) model (e.g.

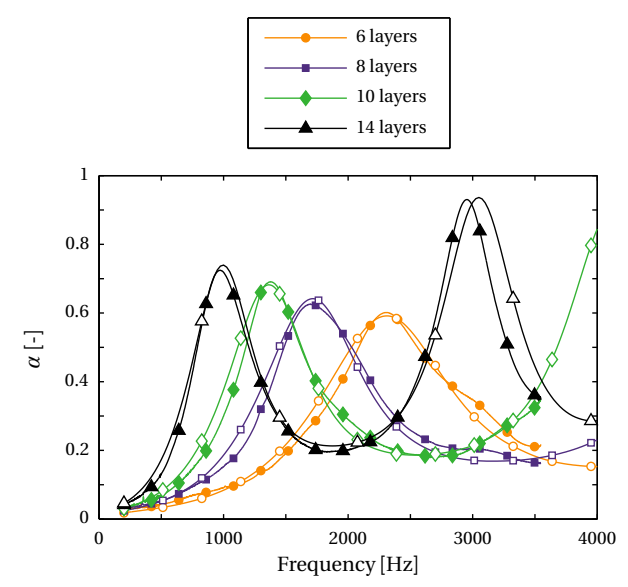

Figure 2: Validation of modeling approach for several structures of stacked spheres with $D=6 \mathrm{~mm}$, using Kundt tube. The measured absorption coefficient is indicated by the colored markers and the predicted absorption coefficient by the open markers [6].

$[4,10,17,18])$ and are summarized in Appendix A. Note that other microstructural model approaches can also be used to describe the properties of the viscous air layer.

The sound pressure field in medium $j$, indicated by $p_{j}$, for an incoming sound wave and reflected sound wave is described by:

$$
p_{j}=A_{j} e^{i k_{j} \xi(\mathbf{r}, \theta, \varphi)}+B_{j} e^{-i k_{j} \xi(\mathbf{r}, \theta, \varphi)}
$$

using the $e^{i \omega t}$ convention and where $A_{j}$ is the complex amplitude of the incoming sound waves, $B_{j}$ the complex amplitude of the reflected sound waves, both in medium $j$, and $\xi_{j}\left(\mathbf{r}, \theta_{j}, \varphi_{j}\right)$ is the spatial coordinate defined by:

$$
\begin{aligned}
\xi_{j}\left(\mathbf{r}, \theta_{j}, \varphi_{j}\right)= & x \sin \theta_{j} \cos \varphi_{j} \\
& +y \sin \theta_{j} \sin \varphi_{j}+z \cos \theta_{j}
\end{aligned}
$$

where $\mathbf{r}=\{x, y, z\}$ are the coordinates, $\theta_{j}$ the polar angle of incidence and $\varphi_{j}$ azimuthal angle of incidence (both for medium $j$ ), as shown in Figure 3. It is assumed that the polar angle of incidence $\theta_{j}=\theta_{j, \text { in }}$ is directed opposite to the angle of the reflected waves: $\theta_{j}=-\theta_{j \text {,refl }}$. The azimuthal angle is defined by $\varphi_{j}=\varphi_{j, \text { in }}=$ $\varphi_{j, \mathrm{refl}}$. 


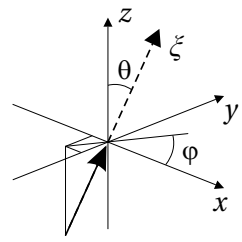

Figure 3: Conventions of direction, polar angle $\theta$ and azimuthal angle $\varphi$.

The background sound field is implemented in the numerical model according to Equation 2 assuming unit amplitude for all waves ( $A_{\mathrm{I}}=$ $B_{\mathrm{I}}=A_{\mathrm{II}}=B_{\mathrm{II}}=1$ ) and the boundary conditions shown in Figure 4. The scattering problem follows from the solution of the background field combined with the boundary conditions shown in Figure 5. This implementation of the background field and the scattered field ensures a local scattering problem. This is an important property of this modeling approach, because the model can be kept relatively small, such that the computational time is relatively small as well.

Note that, while the boundary conditions at the interface between medium I and medium II (as shown in Figure 4) are used to solve the background field, these are not required to solve the scattering problem. We chose to implement these boundary conditions, since it ensures that the scattering problem is local and that the solution of both subsystems, as well as the combined system, is continuous over the interface. Without these boundary conditions, the solution for the total combined system will still be continuous over the interface, but the solution for both subsystems will not be continuous over this interface.

For a more detailed description of the implementation of the sound field and the relations for the particle velocity, the reader is referred to [6]. Note that the focus of [6] is on normal incident sound waves.

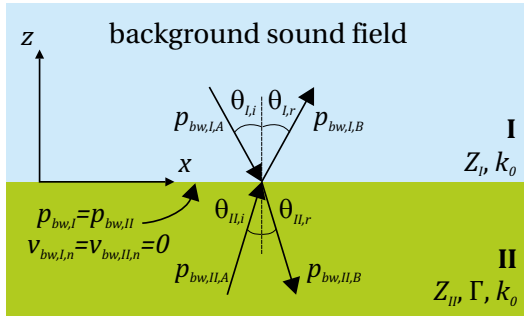

Figure 4: Overview of implementation and boundary conditions for the background field, where the index $b w$ indicates the background field.

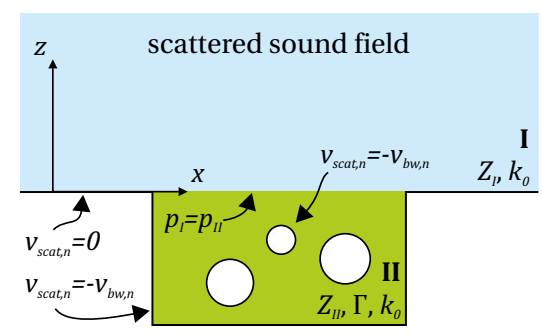

Figure 5: Overview of implementation and boundary conditions for the scattered field, where $v_{\text {scat }}$ is the particle velocity of the scattered field and $v_{\text {bw }}$ is the particle velocity of the background field.

\subsection{Implementation modeling approach}

The model described in this paper is based on the measurement setup used for the validation of the model, which is described in Section 3. The approach is validated for a rectangular box with sound hard boundaries filled with glass marbles with a diameter of $12 \mathrm{~mm}$. The box was placed in the center of a large rectangular wooden baffle, such that the top surface of the box and the baffle were aligned. The dimensions and important properties of the measurement setup and the structure of marbles are listed in Table 1 in Section 3.

The modeling approach with the two subsystems is implemented together as one numerical model and is solved for the combined sound field, using the multiphysics package COMSOL (version 4.4), in combination with Matlab (version 2013a). The problem is solved in the frequency domain using the MUltifrontal Massively Parallel sparse direct Solver (MUMPS); a 
stationary linear solver. To enhance the computational speed and accessible memory, the outof-core option is used.

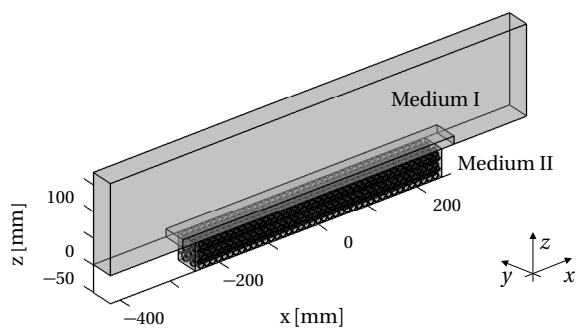

Figure 6: Geometry of the numerical model as implemented in COMSOL Multiphysics.

In Figure 6, the geometry of the numerical model is shown. Important properties of this model are:

- Medium I (air layer): the air layer is modelled as a rectangular domain. The boundaries of medium I are modeled such that there are no reflections at these boundaries.

- Medium II (viscous air layer): only the air between the stacked marbles (represented by spheres) inside a rectangular domain (representing the box) is modelled. The frequency dependent properties of this viscous air are based on the analytical description of a representative cylindrical tube using the LRF model, as discussed in [6].

- The background sound field is solved for a sound field undisturbed by the granular structure, according to Equation 2. The direction of the incoming and reflected waves is included in the spatial coordinate $\xi_{j}$. The boundary conditions are implemented as shown in Figure 4.

- The scattered sound field is solved numerically for the exact geometry, using the boundary conditions indicated in Figure 5 .

- The coupling between the two subsystems is provided by the boundary conditions for both subsystems and by the correspond- ing properties of the viscous air layer in medium II.

- The implemented background sound field is a solution of the Helmholtz equation and the solution of the scattering problem is also a solution of the Helmholtz equation. Furthermore, the solutions of both subsystems are linear and can therefore be added to find the total solution of the combined sound field. This total solution can be used to find the sound absorption coefficient of the porous granular structure.

Due to the dimensions of the measurement setup and the limitations of the used computer, some modeling concessions are made:

- Only a slice of the total box is modelled. This slice is aligned with the direction of the incident waves (the longitudinal direction) and the direction of azimuthal angle of incidence is fixed at $\varphi=0^{\circ}$, the direction along the $\mathrm{x}$-axis. The thickness of the modelled slice is $45 \mathrm{~mm}$, which is about 1.5 times the width of the unit cell. A result of this implementation is that the simulated problem is not entirely three-dimensional and effects caused by the width of the box and granular structure in the y-direction can be missed.

- To keep the number of meshing elements within the box with the spheres structure reasonable and to avoid meshing problems, the spheres are modelled with a small overlap of $\delta=80 \mu \mathrm{m}$, this is discussed in more detail in [6].

- The model is solved for a frequency range of $400-3000 \mathrm{~Hz}$, which is divided into three smaller ranges. The resulting geometry and mesh of the model depends on the frequency range: (1) the dimensions of the baffle are larger than the longest wavelength and (2) the mesh size is chosen such that the shortest wavelength is modelled with at least 10 elements.

The resulting three-dimensional model used for the validation of the modeling approach is quite large. For example, the numerical model used for the frequency range $1000-2000 \mathrm{~Hz}$ has 
$2.1 \cdot 10^{6}$ number of elements. However, for this type of problem, the developed hybrid modeling approach keeps the computational time relatively low. The first advantage of this approach is that the scattering problem that has to be solved is only a local scattering problem, because of the velocity boundary condition at the interface between medium I and II. Therefore, the dimensions of air surrounding the box with the granular structure can be kept small. Secondly, the viscothermal behavior is accounted for directly in the analytical description of the wave number in the numerical model, such that in the mesh of the air between the granular structure in medium II, no boundary layers are required which drastically reduces the number of elements and the computation time.

\subsection{Sound absorption coefficient}

The developed modeling approach can be used to predict the sound absorption coefficient of different structures of spheres for an arbitrary angle of incidence. Therefore, the modeling approach can be used as design tool to study, for example, the influence of the stone sizes in asphalt gradings and the effect of multiple layers with different gradings on the sound absorption for normal and oblique incident sound waves.

\subsubsection{Sound absorption coefficient in nu- merical model}

The sound absorption coefficient for the granular structure in medium II is derived from the model assuming local (specular) plane wave propagation in the air layer. The area averaged absorption coefficient is determined from the ratio between the time-averaged incident sound power $W_{\text {in }}$ and the time-averaged active sound power $W_{\text {ac }}$, both considered over an interface $S$, according to the local plane wave method, [19]:

$$
\alpha=\frac{W_{\mathrm{ac}}}{W_{\mathrm{in}}}=\frac{\int \mathbf{I}_{\mathrm{ac}} \cdot \mathbf{n} d S}{\int I_{\mathrm{in}} d S}
$$

The active and incident sound power are obtained from the integration of the corresponding intensities over the interface $S$, where $\mathbf{n}$ is the unit vector normal to this interface.

The active intensity follows directly from the solution of the numerical model:

$$
\mathbf{I}_{\mathrm{ac}}=\frac{1}{2} \operatorname{Re}(p \overline{\mathbf{v}})
$$

where $\overline{\mathbf{v}}$ denotes the complex conjugate of the total complex particle velocity $\mathbf{v}=\mathbf{v}_{\mathrm{bw}}+\mathbf{v}_{\text {scat }}$ and $p$ is the total complex sound pressure: $p=p_{\mathrm{bw}}+$ $p_{\text {scat }}$.

The normal component of the incident intensity can be derived using a local plane wave assumption at any point at the interface $S$ :

$$
I_{\text {in }, n}=\frac{A_{n} \overline{A_{n}}}{2 \rho_{0} c_{0}}
$$

where $\overline{A_{n}}$ denotes the complex conjugate of $A_{n}$, the normal component of the complex amplitude of the incident wave, with $A_{n}=$ $\frac{1}{2}\left(p-\rho_{0} c_{0} v_{n}\right)$ and $v_{n}$ is the normal component of the particle velocity. The surface normal of the horizontal plane between medium $\mathrm{I}$ and medium II is directed in positive $\mathrm{z}$ direction.

Due to this approach, only the normal components of the particle velocity at the interface $S$ are considered. This is according to the socalled local plane wave (LPW) method. For incident waves with larger angles of incidence this can lead to accuracy issues, since the normal component of the velocity $v_{n}$ is small compared to total particle velocity $\mathbf{v}$. For larger angles of incidence, it would be better to assume local specular plane waves, in which the angle of incidence is included.

\subsubsection{Measured area averaged sound absorp- tion coefficient}

The sound absorption coefficient in the measurement method is derived with another method, since the particle velocity is not measured directly. Therefore, we use the pressure, measured at $N$ different positions in a small cubic array, to derive the relations for the active 
sound field between these measured points, assuming local specular plane waves (LSPW). Two implementations of the LSPW method are possible, one where the angle of the incident and reflected wave are derived using an optimisation algorithm, or an implementation where the angle of the incident and reflected wave are estimated beforehand. Here, we implemented the latter: the angle of the incident and reflected wave are estimated beforehand, based on the measurement setup. The LSPW method is described in more detail in [12].

The active, complex pressure $\hat{p}_{q}$ is measured by microphone $q$ for microphone position $q=$ $1,2, \ldots, N$. At each microphone the pressure can be described by:

$$
\begin{aligned}
\hat{p}_{q}\left(\omega, \mathbf{r}_{q}\right)= & A(\omega) e^{i k_{\mathrm{I}}(\omega) \xi_{\mathrm{I}}\left(\mathbf{r}_{q}\right)} \\
& +B(\omega) e^{-i k_{\mathrm{I}}(\omega) \xi_{\mathrm{I}}\left(\mathbf{r}_{q}\right)}
\end{aligned}
$$

The spatial coordinate $\xi_{\mathrm{I}}\left(\mathbf{r}_{q}\right)$ is directed along the surface normal (Equation 3) and depends on the position $\mathbf{r}_{q}$ of the $q$ th microphone. The relations for $N$ microphones can be grouped in a set of equations:

$$
\hat{\mathbf{P}}=\mathbf{S a}
$$

where the vector $\hat{\mathbf{P}}$ contains the active sound pressures measured at $N$ microphones, the vector a contains the unknown complex amplitudes, $A(\omega)$ and $B(\omega)$ and the matrix $\mathbf{S}$ describes the sound field at the locations of the microphones for the active sound field.

The system is solved for the complex amplitudes in a least square sense using the normal equations:

$$
\mathbf{a}=\left(\mathbf{S}^{T} \mathbf{S}\right)^{-1} \mathbf{S}^{T} \mathbf{P}
$$

where $\mathbf{S}^{T}$ is the conjugate transpose of S.

For each measurement position, a so-called local sound absorption coefficient can be calculated based on the ratio of active and incident intensity, as a (local) approximation of the sound absorption coefficient defined in Equation 4 . Here, the local active intensity is found using the difference between the local incident and reflected intensity, in direction of the outward surface normal:

$$
\begin{aligned}
\mathbf{I}_{\mathrm{ac}, \text { local }}(\omega, \mathbf{r}) \cdot \mathbf{n}= & I_{\mathrm{in}, \text { local }, n}(\omega, \mathbf{r}) \\
& -I_{\text {refl, local }, n}(\omega, \mathbf{r})
\end{aligned}
$$

with:

$$
\begin{aligned}
I_{\text {in, local }, n}(\omega, \mathbf{r}) & =\frac{A(\omega) \overline{A(\omega)}}{2 \rho_{0} c_{0}} \cos \hat{\theta} \\
I_{\text {refl, local }, n}(\omega, \mathbf{r}) & =\frac{B(\omega) \overline{B(\omega)}}{2 \rho_{0} c_{0}} \cos \hat{\theta}
\end{aligned}
$$

where $\hat{\theta}$ is the estimated polar angle of incidence, corresponding with the angle between the incident sound waves and the outward surface normal.

For structures with local behavior, such as the porous (granular) structures treated in this paper, the local sound absorption coefficient varies over the measured surface. The variation depends on the amount of geometrical variation, the dimensions of the structure and the measurement position on this surface. Therefore, it is important to combine the results from multiple measurements to find the so-called area averaged absorption coefficient $\hat{\alpha}_{\text {area }}$, which is an approximation of the sound absorption coefficient defined in Equation 4:

$$
\hat{\alpha}_{\text {area }}=\frac{\sum_{m=1}^{M}\left(I_{\mathrm{in}, n, m}-I_{\text {refl }, n, m}\right) \Delta S_{m}}{\sum_{m=1}^{M} I_{\mathrm{in}, n, m} \Delta S_{m}}
$$

where $I_{\text {in }, n, m}=I_{\text {in, local }, n, m}, I_{\text {refl }, n, m}=I_{\text {refl, local }, n, m}$, $M$ is the total number of measurements and $\Delta S_{m}$ is the area covered by the $m$ th measurement. To approximate the area averaged sound absorption coefficient, the integral of the incident and active intensity over the surface $S$ is now replaced with the sum over a small area $\Delta S_{m}$, representative for the dimensions of the microphone array.

Note that it is important to measure an enclosed area above and around the surface of the material when evaluating the area-averaged absorption coefficient to minimize the leakage of energy. Otherwise energy will leak in direction parallel to the sound hard surfaces enclosing the measurement area since the micro- 
phones are always positioned at a certain distance above the measurement surface. Figure 7 illustrates the enclosed measurement box around the measurement surface. This is described in more detail by [11, p. 55-62].

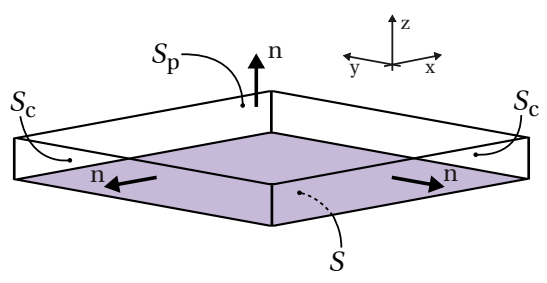

Figure 7: General view of measurement surface $S$ with the projected surface $S_{p}$ as well as the circumferential surfaces $S_{c}$ indicated. Together these surfaces form an enclosed measurement box around the measurement surface. The outward surface normal $\mathbf{n}$ of the surfaces is also indicated in the figure.

The intensity vectors shown in Figure 8 illustrate this as well. In this paper, the above described LSPW method is implemented for the surface parallel to the measurement surface, the so-called projected surface $S_{p}$, and the circumferential surfaces $S_{c}$. Alternatively, the LPW method can be implemented for the enclosing circumferential surfaces and the LSPW method for the projected surface. However, this shows only small differences in sound absorption coefficient.

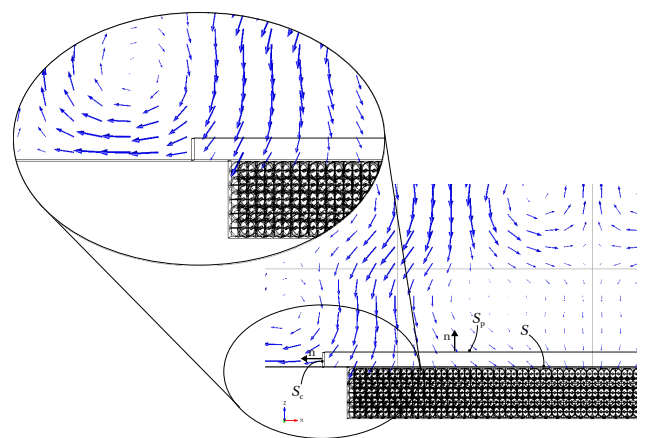

Figure 8: Intensity vectors above the measurement surface $S$, for $\theta=0^{\circ}$ and $f=1500 \mathrm{~Hz}$.

\section{Validation for normal and oblique incidence}

The developed modeling approach is validated using an in-situ measurement technique of the sound absorption coefficient for a structure of stacked glass marbles within a sound hard box, as shown in Figure 9, embedded in a sound hard baffle. The dimensions of this structure, box and baffle are listed in Table 1. The measurement setup is described in Section 3.1 and shown in Figure 10.

A structure with the same dimensions is modelled in the numerical model, as described in Section 2.2. The geometry of this model is shown in Figure 6.

The objective of these measurements is to show that the sound absorption coefficient is indeed dependent on the angle of incidence. Furthermore, the objective is to show that the hybrid modeling approach can be used to predict the sound absorption coefficient of complex structures, in this case a box filled with stacked glass marbles. The box with marbles is a well defined structure, and therefore very suitable to show that the developed hybrid modeling approach can be used to predict the sound absorption coefficient of complex structures. The final objective of this research is to model porous asphalt road surfaces such that the sound absorption coefficient can be optimised in the design phase. The used measurement technique is suitable for in-situ situations without destruction of the road surfaces. Furthermore, the measurement technique can be used for quality checks of large sections of road surfaces.

\subsection{Measurement setup and techniques}

The modeling approach is validated using a box filled with glass marbles. The walls of the box are made of $51 \mathrm{~mm}$ solid aluminum. The bottom of the box, an aluminium plate of $4 \mathrm{~mm}$ thickness, has been rigidly fixed to this structure with bolts. The box contains five layers with approximately 4878 marbles which have been stacked 


\begin{tabular}{lr}
\hline Parameter & Value \\
\hline Width box (inside) [mm] & 250 \\
Length box (inside) [mm] & 500 \\
Height box (inside) [mm] & 50 \\
Wall thickness [mm] & 51 \\
Thickness bottom plate [mm] & 4 \\
Width baffle [mm] & 1033 \\
Length baffle [mm] & 1031 \\
Diameter marbles [mm] & 12 \\
Number of marbles [-] & 4878 \\
Number of layers in z-dir. [-] & 5 \\
\hline
\end{tabular}

Table 1: Properties measurement setup.

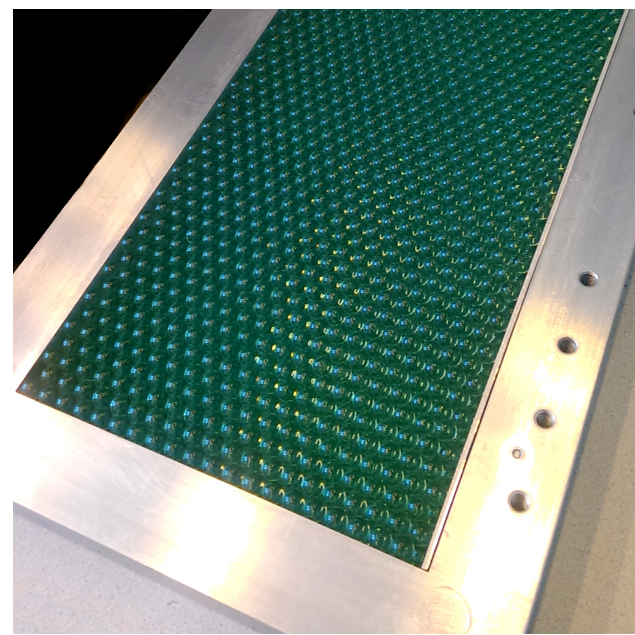

Figure 9: Detail of the box with marbles.

in the box according to a hcp close sphere packing. The glass marbles have a diameter of $12 \mathrm{~mm}$ with tolerance of $0.5 \mathrm{~mm}$. A photo of the box with marbles is shown in Figure 9.

The setup has been placed inside an anechoic room at the University of Twente. A photo of the setup is shown in Figure 10. The anechoic room has the dimensions of $6 \mathrm{~m} \times 6 \mathrm{~m}$ and a height of $3.9 \mathrm{~m}$. The walls are such that all sounds above $200 \mathrm{~Hz}$ are absorbed. The setup and the speaker have been positioned along the diagonal of the anechoic room.

The box with marbles was placed inside a sound

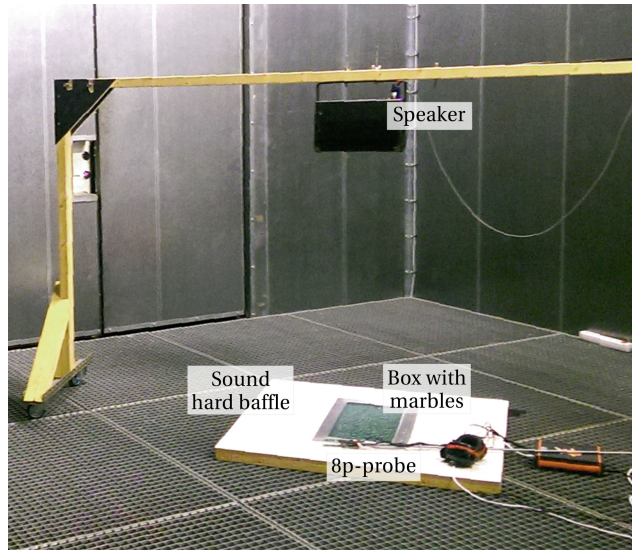

Figure 10: Photo of the setup in the anechoic room at University of Twente.

hard baffle on the floor of this anechoic room. The speaker, a Tannoy VXP 6 with two concentric drivers and built-in amplifier, served as a sound source. The speaker has been mounted to a wooden frame, such that the center of the speaker was $1.3 \mathrm{~m}$ above the surface of the box with marbles. To validate the sound absorption coefficient for normal incidence, the speaker was placed above the geometric center of the box with marbles. For the validation for oblique incident sound waves, the speaker was moved along the diagonal of the anechoic room, such that the position of the center of the speaker was accordingly to the predefined angles of incidence, as listed in Table 2. The height of the speaker was not adjusted during these measurements.

The acoustic pressures were recorded using a small three-dimensional microphone array, containing 8 digital and omnidirectional MEMS-microphones (ADMP441, Analog Devices) and is therefore called the $8 \mathrm{p}$-probe. The microphones are spaced in a cubic array, as shown in Figure 12. For more information about the design, characteristics and implementation of this 8p-probe, the reader is referred to [12]. Furthermore, in [12, 13] the measurement technique is validated for several sound hard and sound absorbing materials for normal and oblique incidence. The same prototype of the $8 \mathrm{p}$-probe is used for the 
measurements here. Note that for the method described in Section 2.3.2, only 2 microphones are required to determine the sound absorption coefficient for oblique incidence. Additional microphones will give more accurate results, since a least squares method is used, as is illustrated in Figure 11.
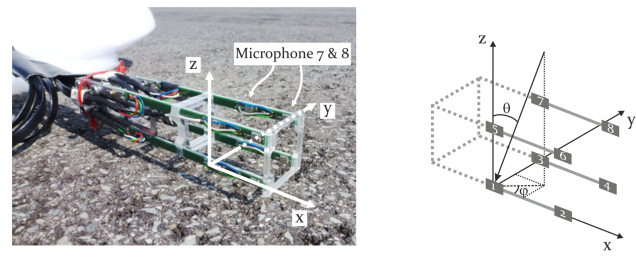

Figure 12: Photo and schematic view of the 8p-probe.

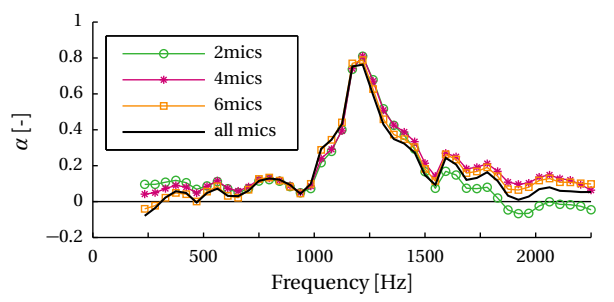

\begin{tabular}{cc}
\hline $\begin{array}{c}\text { Horizontal distance }[\mathrm{m}]: \\
\text { speaker to center setup }\end{array}$ & $\begin{array}{c}\text { Angle of } \\
\text { incidence }[\mathrm{deg}]\end{array}$ \\
\hline 0 & 0 \\
0.42 & 20 \\
0.66 & 30 \\
0.97 & 40 \\
1.99 & 60 \\
\hline
\end{tabular}

Figure 11: Area averaged sound absorption coefficient using different number of microphones for post processing for $\theta=0^{\circ}$.

The 8p-probe has been calibrated using the procedure is described in Appendix B. This resulted in calibration curves, describing the amplitude and phase errors of all microphones compared to a reference microphone. These calibration curves are used whilst processing the acoustic pressure signals.

The acquisition of the pressure signals is performed with a 40-channel digital system and a PC (using a customised LabVIEW program). The signals are analysed in data blocks of 1024 points, with $50 \%$ overlap and using a Hann window. The same PC and LabVIEW program was used to send a white noise sound signal to the speaker, containing frequencies in the range from $200 \mathrm{~Hz}$ to $4000 \mathrm{~Hz}$, with a sample frequency of $48 \mathrm{kHz}$. The calibration procedure showed that the microphone signals up to $f=2250 \mathrm{~Hz}$ are reliable.

The developer of the $8 \mathrm{p}$-probe validated this measurement technique using a linear $x$-y guiding system to measure a large set of grid points, equally divided along the surface and the edges of a (locally behaving) material $[12,13]$. However, this is a very time consuming method and is difficult to apply outside of labora-

Table 2: Angle of incidence for speaker placed at 1.3 $\mathrm{m}$ above the setup with marbles.

tory circumstances. Therefore, a time-effective method which can also be carried out in-situ, is used here. This method is to perform a manual scanning motion with the 8p-probe parallel to the measurement surface. For better accuracy, the results of two different scanning motions of the projected surface are combined; (1) a scan along the longest direction of the box and (2) a scan perpendicular to this direction, along the shortest direction of the box. This is illustrated in Figure 13. The orientation of the 8p-probe and the total measurement time (being $T \approx 28 \mathrm{~s}$ ) was identical for both scanning measurements. The resulting pressure signals have been analysed using the upward z-direction as direction of the surface normal.

As shown in Figure 7 and 8, the circumferential surfaces of the measurement surface have to be included in the measurements as well, such that an enclosed box around the measurement surface is scanned. This is done by separate scans along these circumferential surfaces, as illustrated in Figure 14. The outward surface normal of each surface, used to analyse the pressure signals, are also indicated in this figure.

The resulting area averaged sound absorption coefficient is the energetic average of the scans 
of the projected and circumferential surfaces, according to the theory in Section 2.3.2. The angle of incidence is estimated based on the position of the speaker and the geometrical center of the box, and corrected for the orientation of the 8p-probe itself using the outward surface normal of the projected surface and all circumferential surfaces.
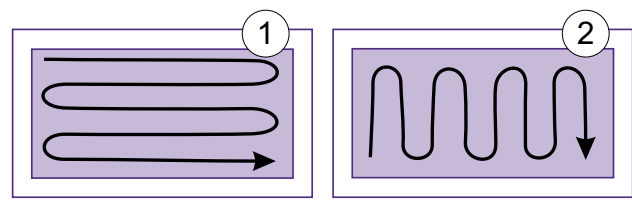

Figure 13: Schematic representation of the path for two different scanning techniques of the projected surface.

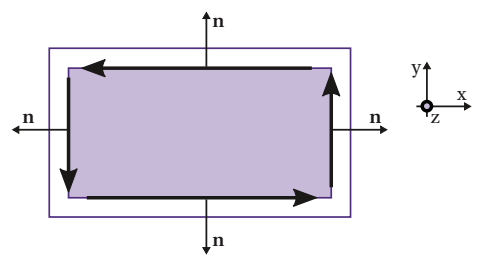

Figure 14: Schematic representation of the path during the scans of the circumferential surfaces, for each edge the outward surface normal $\mathbf{n}$ is indicated.

\subsection{Measurement results}

Measurements are carried out for the cases listed in Table 2. Normal incidence is considered as a special case of oblique incidence. The predicted sound absorption coefficients are compared with the measured absorption coefficients for each angle of incidence, as shown in Figure 15.

\subsection{Measurement uncertainties}

Different scanning techniques are used to obtain more accurate results. Also, additional measurements for $\theta=0^{\circ}$ are performed after the complete setup has been disassembled and reassembled, to estimate the accuracy and measurement repeatability. The overall
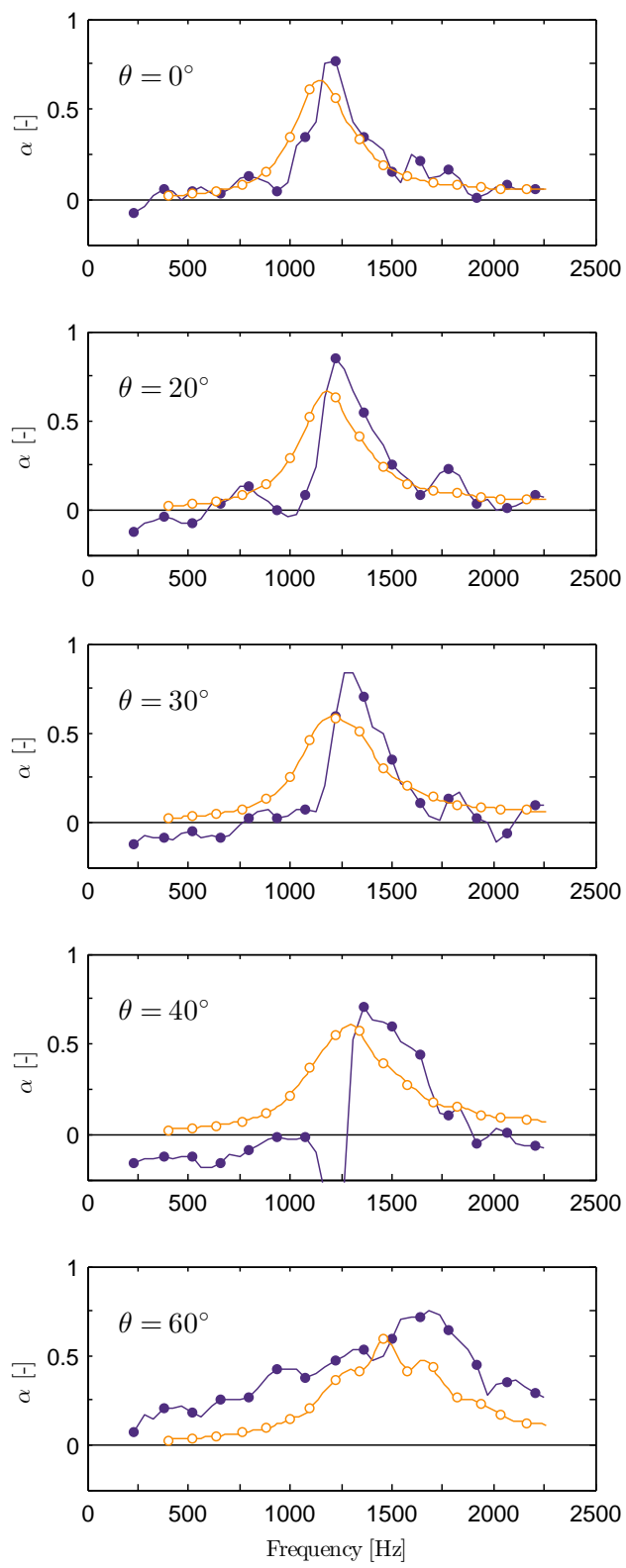

Figure 15: Measured (filled markers) and predicted (open markers) sound absorption coefficient for oblique incidence.

standard deviation, within the frequency range shown, of the sound absorption coefficient for both measurements is about 0.05 , with a max- 
imum value of 0.21 (at $f=1640 \mathrm{~Hz}$ ). Note that only a limited number of measurements is performed and no statistics or measurement uncertainty can be derived.

The variations in the measurement results can be explained by the measurement method and setup. Some causes of the measurement uncertainties are:

- the used (manual) scanning technique, this is elaborated in more detail below;

- alignment of the microphones on the (prototype) 8p-probe;

- scattering on the mounting system of the speaker and scattering on the 8p-probe itself;

- quality of the microphones and possible errors in the calibration method of the pressure signals. Especially the phase error becomes important for low sound absorption and for higher frequencies. Therefore, no measurement data above $f=2250 \mathrm{~Hz}$ are shown.

\subsubsection{Scanning technique}

A manual scanning technique has been used to measure the sound pressure above and around the box with marbles. This is the most timeefficient technique, but not the most accurate technique. It is possible that:

- the scan does not include the complete enclosed surface;

- the orientation of the probe changes (slightly) during the scan;

- the probe vibrates or comes into contact with the measurement surface during the scan;

- the person who performs the scan causes scattering, since he or she is inside the anechoic room during the measurements.

For more repeatable results and also to ensure that an enclosed box around the measurement surface is measured, it is recommended to use a linear $\mathrm{x}$-y guiding system to measure a large enough set of grid points, equally divided along the projected surface and circumferential surfaces. Note that this guiding system is also a possible source of uncertainties, like vibrations, noise of the actuators and cooling system and scattering of the sound waves on the guiding system itself. Furthermore, using a linear guiding system will be time-consuming and not be feasible for in-situ measurements.

\subsubsection{Sound emission}

The results in Figure 15 show that when the sound absorption coefficient is low, the measured coefficient can drop below zero. This indicates sound emission which is defined as:

$$
\alpha_{\text {emission }}=\frac{W_{\mathrm{ac}}}{W_{\mathrm{em}}}=\frac{\int \mathbf{I}_{\mathrm{ac}} \cdot \mathbf{n} d S}{\int I_{\mathrm{em}} d S}
$$

where the intensity of the emitted waves is $I_{\mathrm{em}}=$ $I_{\text {refl }}$. The sound emission coefficient is defined as the ratio between the active sound power and the emitted sound power and is typically between 0 and -1 . A sound emission coefficient of $\alpha_{\text {emission }}=0$ indicates total reflection without sound emission and $\alpha_{\text {emission }}=-1$ indicates that the entire (measured) sound field is emitted by the (measured) object. Sound emission for this measurement setup should not be possible and is expected to be the result of the measurement method or techniques.

The measurement results for an angle of incidence of $\theta=0^{\circ}, \theta=20^{\circ}, \theta=30^{\circ}$ and $\theta=60^{\circ}$ show only negative values for the sound absorption coefficient close to zero, which is the region where the influence of the phase error of the microphones is the largest. Because for surfaces with a low sound absorption, it is more difficult to measure the sound pressure and the phase difference of the signals becomes in the same range as the phase error of the microphones.

The measurement results for an angle of incidence of $\theta=40^{\circ}$ shows larger negative value of the absorption coefficient around $f=1250 \mathrm{~Hz}$. It is assumed this is caused by the manual scanning technique; it could be that the projected and circumferential surfaces are not measured 
properly causing energy leaking from the baffle into the measurement area.

\subsection{Discussion of results}

Figure 15 shows similar trends for the measured and predicted results. The measured and predicted results all show a distinctive peak in the sound absorption coefficient, for which the measured frequency and amplitude corresponds fairly well with the predicted results. However, the predictions of the amplitude of the absorption peak underestimate the measured sound absorption. This is probably caused by a difference in the porosity between the simulation and measurement setup, introduced by the small overlap of the spheres in the numerical model.

Furthermore, some of the measurement results show a (small) second peak in the absorption coefficient around $1800 \mathrm{~Hz}$, this peak is not visible in the predicted sound absorption coefficient. It is possible that this peak is introduced by the measurement method or uncertainties, especially since the correlation between the microphones is low around this frequency. However, this difference can also be introduced by the model implementation, since the numerical model is not an exact representation of the measurement setup. For instance, only a slice of the complete box is used for the numerical model.

Figure 16 shows the measured sound absorption coefficient for the projected and circumferential surfaces as well as the total area averaged sound absorption coefficient for $\theta=0^{\circ}$. The absorption coefficient of the surface parallel to the measurement surface, the projected surface, shows sound absorption. The absorption coefficient of the circumferential surfaces shows mostly sound emission, which indicates that energy is flowing from the measurement surface towards the baffle around the box, as is indicated by the intensity vectors in Figure 8 .

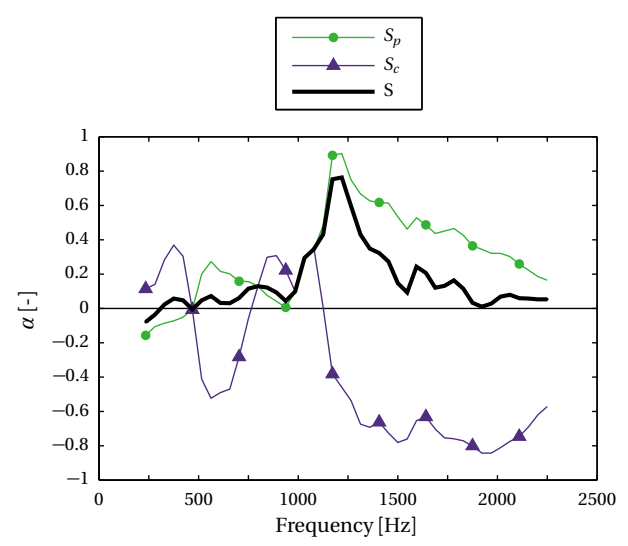

Figure 16: Area averaged sound absorption coefficient from measurements for the projected and circumferential surfaces, $S_{p}$ and $S_{c}$ respectively, for $\theta=$ $0^{\circ}$. The total area averaged sound absorption coefficient is also included (solid black line indicated by $S$ ).

\subsubsection{Tube resonator}

The distinctive peaks around $f=1175 \mathrm{~Hz}$ for $\theta=0^{\circ}$ can be explained when the pores in the granular structure are compared to a tube resonator. Assuming there is a dominant pore between the marbles, the behavior of this pore can be compared to a quarter wavelength tube resonator. The first resonance frequency $f_{1}$ of a quarter wavelength tube with one closed end and one open end and length $L_{\text {tube }}$ is:

$$
f_{1}=\frac{c_{\mathrm{II}}}{4 L_{\text {tube }}}
$$

The analogy with a simple tube resonator gives some insights in the sound absorption behavior of the granular structure, but this method is not accurately enough to predict the resonance frequencies. The developed model, however, predicts the frequency of the absorption peak fairly well. Especially considering that the model is not an exact representation of the measurement setup and the measurement procedure introduced many uncertainties. 


\subsubsection{Behavior for oblique incidence}

The results of the simulations are shown together in one figure, Figure 17, such that the trends in relation to the angle of incidence can be seen. In general, for a larger angle of incidence the frequency of the absorption peak increases. This can be explained when decomposing the wave number $k$ in:

$$
\begin{aligned}
& k_{x}=k \sin \theta_{j} \cos \varphi_{j} \\
& k_{y}=k \sin \theta_{j} \sin \varphi_{j} \\
& k_{z}=k \cos \theta_{j}
\end{aligned}
$$

with $k^{2}=k_{x}^{2}+k_{y}^{2}+k_{z}^{2}$. For $\theta=0^{\circ}$ and $\varphi=$ $0^{\circ}$, the wave number in $\mathrm{z}$-direction corresponds with the first resonance frequency of the quarter wavelength tube: $k_{1}=k_{z}$. As is illustrated in Figure 18, the corresponding resonance wave number in z-direction does not depend on the angle of incidence. Therefore, when the angle of incidence increases, the wave number $k_{1}$ and thus the resonance frequency $f_{1}$ will increase as well.

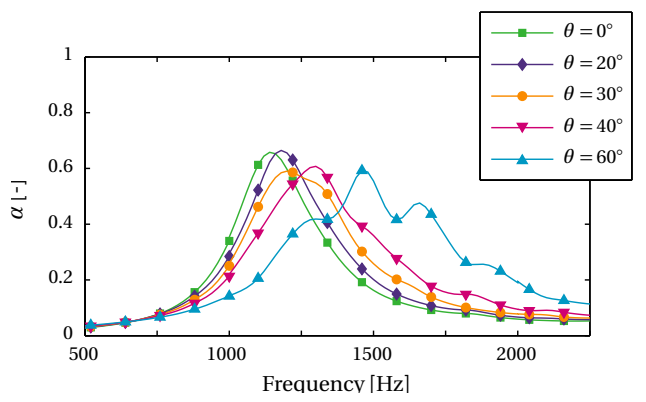

Figure 17: Predicted sound absorption coefficient for oblique incidence.

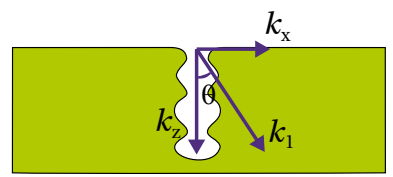

Figure 18: Illustration of resonance frequency for oblique incidence.

Figure 17 also shows that the amplitude of the absorption peak slightly decreases for an increasing angle of incidence. Also, for normal incidence the amplitude of the peak is slightly lower than the one for $\theta=20^{\circ}$, as can be seen in both the measured and predicted results. Furthermore, it can be seen that the width of the absorption peak increases for an increasing angle of incidence. The relation between the angle of incidence and the amplitude of the absorption peak, including the underlying behavior, should be researched further. Some insights on the complex behavior for locally reacting surfaces are given in [1], where is stated that for interconnected pores inside a granular structure, interference between waves inside the pores is possible, because these waves can be out of phase.

\subsubsection{Resonance in longitudinal direction}

The results for $\theta=60^{\circ}$ show multiple peaks in the sound absorption coefficient, about $200 \mathrm{~Hz}$ apart. This frequency corresponds fairly well with the resonance frequencies of the sound waves in the horizontal (longitudinal) direction of the box holding the marbles. In Figure 19, the sound absorption coefficient is compared to a model with the same (viscous) properties for medium II, but without the granular structure. The dimensions and boundary conditions are not changed. In these results, the same periodic behavior is visible. However, for the model without the granular structure, the peak in the sound absorption around $1500 \mathrm{~Hz}$ is not present. This periodic behavior is also visible in the results with a smaller angle of incidence, but is less pronounced here.

For the models with a larger angle of incidence, the effects of scattering on the outer boundaries of the air domain can become a problem. The distance from these outer boundaries to the box with the granular structure is limited in the model and can introduce some scattering. The length of this distance is investigated in a simplified model and it is found that a larger distance will give less scattering. It was not possible to implement a distance much larger than one time the largest wavelength, since the number of elements in the mesh became too large. 


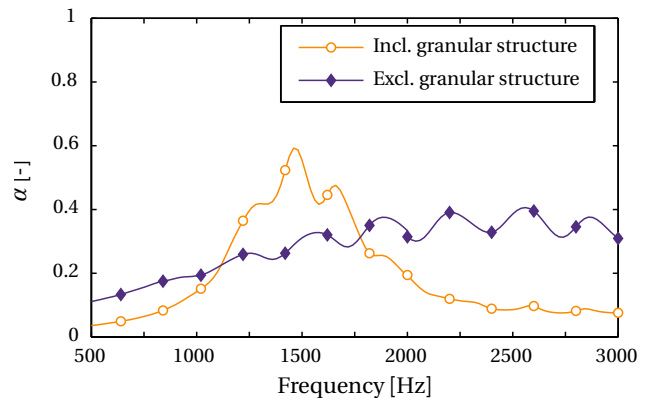

Figure 19: Predicted sound absorption coefficient for model with and without granular structure, for $\theta=$ $60^{\circ}$.

\section{Conclusions}

In this paper, a three-dimensional modeling approach to predict the sound absorption coefficient for porous (granular) materials for oblique incident sound waves is presented. In the future, this modeling approach can be used to predict the sound absorption coefficient for (simplified) porous asphalt concrete in the design phase. Characteristic about this modeling approach is that both the (local) scattering of the sound waves on the stones inside the porous concrete as well as the viscothermal effects inside the air-filled pores are included, while also including the dependence of the sound absorption coefficient on the angle of incidence. One of the important advantages of the developed modeling approach is the relatively low computational time, since (a) no boundary layers are required in the viscous air layer (medium II), and (b) the model dimensions of the surrounding air (medium I) can be kept small, because only a (local) scattering problem is solved.

In this paper, a quick, in-situ method to measure the absorption coefficient for oblique incidence is used to validate this modeling approach. The validation is focused on a structure with stacked marbles. The method to measure the sound absorption for oblique incidence is new and a prototype of the equipment is used, the method and equipment are described by [12] and should be improved for more accurate results. However, the results shown in this paper, show a reasonably good correlation between the measurement results and the predicted results. The trend in the sound absorption coefficient corresponds fairly well for both measurement and simulation. Furthermore, the distinctive peak in the sound absorption coefficient visible in both measurement and simulation results have similar frequency and magnitude for almost all cases. Also, the angle dependency of the sound absorption coefficient seen in the measurement results, is predicted using the developed modeling approach.

\section{Acknowledgement}

This project is carried out in the framework of the innovation program 'GO Gebundelde Innovatiekracht', funded by the 'European Regional Development Fund', 'Regio Twente' and 'Provincie Overijssel'. The project partners are Apollo Tyres Global R\&D, University of Twente (Tire-Road Consortium), Reef Infra, STEMMER IMAGING and the Provincie Gelderland, and their support is gratefully acknowledged.

\section{Appendix A: Low reduced frequency model}

Below, a summary of the most important relations of the low reduced frequency (LRF) model are listed. All relations shown here are based on the sound field in a tube with circular crosssection. A more detailed discussion of the LRF model can be found in $[4,10,17,18]$.

The sound pressure $p(\omega, z)$ inside this tube is given by:

$$
\begin{aligned}
p(\omega, \mathbf{r}, \theta, \varphi)= & A e^{\Gamma(\omega) k_{j}(\omega) \xi_{j}(\mathbf{r}, \theta, \varphi)} \\
& +B e^{-\Gamma(\omega) k_{j}(\omega) \xi_{j}(\mathbf{r}, \theta, \varphi)}
\end{aligned}
$$

where $\Gamma(\omega)$ is the viscothermal wave propagation coefficient. The wave propagation number in this tube $k_{j}$ is related to the wave propagation number in standard air conditions by:

$$
k_{j}(\omega)=-i k(\omega) \Gamma(\omega)
$$


The solution for the particle velocity in axial direction, averaged over the cross-section of the tube, yields:

$$
\begin{aligned}
\bar{\nu}(\omega, \mathbf{r}, \theta, \varphi)= & \frac{G(\omega)}{\rho_{0} c_{0}}\left[A \cos \psi e^{\Gamma(\omega) k(\omega) \xi(\mathbf{r}, \theta, \varphi)}\right. \\
& \left.-B \cos \psi e^{-\Gamma(\omega) k(\omega) \xi(\mathbf{r}, \theta, \varphi)}\right]
\end{aligned}
$$

where $A$ and $B$ are the complex amplitudes of the incident and reflected waves, $\rho_{0}$ and $c_{0}$ the density and speed of sound assuming standard air conditions and the coefficients $\Gamma$ and $G$ depending on both the angular frequency $\omega$ as well as the geometry of the cross-section. For a tube with circular cross-section these coefficients yield:

$$
\begin{aligned}
\Gamma(\omega) & =\sqrt{\frac{J_{0}(i \sqrt{i} s(\omega))}{J_{2}(i \sqrt{i} s(\omega))} \frac{\gamma}{n}} \\
G(\omega) & =-\frac{i}{\Gamma(\omega)} \frac{\gamma}{n}
\end{aligned}
$$

with the shear wave number $s$ and coefficient $n$ :

$$
\begin{aligned}
& s(\omega)=r_{\mathrm{t}} \sqrt{\frac{\rho_{0} \omega}{\mu}} \\
& n(\omega)=\left[1+\frac{\gamma-1}{\gamma} \frac{J_{2}\left(i \sqrt{i N_{\mathrm{pr}}} s(\omega)\right)}{J_{0}\left(i \sqrt{i N_{\mathrm{pr}}} s(\omega)\right)}\right]^{-1}
\end{aligned}
$$

where $J_{0}$ and $J_{2}$ are Bessels functions of the first kind of order 0 and 2, $\mu$ is the dynamic viscosity, $\gamma=C_{p} / C_{v}$ is the ratio of specific heats, $N_{\mathrm{pr}}=$ $\mu C_{p} / \kappa$ is the Prandtl number.

\section{Appendix B: Calibration procedure 8p-probe}

The cubic microphone array, the $8 p$-probe, is calibrated using the free-field technique, following the procedure described by [11]. Again, the anechoic room at the University of Twente has been used. The setup consisted of the $8 p$ probe, fixed in upright position at a height of $H \approx 1 \mathrm{~m}$, and the speaker, mounted at a height of $H \approx 0.8 \mathrm{~m}$ and at a distance of $4.8 \mathrm{~m}$ to the probe.

Four series of measurement were carried out and for each measurement series the probe was aligned such that 2 planes containing 4 microphones each, were positioned perpendicular to the direction of sound. All four positions, when maintaining the upright position - thus rotating around the $\mathrm{x}$-axis, as indicated in Figure 12 - were measured. Each series contained 3 measurements. The calibration method is illustrated in Figure 20 and 21.

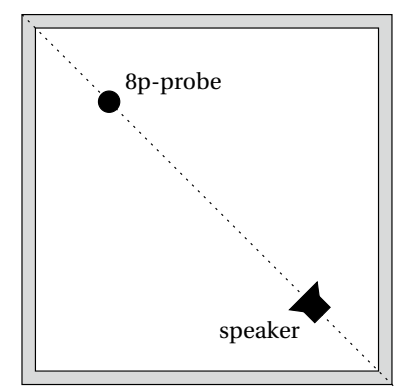

Figure 20: Illustration of measurement setup during calibration of 8p-probe.
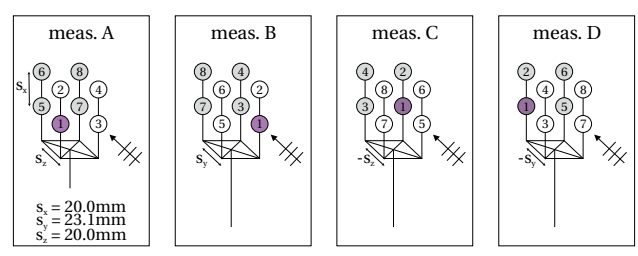

Figure 21: Four orientations of the 8p-probe during calibration measurements.

The sound pressure level and the phase difference for all microphones at both parallel planes can be derived when assuming local plane waves between the two planes. For each microphone the amplitude error and phase error has been derived, by taking the transfer functions from $i$ th microphone to the reference microphone, here microphone 1 , giving 8 complex transfer functions in total. The transfer functions have been averaged within a series of three measurements before taking the spatial average for the 4 different positions of the probe.

Through each obtained transfer functions, a curve has been fitted. Each transfer function $H$ is of the form: $H=\left|A_{\mathrm{TF}}\right| e^{(i \Psi)}$, where $\left|A_{\mathrm{TF}}\right|$ is the magnitude of the complex amplitude and $\Psi$ the 
phase error. The curves fitted through the transfer functions are a combination of a polynomial function of the first order for both the amplitude and the phase:

$$
H_{\text {cal }}(\omega)=Y(\omega) e^{i \Psi(\omega)}
$$

where $Y(\omega)$ is the amplitude, fitted through the curve:

$$
Y(\omega)=a_{1} \omega+a_{2}
$$

and $\Psi(\omega)$ the phase error, given by:

$$
\Psi(\omega)=a_{3} \omega+a_{4}
$$

The behavior of both the amplitude error and the phase error for the microphones at the end of the 8p-probe, microphones 2, 4, 6 and 8 in Figure 12, show an oscillation around the fitted curve for frequencies above $f \approx 2000 \mathrm{~Hz}$. It is assumed that this oscillation is introduced by scattering of the sound waves on the probe itself. This effect should be investigated further before this can be included in the calibration of the 8p-probe. For now, the linear calibration curves are used and the pressure signals up to $f=2250 \mathrm{~Hz}$ are used for the validation, since for these frequencies the phase errors are small.

\section{References}

[1] J. Allard and N. Atalla. Propagation of Sound in Porous Media: Modelling Sound Absorbing Materials $2 e$. John Wiley \& Sons, 2009.

[2] K. Attenborough, I. Bashir, and S. Taherzadeh. Outdoor ground impedance models. J. Acoust. Soc. Am., 129:2806-2819, 2011.

[3] K. Attenborough, K. M. Li, and K. Horoshenkov. Predicting outdoor sound. Taylor \& Francis, 2007.

[4] W. M. Beltman. Viscothermal wave propagation including acousto-elastic interaction. $\mathrm{PhD}$ thesis, University of Twente, 1998.
[5] M. C. Berengier, M. R. Stinson, G. A. Daigle, and J. F. Hamet. Porous road pavements: Acoustical characterization and propagation effects. J. Acoust. Soc. Am., 101(1):155162, 1997.

[6] M. Bezemer-Krijnen, Y. H. Wijnant, and A. de Boer. Predicting sound absorption of stacked spheres: combining an analytical and numerical approach. Acta Acustica united with Acustica, 102:971-983, aug 2016.

[7] M. A. Biot. Theory of propagation of elastic waves in a fluid-saturated porous solid. i. low-frequency range. Acoustical Society of America, 28(2):168-178, march 1956.

[8] M.A. Biot. Theory of propagation of elastic waves in a fluid-saturated porous solid. ii. higher frequency range. Acoustical Society of America, 28(2):179-191, march 1956.

[9] D. T. Blackstock. Fundamentals of physical acoustics. John Wiley \& Sons, 2000.

[10] M. H. C. Hannink. Acoustic resonators for the reduction of sound radiation and transmission. PhD thesis, University of Twente, 2007.

[11] E. R. Kuipers. Measuring sound absorption using local field assumptions. $\mathrm{PhD}$ thesis, University of Twente, 2013.

[12] E. R. Kuipers, Y. H. Wijnant, and A. de Boer. Measuring oblique incidence sound absorption using a local plane wave assumption. Acta Acustica united with Acustica, 100(2):205-214, 2014.

[13] E. R. Kuipers, Y. H. Wijnant, and A. de Boer. Measuring sound absorption: considerations on the measurement of the active acoustic power. Acta Acustica united with Acustica, 100(2):193 - 204, 2014.

[14] F. P. Mechel. Sound fields at periodic absorbers. J. Sound Vib., 136(3):379-412, 1990.

[15] M. R. Stinson. The propagation of plane sound waves in narrow and wide circular tubes, and generalization to uniform tubes of arbitrary cross-sectional shape. 
Acoustical Society of America, 89(2):550558, February 1991.

[16] M. R. Stinson and Y. Champoux. Propagation of sound and the assignment of shape factors in model porous materials having simple pore geometries. J. Acoust. Soc. Am., 91(2):685-695, 1992.

[17] H. Tijdeman. On the propagation of sound waves in cylindrical tubes. J. Sound Vib., 39(1):1-33, 1975.

[18] F. J. M. van der Eerden. Noise reduction with coupled prismatic tubes. $\mathrm{PhD}$ thesis, University of Twente, 2000.

[19] Y. H. Wijnant, E. R. Kuipers, and A. de Boer. Development and application of a new method for the insitu measurement of sound absorption. In Proceeding of ISMA2010 including USD2010, pages 109122, 2010.

[20] C. Zwikker and C. W. Kosten. Sound absorbing materials. Elsevier, 1949. 



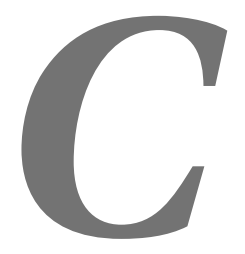

\section{Integral approach to tire/road noise: considering both tire and road design}

This chapter is based on the paper 'Integral approach to tire/road noise: considering both tire and road design', submitted to 'Acta Acustica united with Acustica' (2018). 


\title{
Integral approach to tire/road noise: considering both tire and road design
}

\author{
M. Bezemer-Krijnen ${ }^{1)}$, Y.H. Wijnant ${ }^{1)}$, A. de Boer ${ }^{1)}$ \\ submitted to Acta Acustica united with Acustica (2018) \\ 1) Faculty of Engineering Technology, University of Twente, P.O. Box 217, 7500 AE Enschede, \\ The Netherlands. \\ m.bezemer@utwente.nl
}

\begin{abstract}
Traffic noise is a well known problem, especially for densely populated areas. Many different mechanisms influence the noise level and sound radiation, like the type of the road surface, the design of the tires, and the interaction between the tires and the road. There are various well-known methods to measure tire/road noise and in this paper some of these methods are used to determine the influence of both the road and tire characteristics on the noise radiation, using CPX (close-proximity) measurements, CPB (controlled pass-by) measurements, sound absorption measurements, and road roughness measurements. The road parameters which have been looked into are the porosity of the road, layer thickness, stone sizes, the road roughness, and the sound absorption coefficient, for both normal and oblique incidence. The tire design parameters have been based on the (simplified) tread design. The research and measurements have been performed within the project 'Stil Veilig Wegverkeer' (translated in: 'Silent and Safe Road Traffic'). The main goal of this project is to find the optimal tire/road combination for safe and silent road traffic. In this paper we concentrate on finding a silent tire/road combination and to map the influence of some of the design properties of both the road and the tires. The measurements have taken place on a special test area in the Netherlands, where eight different type of asphalt surfaces were constructed. Two of these tracks have been constructed based on new modelling techniques to predict the sound absorption and grip. Three tires with different tire profiles were used during these measurements. The measurements show that the sound absorption coefficient and the porosity of the road are important design parameters to reduce the sound radiation for higher frequencies. For the lower frequencies, the largest sound reduction is obtained by minimising the excitation mechanisms for tire vibrations, for instance by minimising the road roughness, stone size and tire pattern continuity. An optimal combination of tire and road surface would be a tire with little variation in the pattern along the circumference of the tire and an open road surface with a smooth surface.
\end{abstract}




\section{Introduction}

The main source of tire/road noise, for speeds above about $40 \mathrm{~km} / \mathrm{h}$, is caused by the interaction between the tires of a car and the road surface [32]. The variation in contact force between the tire and road while driving introduces vibrations and sound radiation. Traffic noise can be reduced by decreasing the sound excitation mechanisms - for example by changing the width or stiffness of the tire, the design of the tire tread, or the road roughness - or by decreasing the sound radiation - by changing the sound absorption of the road or using noise barriers. Tire/road noise is investigated extensively and there are various (well-known) methods to measure the radiated noise, like the close-proximity method [6] and pass-by methods [1]. Overviews of the different excitation mechanisms and measurement methods are, for example, given by [10, 16, 20, 33]. From literature, it is clear that tire/road noise is a very complex problem and depends on the tire, vehicle and the road surface.

Various numerical models have been created to get more insights in the excitation mechanisms and to predict the sound radiation from a rolling tire. An extensive overview of the available models is given by [26]. In addition, numerous measurements have been performed to find relations between the sound radiation and tire parameters that influence the excitation mechanisms. As stated in [33], it is very difficult to find correlations between the different studies. Mostly because many mechanisms depend on multiple parameters and have sometimes counteracting effects. Parameters that can influence the results are:

- type of tires (width, diameter, tread pattern, stiffness of tread blocks and tire [9, $18,33]$ );

- condition of the tires (age, rubber hardness [7, 36]);

- measurement methods (indoor using a drum, outdoor on different road surfaces and velocities);

- measurement environment (temperature, solar radiation $[7,36])$.

Furthermore, much has been written about the road characteristics that influence the tire/road noise, for example the surface texture and the sound absorption coefficient of the road surfaces $[21,25,27,32]$. The sound absorption coefficient depends on road characteristics such as the size of the aggregates or stones, the variation in the sizes of the stones or aggregates, the porosity or air void content, the connectivity of the air voids and the layer thickness of the road surface [21, 25, 27, 30-32]. In general the following conclusions based on measurements can be found:

- for low frequencies, the road roughness induces tire vibrations, and the noise levels increase for a larger road roughness $[21,32]$;

- for high frequencies, the road roughness can (partly) decrease the noise levels, since the texture of the road surface influences effects such as air pumping and stick-slip of the tread blocks [21, 32];

- (semi-)porous road surfaces can significantly decrease the sound radiation [25, $27,30]$;

- a larger air void content and connectivity of the air voids increase the sound absorption coefficient, which decreases the noise levels [25];

- the layer thickness influences the frequency and amplitude of the first peak in the sound absorption coefficient, a larger layer thickness corresponds with a lower frequency and a lower peak $[25,27,30]$.

Also, various phenomenological and (semi-) analytical models exist to predict the sound absorption coefficient of road surfaces based on these parameters, for example $[8,13,15,17$, 19].

The main objective of this research is to find relations between both tire and road characteristics and tire/road noise, contrary to most literature in which the focus is on sound radiation of random vehicles, tire or road parameters. Using this integral approach of the tire/road inter- 
action, silent combinations of tire and road surface are discussed in this paper. Special attention will be given to the influence of oblique incident sound waves, since tire/road noise will radiate predominantly in the direction parallel to the road surface, as is indicated in Figure 1.
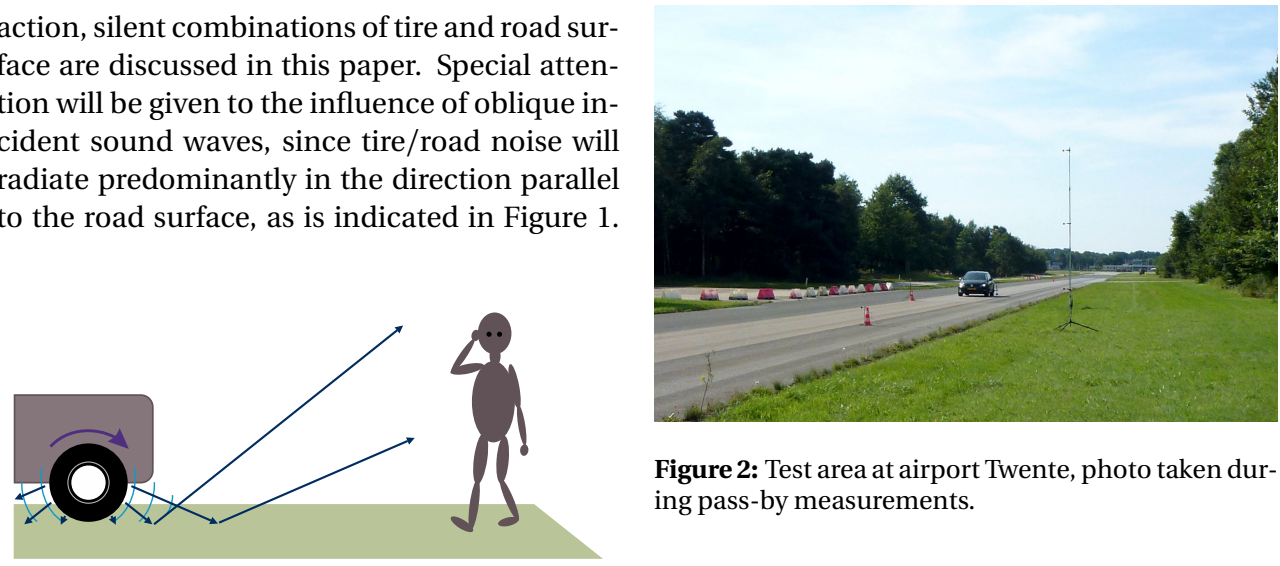

Figure 2: Test area at airport Twente, photo taken during pass-by measurements.

Figure 1: Illustration of sound radiation for traffic noise.

Furthermore, the design and performance of two road surfaces based on predictive models is discussed in more detail in this paper.

\section{Methodology and parameters}

This research is carried out within the project 'Silent and Safe Road Traffic' (in dutch: 'Stil Veilig Wegverkeer', SVW). The project led to an integral approach of the tire/road interaction, both for noise and for wet grip. Within this project, multiple simulation tools and test procedures were developed for the optimisation of a silent and safe tire and road combination. An overview of the different aspects of this project are given in [11] and the different research aspects are discussed in more detail in [11], [28], [12] and [29].

Furthermore, a special test area was developed at Airport Twente, an out-of-service airport in a quiet and rural area in the eastern part of the Netherlands. At this site, 8 tracks with a different type of asphalt surfaces were constructed. The properties of the test tracks are listed in Appendix A. Six tracks were constructed in 2013. In combination with predictive models and design tools, two new road surfaces were developed and constructed in 2015, designed to have

minimal sound radiation and best grip. The design of these two test tracks is discussed in detail in Section 3.

An impression of the test area is shown in Figure 2.

All road surfaces have been tested using various tires. Numerous noise measurements have been performed during this project, such as statistical pass-by measurements, controlled passby measurements, close-proximity measurements using a trailer and a car and sound absorption measurements. In this paper we focus on only a selection of all the parameters that can influence the tire/road noise problem. These parameters and results are analysed to find global trends.

\subsection{Tire parameters}

As this research is mostly focused on the influence of the road design parameters, the tire parameters are kept simple and are based on an estimate of the footprint of each tire. Furthermore, influences of effects as the velocity, rubber stiffness, aging and environmental circumstances have not been included in this study. Therefore, detailed information from the tire manufacturer about the tires is not required, which is an advantage, since this data is often hard to get.

In this study, three tires are used for the noise measurements. These are the standard reference test tire (SRTT Tigerpaw, Uniroyal), the Ul- 

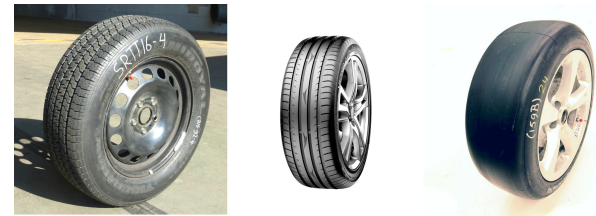

Figure 3: The three tires used for the sound radiation measurements: (left) SRTT Tigerpaw, (center) Ultrac Cento XL and (right) slick tire.
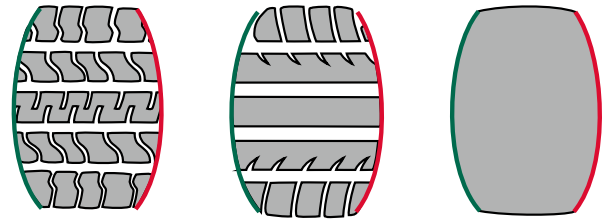

Figure 4: Schematic rendering of footprint area of SRTT Tigerpaw (left), Ultrac Cento (center) and the slick (right), where the dark green line indicates the leading edge and the light red line the trailing edge.

trac Cento XL (Vredestein) and a slick tire. The tires are shown in Figure 3. The schematically rendered footprint areas of these tires are shown in Figure 4.

Two tire parameters are determined to find the influence of the tire and the tire/road combination on the sound radiation. The parameters are listed in Tables 1 and 2 and discussed below.

\subsubsection{Pattern continuity}

One of the excitation mechanisms during tire/road contact are the vibrations due to the variation in contact, caused by the contact forces on the tire and tread blocks during rolling. To describe this variation in contact force along the circumference of the tire, a pattern continuity factor $C_{\text {pattern }}$ is defined to describe the variation in contact area during rolling. This parameter is based on the variation of the ratio between the width of the tread blocks and the total width of the tire footprint $W_{\text {total }}$ along the circumference of the

\begin{tabular}{ccccc}
\hline & & SRTT & Ultrac Cento & Slick \\
\hline$C_{\text {pattern }}$ & {$[-]$} & 0.75 & 0.83 & 1 \\
\hline
\end{tabular}

Table 1: Pattern continuity parameter for the three tested tires.

tire, yielding:

$$
\begin{array}{r}
C_{\text {pattern }}=1-\left[\max \left(\frac{\sum W_{\text {tread }, i}(x)}{W_{\text {total }}}\right)-\right. \\
\left.\min \left(\frac{\sum W_{\text {tread }, i}(x)}{W_{\text {total }}}\right)\right]
\end{array}
$$

The tread pattern is considered in the direction perpendicular to the circumference, as is illustrated in Figure 19. Here is indicated how the the width of the tire blocks in apparent contact $W_{\text {tread }}$ is determined in the center of the footprint.

The pattern continuity factor for the three tires is listed in Table 1. For the slick, the pattern continuity is equal to one, since there is no variation in tread design. The continuity factor is lower for the SRTT Tigerpaw than for the Ultrac Cento, indicating the variation in tread pattern of this tire during rolling is larger.

This pattern continuity factor is a simplified version of the tread pattern height factors discussed by Bekke [10, page 72-81]. However, for the parameter discussed by Bekke, a detailed knowledge of the tire has to be known, while the pattern continuity $C_{\text {pattern }}$, as defined in Equation 1, can be derived from an estimate of the tread pattern based on the footprint area.

Another noise generation mechanism caused by the shape or contour of the tread pattern is discussed by Sandberg and Ejsmont [33, chapter 10]. When the contour of the tread coincides with the contour of the leading or trailing edge of the footprint of the tire, the impact (or release at the trailing edge) will be abrupt and cause vibrations and thus noise. This effect is not included in the pattern continuity, but from Figure 4 is clear that the contours of the tread blocks for both the SRTT Tigerpaw and the Ultrac Cento do not follow the contour of the footprint. 


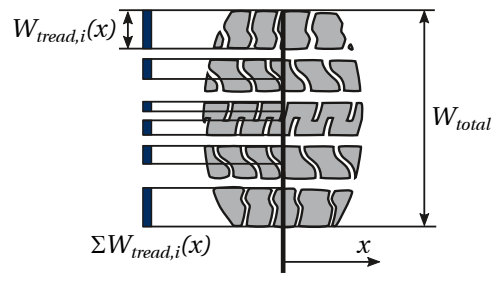

Figure 5: Illustration to demonstrate how the width of the tread blocks in contact at the line through the center of the footprint are determined.

\subsubsection{True contact ratio}

The second parameter does not only depend on the tire, but also on the road surface. This is the true contact ratio $R_{\mathrm{tc}}$ and is the ratio between the real contact area of the tire with the road surface and the total area of the footprint:

$$
R_{\mathrm{tc}}=1-\frac{\left(1-\Omega_{\mathrm{road}}\right) S_{\text {tread }}}{S_{\text {total }}}
$$

where $S_{\text {tread }}$ is the apparent contact area of the tire, $S_{\text {total }}$ the total area of the footprint and $\Omega_{\text {road }}$ the porosity of the road. This is illustrated in Figure 6. Again, a simple method based on the footprint area is used to determine this true contact area. A more advanced method to determine this parameter would be to use footprint images of the tires on every road surface, but this data is not available.
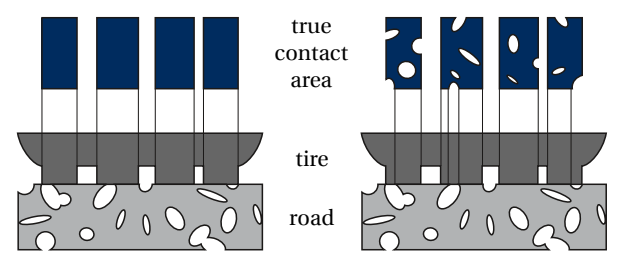

Figure 6: Schematic representation of the apparent contact (left) and the true contact (right).

\subsection{Road parameters}

Based on the literature, the following road parameters seem to have the largest influence on the tire/road noise and are therefore further investigated in this paper:
- the porosity or air void content, indicated by $\Omega_{\text {road }}$;

- the layer thickness, indicated by $H_{\text {road; }}$;

- the aggregate or stone size and variation, indicated by the minimum stone size $D_{s, \min }$ and the maximum stone size $D_{s, \max }$;

- the road roughness, indicated by the mean profile depth (MPD) and the variation in texture height $z_{\text {rms }}$;

- the sound absorption coefficient, indicated by $\alpha$.

The first three parameters are based on the information from the road construction company and are listed in Table 3. The road roughness parameters and the sound absorption coefficient are measured after construction of the test tracks and are discussed in more detail in Section 4 . Note that the first three parameters also influence the sound absorption coefficient. The influence of all parameters on the sound radiation is discussed in Section 5.

\section{Design of prototype road sur- faces}

Within the SVW project, a hybrid analytical/numerical model to predict the sound absorption of porous (granular) materials has been developed $[13,15]$. Using this model, the influence of road design parameters, like the porosity, layer thickness and stone size, on the sound absorption coefficient can be found. Furthermore, this modeling approach also provides the sound absorption coefficient for oblique incidence. Therefore, the sound absorption coefficient can be optimised for large angles of incidence, since the noise radiation caused by traffic noise is mostly a problem for oblique incidence as is illustrated in Figure 1.

The results of this hybrid model and of the measurements performed at the test area in the period between 2013 and 2014 have been used to design two prototype tracks for the test area. These tracks were not only optimised for a low sound radiation, but also for (wet) grip, as discussed by [11, 28]. 


\begin{tabular}{lcccccccc}
\hline & Track 1 & Track 2 & Track 3 & Track 4 & Track 5 & Track 6 & Track 7 & Track 8 \\
\hline SRTT & 0.45 & 0.47 & 0.50 & 0.40 & 0.43 & 0.40 & 0.53 & 0.53 \\
Cento & 0.44 & 0.46 & 0.50 & 0.39 & 0.42 & 0.39 & 0.53 & 0.53 \\
Slick & 0.12 & 0.15 & 0.20 & 0.03 & 0.08 & 0.03 & 0.25 & 0.25 \\
\hline
\end{tabular}

Table 2: True contact ratio, $R_{\mathrm{tc}}=1-\frac{\left(1-\Omega_{\text {road }}\right) S_{\text {tread }}}{S_{\text {total }}}$.

\begin{tabular}{lcccccccc}
\hline & Track 1 & Track 2 & Track 3 & Track 4 & Track 5 & Track 6 & Track 7 & Track 8 \\
\hline$\Omega_{\text {road }}[\%]$ & 12 & 15 & 20 & 3 & 8 & 3 & 25 & 25 \\
\hline$H_{\text {road }}[\mathrm{mm}]$ & 25 & 30 & 57 & 40 & 35 & 35 & 75 & 40 \\
\hline$D_{s, \min }[\mathrm{mm}]$ & 2 & 2 & 4 & 2 & 4 & 2 & 2 & 2 \\
$D_{s, \max }[\mathrm{mm}]$ & 5 & 5 & 8 & 16 & 8 & 8 & 4 & 6 \\
\hline
\end{tabular}

Table 3: Road properties of the test tracks, where $\Omega_{\text {road }}$ is the porosity or air void content, $H_{\text {road }}$ is the layer thickness, $D_{s, \min }$ is the minimum stone diameter in the mixture and $D_{s, \text { max }}$ the maximum stone diameter.

\subsection{Hybrid model as design tool}

The hybrid analytical/numerical model can be used to find the sound absorption coefficient and the sound field in and above a sound hard porous structure. For this paper, the the porous asphalt concrete is modeled as a regular packing of sound hard, equally sized spheres. Since this is not a realistic representation of the porous asphalt concrete, only trends in the relation between the sound absorption coefficient and the road parameters can be predicted and not absolute values. However, the authors are confident that this modelling approach will give insights in the influence of design parameters such as the stone size, porosity and layer thickness on the sound absorption coefficient, specifically for oblique incident sound waves.

The frequency dependent sound absorption coefficient for a granular structure with equally sized round spheres resembles the sound absorption coefficient of a tube resonator, since both curves have a peak in the sound absorption coefficient at a certain (resonance) frequency. When the considered frequency range is large enough, a second and possibly third peak (the higher harmonics) are observed. This behavior is explained in more detail in [13, 15].

Figure 7 shows the predicted sound absorption coefficient for a reference situation with $\Omega_{\text {road }}=$

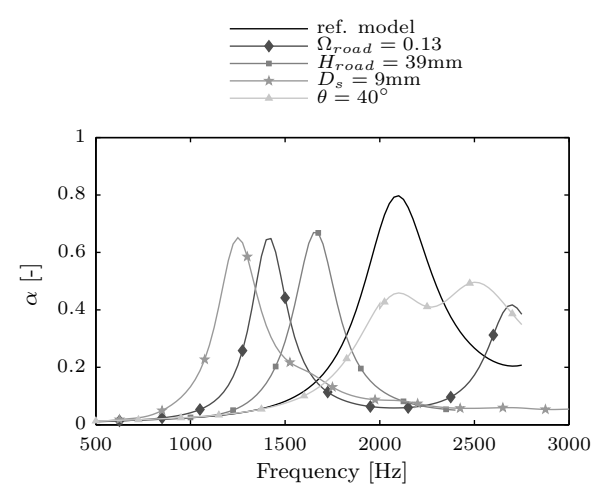

Figure 7: Predicted sound absorption coefficient for a reference model (ref. model) with $\Omega_{\text {road }}=0.19$, $H_{\text {road }}=25 \mathrm{~mm}, D_{s}=6 \mathrm{~mm}$ and $\theta=0^{\circ}$ and the sound absorption coefficient for several variations in the road design parameters.

$19 \%, H_{\text {road }}=25 \mathrm{~mm}$ and $D_{s}=6 \mathrm{~mm}$ for normal incident sound waves. To find the influence of the road parameters, several simulations based on this reference model are performed, while varying only one of the parameters.

Porosity of granular structure: The first parameter that is considered, is the porosity of the granular structure. Figure 7 shows the sound absorption coefficient for $\Omega_{\text {road }}=19 \%$ (ref. model) and $\Omega_{\text {road }}=13 \%$. The trend found 
with the simulations shows that for a larger porosity the peak seen in the sound absorption coefficient becomes wider and shifts to higher frequencies. Also, the amplitude of the sound absorption coefficient is larger for an increasing porosity, both for normal incidence and for oblique incidence.

Layer thickness: The second parameter that is considered is the layer thickness, the variation on the reference model shown in Figure 7 is for $H_{\text {road }}=39 \mathrm{~mm}$. The simulations show that the sound absorption peak shifts to lower frequencies for a larger layer thickness, which is expected when the comparing the behavior to that of tube resonators. Also, the sound absorption coefficients in Figure 7 show a small decrease of the amplitude of the peak for an increasing layer thickness.

Stone size: Another parameter that is considered is the stone size, the results for $D_{s}=9 \mathrm{~mm}$ are shown in Figure 7 . To maintain the same porosity of the structure, the spheres are modelled with a small overlap.

The results of the simulations show that for larger spheres, the amplitude of the peak decreases. Also, the frequency of the absorption peak shifts to lower frequencies for an increasing stone size. One of the possible explanations is that the structure of pores and air pockets between the spheres is less homogeneous for larger sphere sizes, which results in a decrease of the frequency of the peak in the sound absorption coefficient.

Furthermore, for smaller sphere sizes, the peak in the sound absorption coefficient is wider. This could be explained by the viscothermal behavior of the air inside the pores, since for smaller pores, the viscothermal effects and thus the sound absorption will increase.

Angle of incidence: The simulation results for oblique incidence $\left(\theta=40^{\circ}\right.$ in Figure 7) show a second peak in the sound absorption coefficient. This behavior agrees with the behavior for a panel with tube resonators with two different lengths. Furthermore, it is seen in Figure 7 that the peaks have a lower amplitude and have shifted to higher frequencies. Therefore, the sound absorption of the road surface will be lower for oblique incidence than for normal incidence.

Discussion of parameters: In conclusion, the simulations show that:

- in general, the sound absorption coefficient is larger for a higher porosity;

- the width of the peak increases with an increasing porosity and for decreasing stone size;

- the amplitude of the peak depends on porosity, decreases for an increasing layer thickness and angle of incidence;

- the peak shifts to higher frequencies for an increasing porosity, decreasing stone size, decreasing layer thickness and an increasing angle of incidence.

\subsection{Design of prototype tracks}

The model results have been used to optimise the sound absorption coefficient, such that the sound radiation for oblique incidence will be reduced as much as possible. The design criteria given by the model to optimise the sound absorption coefficient, have been combined with additional design criteria to reduce the sound radiation. These are based on conclusions found in the literature and with measurements. Furthermore, the road surfaces have also been optimised for (wet) grip, this is discussed by [11,28]. One of their conclusions was that the wet grip was optimal when the road surface was grinded after construction, to obtain a road surface with microtexture. The process is shown schematically in Figure 9 and is discussed later. Another conclusion was that the surface porosity of the true contact area of the tire/road combination should be small enough such that true contact area between tire and road is maximised and high enough such that drainage of water in the contact area is possible to prevent aqua planing.

Together with the conclusions for noise reduction (both for sound absorption and for sound radiation), this has resulted in the following requirements: 
- a high porosity;

- a large layer thickness;

- a small stone size;

- a smooth road surface or a road surface with negative texture.

Priority was given to the research into (wet) grip, however, both a smooth road surface and a high porosity are also beneficial to minimise sound radiation.

Furthermore, some construction constraints had to be taken into account. A porosity above $25 \%$ is not advisable based on robustness and durability. It was not possible with available machines and techniques to construct a single layered road surface according to these specifications. Therefore, it was chosen to develop one single layered protoype track with the maximum possible layer thickness for the desired combination of porosity and stone size, and a double layered track with the desired layer thickness.

This resulted in a double layered porous asphalt concrete with small stones in the top layer $(2 / 4 \mathrm{~mm})$ and larger stones in the second layer $(8 / 11 \mathrm{~mm})$, track 7 . And in a single layered road surface with stones in the range of $2 / 6 \mathrm{~mm}$, track 8 . The porosity of both tracks is approximately $25 \%$. Both tracks contain a different type of stones, such that the influence of the material on noise and wet grip could be investigated further. The properties of the two prototype tracks, track 7 and track 8 , are summarized in Appendix A.

\section{Measurements}

Various measurements to determine the road characteristics and the noise levels of the test tracks have been performed.

\subsection{Road roughness measurements}

The road roughness is one of the excitation mechanisms of tire/road noise. In general, a

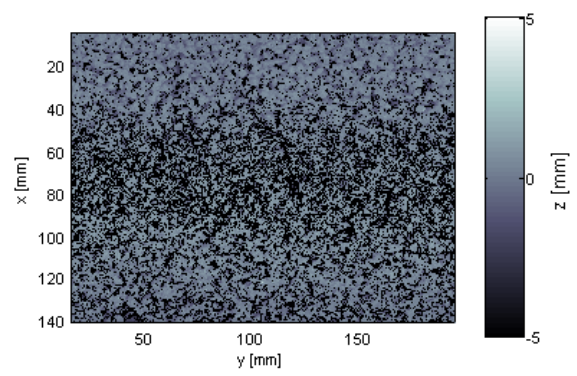

Figure 8: Example of depth image by 3D laser scanner of track 7 .

smoother road will give less excitation and thus less tire/road noise. There are several existing methods to measure the road roughness, most of these methods involve some sort of line scanner attached to a linear guide or a car. Within the project 'Silent and Safe Road Traffic', a 3D laser scanner with two sets of lasers using triangulation has been developed. The 3D scanner acquires a depth image of a surface with texture of $140 \mathrm{mmx} 200 \mathrm{~mm}$, with a resolution of $50 \mu \mathrm{m}$. This scanner is used to scan the surface of all 8 test tracks. For this paper, this depth image is converted into commonly used parameters, such as the mean profile depth (MPD) and the variation in texture height, expressed as the root mean square value of the texture depth $\left(z_{\mathrm{rms}}\right)$. Furthermore, the texture profile level of the tracks is discussed here $[4,35]$. The results are listed in Table 4 and Figures 10 and 11. An example of the depth image of track 7 is shown in Figure 8.

Since the test tracks were also tested for various grip measurements, the bitumen film had to be removed beforehand. The bitumen is removed with a grinding procedure using large rotating diamond discs. Since the top layer of the track is removed in this procedure, the macrotexture in positive z-direction is replaced by microtexture and the remaining (macro-)roughness is mostly in negative direction. This is illustrated in Figure 9 .

As can be seen in Figure 10 and Table 4, the mean profile depth varies between $0.7 \mathrm{~mm}$, for reference road surface (track 6 ), and $1.5 \mathrm{~mm}$, for the porous road surface with a large stone or ag- 


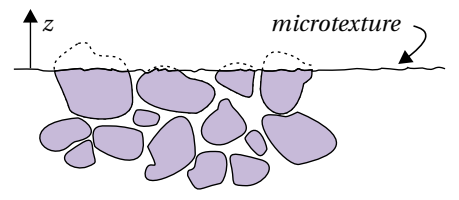

Figure 9: Illustration of road surface after grinding, such that macrotexture in positive $\mathrm{z}$-direction is replaced by microtexture.

gregate size (track 3 ). The variation in texture $z_{\text {rms }}$ follows the same trends as the MPD values of the test tracks.

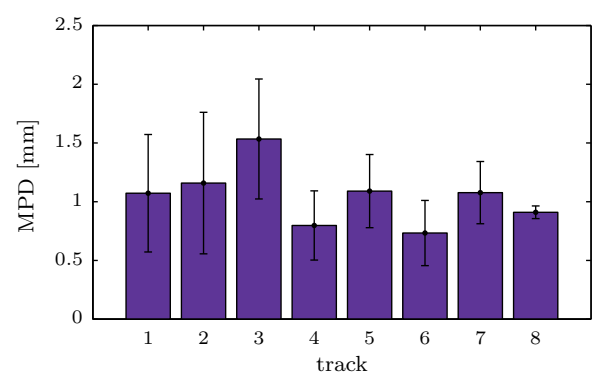

Figure 10: Mean profile depth (MPD) of the test tracks, the error bar indicates \pm 1 S.D.

Figure 11 shows the texture level for all tracks. Here, one can see that the texture level is large for the road surfaces with a large MPD and large variation in texture height, which are listed in Table 4. Furthermore, the peak value of the texture level is related to the maximum stone sizes in the mixture of the asphalt concrete. However, the direct relation between the maximum stone size and the wavelength of the maximum texture level as described by [25] is not seen in this data. Furthermore, the increase in the mean profile depth due to a larger stone size, as expected [25, 32], is also not clear from this data. Both effects could be explained by the bitumen removal procedure, since the (positive) macro roughness of these test tracks is removed.

As can be seen in Figure 12, the tracks with a higher porosity or air void content have larger values for the MPD and texture variation. For the porous road surfaces applies that the open pores at the surface contribute to a higher sur-

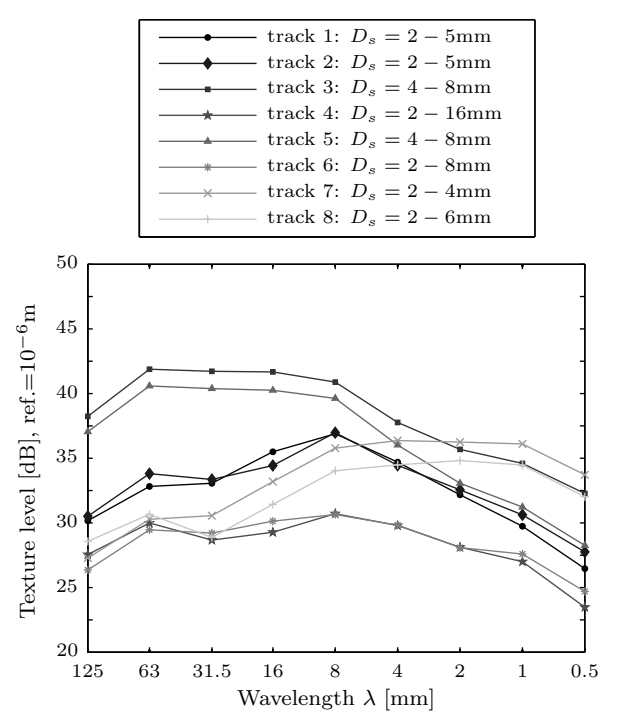

Figure 11: Texture profile level of all tracks.

face texture. This is also seen by [25]. Furthermore, the material of the stones or aggregates in the asphalt mixture can influence the surface texture. It is said that broken Bestone (used in track 1, 2, 3 and 8) has more rounded edges than some other materials, which would lead to a lower surface texture. This could explain why track 7 has a smaller surface texture than track 8 , while the stone size is almost identical.

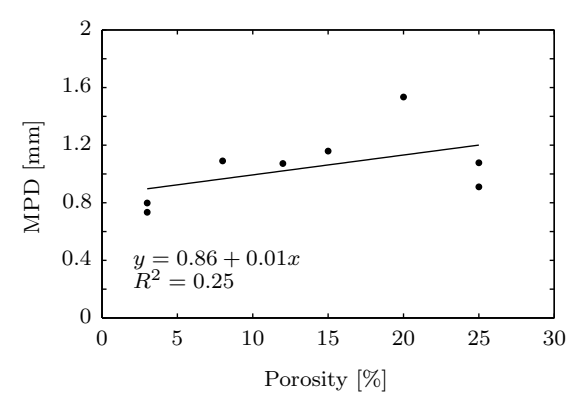

Figure 12: Relation between MPD and porosity or air void content. 


\begin{tabular}{lcccccccc}
\hline & Track 1 & Track 2 & Track 3 & Track 4 & Track 5 & Track 6 & Track 7 & Track 8 \\
\hline MPD $[\mathrm{mm}]$ & 1.1 & 1.2 & 1.5 & 0.8 & 1.1 & 0.7 & 1.1 & 0.9 \\
$z_{\text {rms }}[\mathrm{mm}]$ & 0.6 & 0.7 & 1.4 & 0.4 & 1.0 & 0.4 & 1.0 & 0.8 \\
\hline
\end{tabular}

Table 4: Road roughness parameters of the test tracks, where MPD is the mean profile depth and $z_{\text {rms }}$ is the variation in texture height, according to the definitions in ISO 13473-2:2002(en) [4].

\subsection{Sound absorption coefficient}

To determine the sound absorption of road surfaces, different methods can be used, like the impedance tube method (in-situ [5] or in lab [2]) and the extended surface method [3]. A discussion of these different methods can be found in [30].

For this research, we measured the sound absorption coefficient of the test tracks with two different measurement methods: (a) the impedance tube method and (b) a novel measurement technique using a small cubic microphone array.

The sound absorption coefficient for normal incidence is measured in-situ for all road surfaces with an impedance tube. Since tire/road noise will radiate noise mostly in oblique directions, the sound absorption coefficient for oblique incidence is also measured for three of the tracks, with a small cubic microphone array [22-24]. These measurements have been performed in an anechoic room at the University of Twente, using small cylindrical slabs of the test tracks.

\subsubsection{Impedance tube}

The sound absorption coefficient is measured following the method described in [34]. The open end of the tube was positioned on the road surface, using a flange sealed with clay. The sound absorption coefficient is measured at ten positions equally spaced in longitudinal direction of the test track. The variation in the other direction is also measured and has a good agreement with the measurements in the longitudinal direction. The repeatability of the measurements was tested for a number of the tracks and measurement locations.

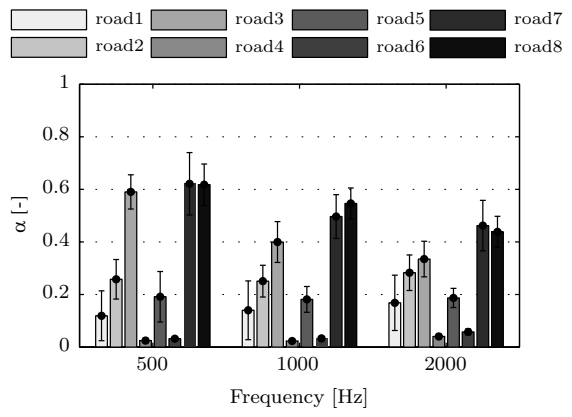

Figure 13: Sound absorption coefficient measured with impedance tube, errorbar indicates the \pm 1 S.D. measurement uncertainty.

The averaged sound absorption coefficient per track is determined in the continuous frequency spectrum, but for the purpose of this paper, only the results in the octave bands of $500 \mathrm{~Hz}, 1000 \mathrm{~Hz}$ and $2000 \mathrm{~Hz}$ are considered. These are shown in Figure 13 and listed in Table 6 in Appendix B. From this figure can be seen that there are four tracks with a sound absorption coefficient of more than $20 \%$ in all octave bands: track 2, 3, 7 and 8 . These road surfaces are referred to as open porous road surfaces, the other four tracks are referred to as dense road surfaces in the remainder of this paper.

\subsubsection{Cubic microphone array}

The sound absorption coefficient for oblique incidence is measured with a small threedimensional, cubic microphone array, containing 8 microphones, therefore called the $8 p$ probe. The design of the probe, the measurement technique and the measurement uncertainties are explained in more detail by [15, 2224]. The measurements are performed in an anechoic room using small cylindrical slabs. The slabs were taken from the test area using a $300 \mathrm{~mm}$ bore containing the track surface and 
a part of the (sound hard) fundament of the road.

The sound absorption coefficient for oblique incidence in octave bands for the all three tracks is shown in Figure 14 and listed in Table 7 in Appendix B. These measurements show the influence of oblique incident sound waves on the sound absorption coefficient. More details about these measurements are given in Appendix B. For both track 7 and 8, the largest sound absorption coefficient for an angle of incidence of $\theta=0^{\circ}$ and $\theta=30^{\circ}$ is in the $1000 \mathrm{~Hz}$ octave band, while for a larger angle of incidence, the maximum sound absorption coefficient is seen in the $2000 \mathrm{~Hz}$ band. This corresponds with the shift of the peak in the sound absorption coefficient to higher frequencies as seen in the simulations, in Section 3.1.

Note that the results for normal incidence $(\theta=$ $0^{\circ}$ ) are different than the results when using the impedance tube technique. When measuring the sound absorption coefficient with the $8 \mathrm{p}$ probe, the entire sound field around the slabs is measured. For the impedance tube technique, the sound absorption of the tracks is measured in-situ, which causes leakage of the sound to the area around the tube [30].

\subsection{Sound radiation and noise levels}

The focus of this paper is to show the relations between the tire and road characteristics and noise radiation, measured during closeproximity (CPX) measurements and controlled pass-by measurements. More about the measurement method for CPX and CPB can be found in the Appendices $\mathrm{C}$ and $\mathrm{D}$.

\subsubsection{Sound radiation}

The root mean square (rms) value of the sound pressure level (SPL) measured during the CPX measurements of the test tracks in combination with the three tires is listed in Table 5. Three different target speeds were used during these measurements and a single, interpolated SPL $L_{\mathrm{rms}}$ is derived, using a logarithmic regression
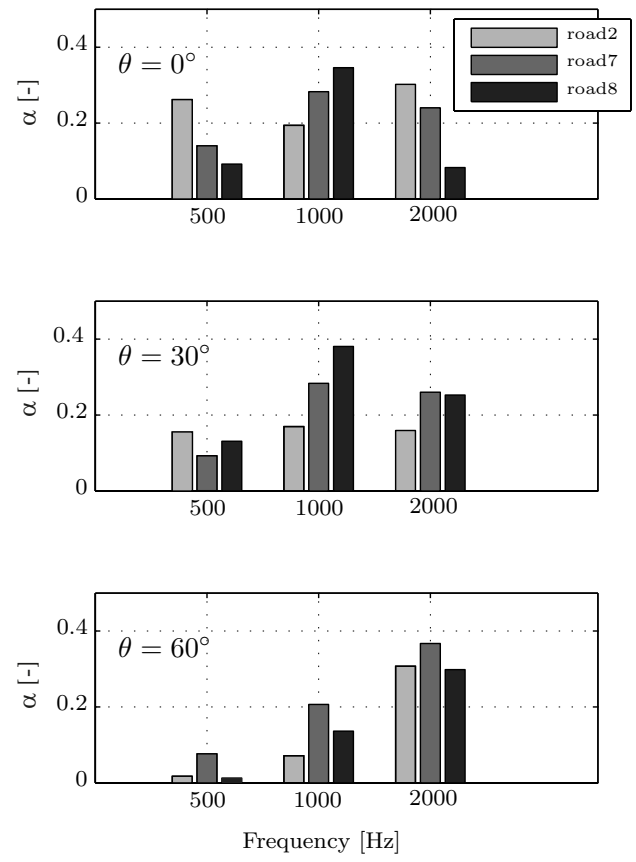

Figure 14: Sound absorption coefficient measured with 8p-probe.

over all measurements: $L_{\mathrm{rms}}=14 \log V-25$. The SPL given in this paper are for a velocity of $V=80 \mathrm{~km} / \mathrm{h}$. This correction for velocity is discussed in more detail in Appendix C.

\begin{tabular}{cccc}
\hline & Cento & SRTT & Slick \\
\hline Track 1 & $88.3 \pm 0.6$ & $90.2 \pm 0.4$ & $85.1 \pm 0.5$ \\
Track 2 & $85.8 \pm 0.8$ & $87.7 \pm 1.1$ & $82.3 \pm 0.5$ \\
Track 3 & $89.0 \pm 1.4$ & $89.5 \pm 2.1$ & $86.0 \pm 0.9$ \\
Track 4 & $90.9 \pm 0.3$ & $92.6 \pm 0.8$ & $87.2 \pm 2.1$ \\
Track 5 & $89.5 \pm 0.3$ & $90.5 \pm 0.3$ & $87.1 \pm 0.7$ \\
Track 6 & $90.3 \pm 0.0$ & $92.3 \pm 0.8$ & $87.2 \pm 1.8$ \\
Track 7 & $84.6 \pm 0.3$ & $86.4 \pm 0.2$ & $81.9 \pm 0.6$ \\
Track 8 & $85.4 \pm 0.9$ & $88.2 \pm 0.5$ & $83.0 \pm 0.4$ \\
\hline
\end{tabular}

Table 5: RMS values for SPL in $\mathrm{dB}(\mathrm{A})$ measured using an open CPX measurement device attached to the car, the results are corrected for velocity.

An overview of the sound reduction per tire/road combination is given in Figure 15. The sound reduction is based on the SPL compared 
to the reference tire, SRTT, on the reference road surface, ISO/R117, yielding:

$$
\Delta \mathrm{SPL}=\mathrm{rms}_{\mathrm{SRTT}, 6}-\mathrm{rms}_{i_{\text {tire }}, k_{\text {track }}}
$$

where $\triangle \mathrm{SPL}$ is the sound reduction in $\mathrm{dB}(\mathrm{A}), \mathrm{rms}_{\mathrm{SRTT}, 6}$ the reference rms value, and $\mathrm{rms}_{i_{\text {tire }}, k_{\text {track }}}$ the rms value of tire $i$ on track $k$. The continuous spectrum of this reference tire/road combination is shown in Figure 16. Here, the highest sound pressure level is seen between $800 \mathrm{~Hz}$ and $1200 \mathrm{~Hz}$, which is as expected for passenger car tire/road noise.

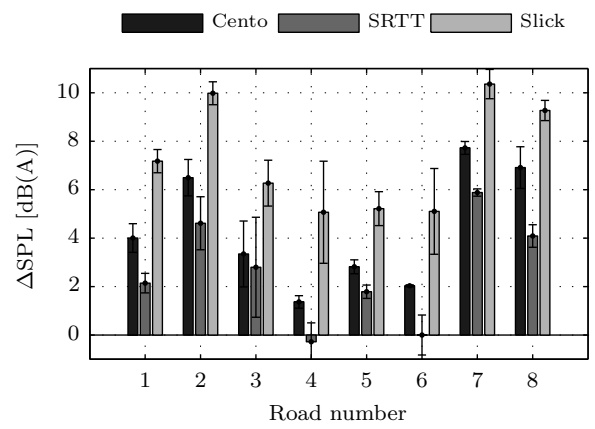

Figure 15: Sound reduction per road for CPX measurements, compared to SRTT on track 6, ISO/R117. The error bar indicates $\mathrm{a} \pm 1$ standard deviation.

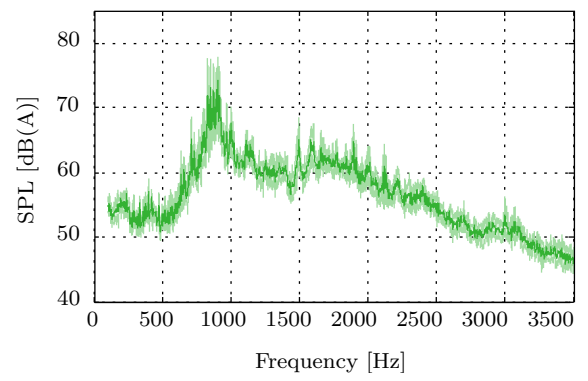

Figure 16: Continuous spectrum density (CPX measurement) of SRTT on track 6, ISO/R117. The shaded region indicates $\mathrm{a} \pm 1$ standard deviation.

Figure 15 shows that sound reduction depends on both tire and road surface. The difference in sound reduction between the SRTT and slick is about $5 \mathrm{~dB}(\mathrm{~A})$ for any track, thus appearing to be independent of the road surface. Overall, the reduction for the SRTT is smallest or even negative. The slick tire performs best on all tracks. This is expected since there is no tire profile to induce excitation mechanisms such as air pumping and vibrations of the tread blocks. Furthermore, from Figure 15 can be concluded that the road surfaces with large porosity (track $2,3,7$, and 8 ), have the largest sound reduction, up to $6 \mathrm{~dB}(\mathrm{~A})$ for the reference tire on the track with the best performance. However, track 3 performs less than the other open road surfaces, this is discussed in more detail later on.

The sound reduction per octave band for the CPX measurements is summarized in Table 9 in Appendix C. Figure 17 shows the sound reduction for the road surfaces with a sound absorption above $20 \%$. The sound reduction is highest in the $1000 \mathrm{~Hz}$ octave band, the same band where the sound radiation is largest (Figure 16). The reduction is upto $13 \mathrm{~dB}(\mathrm{~A})$ for the best tire/road combinations. However, these results are obtained for the slick tire, which is a tire not allowed in normal traffic. But also for the SRTT the results for these open road surfaces show a reduction of at least $5 \mathrm{~dB}(\mathrm{~A})$. It can also be seen in Figure 17 that the differences in sound reduction between the Cento and the SRTT are larger for higher frequencies, indicating that for these frequencies the tire properties are more important than the road properties.

Furthermore, the results in Figure 17 show that the sound reduction in the $500 \mathrm{~Hz}$ octave band is small or even negative for track 3 . Note that the sound absorption coefficient for this track is one of the highest in the $500 \mathrm{~Hz}$ band and similar to the sound absorption coefficient of track 7 and 8 (as listed in Table 6). This indicates that other mechanisms are more important in this octave band. When considering the road roughness and stone size (Table 3 ) of this track, the surface texture of this track is larger than for the other tracks which can explain the larger sound radiation.

The performance of track 3 is better in the other two octave bands, which indicates that in these octave bands the sound absorption coefficient 
is more important than the other road properties. However, the other tracks with a large sound absorption coefficient still perform better than track 3 .

The same trends are visible in the sound reduction per octave band based on the CPB measurements, which are summarized in Table 12 in Appendix D.
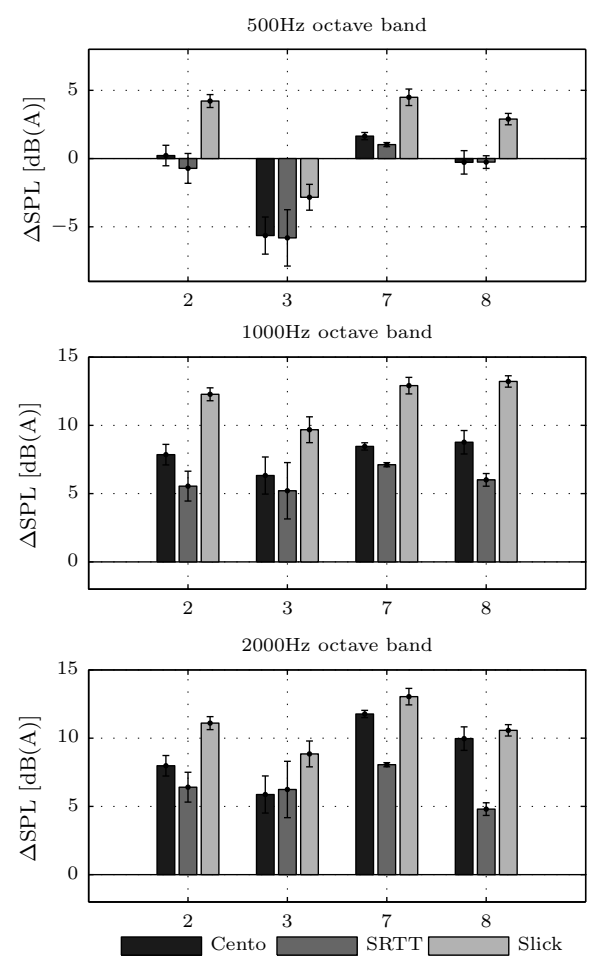

Figure 17: Sound reduction per octave band (CPX measurements), for track 2 , track 3 and the prototype tracks. The error bar indicates a \pm 1 standard deviation.

\subsubsection{Correlation between CPX and CPB measurements}

As previous studies have indicated $[10,16]$, there is a correlation between CPX and CPB measurements that depends on the velocity of the vehicle, the road surface and the frequency [16]. Here, we found similar results, shown in
Figure 18. The relation is considered per octave band and it is found that this relation not only depends on the frequency of the noise, but also on the porosity of the road surfaces. The results, when making a distinction between the open roads and the dense road surfaces (sound absorption less than $20 \%$ ), show that for higher frequencies the differences become more relevant. However, we used primarly the CPX results in the remainder of this paper, since the measurement accuracy is larger.

\subsubsection{Performance of prototype tracks}

In Section 3, the design process of the two prototype tracks is discussed, which have been based on literature, measurements and predictive models.

The performance of track 7 and 8 , the two prototype tracks, is good. However, the performance of track 2 is similar, as shown in Figure 17, while the sound absorption coefficient of this track is much lower. The sound reduction is largest in the $1000 \mathrm{~Hz}$ and $2000 \mathrm{~Hz}$ octave band, which is expected when considering the combination of sound radiation and sound absorption coefficient in these bands.

The sound reduction is high for both the threaded tires (the SRTT and Cento) is the highest for track 7, up to $L_{\mathrm{rms}}=6 \mathrm{~dB}(\mathrm{~A})$ for the SRTT. The sound absorption coefficient and porosity for track 7 and 8 are similar. Also, the road roughness parameters and the stone sizes used in both roads are almost similar. The largest difference between the two tracks is the layer thickness. The large layer thickness for track 7 shows in the largest sound reduction in the $500 \mathrm{~Hz}$ octave band, although the differences are small.

\section{Discussion of tire and road param- eters}

The main goal of this paper is to find the influence of both tire and road properties on the sound radiation. Much research has been done 

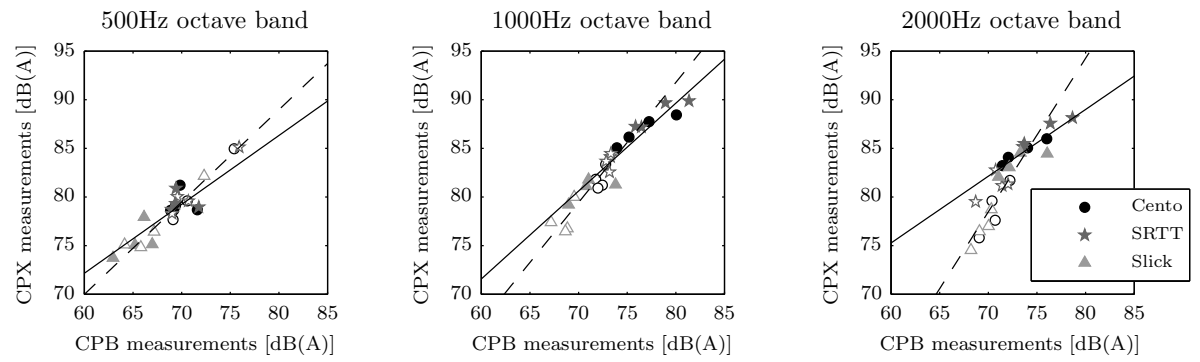

Figure 18: Correlation CPX and CPB per octave band. The open markers indicate the SPL for the open road surfaces and the closed markers indicate the SPL for the dense road surfaces. The dashed line indicates the trend in measurement results for the open roads and the solid line indicates the trend for the dense road surfaces. The tires are indicated by the different markers.

to find the influence of road parameters on the sound absorption coefficient or the sound radiation [20, 21, 26, 27, 31-33]. However, considerably less is written about the influence of tire parameters on the noise level $[9,18,33]$. Furthermore, a discussion of the combination between road and tire parameters is difficult to find. Most literature is focused on tire/road noise with respect to vehicle types and statistical pass-by measurements without making a distinction between the type or design of the tires.

In this section, the influence of the tire and road parameters on the sound radiation is discussed. This section also discusses the influence of some of the road parameters on the sound absorption coefficient.

\subsection{Tire parameters}

The tire parameters have been defined in Section 2.1.

\subsubsection{Pattern continuity}

A clear trend is visible when looking at the results in Figure 19. A higher pattern continuity correlates with a lower sound radiation, which is according to expectations, since a higher pattern continuity leads to less pressure variation in the footprint and thus to less vibrations of the tread blocks and tire.

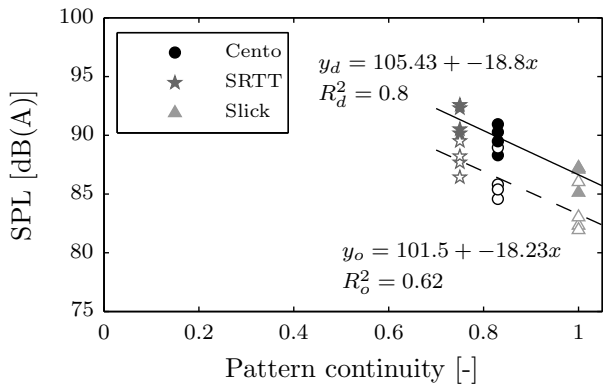

Figure 19: Influence of pattern continuity on the sound radiation (CPX). The open markers indicate the open road surfaces and the colored markers the dense road surfaces. The solid line is the trend for the dense road surfaces, the dashed line for the open road surfaces.

\subsubsection{True contact ratio}

The only combined tire/road parameter that is considered in this paper is the true contact ratio. The true contact ratio is listed in Table 2 for all combinations of tires and road surfaces used in this study. This parameter is zero for the slick tire on a road surface with no porosity. For the tested tire/road combinations the smallest value for the true contact ratio is found for the slick on the reference road surface (track 6). The maximal values for true contact ratio are found for the combinations of the tires with profile and the prototype tracks, having a large porosity.

Figure 20 shows that the general trend for all data points indicate that a lower true contact 


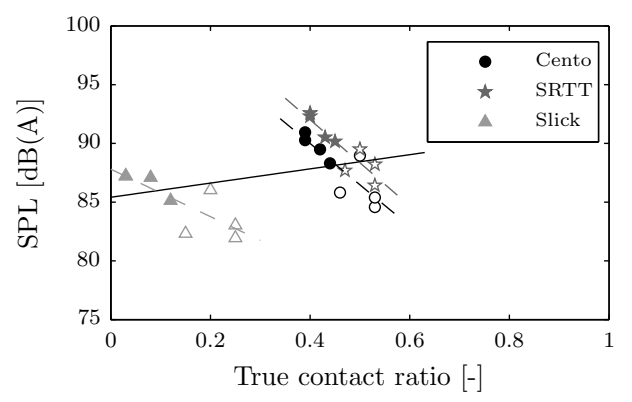

Figure 20: Influence of true contact area $R_{\mathrm{tc}}$ on the sound radiation (CPX). The open markers indicate the open road surfaces and the colored markers the dense road surfaces. The solid line shows the trend for all the data points, the dashed lines indicate the trends per tire.

area is beneficial for the sound reduction. However, when looking at the separate tires, the slope of the trend is reversed, as indicated with the dashed lines in Figure 20. The variation of the sound radiation for the same tire and a different road surface is up to $6 \mathrm{~dB}(\mathrm{~A})$. Looking at the results per tire, the most silent combination of tire and road has the largest value for the true contact ratio. However, there are more tire and road combinations having the same or almost the same true contact ratio, but a larger SPL. This indicates that other effects depending on the tire or the road, are more dominant than the true contact area of the tire/road contact.

\subsection{Road parameters}

The influence of all road design parameters on the sound radiation are discussed below. For some of the road parameters, the influence on the sound absorption coefficient is also considered. The relation between the sound absorption and the sound radiation has already been discussed in Section 4.3.

\subsubsection{Influence of porosity}

The first road parameter is the porosity of the road. The open pores in a porous road surface are important for the sound absorption, because of the viscothermal energy dissipation inside the (small) pores. The porosity of the road surface depends on the air void content and the connectivity of the air voids in the asphalt layer, since sound waves can only travel through the air voids connected to each other and to the surface of the road. Li et al. [25] show that there is a relation between the air void content and the degree of connectivity. For mixtures with an air void content below $12 \%$, the connectivity is poor and there are almost no pores connecting from top to bottom, resulting in a low volume porosity. For mixtures with an air void content above $20 \%$, the connectivity is very high and pores can stretch through the entire layer height, which results in a volume porosity almost equal to the air void content. Parameters that influence the air void content and the connectivity are the stone size and variation within the mixture, the type of bitumen and the compression of the mixture during construction.

The values for the porosity listed in Table 3 are the design values for the tracks. The final volume porosity, air void content and degree of connectivity of the constructed tracks is not measured.

The relation between the porosity and the amplitude of the first peak in the sound absorption value is shown in Figure 21. The relation found in these measurements corresponds very well with the experimental results found by $\mathrm{Li}$ et al. [25] between the air void contents and the first peak value of the sound absorption coefficient. The relation between the porosity and the rms value of the sound radiation is shown in Figure 22. The trend line shows the relation for all data points. The sound radiation is lower for a road surface with a higher porosity, this relation is also found in the measurements by Liao et al. [27].

\subsubsection{Influence of layer thickness}

The relation between layer thickness and the sound radiation is considered in octave bands. Again, a distinction is made between the open and dense road surfaces based on porosity. The results are shown in Figure 23. It is expected 


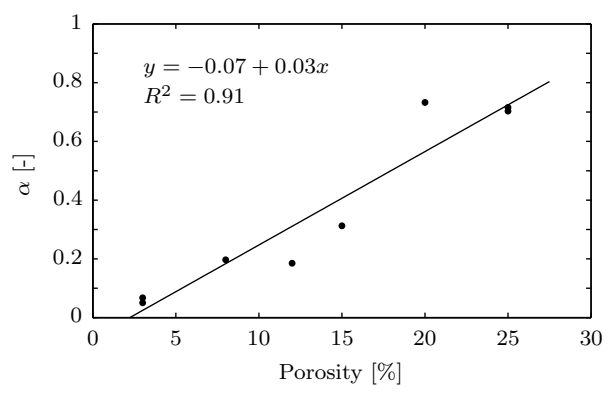

Figure 21: Relation between the porosity and the amplitude of the first peak of the sound absorption coefficient.

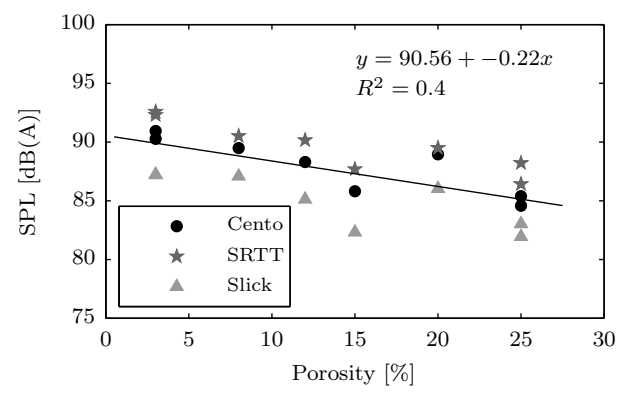

Figure 22: Relation between the porosity and the sound radiation (CPX).

that the influence of the layer thickness is most visible for the open road surfaces in lowest frequency band, since the longer wavelengths require a larger (porous) layer thickness to be absorbed. Track 7 has the largest layer thickness, due to the double layered asphalt concrete and is one of the more silent roads in the higher frequency bands. However, the differences are small in the $500 \mathrm{~Hz}$ band.

More general, Figure 23 shows that the open road surfaces perform better in the $1000 \mathrm{~Hz}$ octave band and especially in the $2000 \mathrm{~Hz}$ octave band. The trend lines indicated in Figure 23 show a positive influence of the increasing layer height for the $1000 \mathrm{~Hz}$ and $2000 \mathrm{~Hz}$ octave band. Note that the correlation coefficient $R^{2}$ is very low for all three trend lines. However, the trends seen in Figure 23 point to the earlier conclusion that the layer thickness of the road surface is more important for the higher frequencies.

\subsubsection{Influence of stone size and variation}

Another road design parameter is the stone or aggregate size and the variation in stone sizes in the asphalt mixture. Larger stone sizes will give wider pores and therefore, the loss mechanisms due to viscous effects will be less. On the other hand, smaller stones will give smaller sized pores, which will obstructed more easily by the bitumen during construction or the dirt during its lifetime. Li et al. [25], discusses the influence of the stone size within the mixture on the sound absorption coefficient. They found an increase in the amplitude of the first peak in the sound absorption coefficient for an increasing stone size. The results found here are correlated badly. However, we only considered 8 road surfaces in this research, while Li et al. [25] has looked at about 25 different road surfaces.

Furthermore, the stone size has an influence on the road roughness and therefore, on the excitation mechanisms of tire/road noise. In general, larger stones will correspond with a larger road roughness, which has a negative effect on the sound excitation, especially for lower frequencies. For higher frequencies, the increased road roughness will influence effects such as air pumping and stick-slip of the tire tread blocks [32].

Figure 24 shows the influence of the largest stone sizes within the mixture on the rms value of the SPL. From this figure it is clear that a larger maximum stone size results in a higher sound radiation. This figure also shows that for similar stone size, the SPL for the open road surfaces is almost $5 \mathrm{~dB}(\mathrm{~A})$ lower.

Furthermore, the variation in stone sizes within the asphalt mixture is important. It is expected that for a large variation in stone size, the air void content inside the road surfaces, and thus the porosity, will be lower, since the larger air pockets and pores can be filled with the smaller stone fractions. However, this is not researched in this paper. 

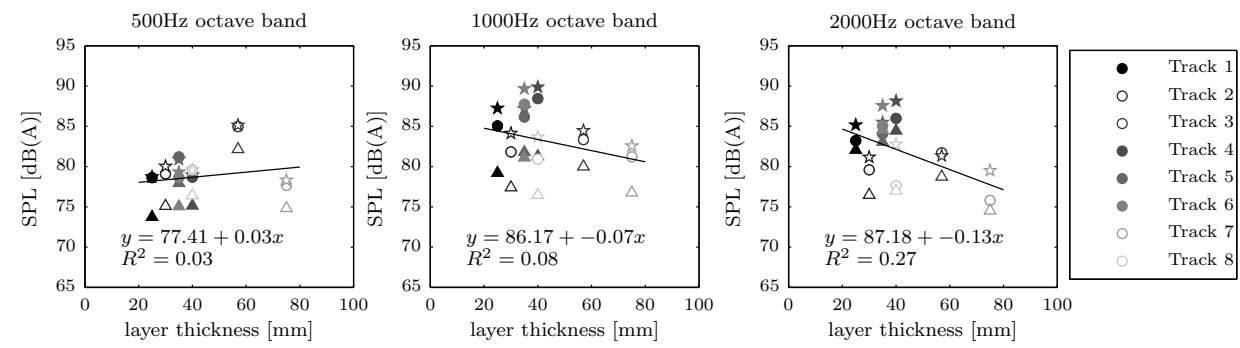

Figure 23: Influence of layer thickness on sound radiation (CPX). The markers indicate the three tires, where the circle is the Cento, the star is the SRTT and the triangle the slick tire. The open markers indicate the open road surfaces and the colored markers the dense road surfaces. The trend line for all data points is indicated in each plot.

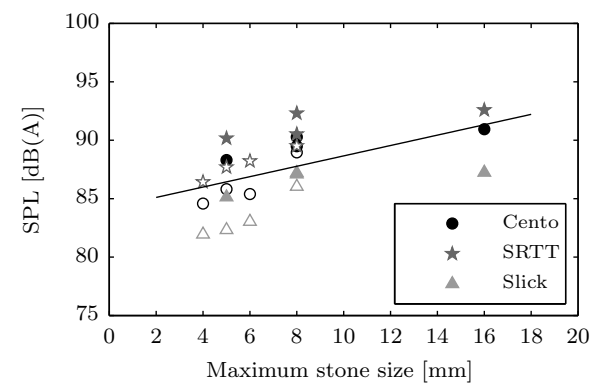

Figure 24: Influence maximum dimensions of stone within the asphalt mixture on the sound radiation (CPX). The open markers represent the open road surfaces and the colored markers the dense road surfaces.

\subsubsection{Influence of road roughness}

The expected influence of the road roughness has been described in the previous section. Two road roughness parameters are discussed here, the mean profile depth (MPD) and the variation in texture height $\left(z_{\mathrm{rms}}\right)$, as listed in Table 3 .

Figure 25 shows the influence of the MPD on the sound pressure level (measured with the open CPX device) per octave band. A distinction is made between open road surfaces and dense road surfaces.

The results correspond with the correlations found by [27]. For the dense road surfaces is found that in the $500 \mathrm{~Hz}$ band, an increasing surface texture results in an increasing sound pres- sure and that for the higher frequencies, an increasing surface texture results in a decreasing sound pressure level. The trends for the open road surfaces show that an increase in the texture results in an increase in the sound pressure level. The slope of this increase decreases with an increase in frequency. In [27] is found that for even higher frequencies $(f=2500 \mathrm{~Hz})$, the slope becomes negative, which means that an increase in surface texture results in a decrease in the sound pressure level.

This could be explained by the fact that for a road surface with a higher roughness and a large surface porosity it will be more difficult to trap air in between the profile of the tires (commonly called air-pumping), an effect that is more important for high frequencies [32]. However, due to the bitumen removal procedure, it was expected that this relation would not be found here.

The influence of the variation in texture height on the sound radiation are similar to that of the mean profile depth, since both road roughness parameters follow the same trends.

\section{Conclusions}

In this paper, several tire and road parameters are considered to find the relation between these parameters and the sound radiation, such that the tire and road surface can be optimised together in the design phase of both tire manufacturing and road construction. Eight test 

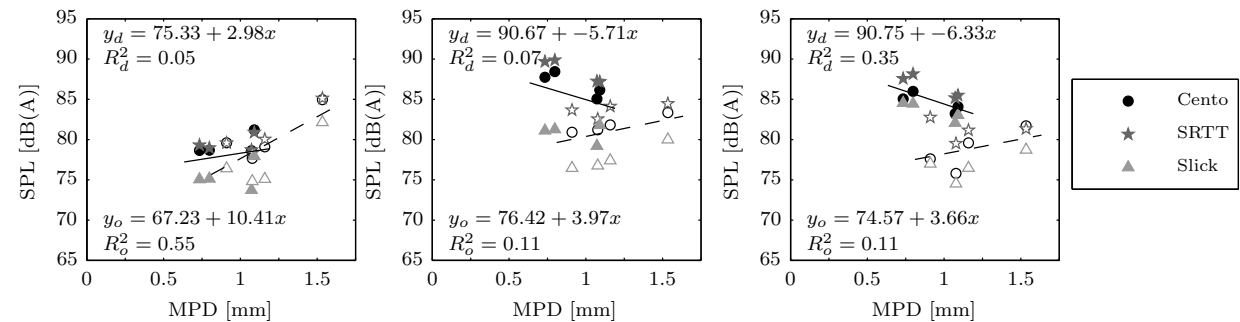

Figure 25: Influence of road roughness (MPD) on SPL measured by CPX device. The open markers represent the open road surfaces and the colored markers the dense road surfaces.

tracks were constructed at a local, rural airport in the east of The Netherlands, Airport Twente, within the project 'Silent and Safe Road Traffic'. Two of these test tracks were developed based on measurements, predictive models and design tools, all carried out or developed within this project.

The performance of these two prototype tracks was according to the expectations. Both tracks were designed to have a high sound absorption coefficient in the $1000 \mathrm{~Hz}$ octave band, especially for oblique incidence. The latter was tested with measurements in the lab and these results showed a good sound absorption coefficient for the larger angles of incidence. Furthermore, the sound reduction of the rms value of the SPL of these two road surfaces was up to $10 \mathrm{~dB}(\mathrm{~A})$ for a slick tire and more than $5 \mathrm{~dB}(\mathrm{~A})$ for the SRTT, both compared to the performance of the SRTT on the reference track, the ISO/R117.

Some general trends that followed from all the measurements performed at the test tracks are:

- There is a correlation between CPX and CPB measurements. However, this relation depends on the frequency and for higher frequencies the relation becomes increasingly depending on the sound absorption coefficient of the road surfaces.

- The frequency of the first peak in the sound absorption coefficient shifts to higher frequencies for an increasing angle of incidence. The amplitude of the peak decreases for an increasing angle of incidence.

- A tire tread design with a higher continuity (less variation in contact area during rolling) corresponds with a lower sound radiation.

- A conclusive relation between the air ratio of the tire tread $\left(1-S_{\text {tread }} / S_{\text {total }}\right)$, porosity of the road surface and the sound radiation is not found.

- A bitumen removal procedure like grinding replaces the macrotexture for microtexture, and reduces the sound radiation.

- The sound reduction depends on both the tire and the road surfaces, and on the frequency. The differences in sound reduction per tire for the same track are larger in the $1000 \mathrm{~Hz}$ and $2000 \mathrm{~Hz}$ octave band, an indication that for higher frequencies the tire properties are becoming increasingly important.

Furthermore, some of the results found in this research are consistent with conclusions found in earlier literature:

- A higher porosity or air void content leads to a higher sound absorption coefficient and lower sound radiation.

- For porous road surfaces, a larger layer thickness corresponds with a lower sound radiation.

- The surface texture of the road surfaces, determined by the road roughness and stone size, is mostly important for the sound radiation in the $500 \mathrm{~Hz}$ octave band. 
A larger surface texture relates to more sound radiation, which is assumed to be related to the excitation mechanisms of the tire/road contact.

- A larger stone or aggregate size corresponds with a larger sound radiation.

\section{Acknowledgements}

This project is carried out in the framework of the innovation program 'GO Gebundelde Innovatiekracht', funded by the 'European Regional Development Fund', 'Regio Twente' and 'Provincie Overijssel'. The project partners are Apollo Tyres Global R\&D, University of Twente (Tire-Road Consortium), Reef Infra, STEMMER IMAGING and the Provincie Gelderland, and their support is gratefully acknowledged.

\section{Appendix A: Properties of test tracks}

The properties of the 8 different test tracks at Airport Twente are summarized below.

\section{Track 1: Deciville Extra Levensduur}

- Porosity: $12-13 \%$

- Stone size: $2 / 5 \mathrm{~mm}$

- Material stone: Bestone, $100 \%$ broken surface

- Layer height: $25 \mathrm{~mm}$

\section{Track 2: Deciville Extra Stil}

- Porosity: $15 \%$

- Stone size: $2 / 5 \mathrm{~mm}$

- Material stone: Bestone, $100 \%$ broken surface

- Layer height: $30 \mathrm{~mm}$

\section{Track 3: OPA 8}

- Porosity: $20-21 \%$

- Stone size: $4 / 8 \mathrm{~mm}$
- Material stone: Bestone, $100 \%$ broken surface

- Layer height: $57 \mathrm{~mm}$

\section{Track 4: DAB}

- Porosity: $3-4 \%$

- Stone size: $4 / 8 \mathrm{~mm}, 8 / 11 \mathrm{~mm}, 11 / 16 \mathrm{~mm}$

- Material stone: Moräne, >95\% broken surface

- Layer height: $40 \mathrm{~mm}$

Track 5: Stil Mastiek

- Porosity: 6-10\%

- Stone size: $4 / 8 \mathrm{~mm}$

- Material stone: Graziet kleinhammer, $100 \%$ broken surface

- Layer height: $35 \mathrm{~mm}$

\section{Track 6: ISO/R117}

- Porosity: $3-4 \%$

- Stone size: $2 / 6 \mathrm{~mm}, 4 / 8 \mathrm{~mm}$

- Material stone: Graziet kleinhammer, $100 \%$ broken surface

- Layer height: $35 \mathrm{~mm}$

\section{Track 7: Twinlay}

- Porosity (top layer): $25 \%$

- Stone size (top layer): $2 / 4 \mathrm{~mm}$

- Material stone (top layer): Porfier, SFB 3100

- Layer height (top layer): $25 \mathrm{~mm}$

- Porosity (second layer): 25-26\%

- Stone size (second layer): 8/11

- Material stone (second layer):Moräne bitumen, 70/100

- Layer height (second layer): $50 \mathrm{~mm}$

\section{Track 8: OPA 6}

- Porosity: $25 \%$

- Stone size: $2 / 6 \mathrm{~mm}$

- Material stone: Bestone, SFB 3-100 
- Layer height: $40 \mathrm{~mm}$

\section{Appendix B: Sound absorption coeffi- cient}

In this appendix, the sound absorption coefficient per octave band is given for normal incident sound waves and for oblique incident sound waves.

\section{Impedance tube measurements for nor- mal incidence}

The sound absorption coefficient measured using the in-situ impedance tube technique is listed in Table 6. The results are shown in Figure 13 , where also the standard deviation is given.

\section{8p-probe measurements for oblique inci- dence}

The sound absorption coefficient measured with the $8 p$-probe measurement technique is listed in Table 7. The results are also shown in Figure 14.

Figure 26 shows the trends for the sound absorption coefficient three angles of incidence for track 7 measured with the $8 p$-probe and the results for normal incidence for the impedance tube measurement. Note that the measurement uncertainties of the 8p-probe measurements are not indicated in this figure, but are much larger than for the impedance tube, as can be found in [15]. The results obtained with the $8 \mathrm{p}$-probe are for normal incidence compared to the in-situ impedance tube measurements. The differences in results can be explained by the differences in method and environment. The measured sound absorption coefficient is the ratio between the active and incident sound waves, where the active sound waves are the difference between the reflected waves and the incident waves. When measuring in-situ with an impedance tube, the active sound waves do not only describe the sound

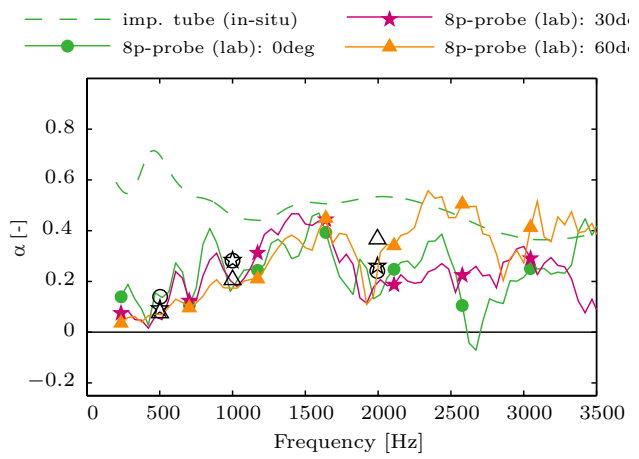

Figure 26: Measured sound absorption coefficient for track 7, for normal incidence, $\theta=30^{\circ}$ and $\theta=60^{\circ}$. The octave values are indicated by the open black markers.

waves absorbed by the porous road surface but also the sound waves that are transmitted inside and through the road surface and will surface outside the range of the impedance tube. While with the 8p-probe measurements, a sound field equally distributed over the complete road surface is used, the measured sound field is thus the active sound field for all incident, reflected and transmitted waves. Another major difference is the limited size of the samples measured in the lab with the 8p-probe. Therefore, the results shown in Figure 26 and Table 7 only show the trends of the sound absorption coefficient with increasing angle of incidence and are not representative for in-situ measurements with the $8 p$-probe. Figure 26 shows that the amplitude of the first absorption peak is lower for a higher angle of incidence, while the absorption coefficient at higher frequencies is larger for larger angles of incidence. Furthermore, a shift in frequency can be detected. Both effects are similar to the model results shown in Figure 7.

\section{Appendix C: CPX measurement meth- ods}

The CPX results presented in this paper are obtained with the measurement device as shown in Figure 27. The microphones of this device are in open air and the distance between the 


\begin{tabular}{ccccccccc}
\hline & Track 1 & Track 2 & Track 3 & Track 4 & Track 5 & Track 6 & Track 7 & Track 8 \\
\hline $500 \mathrm{~Hz}$ & 0.12 & 0.26 & 0.59 & 0.02 & 0.19 & 0.03 & 0.62 & 0.62 \\
$1000 \mathrm{~Hz}$ & 0.14 & 0.25 & 0.40 & 0.02 & 0.18 & 0.03 & 0.50 & 0.55 \\
$2000 \mathrm{~Hz}$ & 0.17 & 0.28 & 0.33 & 0.04 & 0.19 & 0.06 & 0.46 & 0.44 \\
\hline
\end{tabular}

Table 6: Sound absorption coefficient [-] per octave band.

\begin{tabular}{cccccccccc}
\hline & \multicolumn{3}{c}{ Track 2 } & \multicolumn{3}{c}{ Track 7 } & \multicolumn{3}{c}{ Track 8 } \\
& $0^{\circ}$ & $30^{\circ}$ & $60^{\circ}$ & $0^{\circ}$ & $30^{\circ}$ & $60^{\circ}$ & $0^{\circ}$ & $30^{\circ}$ & $60^{\circ}$ \\
\hline $500 \mathrm{~Hz}$ & 0.26 & 0.16 & 0.02 & 0.14 & 0.09 & 0.08 & 0.09 & 0.13 & 0.01 \\
$1000 \mathrm{~Hz}$ & 0.19 & 0.17 & 0.07 & 0.28 & 0.28 & 0.21 & 0.35 & 0.38 & 0.14 \\
$2000 \mathrm{~Hz}$ & 0.30 & 0.16 & 0.31 & 0.24 & 0.26 & 0.37 & 0.08 & 0.25 & 0.30 \\
\hline
\end{tabular}

Table 7: Sound absorption coefficient [-] for oblique incidence per octave band.

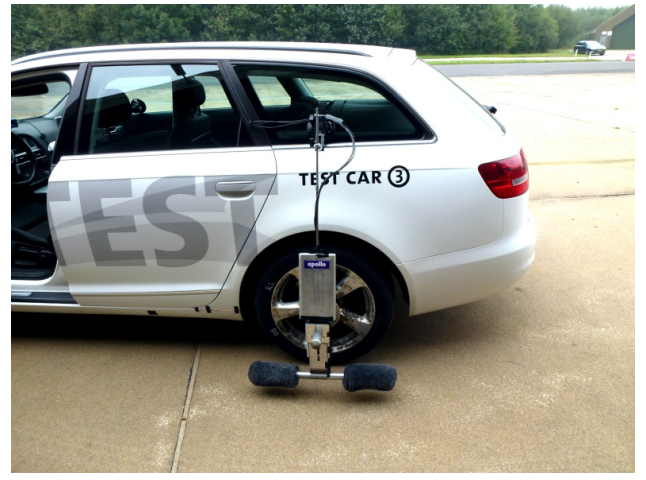

Figure 27: Measurement device used for CPX measurements.

microphones and the tires is larger than for a standardized CPX trailer. The relation between this (open) device and the more traditional CPX measurement trailer is not discussed or used here, for more information the reader is referred to [14].

\section{Velocity correction}

The CPX measurements are preformed on all 8 road surfaces, using three different tires, for 3 different speeds: $50 \mathrm{~km} / \mathrm{h}, 80 \mathrm{~km} / \mathrm{h}$ and $100 \mathrm{~km} / \mathrm{h}$. The results are taken together using a velocity correction, as described by [33, chap. 9]. The relation between the velocity and the A-weighted sound pressure level is according to:

$$
L=A+B \cdot \log (V)
$$

where $L$ is the Sound Pressure Level in $\mathrm{dB}(\mathrm{A})$, $A$ and $B$ are constant speed coefficient and $V$ is the speed in $\mathrm{km} / \mathrm{h}$. The coefficients $A$ and $B$ depend on the type of tires, cars, road surface and measurement method. In general, a smaller $A$ indicates a more silent tire, the coefficient $B$ indicates the slope of the relation between the speed and noise, a higher $B$ is a larger slope, thus the noise depends more on the speed.

Here, the relation is found combining all CPX measurements, and expressed per octave band, as listed in Table 8. In this paper, the CPX results are considered at a reference speed of $80 \mathrm{~km} / \mathrm{h}$.

\begin{tabular}{ccc}
\hline Octave band & A & B \\
\hline $500 \mathrm{~Hz}$ & 40.5 & 8.7 \\
$1000 \mathrm{~Hz}$ & 24.4 & 13.5 \\
$2000 \mathrm{~Hz}$ & 10.5 & 16.2 \\
\hline
\end{tabular}

Table 8: Velocity correction for CPX measurements, according to $L=A+B \cdot \log (V)$.

\section{Sound reduction}

The sound reduction is given for each tire/road combination, based on the reduction obtained 
compared to the reference tire, SRTT, on the reference road surface, ISO/R117, given by

$$
\Delta \mathrm{SPL}=\mathrm{rms}_{\mathrm{SRTT}, 6}-\mathrm{rms}_{i_{\text {tire }}, k_{\text {track }}}
$$

where $\triangle$ SPL is the sound reduction in $\mathrm{dB}(\mathrm{A}), \mathrm{rms}_{\mathrm{SRTT}, 6}$ the reference rms value, and $\mathrm{rms}_{i_{\text {tire }}, k_{\text {track }}}$ the rms value of tire $i$ on track $k$. The sound reduction for the rms value of the SPL is shown in Figure 15 and is listed in Table 9 for each octave band.

\section{Appendix D: CPB measurement meth- ods}

The pass-by measurements performed for this project are based on the controlled pass-by method, [33, page 305].

The noise is measured at three different microphone positions at $7.5 \mathrm{~m}$ from the center of the road. For this paper, only the results at a height of $3.0 \mathrm{~m}$ are used.

\section{Measurement method}

The Sound Pressure Level in $\mathrm{dB}(\mathrm{A})$ in the time domain is obtained by summation of the different frequency bands:

$$
L_{A, p}(t)=10 \log _{10}\left(\sum 10^{L_{A p, i} / 10}\right)
$$

The maximum value in the time domain $L_{A, \max }$ is determined and a small region of around this moment in time, $t_{\text {center }}$, is evaluated further. The width of this time region is chosen dependent on the velocity of the passing car, as listed in Table 10 .

\section{Velocity correction}

As for the CPX results, a logarithmic velocity regression relation in Equation4 is used to analyse the results together. The correlation per octave band is listed in Table 11.

\section{Sound reduction}

The sound reduction is given for each tire/road combination, based on the reduction obtained compared to the reference tire, SRTT, on the reference road surface, ISO/R117, given by

$$
\Delta \mathrm{SPL}=\mathrm{L}_{\mathrm{SRTT}, 6}-\mathrm{L}_{i_{\text {tire }}, k_{\text {track }}}
$$

where $\triangle \mathrm{SPL}$ is the sound reduction in $\mathrm{dB}(\mathrm{A})$, $\mathrm{L}_{\mathrm{SRTT}, 6}$ the reference $L_{\mathrm{A}, \max }$ value, and $\mathrm{L}_{i_{\text {tire }}, k_{\text {track }}}$ the $L_{\mathrm{A}, \max }$ value of tire $i$ on track $k$.

\section{References}

[1] ISO 11819-1:1997(en) Acoustic - Measurement of the influence of road surfaces on traffic noise - Part 1: Statistical Pass-By method, 1997.

[2] ISO 10534-2:1998(en) Acoustics - Determination of sound absorption coefficient and impedance in impedance tubes - part 2: Transfer function method, 1998.

[3] ISO 13472-1:2002 Acoustic - Measurement of sound absorption properties of road surfaces in situ - Part 1: Extended surface method, 2002.

[4] ISO 13473-2:2002(en) Characterization of pavement texture by use of surface profiles - Part 2: Terminology and basic requirements related to pavement texture profile analysis, 2002.

[5] ISO 13472-2:2010(en) Acoustics - Measurement of sound absorption properties of road surfaces in situ -Part 2: Spot method for reflective surfaces, 2010.

[6] ISO 11819-2:2017(en) Acoustic - Measurement of the Influence of road surfaces on traffic noise - Part 2: The close-proximity method, 2017.

[7] F. Anfosso-Lédée and Y. Pichaud. Temperature effect on tyre-road noise. Applied Acoustics, 68(1):1-16, 2007.

[8] K. Attenborough, I. Bashir, and S. Taherzadeh. Outdoor ground 


\begin{tabular}{ccccccccc}
\hline & Track 1 & Track 2 & Track 3 & Track 4 & Track 5 & Track 6 & Track 7 & Track 8 \\
\hline $\mathbf{5 0 0 H z}$ & & & & & & & & \\
Cento & 0.7 & 0.2 & -5.6 & 0.6 & -1.9 & 0.7 & 1.6 & -0.3 \\
SRTT & 0.6 & -0.7 & -5.8 & 0.4 & -1.6 & $\mathbf{0 . 0}$ & 1.0 & -0.3 \\
Slick & 5.6 & 4.2 & -2.8 & 4.2 & 1.4 & 4.3 & 4.5 & 2.9 \\
\hline $\mathbf{1 0 0 0 H z}$ & & & & & & & & \\
Cento & 4.6 & 7.9 & 6.3 & 1.2 & 3.5 & 1.9 & 8.5 & 8.8 \\
SRTT & 2.4 & 5.5 & 5.2 & -0.2 & 2.5 & $\mathbf{0 . 0}$ & 7.1 & 6.0 \\
Slick & 10.5 & 12.3 & 9.7 & 8.4 & 7.9 & 8.6 & 12.9 & 13.2 \\
\hline $\mathbf{2 0 0 0 H z}$ & & & & & & & & \\
Cento & 4.4 & 8.0 & 5.9 & 1.6 & 3.5 & 2.5 & 11.8 & 10.0 \\
SRTT & 2.4 & 6.4 & 6.2 & -0.6 & 2.1 & $\mathbf{0 . 0}$ & 8.1 & 4.8 \\
Slick & 5.5 & 11.1 & 8.8 & 3.1 & 4.5 & 3.0 & 13.0 & 10.6 \\
\hline
\end{tabular}

Table 9: Sound reduction, based on CPX measurement results per octave band in $\mathrm{dB}(\mathrm{A})$.

\begin{tabular}{cc}
\hline Velocity $[\mathrm{km} / \mathrm{h}]$ & $\Delta t[\mathrm{~s}]$ \\
\hline 50 & 1.0 \\
80 & 0.75 \\
100 & 0.5 \\
\hline
\end{tabular}

Table 10: Width of time segment used for further evaluation pass-by data.

\begin{tabular}{ccc}
\hline Octave band & A & B \\
\hline $500 \mathrm{~Hz}$ & 30.9 & 8.9 \\
$1000 \mathrm{~Hz}$ & 11.3 & 14.4 \\
$2000 \mathrm{~Hz}$ & 12.7 & 13.6 \\
\hline
\end{tabular}

Table 11: Velocity correction for CPB measurements, according to $L=A+B \cdot \log (V)$.

impedance models. J. Acoust. Soc. Am., 129:2806-2819, 2011.

[9] D. Bekke, Y. H. Wijnant, T. Weegerink, and A. de Boer. Tire-road noise: an experimental study of tire and road design parameters. In Noise Control for Quality of Life, pages 564-570. Austrian Noise Abatement Association, 92013.

[10] D. A. Bekke. Engineering tools for interior tyre tread pattern noise. PhD thesis, University of Twente, 2014.

[11] D. A. Bekke, Y. H. Wijnant, D. Schipper, B. Bobbink, G. Oude Lansink, and R. Stevens. Silent and Safe Roadtrafficproject: An optimization of the tyre-road interaction on noise and wet grip. In $\mathrm{Eu}$ ronoise 2015, pages 1345 - 1350, June 2015.

[12] M. Bezemer-Krijnen, Y. H. Wijnant, and A. de Boer. Three-dimensional modelling of sound absorption in porous asphalt pavement for oblique incident waves. In Euronoise 2015, pages 1007 - 1012, June 2015.

[13] M. Bezemer-Krijnen, Y. H. Wijnant, and A. de Boer. Predicting sound absorption of stacked spheres: combining an analytical and numerical approach. Acta Acustica united with Acustica, 102:971-983, August 2016.

[14] M. Bezemer-Krijnen, Y. H. Wijnant, and A. de Boer. Tyre-road noise measurements: influence of tyre tread and road characteristics. In Inter-noise 2016, 2016.

[15] M. Bezemer-Krijnen, Y. H. Wijnant, and A. de Boer. Prediction of sound absorption of stacked granular materials for oblique incident sound waves. Acta Acustica united with Acustica, 104(3):464-476, May/June 2018.

[16] J. Cesbron and P. Klein. Correlation between tyre/road noise levels measured by the coast-by and the close-proximity methods. Applied Acoustics, 126:36 - 46, 2017. 


\begin{tabular}{ccccccccc}
\hline & Track 1 & Track 2 & Track 3 & Track 4 & Track 5 & Track 6 & Track 7 & Track 8 \\
\hline $\mathbf{5 0 0 H z}$ & & & & & & & & \\
Cento & 0.5 & 0.0 & -6.0 & -2.2 & -0.4 & 0.2 & 0.3 & -1.2 \\
SRTT & 0.4 & -0.2 & -6.5 & -2.4 & -0.0 & $\mathbf{0 . 0}$ & 0.4 & -1.3 \\
Slick & 6.5 & 5.3 & -2.9 & 2.4 & 3.3 & 4.2 & 3.6 & 2.2 \\
\hline $\mathbf{1 0 0 0 H z}$ & & & & & & & & \\
Cento & 5.0 & 7.2 & 6.1 & -1.1 & 3.7 & 1.7 & 6.5 & 6.9 \\
SRTT & 3.1 & 5.6 & 5.6 & -2.4 & 2.5 & $\mathbf{0 . 0}$ & 5.8 & 6.1 \\
Slick & 10.0 & 11.8 & 9.4 & 5.1 & 7.9 & 8.1 & 10.1 & 10.3 \\
\hline $\mathbf{2 0 0 0 H z}$ & & & & & & & & \\
Cento & 4.9 & 6.0 & 4.2 & 0.4 & 4.3 & 2.3 & 7.3 & 5.7 \\
SRTT & 2.8 & 5.0 & 4.4 & -2.3 & 2.7 & $\mathbf{0 . 0}$ & 7.7 & 5.6 \\
Slick & 5.3 & 7.3 & 6.1 & 0.3 & 4.2 & 3.1 & 8.2 & 6.4 \\
\hline
\end{tabular}

Table 12: Sound reduction, based on CPB measurement results for each octave band in $\mathrm{dB}(\mathrm{A})$, at $h=3.0 \mathrm{~m}$.

[17] Y. Champoux and M. R. Stinson. On acoustical models for sound propagation in rigid frame porous materials and the influence of shape factors. J. Acoust. Soc. Am., 92:1120-1131, 1992.

[18] J. Eisenblaetter, S. J. Walsh, and V. V. Krylov. Air-related mechanisms of noise generation by solid rubber tyres with cavities. $A p$ plied Acoustics, 71(9):854-860, 2010.

[19] J. F. Hamet and M. Berengier. Acoustical characteristics of porous pavements: a new phenomenological model. In INTERNOISE 93: people versus noise, Leuven, Belgium, pages $641-646,1993$.

[20] M. Heckl. Tyre noise generation. Wear, 113(1):157-170, 1986.

[21] J. Kragh, B. Andersen, and J. Oddershede. Road surface texture: low noise and low rolling resistance: Cpx trailer comparison: Copenhagen 2009, 2010.

[22] E. R. Kuipers. Measuring sound absorption using local field assumptions. $\mathrm{PhD}$ thesis, University of Twente, 2013.

[23] E. R. Kuipers, Y. H. Wijnant, and A. de Boer. Measuring oblique incidence sound absorption using a local plane wave assumption. Acta Acustica united with Acustica, 100(2):205-214, 2014.
[24] E. R. Kuipers, Y. H. Wijnant, and A. de Boer. Measuring sound absorption: considerations on the measurement of the active acoustic power. Acta Acustica united with Acustica, 100(2):193 - 204, 2014.

[25] M. Li, W. van Keulen, M. van de Ven, A. Molenaar, and G. Tang. Investigation on material properties and surface characteristics related to tyre-road noise for thin layer surfacings. Construction and Building Materials, 59:62 - 71, 2014.

[26] T. Li, R. Burdisso, and C. Sandu. Literature review of models on tire-pavement interaction noise. Journal of Sound and Vibration, 2018.

[27] G. Liao, M. S. Sakhaeifar, M. Heitzman, R. West, B. Waller, S. Wang, and Y. Ding. The effects of pavement surface characteristics on tire/pavement noise. Applied Acoustics, 76:14-23, 2014.

[28] B. Makwana, D. A. Bekke, B. de Bruijn, and SKP Amarnath. Modeling tools for the development of the safe and silent tyres. In EuroNoise 2015, pages 1025 - 1029, June 2015.

[29] M. Mokhtari. The effect of a tribo-modified surface layer on friction in elastomer contacts. PhD thesis, University of Twente, 2015. 
[30] F. G. Praticò, R. Fedele, and D. Vizzari. Significance and reliability of absorption spectra of quiet pavements. Construction and Building Materials, 140:274 - 281, 2017.

[31] FG Praticò and R Vaiana. A study on the relationship between mean texture depth and mean profile depth of asphalt pavements. Construction and Building Materials, 101:72-79, 2015.

[32] U. Sandberg. Road traffic noise - the influence of the road surface and its characterization. Appl. Acoust., 21(2):97-118, 1987.

[33] U. Sandberg and J. A. Ejsmont. Tyre/road noise reference book. 2002.

[34] A. F. Seybert and J. Han. Measurement of pavement absorption using ISO 13472-2. J. Acoust. Soc. Am., 123(5):3686, 2008.

[35] J. L. Vilaça, J. C. Fonseca, A. C. M. Pinho, and E. Freitas. 3d surface profile equipment for the characterization of the pavement texture-texscan. Mechatronics, 20(6):674-685, 2010.

[36] R. Wehr, A. Fuchs, and C. Aichinger. A combined approach for correcting tyre hardness and temperature influence on tyre/road noise. Applied Acoustics, 134(1):110-118, 2018. 



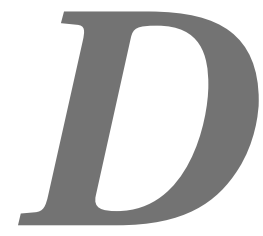

\section{Modelling approach for 3D printed sound absorbing panels}

This chapter is based on the paper 'Modelling approach for 3D printed sound absorbing panels', submitted to 'Acta Acustica united with Acustica' (2018). 


\title{
Modelling approach for 3D printed sound absorbing panels
}

\author{
M. Bezemer-Krijnen ${ }^{1)}$, Y.H. Wijnant ${ }^{1)}$, A. de Boer ${ }^{1)}$ \\ submitted to Acta Acustica united with Acustica (2018) \\ 1) Faculty of Engineering Technology, University of Twente, P.O. Box 217, 7500 AE Enschede, \\ The Netherlands. \\ m.bezemer@utwente.nl
}

\begin{abstract}
Sound absorbing panels are widely used for reducing noise and improving room acoustics. A new development is the use of additive manufacturing (AM) technologies to manufacture panels with complex shapes and at low costs. This paper discusses the sound absorbing properties of 3 D printed Polyamide 12 with the powder PA2200 as basis, manufactured with Selective Laser Sintering (SLS). The focus of this paper is on the additional sound reduction of the printed design caused by the sound absorption of the material itself. The impedance of the material is determined with measurements of thin slices of the printed material. A numerical modelling approach is developed which can be used to find the sound absorption of complex structures including the additional sound absorption by the material of the structure. Furthermore, the modelling approach includes both the scattering of the sound waves as well as the viscothermal effects inside the structure. This modelling approach is validated by impedance tube measurements of a complicated geometry of coupled tube resonators manufactured with SLS.
\end{abstract}




\section{Introduction}

Sound reducing panels are often used to improve room acoustics. To avoid production constraints, new additive manufacturing (AM) techniques, like Selective Laser Sintering (SLS), become increasingly popular. Using this $3 \mathrm{D}$ printing technique, custom designed, complex shapes can be produced, often at agreeable cost. The structures can be designed according to sound absorbing mechanisms and therefore, perform better than conventional absorbers. However, measurements show that the sound absorption of tube resonators manufactured with SLS and Polyamide 12, [12], does not agree well with the predicted sound absorption coefficient or with the sound absorption coefficient of the same tube resonators made from solid material. Therefore, the sound absorbing properties of the material itself has to be characterised before the acoustic properties of the sound reducing panels can be optimised.

\subsection{Background}

The sound absorption of structures, for locally reacting materials, could be predicted using impedance models, where the acoustic impedance $Z$ of a material is the ratio between the pressure $p$ and the particle velocity $v_{n}$ of a sound wave, in the direction normal to the surface of the material:

$$
Z=\frac{p}{v_{n}}
$$

There are several categories of impedance models. A category that is well-suited for structures of (coupled) resonators are microstructural models, in which the impedance is predicted using analytical relations based on the microstructure of the porous material or structure. The loss mechanisms causing sound absorption, like the viscous and thermal energy dissipation are analytically described using parameters as the effective density (viscous effects) and complex bulk modulus (thermal effects). To find the behaviour of the porous material, the properties of the individual pores are combined with general material properties, such as the tortuosity, flow resistivity and porosity as well as with the analytically found effective density and bulk modulus. Examples of these models are found in [1-3, 9].

The relation between the parameters of the microstructure and the bulk material are often based on Biot's theory, $[7,8]$. A distinction can be made between fluid-filled pores within a rigid frame or within an elastic frame. Since the geometry of the pores inside a porous material is often complex, a direct description of the pores can be difficult. In most models the viscous and thermal properties are estimated using the analytical relations for pores with a cylindrical cross-section or for slits, and are combined with one or more shape factors.

One of the models describing the sound pressure and particle velocity inside air-filled pores or tubes is the so-called low-reduced frequency (LRF) approach, in which the viscothermal properties of the air inside a prismatic tube are included in a complex wave propagation coefficient $\Gamma$. This LRF model is described in detail by $[4,10,13,14]$. The main relations are summarised in Appendix A. Advantages of the LRF model are that the pressure and particle velocity at any position inside the prismatic tube can be calculated analytically and that the LRF model is extensively validated for tube resonators and coupled structures of tube resonators, $[10,11$, 14].

\subsection{Objective}

The objective of this paper is to investigate if the sound absorption coefficient of 3D printed panels can be modelled including the sound absorption of the material itself. This paper focuses on a structure with tube resonators manufactured with SLS and the powder PA 2200, which results in Polyamide 12. Furthermore, the sound absorption and surface impedance for thin slices of this material is investigated using impedance tube measurements.

A numerical modelling approach where the properties of the air inside the tube resonators is described using analytical relations based on 
the LRF model is developed to predict the sound absorption coefficient for structures with sound absorbing material. This modelling approach is based on the analytical/numerical hybrid modelling approach, $[5,6]$. In the modelling approach used throughout this paper, the following aspects are included:

- the air inside the air-filled structure is modelled as viscous air;

- the scattering of the incident sound waves;

- the material of the structure is sound absorbing.

Furthermore, a structure with coupled resonators is designed to validate this modelling approach and to show the possibilities of these new AM techniques for broadband sound absorption in combination with this modelling approach.

\subsection{Outline}

In Section 2, the modelling approach, the influence of impedance boundary conditions on the sound absorption coefficient and the design of a structure with coupled tube resonators for broadband sound absorption are discussed.

Section 3, focuses on the measurements to determine the sound absorption of printed Polyamide 12, the acoustical performance of the designed structure and the validation of the numerical modelling approach.

\section{Modelling approach includ- ing sound absorption of mate- rial}

The sound absorption coefficient for sound reducing panels of (coupled) tube resonators can be found analytically using the LRF model, as described by [14] and summarized in Appendix B. In this approximation of the sound absorption coefficient, the sound absorption of the material itself and the coupling between the tubes is not included. Furthermore, the prediction of the sound absorption coefficient for more complex structures, like folded tubes, becomes less accurate.

\subsection{Modelling approach for normal inci- dent plane waves}

The modelling approach used in this paper is based on the hybrid modelling approach [5, $6]$, but adjusted to include the sound absorption of the material of the porous structure itself. Furthermore, since the models used in this paper are only solved for problems with normal incident plane waves inside an impedance tube, the modelling approach can be simplified such that the total sound field can be solved directly.

The structure of tube resonators is included in the numerical model. However, the properties of the air inside the tube resonators is determined analytically using the LRF model. The total sound field is solved for an incident sound pressure with unit amplitude.

Advantages of this hybrid modelling approach are:

- the viscothermal effects inside the resonators are included analytically, such acoustic boundary layers do not need to be modelled, which drastically reduces the mesh size and computational time;

- the viscous properties of the air inside the porous structure of tube resonators can be calculated and implemented for all tube resonators separately, since the sound field is solved numerically;

- the boundaries of the tube resonators can be changed from sound hard to sound absorbing by applying an impedance boundary condition;

- the inlet effects due to tube resonators in a baffle and the acoustic coupling between the resonators are automatically included by the numerical modelling approach. 


\subsection{Influence of impedance on sound absorption}

To include the sound absorption of the material in this hybrid modelling approach, the boundaries of the tube resonators are modelled with impedance boundary conditions. The influence of the applied impedance on the sound absorption coefficient is investigated. A simple model with one tube resonator is built, as shown in Figure 1a. The boundary conditions of this model are shown in Figure $1 b$.

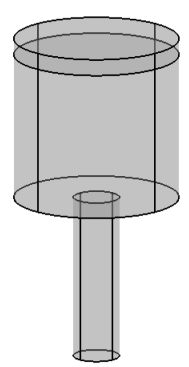

(a) Model with single tube resonator.

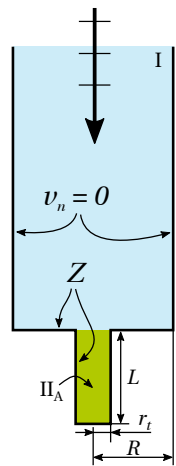

(b) Boundary conditions implemented in model.
Figure 1: Model with single tube resonator with length $L=50 \mathrm{~mm}$ and radius $r_{t}=7 \mathrm{~mm}$ inside an impedance tube with radius $R=25 \mathrm{~mm}$.

The sound absorption coefficient is defined as the ratio between the active sound intensity $\mathbf{I}_{\mathrm{ac}}$ and the incident sound intensity $I_{\text {in }}$, both integrated over a measurement surface $S$ :

$$
\alpha=\frac{\int \mathbf{I}_{\mathrm{ac}} \cdot \mathbf{n} d S}{\int I_{\mathrm{in}} d S}
$$

The active intensity is derived directly from the model:

$$
\mathbf{I}_{\mathrm{ac}}=\frac{1}{2} \operatorname{Re}(p \overline{\mathbf{v}})
$$

where $\overline{\mathbf{v}}$ denotes the complex conjugate of the complex particle velocity. The incident intensity can be described in terms of the complex amplitude $A$, assuming local plane waves, as described by [15].

$$
I_{\text {in }}=\frac{A \bar{A}}{2 \rho_{0} c_{0}}
$$

where $\bar{A}$ denotes the complex conjugate of $A$. The complex amplitude $A$ of the incident wave is defined by:

$$
A=\frac{1}{2}\left(p+\rho_{0} c_{0} v_{n}\right)
$$

where $p$ is the total sound pressure and $v_{n}$ the particle velocity in the direction normal to the measurement surface.

Multiple simulations are performed with different values for the impedance $Z$, according to:

$$
Z=a+b i
$$

The parameters $a$ and $b$ are varied between $-100 Z_{0}$ and $100 Z_{0}$, where $Z_{0}$ is the impedance of air. The parameters are varied such that the influence of the real part and the imaginary part of the impedance is investigated with respect to the impedance of air. The conclusions are summarised in Figure 2. The frequency of the peak in the sound absorption coefficient for $Z=100 Z_{0}$ is similar as for the model with for sound hard boundaries. The amplitude of the absorption peak is much larger compared to model with sound hard boundaries. These results show that the amplitude of the peak in the sound absorption coefficient depends on the real part of the impedance. The imaginary part of the impedance determines the frequency of the peak. When the imaginary part is positive, the peak shifts to higher frequencies and when negative, it shifts to lower frequencies.

It is noted that the results for an impedance with a negative real indicate sound emission. These results are therefore not shown in Figure 2 .

\subsection{Design and model of sample with coupled tube resonators}

To validate the adjusted hybrid modelling approach with impedance boundary conditions, 


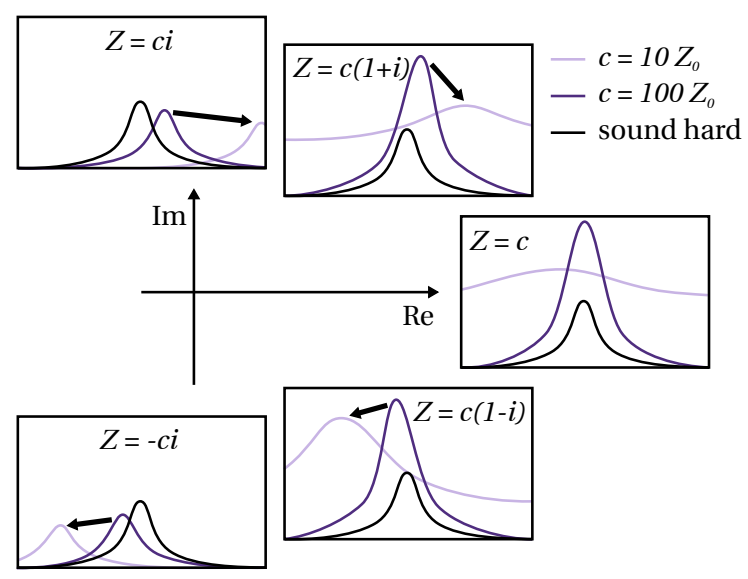

Figure 2: Influence of the impedance on the sound absorption coefficient.

a structure with coupled tube resonators for broadband sound absorption is designed. This structure consists of three coupled tube resonators and is manufactured with SLS and Polyamide 12 (with the powder PA 2200 as basis). A photo of the sample is shown in Figure 3.

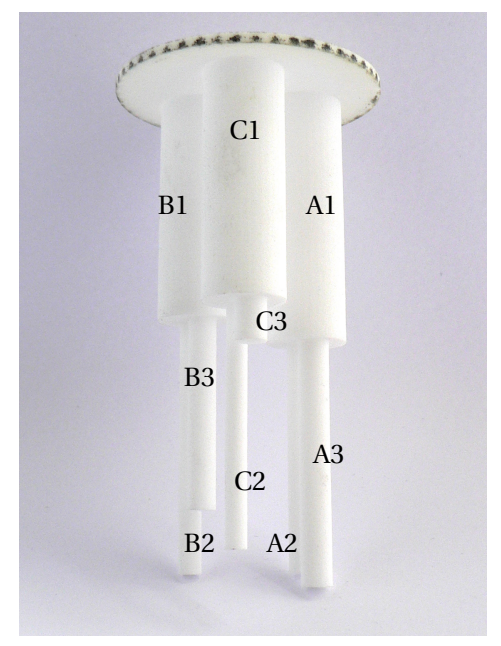

Figure 3: Designed sample with three coupled tube resonators. The numbering corresponds with the dimensions listed in Table 1.

The structure is designed for a high sound absorption between $f=1250 \mathrm{~Hz}$ and $f=2000 \mathrm{~Hz}$. The analytical solution for this sample is de- rived using the LRF theory for coupled tubes as described by [14] and summarised in Appendix B. The dimensions are listed in Table 1 .

\begin{tabular}{lcc}
\hline Tube & $\begin{array}{c}\text { Length } \\
{[\mathrm{mm}]}\end{array}$ & $\begin{array}{c}\text { Radius } \\
{[\mathrm{mm}]}\end{array}$ \\
\hline Tube A1 - top & 50.0 & 7.0 \\
Tube A2 - bottom & 47.5 & 1.5 \\
Tube A3 - bottom & 50.0 & 2.0 \\
\hline Tube B1 - top & 45.0 & 7.0 \\
Tube B2 - bottom & 52.5 & 1.25 \\
Tube B3 - bottom & 40.0 & 1.75 \\
\hline Tube C1 - top & 45.0 & 7.0 \\
Tube C2 - bottom & 47.5 & 1.0 \\
Tube C3 - bottom & 10.0 & 2.0 \\
\hline
\end{tabular}

Table 1: Dimensions of tube resonators. The wall thickness is $1 \mathrm{~mm}$.

A numerical model is made for the same geometry of coupled tube resonators, as shown in Figure $4 \mathrm{a}$. A schematic representation of the model and the implemented boundary conditions are shown in Figure 4b. Medium I represents the air inside an impedance tube, according to standard air conditions. Medium II represents the viscous air inside the tube resonators, determined using the LRF method for a tube with circular cross-section, as discussed in [10, 14]. The properties of the viscous air depend on the 
radius of the tubes and is different for all tube resonators. The total sound field is solved numerically for an incident wave of unit amplitude and the boundary conditions as shown in Figure $4 \mathrm{~b}$.

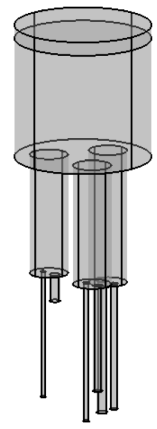

(a) Model with coupled tube resonators.

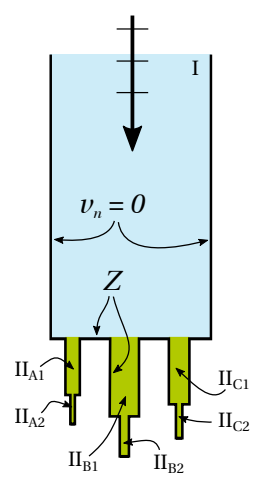

(b) Boundary conditions implemented in model.
Figure 4: Model of sample with coupled tube resonators inside an impedance tube with radius $R=$ $25 \mathrm{~mm}$.

\section{Validation of modelling approach}

The numerical modelling approach is validated for the 3D printed sample shown in Figure 3. The sound absorption coefficient is measured with the impedance tube technique, the method is discussed in Section 3.1. Section 3.2 describes the measurements to determine the sound absorption coefficient and surface impedance of the material of the sample, Polyamide 12 [12].

The validation of the numerical model including the sound absorption of the material itself is discussed in Section 3.3.

\subsection{Measurement method}

The impedance tube used for these measurements has 4 microphones at different locations. The dimensions of the impedance tube and the microphone locations are listed in Table 2 .

\begin{tabular}{lcc}
\hline Parameter & & Value \\
\hline Inner radius tube $[\mathrm{mm}]$ & $R$ & 25 \\
Outer radius tube $[\mathrm{mm}]$ & $R_{o}$ & 35 \\
Mic. distance 1-1 [mm] & $z_{1}$ & 0 \\
Mic. distance 1-2 [mm] & $z_{2}$ & 24 \\
Mic. distance 1-3 [mm] & $z_{3}$ & 62 \\
Mic. distance 1-4 [mm] & $z_{4}$ & 86 \\
Dist. mic. 1 to sample [mm] & $z_{s}$ & 280 \\
Cutoff frequency [Hz] & $f_{\text {co }}$ & $c_{0} /(4 R)$ \\
\hline
\end{tabular}

Table 2: Dimensions of impedance tube

For these measurements, the sound pressure level at all microphone positions are used to derive the complex amplitude of the incident waves $A$ and the reflected waves $B$. The measured sound pressure for a local plane wave (in longitudinal direction of the impedance tube) is given by:

$$
\tilde{p}(z, \omega)=A e^{-i k z_{i}}+B e^{i k z_{i}}
$$

where the microphone location can be denoted by $z_{i}$ and $k=\omega / c_{0}$ is the wave number, with $\omega$ is the angular frequency and $c_{0}$ is the speed of sound assuming standard air conditions. Substituting the microphone location for each microphone, the measured sound pressure can be written in matrix form:

$$
\left\{\begin{array}{c}
\tilde{p}_{1}\left(z_{1}, \omega\right) \\
\tilde{p}_{2}\left(z_{2}, \omega\right) \\
\vdots \\
\tilde{p}_{n}\left(z_{n}, \omega\right)
\end{array}\right\}=\left[\begin{array}{cc}
e^{-i k z_{1}} & e^{i k z_{1}} \\
e^{-i k z_{2}} & e^{i k z_{2}} \\
\vdots & \vdots \\
e^{-i k z_{n}} & e^{i k z_{n}}
\end{array}\right]\left\{\begin{array}{l}
A(\omega) \\
B(\omega)\}
\end{array}\right.
$$

This system of equations can be written as:

$$
\tilde{\mathbf{P}}=\mathbf{S a}
$$

The vector $\tilde{\mathbf{P}}$ contains the sound pressure measured by the microphones and the vector a contains the unknown complex amplitudes, $A(\omega)$ and $B(\omega)$. The matrix [S] describes the sound field in the impedance tube at the locations of the microphones. The system is solved using the normal equations, in a least square sense:

$$
\mathbf{a}=\left(\mathbf{S}^{T} \mathbf{S}\right)^{-1} \mathbf{S}^{T} \tilde{\mathbf{P}}
$$


where $\mathbf{S}^{T}$ is the conjugate transpose of S.

The ratio between the incident waves $p^{+}(\omega)=$ $A(\omega) e^{-i k z}$ and the reflected waves $p^{-}(\omega)=$ $B(\omega) e^{i k z}$ is called the reflection coefficient $R$ :

$$
R(\omega)=\frac{p^{-}}{p^{+}}=\frac{B(\omega)}{A(\omega)}
$$

The sound absorption coefficient $\alpha$ is then defined as:

$$
\alpha(\omega)=1-|R(\omega)|^{2}
$$

The impedance at the surface of the sample is derived using the complex amplitudes found with the measurements, combining Equations 1 and 10:

$$
Z_{s}(z, \omega)=\frac{A(\omega) e^{-i k z_{s}}+B(\omega) e^{i k z_{s}}}{\left(\rho_{0} c_{0}\right)^{-1}\left(A(\omega) e^{-i k z_{s}}-B(\omega) e^{i k z_{s}}\right)}
$$

where $z_{s}$ is the distance from the first microphone to the surface of the sample. The sound absorption coefficient based on this impedance is derived with Equation 12 using the reflection coefficient:

$$
R\left(z_{s}, \omega\right)=\frac{Z_{s}-Z_{0}}{Z_{s}+Z_{0}}
$$

where $Z_{0}=\rho_{0} c_{0}$, the impedance of air.

\subsection{Material properties}

To determine the sound absorption coefficient and the surface impedance of the material, thin slices of the printed material are measured inside an impedance tube. Samples with a thickness of $t=1 \mathrm{~mm}, t=2 \mathrm{~mm}, t=5 \mathrm{~mm}$ and $t=10 \mathrm{~mm}$ are tested, the samples are shown in Figure 5 .

\subsubsection{Sound absorption of material}

The slices are measured for two different situations:

- clamped inside a sample holder, such that there is an air column with height $H_{\text {air }}=100 \mathrm{~mm}$ behind the samples;

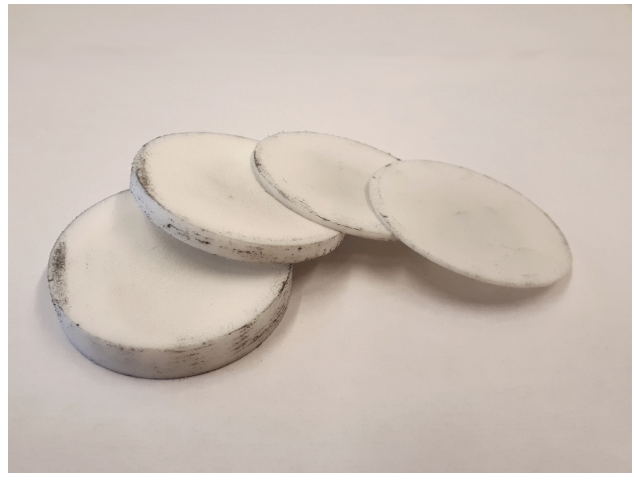

Figure 5: Slices of printed material with a thickness of $t=1 \mathrm{~mm}, t=2 \mathrm{~mm}, t=5 \mathrm{~mm}$ and $t=10 \mathrm{~mm}$.

- clamped against a sound hard backing of solid aluminum.

Figure 6 shows the sound absorption coefficient for the samples clamped such that there is a closed volume of air behind the sample. The results for the slices with a thickness of $t=1 \mathrm{~mm}$ and $t=2 \mathrm{~mm}$ show the influence of the structural resonances. For the sample with $t=1 \mathrm{~mm}$, the first resonance frequency is $f_{1}=1050 \mathrm{~Hz}$ and the second resonance frequency is $f_{2}=1765 \mathrm{~Hz}$. The slice with a thickness of $t=2 \mathrm{~mm}$, the resonance frequencies are: $f_{1}=1711 \mathrm{~Hz}$ and $f_{2}=1890 \mathrm{~Hz}$. Around the resonance frequencies, the sound absorption coefficient is low. The sound absorption coefficient for the sample with a thickness of $10 \mathrm{~mm}$ is somewhat larger than for the other three samples. However, the sound absorption coefficient for all samples is around $10 \%$.

The sound absorption coefficient for the printed samples with sound hard backing is shown in Figure 7. Again, the sample with $t=10 \mathrm{~mm}$ has a sound absorption coefficient which is larger than for the other three samples. However, the sample with a thickness of $1 \mathrm{~mm}$ has a larger sound absorption coefficient than expected based on the thickness and the results shown in Figure 6. It is assumed this additional sound absorption is introduced by the measurement uncertainties. For example, it could be that the sample is not clamped tightly against the backing, such that a small (viscous) air layer between the sample and the backing 


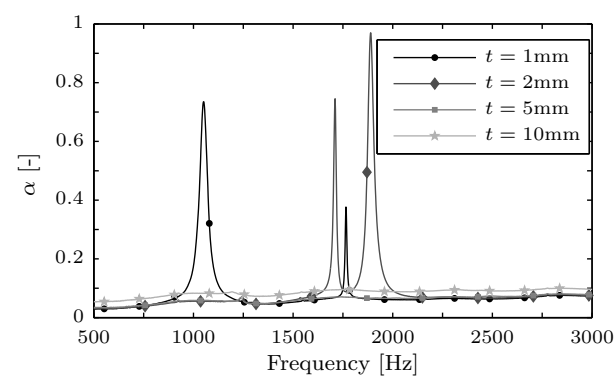

Figure 6: Sound absorption coefficient of the printed samples inside the sample holder including a volume of air behind the sample.

exists.

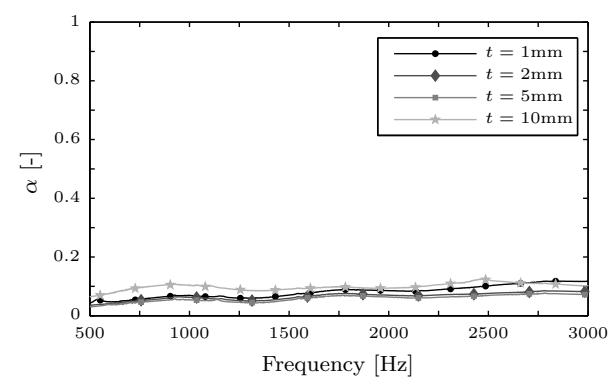

Figure 7: Sound absorption coefficient of printed samples pressed against sound hard backing.

\subsubsection{Impedance of material}

The impedance of the sample with a thickness of $t=1 \mathrm{~mm}$ is shown in Figure 8. The surface impedance is found with Equation 13 and depends on the frequency. To implement this impedance in the numerical model, a polynomial fit based on the measurements is derived:

$$
\begin{aligned}
Z_{s, 1 \mathrm{~mm}}= & \left(0.00250 f^{2}-13.49 f+18980\right)+ \\
& \left(0.00179 f^{2}-11.03 f+20610\right) i
\end{aligned}
$$

where $f=\omega / 2 \pi$ is the frequency. The polynomial fit is shown in Figure 8. The measurements accuracy is low below $f=600 \mathrm{~Hz}$, these frequencies are therefore not included in the polynomial fit. In addition, the frequency range of interest for the validation of the modelling approach is between $f=1250 \mathrm{~Hz}$ and $f=$ $2000 \mathrm{~Hz}$.
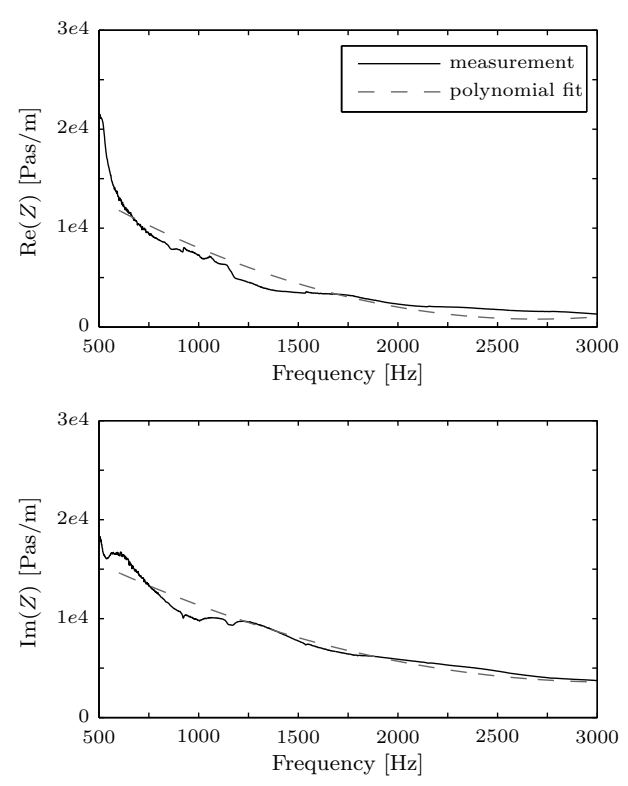

Figure 8: Real and imaginary part of the surface impedance of the slice with $t=1 \mathrm{~mm}$. The polynomial fit $Z_{s, 1 \mathrm{~mm}}=\left(0.00250 f^{2}-13.49 f+18980\right)+$ $\left(0.00179 f^{2}-11.03 f+20610\right) i$ of the measured impedance is indicated with the dashed line.

This polynomial function of surface impedance for the slice with $t=1 \mathrm{~mm}$ is implemented in a simple model of a sample placed inside an impedance tube with a radius $R=25 \mathrm{~mm}$, as is schematically shown in Figure 9. The predicted sound absorption coefficient by this model is shown in Figure 10, together with the measured sound absorption coefficient and the sound absorption coefficient based on the polynomial fit of the impedance $Z_{s, 1 \mathrm{~mm}}$. The sound absorption coefficient found by substituting $Z_{s, 1 \mathrm{~mm}}$ in Equation 14 and the sound absorption coefficient predicted with the numerical model correspond well with the measured sound absorption coefficient. 


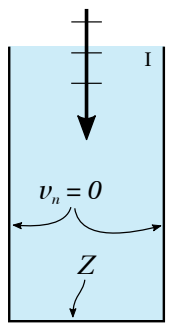

Figure 9: Boundary conditions for the numerical model of sample within impedance tube, where the sample is modelled with an impedance boundary condition.

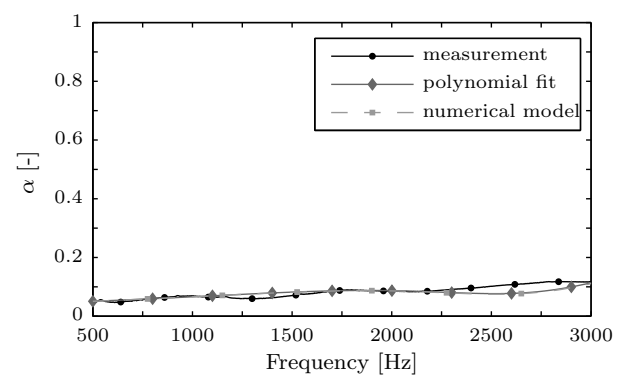

Figure 10: Sound absorption coefficient for slice with thickness $t=1 \mathrm{~mm}$ : measured, derived from fit of impedance $Z_{s, 1 \mathrm{~mm}}$ and predicted with model using $Z_{s, 1 \mathrm{~mm}}$ for the impedance boundary condition.

\subsection{Validation for structure with cou- pled tube resonators}

The sample described in Section 2.3 is manufactured using SLS with Polyamide 12. The wall thickness of the sample is $t=1 \mathrm{~mm}$. After the printing process, the small tube resonators have been cleaned manually using a thin pin. During the measurements, the sample is clamped inside a sample holder and placed in the impedance tube. The tube is closed at the end opposite the speaker, such that the air behind the sample forms a closed volume.

The measured sound absorption coefficient is compared to the sound absorption coefficient estimated with the analytical relations using the LRF model (Appendix B) and the sound absorption coefficient predicted by the numerical model with sound hard boundaries. This

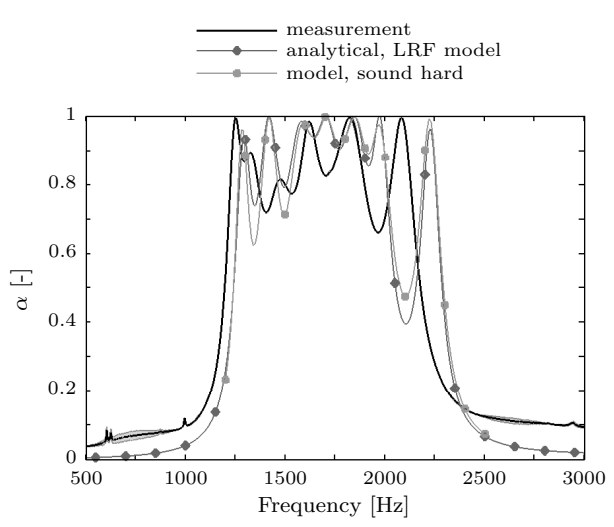

Figure 11: Measured sound absorption coefficient of the sample with coupled tube resonators, the shaded area indicates the \pm 1 standard deviation. The sound absorption coefficient based on the LRF model and the sound absorption coefficient predicted with the numerical modelling approach for sound hard boundaries are also included in this figure.

is shown in Figure 11. The analytical relations for the sound absorption coefficient of systems with coupled tubes have been validated by [14] for samples made from sound hard materials. The sound absorption coefficient predicted by the numerical model with sound hard boundaries corresponds very well with the analytical solution.

The measurement results of the sample show that the peaks in the sound absorption coefficient have shifted to lower frequencies. Therefore, even if the sound absorption coefficient of the material itself is around $10 \%$, it has influence on the sound absorption of the structure with coupled resonators. It is expected that only the surfaces perpendicular to longitudinal direction of the impedance tube contribute to this additional sound absorption, since plane wave propagation is assumed.

According to conclusions shown in Figure 2, an impedance of the shape $Z=a-b i$ is expected. However, the measurements of the slices show that the impedance of the material has the shape $Z=a+b i$. 
The polynomial fit of the surface impedance of the sample with a thickness of $t=1 \mathrm{~mm}$, $Z_{s, 1 \mathrm{~mm}}$ in Equation 15, is used for the impedance boundary conditions in the hybrid model. The resulting sound absorption coefficient is shown in Figure 12. However, this sound absorption coefficient does not correspond with the measured sound absorption coefficient. The peaks in the sound absorption coefficient have mostly disappeared, suggesting too much sound absorption inside the tube resonators. In Figure 2 is shown that this behaviour indicates that the imaginary part of the impedance applied at the boundaries in the numerical model is too low.

Based on these results, a small study is performed to derive an impedance which will give a sound absorption coefficient similar to the measurement results, using the shape factors $\tau_{\mathrm{Re}}$ and $\tau_{\mathrm{Im}}$ :

$$
\begin{aligned}
Z_{\text {model }}= & \tau_{\operatorname{Re}}\left(0.00250 f^{2}-13.49 f+18980\right)+ \\
& \tau_{\operatorname{Im}}\left(0.00179 f^{2}-11.03 f+20610\right) i
\end{aligned}
$$

Since an impedance with the shape $Z=a-b i$ was expected, the sign of the shape factor for the imaginary part was changed. Furthermore, for a larger negative value of $\tau_{\mathrm{Im}}$, the peak behaviour seen in the measured sound absorption coefficient was also predicted by the hybrid model. The shape factor $\tau_{\mathrm{Re}}$ was set to 1 , since increasing the real part of the impedance mostly affects the amplitude of the peaks in the sound absorption coefficient. For example, when using $\tau_{\mathrm{Re}}=$ 15 the amplitude of the peaks is larger and the difference in amplitude between the different peaks is smaller.

Figure 12 shows the predicted sound absorption coefficient for the impedance $Z_{\text {model }}$ with the shape factors $\tau_{\mathrm{Re}}=1$ and $\tau_{\mathrm{Im}}=-15$. This predicted sound absorption coefficient corresponds fairly well with the measured sound absorption coefficient. Therefore, it is concluded that the developed modelling approach can be used to predict the sound absorption coefficient of a structure of coupled tube resonators, including the sound absorption of the material itself. However, more research should be done to obtain a better understanding of these shape

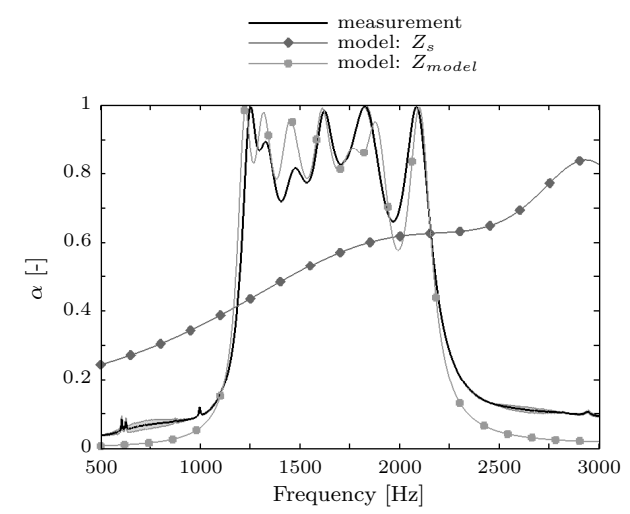

Figure 12: Measured sound absorption coefficient of the sample with coupled tube resonators compared to the predicted sound absorption coefficient with the numerical modelling approach including the sound absorption of the material.

factors, since the impedance used in the hybrid modelling approach is based on measured surface impedance for a slice of the same material.

\section{Conclusions and recommenda- tions}

The main goal of this research was to predict the sound absorption coefficient of 3D printed sound reducing panels. This paper is focused on the material Polyamide 12 and the Selective Laser Sintering (SLS) technique. SLS techniques are increasingly popular when designing and manufacturing sound reducing panels due to the design freedom and quick and low-cost production.

The measurements in this paper shows that even though the sound absorption coefficient of the material is low, it influences the sound absorption of a structure manufactured with this material. A hybrid modelling approach is developed to predict the sound absorption coefficient of a sound reducing structure or panel for normal incident plane waves. The additional 
sound absorbing properties are included in this modelling approach by impedance boundary conditions. Furthermore, this modelling approach includes the viscothermal effects inside the air-filled structure and the scattering of the sound waves at the surface of the structure.

The sound absorption coefficient and surface impedance of Polyamide 12 are determined with the impedance tube technique for thin slices of this material. A frequency dependent polynomial fit of the measured impedance is implemented in the hybrid model. The modelling approach is validated for a structure of coupled tube resonators, designed for broadband sound absorption. The results show that the developed model of this structure can predict the sound absorption coefficient of complicated systems of tube resonators. Furthermore, the shift in the frequency of the peaks in the sound absorption caused by the additional sound absorbing properties of the material can be predicted by the model as well. However, more research is needed to obtain a better understanding as to why the measured surface impedance does not give the expected results when used in the hybrid modelling approach.

Nonetheless, the example of the structure with coupled tube resonators shows the potential for broadband sound absorption using these new techniques. The obtained sound absorption coefficient is large within the frequency range of the optimisation. Furthermore, the manufacturing techniques give a large design freedom, which makes is possible to design structures with for example folded tubes which will reduce the thickness of the sound reducing panels.

\section{Acknowledgements}

We thank Foteini Setaki for involving us in her research about designing compact sized sound reducing panels manufactured with SLS for broadband sound absorption.

\section{Appendix A: Low-reduced frequency model}

Below, the most important relations and properties of the low-reduced frequency (LRF) model are given. More information can be found in $[4,10,13,14]$.

The sound pressure $p(x, \omega)$ and particle velocity $v(x, \omega)$ inside this prismatic tube is given by:

$$
\begin{array}{r}
p(x, \omega)=A e^{\Gamma(\omega) k(\omega) x}+B e^{-\Gamma(\omega) k(\omega) x} \\
v(x, \omega)=\frac{G(\omega)}{\rho_{0} c_{0}}\left[A e^{\Gamma(\omega) k(\omega) x}-B e^{-\Gamma(\omega) k(\omega) x}\right]
\end{array}
$$

where $A$ and $B$ are the complex amplitudes of the incident and reflected waves, respectively, and $k(\omega)=c_{0} / \omega$ is the wave number with $\omega$ is the angular velocity and $c_{0}$ is the speed of sound assuming standard air conditions. The coefficient $G(\omega)$ depends on the geometry of the cross-section of the prismatic tube. The sound absorbing loss mechanisms are included in the viscothermal wave propagation coefficient $\Gamma$ :

$$
\Gamma(\omega)=\sqrt{\frac{J_{0}(i \sqrt{i} s(\omega))}{J_{2}(i \sqrt{i} s(\omega))} \frac{\gamma}{n(\omega)}}
$$

where $J_{0}$ and $J_{2}$ are the Bessels functions of the first kind of the order 0 and 2, $s$ is the shear wave number, $\gamma$ ratio of specific heats at constant pressure $C_{p}$ and constant volume $C_{V}, N_{\mathrm{Pr}}$ is the Prandtl number and $n$ is the polytropic coefficient:

$$
\begin{aligned}
s(\omega) & =r_{t} \sqrt{\frac{\rho_{0} \omega}{\mu}} \\
\gamma & =\frac{C_{p}}{C_{V}} \\
N_{\mathrm{Pr}} & =\frac{\mu C_{p}}{\lambda} \\
n(\omega) & =\left[1+\frac{\gamma-1}{\gamma} \frac{J_{2}\left(i \sqrt{i N_{\mathrm{Pr}}} s(\omega)\right)}{J_{0}\left(i \sqrt{i N_{\mathrm{Pr}}} s(\omega)\right)}\right]^{-1}
\end{aligned}
$$

where $\mu$ is the dynamic viscosity and $\lambda$ is the thermal conductivity. For a tube with a circular cross-section, the coefficient $G(\omega)$ yields:

$$
G(\omega)=-\frac{i}{\Gamma(\omega)} \frac{\gamma}{n(\omega)}
$$




\section{Appendix B: Analytical relations for coupled tube resonators}

For a panel with multiple tubes with different dimensions, the impedance of the panel is given by the summation of the contributions of the surface impedance $Z_{j}$ of the individual tubes:

$$
Z_{\text {panel }}=\frac{1}{\sum_{j=1}^{N} \frac{\Omega_{s, j}}{Z_{j}}}
$$

where $N$ is the amount of tubes in the panel, $\Omega_{s, j}$ is the surface porosity of the $j$ th tube and $Z_{j}$ is the surface impedance of the $j$ th tube.

For a tube resonator with length $L$ and with one open end at $z=0$ and one closed end at $z=L$, the surface impedance is found with:

$$
Z_{j}(z=0)=-\frac{\rho_{0} c_{0}}{G_{j}} \frac{\sinh (\Gamma k L)}{\cosh (\Gamma k L)-\frac{p_{1}}{p_{0}}}
$$

where $p_{0}$ is the pressure at $z=0$ and $p_{1}$ is the pressure at $z=L$ and the transfer function for these boundary conditions yields:

$$
\frac{p_{1}}{p_{0}}=\frac{1}{\cosh (\Gamma k L)}
$$

The transfer function for the pressure at the opening of two tubes coupled in series, with the pressure $p_{j-1}$ at the one open end of the first tube (tube $J$ ) and $p_{j}$ at the end coupled with a second tube (tube $J+1$ ) is, [14]:

$$
\begin{aligned}
\frac{p_{j-1}}{p_{j}}= & {\left[\cosh (\Gamma k L)_{J}+\frac{\sinh (\Gamma k L)_{J}}{S_{J} G_{J}}\right.} \\
& \left.\left(\frac{S_{J+1} G_{J+1}}{\sinh (\Gamma k L)_{J+1}}\left\{\cosh (\Gamma k L)_{J+1}-\frac{p_{j+1}}{p_{j}}\right\}\right)\right]^{-1}
\end{aligned}
$$

where $\frac{p_{j+1}}{p_{j}}$ is the transfer function of the pressure of tube $J=1$. For a system of coupled resonators with two tubes in parallel attached to a third tube in series, the transfer function $\frac{p_{j-1}}{p_{j}}$ is found with:

$$
\begin{aligned}
\frac{p_{j-1}}{p_{j}}= & {\left[\cosh (\Gamma k L)_{J}+\frac{\sinh (\Gamma k L)_{J}}{S_{J} G_{J}}\right.} \\
& \left(\frac{S_{J+1} G_{J+1}}{\sinh (\Gamma k L)_{J+1}}\left\{\cosh (\Gamma k L)_{J+1}-\frac{p_{j+1}}{p_{j}}\right\}\right. \\
& \left.\left.+\frac{S_{J+2} G_{J+2}}{\sinh (\Gamma k L)_{J+2}}\left\{\cosh (\Gamma k L)_{J+2}-\frac{p_{j+2}}{p_{j}}\right\}\right)\right]^{-1}
\end{aligned}
$$

where tube $J+2$ is added at the end of tube $J$, such that tube $J+1$ and tube $J+2$ are coupled in parallel.

\section{References}

[1] J. Allard and N. Atalla. Propagation of Sound in Porous Media: Modelling Sound Absorbing Materials 2e. John Wiley \& Sons, 2009.

[2] K. Attenborough. Ground parameter information for propagation modeling. $J$. Acoust. Soc. Am., 92:418-427, 1992.

[3] K. Attenborough, K. M. Li, and K. Horoshenkov. Predicting outdoor sound. Taylor \& Francis, 2007.

[4] W. M. Beltman. Viscothermal wave propagation including acousto-elastic interaction. PhD thesis, University of Twente, 1998.

[5] M. Bezemer-Krijnen, Y. H. Wijnant, and A. de Boer. Predicting sound absorption of stacked spheres: combining an analytical and numerical approach. Acta Acustica united with Acustica, 102:971-983, aug 2016.

[6] M. Bezemer-Krijnen, Y. H. Wijnant, and A. de Boer. Prediction of sound absorption of stacked granular materials for oblique incident sound waves. Acta Acustica united with Acustica, 2017. submitted.

[7] M. A. Biot. Theory of propagation of elastic waves in a fluid-saturated porous solid. i. low-frequency range. Acoustical Society of America, 28(2):168-178, march 1956. 
[8] M. A. Biot. Theory of propagation of elastic waves in a fluid-saturated porous solid. ii. higher frequency range. Acoustical Society of America, 28(2):179-191, march 1956.

[9] Y. Champoux and M. R. Stinson. On acoustical models for sound propagation in rigid frame porous materials and the influence of shape factors. J. Acoust. Soc. Am., 92:1120-1131, 1992.

[10] M. H. C. Hannink. Acoustic resonators for the reduction of sound radiation and transmission. PhD thesis, University of Twente, 2007.

[11] M. H. C. Hannink., Y. H. Wijnant, and A. de Boer. Optimised sound absorbing trim panels for the reduction of aircraft cabin noise. In Eleventh International Congress on Sound and Vibration, ICVS 11, pages 1855-1862, 2004.

[12] EOS GmbH Electro Optical Systems. Pa 2200 / pa 12.

[13] H. Tijdeman. On the propagation of sound waves in cylindrical tubes. J. Sound Vib., 39(1):1-33, 1975.

[14] F. J. M. van der Eerden. Noise reduction with coupled prismatic tubes. $\mathrm{PhD}$ thesis, University of Twente, 2000.

[15] Y. H Wijnant, E. R. Kuipers, and A. de Boer. Development and application of a new method for the insitu measurement of sound absorption. In Proceeding of ISMA2010 including USD2010, pages 109122, 2010. 



\section{Part III}

Appendices 



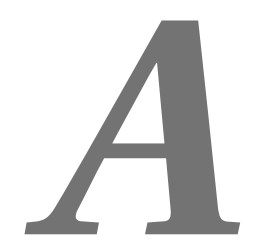

\section{Properties of air}

The properties of air as used and referred to throughout this thesis.

\begin{tabular}{llll}
\hline Property & Symbol & Value & Unit \\
\hline density & $\rho_{0}$ & 1.2041 & {$\left[\mathrm{kgm}^{-3}\right]$} \\
speed of sound & $c_{0}$ & 343.21 & {$\left[\mathrm{~ms}^{-1}\right]$} \\
reference sound pressure & $P_{0}$ & $20 \cdot 10^{-6}$ & {$[\mathrm{~Pa}]$} \\
dynamic viscosity & $\mu$ & $18.27 \cdot 10^{-6}$ & {$[\mathrm{Pas}]$} \\
shear velocity & $\eta$ & $18.4 \cdot 10^{-6}$ & {$\left[\mathrm{kgm}^{-1} \mathrm{~s}^{-1}\right]$} \\
Prandtl number & $N_{\operatorname{Pr}}$ & 0.71 & {$[-]$} \\
specific heat ratio & $\gamma$ & 1.4 & {$[-]$} \\
\hline
\end{tabular}

Table A.1: Properties of air 



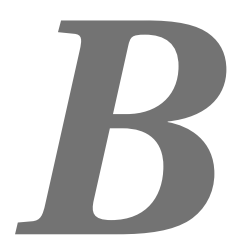

\section{Tube resonators}

\section{Introduction}

Using the LRF modelling approach, the sound pressure and particle velocity, and thus the impedance, inside a tube with a prismatic cross-section can be derived analytically. The theory can be expanded to find the surface impedance and sound absorption coefficient of arrays and systems of (coupled) tube resonators. In this appendix the impedance and sound absorption coefficient for a single tube resonator is given, as well as the relations for tube resonators in parallel and in series. All the relations described in this appendix are for resonators with a circular cross-section. These relations and more information about the derivation of these relations are found in [56] and [31].

\section{Sound absorption coefficient for tube resonators}

The sound absorption coefficient $\alpha$ is given by:

$$
\alpha=1-|R|^{2}
$$

with the reflection coefficient $R$ :

$$
R=\frac{Z_{\text {panel }}-\rho_{0} c_{0}}{Z_{\text {panel }}+\rho_{0} c_{0}}
$$

where $\rho_{0}$ is the density of air, $c_{0}$ is the speed of sound in air and $Z_{\text {panel }}$ is the impedance of a panel of resonators with a total surface area of $S_{\text {panel }}$ and a surface porosity of $\Omega_{s}$. The surface porosity of a wall with $N$ identical tubes is defined as:

$$
\Omega_{s}=\frac{N S_{J}}{S_{\text {panel }}}
$$

where $S_{J}=\pi r_{t}^{2}$ is the area of the tube resonator $J$ with radius $r_{t}$. 
Assuming a small control volume above the panel with resonators with conservation of mass and plane waves, the surface impedance of the panel is derived [56]:

$$
Z_{\text {panel }}=\frac{Z_{J}}{\Omega_{s}}
$$

where $Z_{J}$ is the surface impedance of a single tube.

\subsection{Single tube resonator}

The surface impedance of the cylindrical tube $J$ with one closed end and one open end and length $L_{J}$ is defined by:

$$
Z_{J}=-\frac{\rho_{0} c_{0}}{G_{J}} \tanh ^{-1}(\Gamma k L)_{J}
$$

Due to inlet effects at the entrance of the tube, the effective length is slightly larger than the physical length of the tube resonator. This is corrected by adding an end correction $\delta_{J}$ to the length of the tube. The end correction depends on the configuration of the tube in the panel. For a single tube inside an infinite baffle the correction yields:

$$
\delta_{J}=\frac{8 r_{J}}{3 \pi}
$$

For a single tube positioned at the centre of a larger tube with a circular crosssection with radius $r_{o}$ is given by:

$$
\delta_{J}=\frac{8 r_{J}}{3 \pi}\left[1-1.25 \frac{r_{J}}{r_{o}}\right]
$$

with $r_{J} / r_{o}<0.6$.

\subsection{Tube resonators in parallel}

For a panel with multiple tubes with different dimensions, the impedance of the panel is given by the summation of the contributions of the surface impedance $Z_{J}$ of the individual tubes:

$$
Z_{\text {panel }}=\frac{1}{\sum_{J=1}^{N} \frac{\Omega_{s, J}}{Z_{J}}}
$$

where $N$ is the amount of tubes in the panel.

The end correction for a resonator in a panel with equally spaced resonators with distance $a$, the end correction yields:

$$
\delta_{J}=\frac{8 r_{J}}{3 \pi}\left[1-0.44 \frac{r_{J}}{a}\right]
$$

with $a<2 r_{J}$. 


\subsection{Tube resonators in series}

The impedance of a tube can be expressed as the dimensionless impedance $\zeta$, [56]:

$$
\zeta=-\frac{G_{J}}{\rho_{0} c_{0}} Z
$$

For a tube with one open end at $z=0$ and one closed end at $z=L_{J}$ the impedance at $z=0$ is found with:

$$
\zeta(0)=\frac{\sinh (\Gamma k L)_{J}}{\cosh (\Gamma k L)_{J}-\frac{p_{1}}{p_{0}}}
$$

where $p_{0}$ is the pressure at $z=0$ and $p_{1}$ is the pressure at $z=L_{J}$ and the transfer function for these boundary conditions yields:

$$
\frac{p_{1}}{p_{0}}=\frac{1}{\cosh (\Gamma k L)}
$$

Combining Equations B.10, B.11 and B.12 results in the surface impedance for a single tube resonator (Equation B.5).

The transfer function for the pressure at the opening of two tubes coupled in series, with the pressure $p_{j-1}$ at the one open end of the first tube (tube $J$ ) and $p_{j}$ at the end coupled with a second tube (tube $J+1$ ) is, [56]:

$$
\frac{p_{j-1}}{p_{j}}=\left[\cosh (\Gamma k L)_{J}+\frac{\sinh (\Gamma k L)_{J}}{S_{J} G_{J}}\left(\frac{S_{J+1} G_{J+1}}{\sinh (\Gamma k L)_{J+1}}\left\{\cosh (\Gamma k L)_{J+1}-\frac{p_{j+1}}{p_{j}}\right\}\right)\right]^{-1}
$$

where $p_{j+1} / p_{j}$ is the transfer function of the pressure of tube $J=1$. When a third tube (tube $J+2$ ) is added at the end of tube $J$, such that tube $J+1$ and tube $J+2$ are coupled in parallel, the transfer function $p_{j-1} / p_{j}$ yields:

$$
\begin{aligned}
\frac{p_{j-1}}{p_{j}}= & {\left[\cosh (\Gamma k L)_{J}+\frac{\sinh (\Gamma k L)_{J}}{S_{J} G_{J}}\left(\frac{S_{J+1} G_{J+1}}{\sinh (\Gamma k L)_{J+1}}\left\{\cosh (\Gamma k L)_{J+1}-\frac{p_{j+1}}{p_{j}}\right\}\right.\right.} \\
& \left.\left.+\frac{S_{J+2} G_{J+2}}{\sinh (\Gamma k L)_{J+2}}\left\{\cosh (\Gamma k L)_{J+2}-\frac{p_{j+2}}{p_{j}}\right\}\right)\right]^{-1}
\end{aligned}
$$

\section{Aluminum sample with 37 parallel tube resonators}

A sample with 37 parallel tube resonators is designed to validate the hybrid modelling approach for one-dimensional plane waves, as described in Section 2.2. The most important design restraints were:

- outer diameter of $D=50 \mathrm{~mm}$, such that the sample can be tested in the available impedance tube;

- maximum length of resonators is $L=100 \mathrm{~mm}$; 
- equal radius for all resonators;

- broadband sound absorption.

The frequency range for the optimisation is based on the maximum length of the resonator: the first resonance frequency $f_{1}=c_{0} /(4 L)=857.5 \mathrm{~Hz}$ has been used as lower frequency limit. To obtain broadband sound absorption, the second resonance frequency $f_{2}=3 c_{0} /(4 L)=2572.5 \mathrm{~Hz}$ is used as the upper limit of the frequency range, such that the higher resonance frequencies contribute to the sound absorption coefficient as well. Therefore, the frequency range for the optimisation is set to $850 \mathrm{~Hz}-2500 \mathrm{~Hz}$.

The radius of the tube resonators is based on the optimal surface porosity, derived from Equation B.3. The maximum number of resonators is determined based on the outer diameter and the manufacturing constraints.

A simple optimisation algorithm is used to maximise the sound absorption coefficient where the length of each resonator is the only degree of freedom [14, 31]. The sound absorption coefficient is determined by combining the surface impedance of all tube resonator (according to Equation B.5), with the length correction for a single tube inside a larger tube (Equation B.7).

The optimisation resulted in a structure of 37 parallel tube resonators with a radius of $r_{t}=3 \mathrm{~mm}$. A photo of the aluminium sample is shown in Figure B.1a. The dimensions and coordinates of the tubes are listed in Table B.1, using the numbering in Figure B.1b.

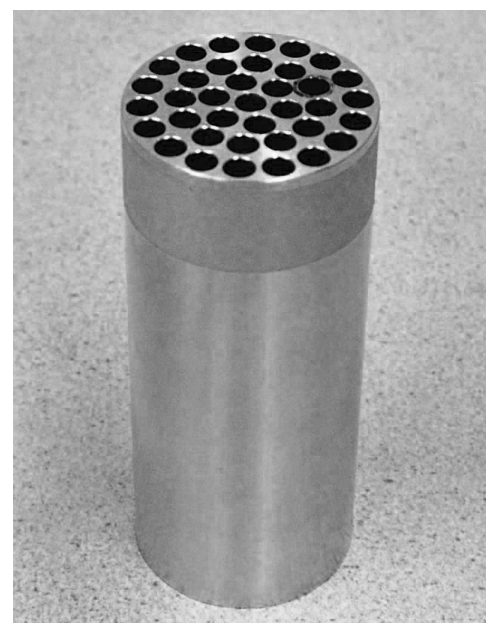

(a) Photo of sample with 37 tubes.

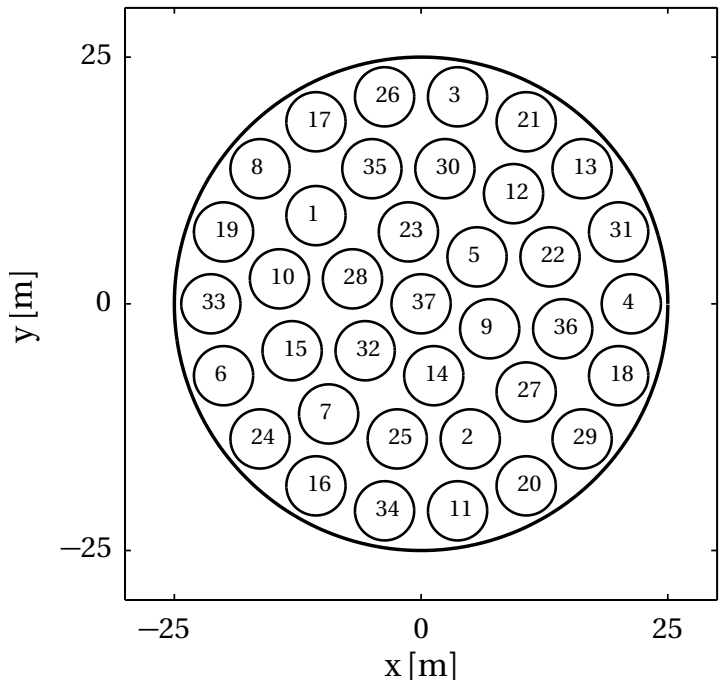

(b) Design of sample with 37 tubes.

Figure B.1: Aluminium sample with 37 tubes. 


\begin{tabular}{rrrr|rrrr}
$\mathrm{N}$ & $\begin{array}{r}L_{\text {design }} \\
{[\mathrm{mm}]}\end{array}$ & $\begin{array}{r}\mathrm{x} \\
{[\mathrm{mm}]}\end{array}$ & $\begin{array}{c}\mathrm{y} \\
{[\mathrm{mm}]}\end{array}$ & $\mathrm{N}$ & $\begin{array}{r}L_{\text {design }} \\
{[\mathrm{mm}]}\end{array}$ & $\begin{array}{c}\mathrm{x} \\
{[\mathrm{mm}]}\end{array}$ & $\begin{array}{c}\mathrm{y} \\
{[\mathrm{mm}]}\end{array}$ \\
\hline 1 & 100.0 & -10.4 & 8.8 & 21 & 55.3 & 10.4 & 18.1 \\
2 & 100.0 & 4.9 & -13.4 & 22 & 53.3 & 12.8 & 4.7 \\
3 & 99.0 & 3.6 & 20.6 & 23 & 51.4 & -1.3 & 7.1 \\
4 & 98.0 & 20.9 & 0.0 & 24 & 49.6 & -16.0 & -13.4 \\
5 & 95.6 & 5.6 & 4.7 & 25 & 47.8 & -2.4 & -13.4 \\
6 & 92.8 & -19.6 & -7.1 & 26 & 46.1 & -3.6 & 20.6 \\
7 & 90.1 & -9.2 & -10.9 & 27 & 44.5 & 10.4 & -8.8 \\
8 & 87.3 & -16.0 & 13.4 & 28 & 42.9 & -6.8 & 2.5 \\
9 & 84.6 & 6.8 & -2.5 & 29 & 41.4 & 16.0 & -13.4 \\
10 & 81.8 & -14.1 & 2.5 & 30 & 40.0 & 2.4 & 13.4 \\
11 & 79.1 & 3.6 & -20.6 & 31 & 38.6 & 19.6 & 7.1 \\
12 & 76.4 & 9.2 & 10.9 & 32 & 37.3 & -5.6 & -4.7 \\
13 & 73.8 & 16.0 & 13.4 & 33 & 36.0 & -20.9 & 0.0 \\
14 & 71.3 & 1.3 & -7.1 & 34 & 34.8 & -3.6 & -20.6 \\
15 & 68.8 & -12.8 & -4.7 & 35 & 33.7 & -4.9 & 13.4 \\
16 & 66.4 & -10.4 & -18.1 & 36 & 32.6 & 14.1 & -2.5 \\
17 & 64.0 & -10.4 & 18.1 & 37 & 31.0 & 0.0 & 0.0 \\
18 & 61.7 & 19.6 & -7.1 & & & & \\
19 & 59.5 & -19.6 & 7.1 & & & & \\
20 & 57.4 & 10.4 & -18.1 & & & &
\end{tabular}

Table B.1: Dimensions and coordinates of sample with 37 parallel tubes, according to design.

\section{Printed sample with coupled tube resonators}

A sample with three coupled tube resonators is designed to research the sound absorbing properties of polyamide (PA12), a material used in combination with the SLS technique, as described in Section 2.5. The most important design restraints were:

- outer diameter of $D=50 \mathrm{~mm}$, such that the sample can be tested in the available impedance tube;

- three resonators, each connected in series to two tube resonators in parallel;

- maximum length of coupled resonators $L=100 \mathrm{~mm}$;

- thin-walled design;

- tube radius within manufacturing constraints;

- broadband sound absorption.

The maximum length of the sample has been used to find the lower limit of the frequency range used for the optimisation. The upper limit was chosen such that 
the averaged sound absorption coefficient within the frequency range was above $\alpha=0.9$, while having three coupled resonators.

The radius of the first level of tube resonators is mostly based on the outer radius and the wall thickness of the sample. For all three resonators, this radius was set to $r_{t}=7 \mathrm{~mm}$. Again, a simple optimisation algorithm is used to optimise the sound absorption coefficient within the set frequency range. Both the length and the radius of the tubes were variables in the optimisation procedure. However, to limit the number of degrees of freedom, the radius of the first level of tubes was fixed. The sound absorption coefficient is determined by combining the relations in Equation B.11, B.12, B.13 and B.14.

A photo of the sample with the coupled resonators is shown in Figure B.2. The dimensions and coordinates of the tubes are listed in Table B.2.

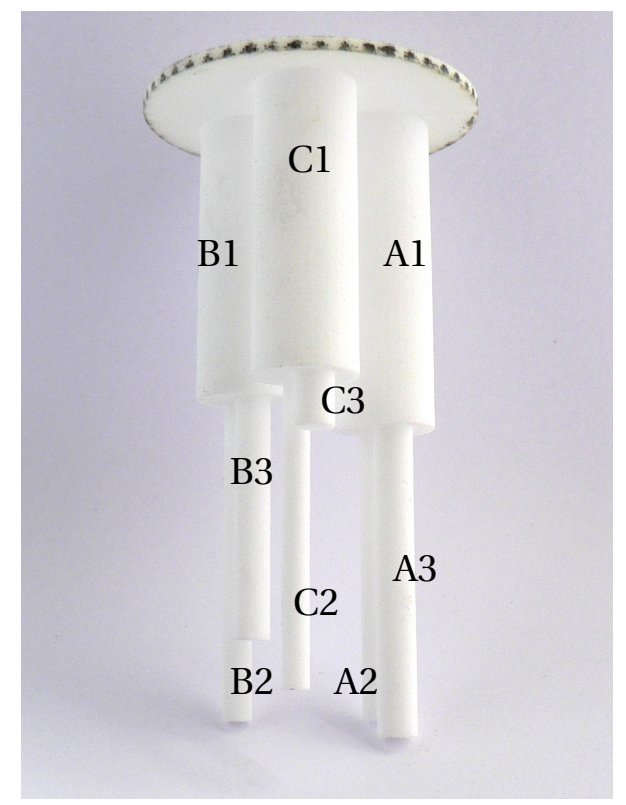

Figure B.2: Sample with 3 coupled tube resonators made with additive manufacturing technique.

\begin{tabular}{rrr|rrr|rrr}
$\mathrm{N}$ & $\begin{array}{r}L_{\text {design }} \\
{[\mathrm{mm}]}\end{array}$ & $\begin{array}{c}r_{t} \\
{[\mathrm{~mm}]}\end{array}$ & $\mathrm{N}$ & $\begin{array}{r}L_{\text {design }} \\
{[\mathrm{mm}]}\end{array}$ & $\begin{array}{c}r_{t} \\
{[\mathrm{~mm}]}\end{array}$ & $\mathrm{N}$ & $\begin{array}{r}L_{\text {design }} \\
{[\mathrm{mm}]}\end{array}$ & $\begin{array}{r}r_{t} \\
{[\mathrm{~mm}]}\end{array}$ \\
\hline $\mathrm{A} 1$ & 50.0 & 7.0 & $\mathrm{~B} 1$ & 45.0 & 7.0 & $\mathrm{C} 1$ & 45.0 & 7.0 \\
$\mathrm{~A} 2$ & 47.5 & 1.5 & $\mathrm{~B} 2$ & 52.5 & 1.25 & $\mathrm{C} 2$ & 47.5 & 1.0 \\
$\mathrm{~A} 3$ & 50.0 & 2.0 & $\mathrm{~B} 3$ & 40.0 & 1.75 & $\mathrm{C} 3$ & 10.0 & 2.0
\end{tabular}

Table B.2: Dimensions and coordinates of sample with 3 coupled tube resonators, according to design. Manufactured with a wall thickness of $1 \mathrm{~mm}$. 


\section{C}

\section{Test area for tyre/road contact: Twente Airport}

\section{Layout of test area}

The layout of the test area for tyre/road contact at Twente Airport is shown in Figure C.1. The reference road surface [2] (track 6) and the two prototype road surfaces (track 7 and 8) are indicated in light purple. The tracks were located at a service road on Twente Airport. All tracks were $3 \mathrm{~m}$ wide and $100 \mathrm{~m}$ long.

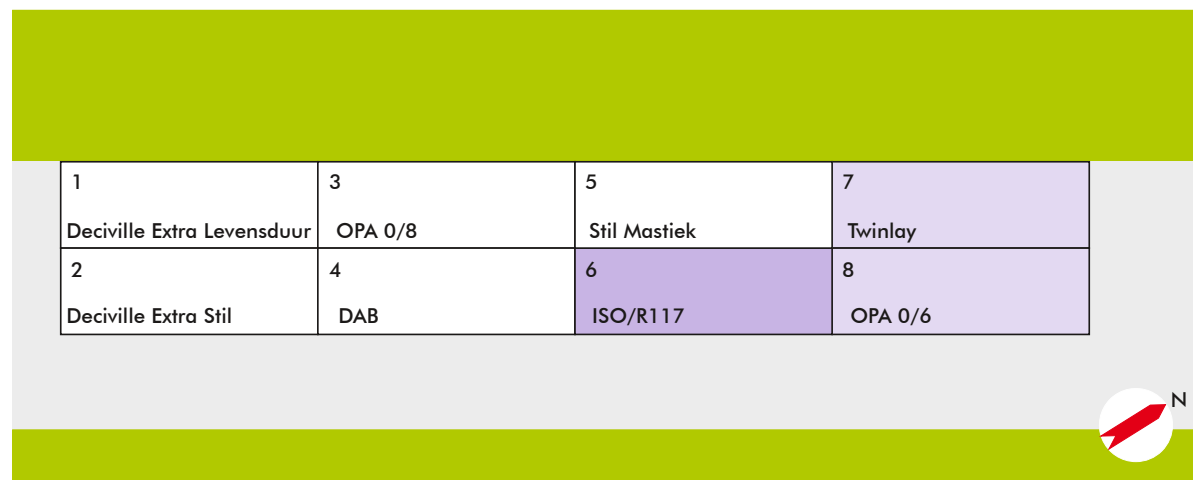

Figure C.1: Illustration of position of test tracks at test area.

\section{Properties of test tracks}

The properties of the 8 different test tracks are summarized below.

\section{Track 1: Deciville Extra Levensduur}

- Porosity: $12-13 \%$

- Stone size: $2 / 5 \mathrm{~mm}$

- Material stone: Bestone, $100 \%$ broken surface 
- Layer height: $25 \mathrm{~mm}$

\section{Track 2: Deciville Extra Stil}

- Porosity: $15 \%$

- Stone size: $2 / 5 \mathrm{~mm}$

- Material stone: Bestone, $100 \%$ broken surface

- Layer height: $30 \mathrm{~mm}$

\section{Track 3: OPA 8}

- Porosity: $20-21 \%$

- Stone size: $4 / 8 \mathrm{~mm}$

- Material stone: Bestone, $100 \%$ broken surface

- Layer height: $57 \mathrm{~mm}$

\section{Track 4: DAB}

- Porosity: $3-4 \%$

- Stone size: $4 / 8 \mathrm{~mm}, 8 / 11 \mathrm{~mm}, 11 / 16 \mathrm{~mm}$

- Material stone: Moräne, $>95 \%$ broken surface

- Layer height: $40 \mathrm{~mm}$

\section{Track 5: Stil Mastiek}

- Porosity: $6-10 \%$

- Stone size: $4 / 8 \mathrm{~mm}$

- Material stone: Graziet kleinhammer, 100\% broken surface

- Layer height: $35 \mathrm{~mm}$

Track 6: ISO/R117, [2]

- Porosity: $3-4 \%$

- Stone size: $2 / 6 \mathrm{~mm}, 4 / 8 \mathrm{~mm}$

- Material stone: Graziet kleinhammer, $100 \%$ broken surface

- Layer height: $35 \mathrm{~mm}$

\section{Track 7: Twinlay}

- Porosity (top layer): $25 \%$ 
- Stone size (top layer): $2 / 4 \mathrm{~mm}$

- Material stone (top layer): Porfier, SFB 3-100

- Layer height (top layer): $25 \mathrm{~mm}$

- Porosity (second layer): 25-26\%

- Stone size (second layer): 8/11

- Material stone (second layer):Moräne bitumen, 70/100

- Layer height (second layer): 50mm

\section{Track 8: OPA 6}

- Porosity: $25 \%$

- Stone size: $2 / 6 \mathrm{~mm}$

- Material stone: Bestone, SFB 3-100

- Layer height: $40 \mathrm{~mm}$ 



\section{Dankwoord}

Het zit erop. Na heel wat jaren sluit ik hiermee mijn periode op de Universiteit Twente af. Dit was een erg leuke en bijzondere periode waarbij ik heel veel aardige, behulpzame en gezellige mensen heb leren kennen. Ik ben hun hier dankbaar voor en wil bij deze de onderstaande personen in het bijzonder bedanken.

Allereerst wil ik André de Boer bedanken voor alle begeleiding, hulp en (praktische) inzichten die hij mij tijdens mijn promotie gegeven heeft. Maar ik wil hem ook zeker bedanken voor al zijn begrip en persoonlijke aandacht toen het met mijn gezondheid een stuk minder ging.

Daarnaast wil ik Ysbrand Wijnant bedanken voor zijn behulpzaamheid en (geduldige) begeleiding, waarbij hij mij zelf de richting in mijn onderzoek hebt laten bepalen. Ik heb erg veel gehad aan zijn veelzijde kennis en heb genoten van zijn enthousiasme over van alles en nog wat. Ik ben hem ook erg dankbaar dat bij Ysbrand mijn gezondheid altijd voor mijn onderzoek kwam.

Natuurlijk kan ik onze vakgroepmama, Debbie Vrieze-Zimmerman van Woesik, niet overslaan. Met haar persoonlijke aandacht voor iedereen en haar uitgebreide netwerk binnen de universiteit is Debbie onmisbaar voor onze groep. Debbie heeft altijd tijd voor een gezellig praatje en samen met het open deur beleid van André maken zij de sfeer binnen de groep fantastisch! Ook is Debbie de reden dat we met alle dames binnen onze groep nu eens in de zoveel tijd gezellig bij elkaar komen onder het genot van (zelfgemaakte) hapjes.

Ook onze man voor de technische ondersteuning, Axel Lok, wil ik bedanken. Zonder zijn hulp en tijd had ik lang niet zoveel en zulke goede metingen kunnen uitvoeren. Ook wil ik Axel bedanken voor alle keren dat hij samen met mij op het vliegveld heeft gestaan op vreselijk koude dagen in november of juist in de volle zon in juli om metingen te doen. Dankzij alle hulp en gezelligheid ging de tijd stukken sneller.

Ik wil ook mijn oud-kamergenoten Didem, Erwin, Jurnan, Jen-Hsuan, Dirk, Olti en Arthur bedanken voor hun gezelschap, interessante gesprekken en hulp. Ook de rest van onze groep kan ik niet vergeten en ik wil iedereen bedanken voor hun gezelligheid. En natuurlijk wil ik ook Belinda bedanken voor haar hulp en ondersteuning.

Buiten onze groep wil ik de werkplaats en vooral Theo Punt bedanken voor alle mooie samples die hij voor mij heeft gemaakt. Ook ben ik Gerben en Douwe van RaM dankbaar voor hun hulp die zij gegeven hebben om de rechtgeleider aan de praat te krijgen.

Dit onderzoek valt binnen het project Stil Veilig Wegverkeer en bij deze wil ik de mensen binnen dit project bedanken voor hun bijdrage aan mijn onderzoek. Met name Evert en Gerard van Reef en Dirk, Thijs, Bart en Bharat van Apollo wil ik bedanken. 
Natuurlijk wil ik bij deze mijn familie bedanken. Mijn ouders omdat zij mij altijd gestimuleerd hebben om te doen wat ik leuk vind en omdat zij mij altijd mijn eigen keuzes hebben laten maken. Ik ben hun dankbaar ook voor alle liefde en hulp die zij mij altijd hebben gegeven. Niet alleen tijdens het opgroeien, maar ook de afgelopen jaren toen mijn gezondheid keer op keer weer tegenviel. En dankzij alle dagen dat mijn ouders op Thijs hebben gepast, heb ik mij kunnen richten op het afronden van mijn promotie.

Tenslotte wil ik mijn lieve man bedanken. Maarten geeft mij rust en kan mij altijd opvrolijken, niet alleen door het delen van zijn inzichten en zijn liefde, maar ook door soms lekker gek te doen. Het is hartstikke fijn te weten dat Maarten mij altijd steunt en ook dat hij mij de tijd heeft gegeven om dit onderzoek af te ronden in mijn eigen tempo. Ik hoop dat wij met zijn tweeën nog lang samen zijn en dat er nog meer lieve kleine Thijsjes zullen volgen. 
UNIVERSITY OF TWENTE. 